Portland State University

PDXScholar

$1-1-1987$

\title{
The Stochastic Behavior of Soil Moisture and Its Role in Catchment Response Models
}

Nangantani Davies Godfrey Mtundu

Portland State University

Follow this and additional works at: https://pdxscholar.library.pdx.edu/open_access_etds Let us know how access to this document benefits you.

\section{Recommended Citation}

Mtundu, Nangantani Davies Godfrey, "The Stochastic Behavior of Soil Moisture and Its Role in Catchment Response Models" (1987). Dissertations and Theses. Paper 527.

https://doi.org/10.15760/etd.527

This Dissertation is brought to you for free and open access. It has been accepted for inclusion in Dissertations and Theses by an authorized administrator of PDXScholar. Please contact us if we can make this document more accessible: pdxscholar@pdx.edu. 


\title{
THE STOCHASTIC BEHAVIOR OF SOIL MOISTURE AND ITS ROLE IN CATCHMENT RESPONSE MODELS
}

by

NANGANTANI DAVIES GODFREY MTUNDU

A dissertation submitted in partial fulfillment of the requirements for the degree of

\author{
DOCTOR OF PHILOSOPHY \\ in \\ SYSTEMS SCIENCE/CIVIL ENGINEERING
}

Portland State University

1987 
TO THE OFFICE OF GRADUATE STUDIES AND RESEARCH:

The members of the Committee approve the dissertation of

Nangantani Davies Godfrey Mtundu presented June 1, 1987.

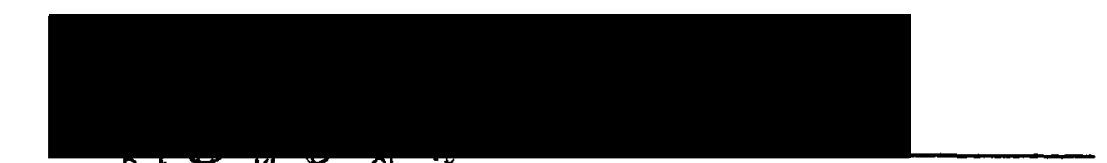

Roy \%. Kock, Chairman

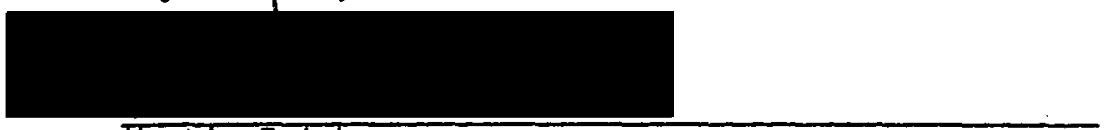

Mant in Zwick

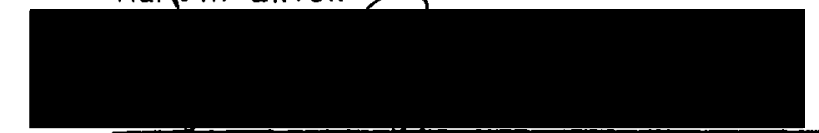

Vernon BisselT

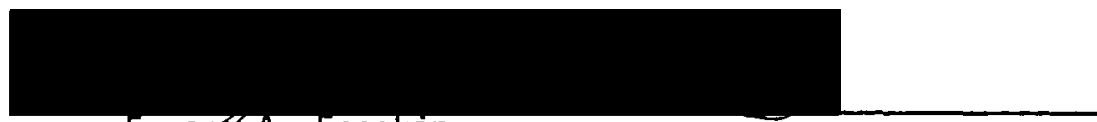

Eugege A. Enneking

APPROVED:

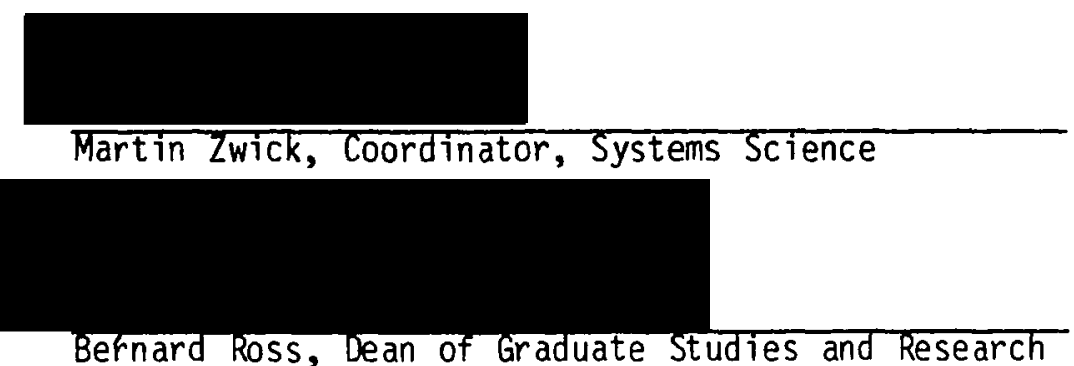


AN ABSTRACT OF THE DISSERTATION OF Nangantani Davies Godfrey Mtundu for the Doctor of Philosophy in Systems Science/Civil Engineering presented June 1, 1987.

Title: The Stochastic Behavior of Soil Moisiure and its Role in Catchment Response Models.

APPROVED BY THE MEMBERS OF THE DISSERTATION COMMITTEE:
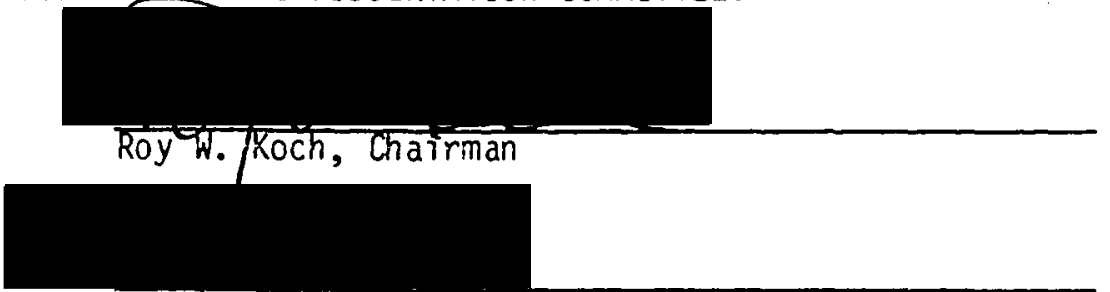

Martin Zwick

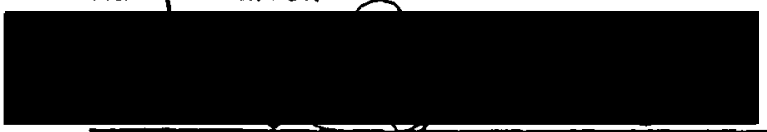

Vernon Bissel

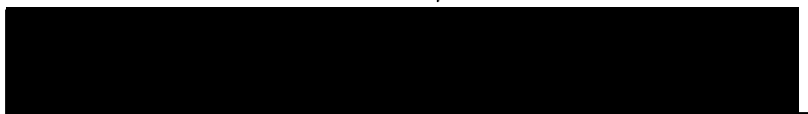

Eugene A. Enneking

Current efforts aimed at investigating models of catchment response have as their purpose the derivation of a physically based stochastic model of the watershed or catchment system in a manner which reflects the stochastic nature of the parameters of the physical system and the randomness of the climatic input processes. Recent studies have, however, indicated that a potentially limiting factor in deriving physically based models is the dependence of hydrologic response on 
initial soil moisture conditions. The dependence affects the distributions and moments of the hydrological processes being investigated. Therefore, there is a need to investigate the stochastic nature of soil moisture and, in particular, the soil moisture at the beginning of a storm event.

A stochastic model of soil moisture dynamics is developed in the form of a pair of stochastic differential equations (SDE's) of the Ito type. The sources of stochasticity in moisture dynamics are linked to the stochastic driving inputs of infiltration (directly related to a Poisson-process description of rainfall events) and the energy-related evapotranspiration (ET). One of the SDE's describes the "surplus" case, in which sufficient infiltration always occurs to allow for moisture depletion by the processes of drainage through and ET out of the root zone. The other SDE represents the "deficit" case, in which lack of adequate moisture leads only to an ET-controlled depletion process. The nature of the SDE's are such that they lead to sample functions involving Ito integral terms which can only be computed by numerical integration. However, analytical expressions are obtained for the moments, in particular the mean, variance and covariance. The analytical nature of the moment equations explicitiy shows the interrelationship between the stochastic properties of soil moisture, namely the mean, variance and covariance, and the parameters of the physical and climatic systems.

A quantitative evaluation of the stochastic model has been undertaken in order to assess the potential for application as a predictive tool and to identify major limitations. The basis of this 
Qvaluation is a comparison of the results outained from a Monte Carlo simulation using a simplified dynamic soil moisture model with those derived analytically from the stochastic model.

From the general model of soil moisture, a specific model of initial soil moisture, that is, the moisture at the beginning of a rain storm event, is developed and its moments are derived. Furthermore, the probability distribution of initial soil moisture is postulated to permit the assessment of how initial catchment "wetness" conditions affect the estimation of hydrologic response.

The moisture dymamics model reveals that the stochastic properties of soil moisture are sensitive to initial conditions in the watershed only for less permeable soils, such as clay loam, under the "surplus" state but are practically insensitive to such conditions for more permeable soils such as loam and sandy loam. The stochastic properties are, also, less sensitive to initial conditions for all soil types whenever under the "deficit" state. These results suggest that hydrologic processes, such as precipitation excess, infiltration, recharge and, hence, streamflow, will exhibit some dependence on initial soil moisture only in regions where the soils are, generally, less permeable and the $\mathrm{climate}$ is, for the most part, such that it sustains a "wet" environment. On the other hand, in arid or semi-arid regions, in which more permeable soils also tend to prevail, such hydrologic processes would exhibit no dependence on initial soil moisture conditions. These conclusions further imply that, in arid and semi-arid regions, an effective value of initial soil moisture, such as the mean, can be used to accurately estimate the properties of the hydrologic 
processes, whereas, in "wet" environments, more accurate values of the properties can only be obtained as weighted estimates based on the probability distribution of initial soil moisture. 


\section{ACKNOWLEOGEMENTS}

During the period of study and research leading to this dissertation, I have received far more than the usual share of time, effort and understanding from many people for all of which I am profoundly grateful. It would be a great pleasure to acknowledge all the contributions individually; however, the list would be endless and, consequently, I am only able to single out a smaller number.

I would particularly like to acknowledge my great indebtedness to my principal thesis advisor, Dr. Roy Koch, whose unwavering support during the whole period of study was directly responsible for this ultimate result. $\mathrm{Dr}$. Koch provided the inspiration, guidance, invaluable insights and both professional and financial assistance without which this work would not have come about. In one way or another, Dr. Koch's work has formed the basis of almost everything reported here and I consider myself uniquely fortunate to have had the opportunity to work with as dedicated a teacher and researcher that he certainly is.

I would also like to extend my special gratitude to Dr. Martin Zwick, my second thesis advisor, whose zealous pursuit of excellence was certainly a great inspiration and whose financial support during the latter part of the period of study could not have been offered at a better time. Many helpful comments and suggestions for improvement were also offered by Dr. Vernon Bissell, Dr. Eugene Enneking, and 
Dr. Herman Migliore for which I an very appreciative. I would also like to express my sincere gratitude to Dr. Franz Rad for providing administrative support and for taking much of the responsibilty for sustenance of financial support.

Much of the competence in dealing with the often "unfriendly" operating systems of the mini-computer that was used in much of the work was gained from to the never ending tutelage of Mr. Jon Griffin, and I would like to thank him for that. For her assistance on many an occasion and for her assistance with the final draft, Ms. Sally Lopez certainly deserves special thanks. I would also like to thank Ms. Vivian Gutierrez for typing most of the material in Chapters V, VI and VII and Mrs. Rebecca Bergeron for typing most of Chapter VIII. Last but certainly not least, I would like to express my utmost gratitude to $\mathrm{Mr}$. Eric Aroner for taking his valuable time to review the entire thesis and offering many valuable comments.

Finally, I would like to remind all other individuals who contributed either professionally or personally that their contributions are gratefully acknowledged and that it is only due to the limitations of space that they can not all be listed.

Thank you all! 
ACKNOWLEDGEMENTS $\ldots \ldots \ldots \ldots \ldots \ldots \ldots \ldots \ldots \ldots \ldots \ldots \ldots \ldots \ldots \ldots \ldots \ldots \ldots \ldots \ldots$

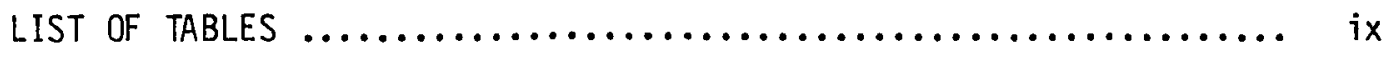

LIST OF FIGURES $\ldots \ldots \ldots \ldots \ldots \ldots \ldots \ldots \ldots \ldots \ldots \ldots \ldots \ldots \ldots \ldots \ldots \ldots \ldots \ldots$

CHAPTER

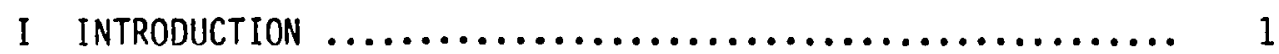

The Position of the Catchment System in the

Hydrological Cycle....................... 4

Research Objectives and Scope $\ldots \ldots \ldots \ldots \ldots \ldots \ldots . . . . . .9$

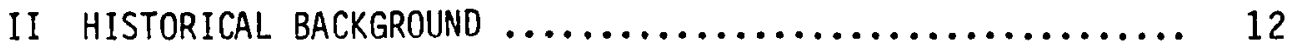

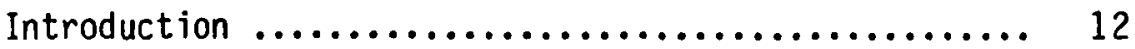

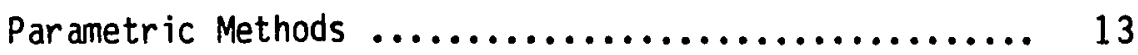

Stochastic Methods ......................... 14

Physically Based Stochastic Models .............. 16

Review of Progress $\ldots \ldots \ldots \ldots \ldots \ldots \ldots \ldots \ldots \ldots \ldots$

III THEORETICAL AND EMPIRICAL FRAMEWORK $\ldots \ldots \ldots \ldots \ldots \ldots \ldots . \ldots . \ldots 22$

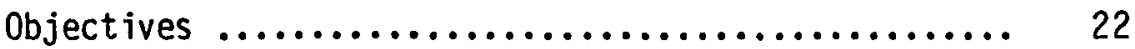

The Subsurface Flow Regime $\ldots \ldots \ldots \ldots \ldots \ldots \ldots \ldots . . \ldots 24$

The Horizontal Flow Regimes $\ldots . \ldots \ldots \ldots \ldots \ldots . . . . .27$

Modeling the Soil Water System ................ 27

IV MEAN SQUARE CALCULUS AND STOCHASTIC DIFFERENTIAL EQUATIONS 35

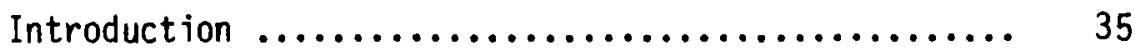


The General Stochastic Differential Equation ....... 37

Mean Square Calculus $\ldots \ldots \ldots \ldots \ldots \ldots \ldots \ldots \ldots . . \ldots \ldots$

Independent Increment Processes $\ldots \ldots \ldots \ldots \ldots \ldots \ldots .40$

The Wiener Process

The Compound Poisson Process

Stochastic Integration $\ldots \ldots \ldots \ldots \ldots \ldots \ldots \ldots \ldots . \ldots \ldots$

Numerical Methods for Stochastic Differential

Equations ............................ 48

V A STOCHASTIC DESCRIPTION OF SOIL MOISTURE ............ 51

A Simple Dynamic Soil Moisture Model ............ 51

The "Complete" Soil Moisture Simulation Model

Evaluation of the Simulation Model

Formulation of a Stochastic Differential Equation

Mode1 .............................. 62

VI SAMPLE FUNCTIONS AND MOMENTS OF SOIL MOISTURE ......... 72

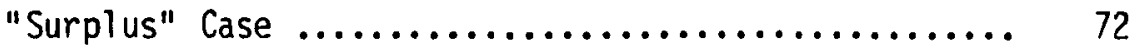

"Deficit" Case .......................... 75

Illustration of Solution Behavior ............. 76

"Surplus" Case

"Deficit" Case

Moment Properties $\ldots \ldots \ldots \ldots \ldots \ldots \ldots \ldots \ldots \ldots \ldots \ldots, 87$

"Surplus" Case

"Deficit" Case

VII EVALUATION OF THE STOCHASTIC MODEL OF SOIL MOISTURE ..... 97

Introduction $\ldots \ldots \ldots \ldots \ldots \ldots \ldots \ldots \ldots \ldots \ldots . \ldots \ldots$

Major Assumptions $\ldots \ldots \ldots \ldots \ldots \ldots \ldots \ldots \ldots \ldots . \ldots . \ldots$

Behavior of the Infiltration Process ........... 101

Occurrence of Limiting Behavior .............. 106 
Sensitivity to Initial Soil Moisture .......... 107

Effects of Other Simplifying Assumptions ......... 113

VIII THE STOCHASTIC BEHAVIOR OF INITIAL SOIL MOISTURE ....... 126

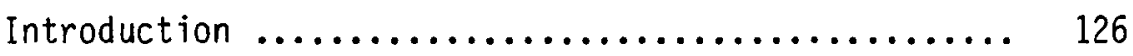

Moment Properties of Initial Soil Moisture......... 131

"Deficit" Case

"Surplus" Case

Distribution of Initial Soil Moisture $\ldots \ldots \ldots \ldots . . .135$

The Beta Distribution

Estimation of the Beta Distribution Parameters

Verification of the Beta Distribution Via Monte

Carlo Simulations and Goodness-of-Fit Tests

Comparison of Physically Based Beta Distribution

Parameters with Moment Estimators

IX APPLICATION OF THE INITIAL SOIL MOISTURE

PROBABILITY DENSITY FUNCTION ................. 160

Introduction $\ldots \ldots \ldots \ldots \ldots \ldots \ldots \ldots \ldots \ldots \ldots . \ldots \ldots$

Moments of Cumulative Infiltration ............. 161

Moments of Precipitation Excess ............... 162

Potential Effect of the Assumption of Constant-

Intensity Rain Storms ................... 166

$X$ RELATION OF SOIL MOISTURE DYNAMICS TO THE OVERALL

CATCHMENT RESPONSE: A DISCUSSION ............. 168

Introduction $\ldots \ldots \ldots \ldots \ldots \ldots \ldots \ldots \ldots \ldots . \ldots \ldots . \ldots \ldots$

The Deterministic Continuous-Time Reservoir Model ... 169

Summary of the Deterministic Reservoir Model ...... 175

The Stochastic Continuous-Time Reservoir Model ..... 176

The Outputs of the Stochastic Catchment Response

Model ................................ 179

XI SUMMARY, CONCLUSIONS, AND RECOMMENDATIONS .......... 181 
$\begin{array}{ll}\text { CHAPTER PAGE } & \text { PAT }\end{array}$

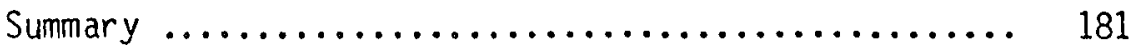

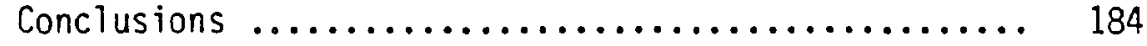

Stochastic Model of Soil Moisture Dynamics

Use of the Stochastic Model to Estimate Hydrologic Response

Recommendations for Further Research ........... 193

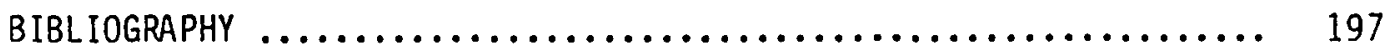




\section{LIST OF TABLES}

TABLE

PAGE

I Mean Intensity $(1 / \beta)$, Mean Duration $(1 / \lambda)$, Mean Interstorm Periods $(1 / \nu)$ of Rainfall Events and Mean Potential Evapotranspiration Rates for Two Selected $\mathrm{Cl}$ imates ...

II Typical Values Representing Subsurface Zone

Characteristics $\ldots \ldots \ldots \ldots \ldots \ldots \ldots \ldots \ldots \ldots \ldots \ldots$

III Comparison of the Results from the Simulation of the

Physical Soil Moisture Dynamic Model with Those from

the Stochastic Soil Moisture Model .............. 115

IV Kolmogorov-Smirnov Test Results for the Blacksburg, Va.

Climate: "Surplus" Case ....................... 150

$\checkmark$ Kolmogorov-Smirnov Test Results for the Denver, Co.

Climate: "Surplus" Case..$\ldots \ldots \ldots \ldots \ldots \ldots \ldots \ldots$

VI Kolmogorov-Smirnov Test Results for the Denver, Co.

Climate: "Deficit" Case ....................... 152

VII Chi-Square Test Results for Initial Soil Moisture ....... 154

VIII Comparison of Physically Based Beta Distribution

Parameters with Those Obtained on the Basis of

Monte Carlo Simulations of the "Complete" Moisture

Model (Koch and Mtundu, 1986a) ................... 155

IX Comparison of Physically Based Beta Distribution Parameters

with Those Obtained on the Basis of Monte Carlo

Simulations of the "Instantaneous Wetting and 
and Redistribution" Model (Koch and Mtundu, 1986a) ... 157

$X$ Comparison of Physically Based Moments of Normalized

Initial Soil Moisture with Those Based on

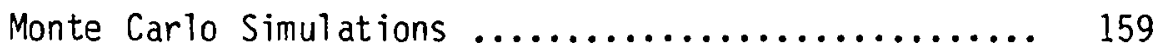

XI Moments of Cumulative Infiltration $\ldots \ldots \ldots \ldots \ldots \ldots \ldots . \ldots . \ldots 163$

XII Moments of Excess Precipitation ................... 165 
LIST OF FIGURES

FIGURE

PAGE

1. Schematic Outline of a Simplified River Basin System ..... 2

2. Block Diagram of the Hydrological Cycle .............. 5

3. The Catchment as a System ........................ 7

4. The Simplified Catchment System $\ldots \ldots \ldots \ldots \ldots \ldots \ldots \ldots . \ldots . \ldots$

5. Schematic Representation of an Idealized Catchment ...... 23

6. Schematic Representation of Soil Water Content Profile:

(a) Actual; (b) Sharp Wetting Front Approximation 30

7. Distinction Between the Ito and Stratonovich Evaluations

of the Stochastic Integral $\ldots \ldots \ldots \ldots \ldots \ldots \ldots \ldots .47$

8. A Simple Evapotranspiration Model ................ 55

9. The Drainage Function and its Linearized Approximation ... 57

10. Comparison of Simulated and Observed Sand Flume

Drainage Data $\ldots \ldots \ldots \ldots \ldots \ldots \ldots \ldots \ldots \ldots \ldots \ldots \ldots \ldots$

11. Simulation of Soil Moisture Variation in a Field of Strawberries (Tualatin Valley Irrigation District, Oregon). Crop Coefficient Varied During Season as Ind icated $\ldots \ldots \ldots \ldots \ldots \ldots \ldots \ldots \ldots \ldots \ldots \ldots \ldots$

12. A Section of the Sample Function of Soil Moisture

Evolution Process as Given by Eq. 6.9 with

$\beta^{2}=0.1 \mathrm{~cm}^{2} /$ day $\ldots \ldots \ldots \ldots \ldots \ldots \ldots \ldots \ldots \ldots \ldots \ldots \ldots \ldots \ldots \ldots \ldots \ldots \ldots$

13. An Amplified Portion of the Sample Function Given in

Fig. 12 
FIGURE

14. A Section of the Sample Function of Soil Moisture

Evolution Process as Given by Eq. 6.9 with

$\beta^{2}=1.0 \mathrm{~cm}^{2} /$ day $\ldots \ldots \ldots \ldots \ldots \ldots \ldots \ldots \ldots \ldots \ldots . \ldots \ldots \ldots$

15. A Section of the Sample Function of Soil Moisture Evolution

Process as Given by Eq. 6.13 with $\beta^{2}=1.0 \mathrm{~cm}^{2} /$ day .... 84

16. An Smplified Portion of the Sample Function Given in

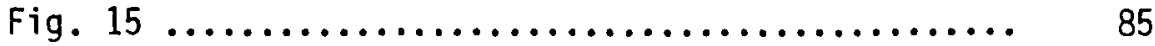

17. A Section of the Sample Function of Soil Moisture Evolution

Process as Given by Eq. 6.13 with $\beta=0.1 \mathrm{~cm} /$ day $\ldots . .86$

18. Mean of Cumulative Infiltration for Different Soil Types;

Blacksburg, Virginia Cl imate .................. 103

19. Second Moment of Cumulative Infiltration for Different Soil

Types; Blacksburg, Virginia Climate .............. 104

20. Mean Cumulative Infiltration Relative to Mean Storm Depth

for Different $\mathrm{Cl}$ imates; Clay Loam Soil ............ 105

21. Expected Value of Soil Water Content for Different Soil

Types; Blacksburg, Virginia Climate ("Surplus" Case) 109

22. Standard Deviation of Soil Water Content for Different Soil

Types; Bl acksburg, Virginia Climate ("Surplus" Case) 110

23. Expected Value of Soil Water Content for Different Soil

Types; Denver, Colorado Climate ("Deficit" Case) ..... 111

24. Standard Deviation of Soil Water Content for Different Soil

Types; Denver, Colorado Climate ("Deficit" Case) ..... 112

25. Comparison of Mean Soil Moisture Among Different Soil

Types; Denver, Colorado Climate ("Surplus" Case) ..... 116 
26. Comparison of Soil Moisture Variability Among Different Soil

Types; Denver, Colorado Climate ("Surplus" Case) ..... 118

27. Comparison of Mean Soil Moisture Among Different Soil

Types; Denver, Colorado Climate ("Deficit" Case) ..... 120

28. Comparison of So il Moisture Variability Among

Different Soil Types; Denver, Colorado Climate ("Deficit" Case) ........................... 121

29. Comparison of Autocorrelation Functions Among the Soil

Moisture Dynamic and Stochastic Models for the

Blacksburg, Virginia Climate ("Surplus" Case);

Clay Loam Soil ............................ 122

30. Comparison of Autocorrelation Functions Among the Soil

Moisture Dynamic and Stochastic Models for the Denver,

Colorado Climate ("Surplus" Case); Clay Loam Soil .... 124

31. Comparison of Autocorrelation Functions Among the Soil

Moisture Dynamic and Stochastic Models for the Denver, Colorado Climate ("Deficit" Case); Clay Loam Soil .... 125

32. Soil Moisture Evolution as a Filtered Poisson Process .... 127

33. Comparison Between the Cumulative Relative Frequency (CRF)

and the Theoretical Cumulative Distribution Function (CDF) of Normalized Initial Soil Moisture for the

Blacksburg, Virginia Climate ("Surplus" Case); Clay

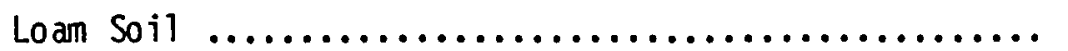


FIGURE PAGE

34. Comparison Between the Cumulative Relative Frequency (CRF) and the Theoretical Cumulative Distribution Function (CDF) of Normalized Initial Soil Moisture for the Blacksburg, Virginia $\mathrm{Cl}$ imate ("Surplus" Case); Loam Soil .................................. 142

35. Comparison Between the Cumulative Relative Frequency (CRF) and the Theoretical Cumulative Distribution Function (CDF) of Normalized Initial Soil Moisture for the Bl acksburg, Virginia Climate ("Surplus" Case); Sandy Loam Soil

36. Comparison Between the Cumulative Relative Frequency (CRF) and the Theoretical Cumulative Distribution Function (CDF) of Normalized Initial Soil Moisture for the Denver, Colorado Climate ("Surplus" Case);

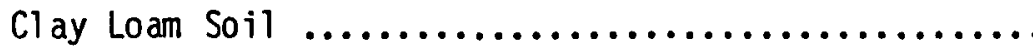

37. Comparison Between the Cumulative Relative Frequency (CRF) and the Theoretical Cumulative Distribution Function (CDF) of Normalized Initial Soil Moisture for the Denver, Colorado Climate ("Surplus" Case); Loam Soil.. 145

38. Comparison Between the Cumulative Relative Frequency (CRF) and the Theoretical Cumulative Distribution Function (CDF) of Normalized Initial Soil Moisture for the Denver, Colorado Climate ("Surplus" Case);

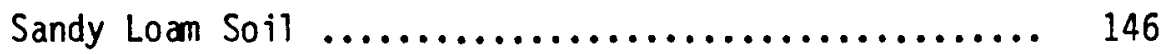


39. Comparison Between the Cumulative Relative Frequency (CRF) and the Theoretical Cumulative Distribution Function (CDF) of Normalized Initial Soil Moisture for the Denver, Colorado Climate ("Deficit" Case);

Clay Loam Soil $\ldots \ldots \ldots \ldots \ldots \ldots \ldots \ldots \ldots \ldots \ldots \ldots$

40. Comparison Between the Cumulative Relative Frequency (CRF) and the Theoretical Cumulative Distribution Function (CDF) of Normalized Initial Soil Moisture for the Denver, Colorado Climate ("Deficit" Case);

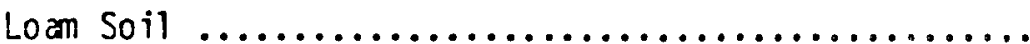

41. Comparison Between the Cumulative Relative Frequency (CRF) and the Theoretical Cumulative Distribution Function (CDF) of Normalized Initial Soil Moisture for the Denver, Colorado Climate ("Deficit" Case); Sandy Loam Soil 149

42. The Linear Reservoir Cascade; (a) Linear Storage Reservoirs; (b) The Corresponding Hydrographs ...... 173

43. General Summary of Model Development and Application ..... 185

44. Synthesis of the Stochastic Model of Soil Moisture Dynamics (Chapter V) $\ldots \ldots \ldots \ldots \ldots \ldots \ldots \ldots \ldots \ldots \ldots$

45. Analysis of the Stochastic Model of So il Moisture (Chapters VI and VIII) $\ldots \ldots \ldots \ldots \ldots \ldots \ldots \ldots \ldots \ldots \ldots$

46. Statistical Development of Probability Distribution of Initial Soil Moisture (Chapter VIII) $\ldots \ldots \ldots \ldots \ldots \ldots . . .188$ 47. Estimation of Moments of Hydrologic Processes (Chapter IX) 189 


\title{
CHAPTER I
}

\section{INTRODUCTION}

\begin{abstract}
Hydrology is the basic science underlying the design and management of water resources in that it provides the most basic information required in the social and economic development of the waters of the earth. This information is provided in the form of the quantity, quality, and spatial and temporal distributions of the water. Water resources are controlled and regulated to achieve a wide variety of conflicting objectives which can broadly be classified as quantity-control and quality-control. The former class may include municipal and industrial water supplies, hydroelectric power production, irrigation, navigation improvements and flood mitigation. The quality-control objectives might be appreciated in light of the reduction in utility of water for municipal, agricultural, and industrial uses that results from pollution, as well as in the diminution of the recreational and aesthetic value of lakes and rivers. Modern water resources projects are planned to achieve several of these objectives simultaneously, as illustrated in Fig. 1. Because of its multipurpose nature, the development of a water resources project is a complex task that usually involves a multiplicity of studies concerned with both the physical and socio-economic environment of man and, therefore, requires a long period for planning, design, construction and operation.
\end{abstract}




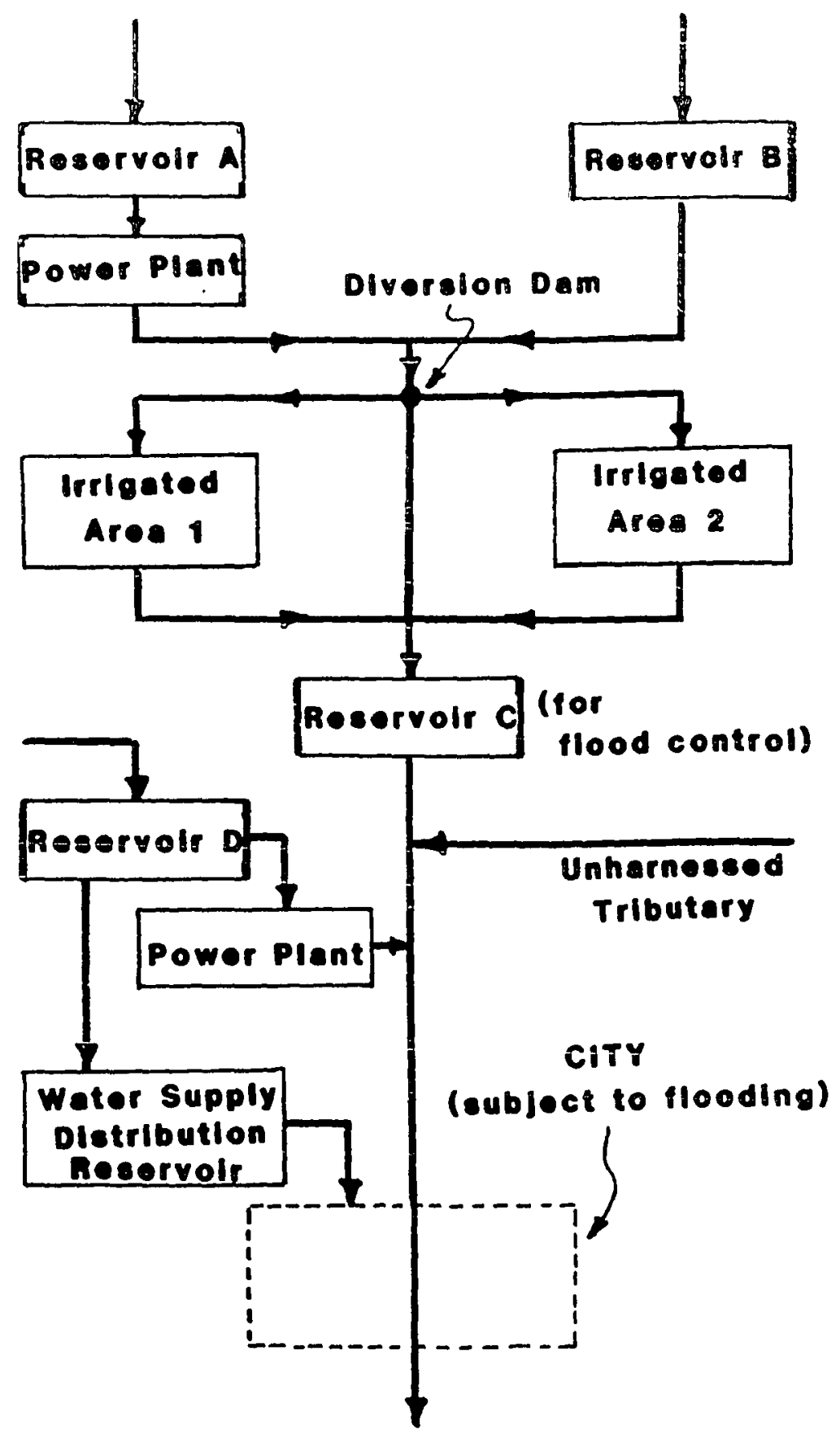

Figure 1. Schematic outline of a simplified river basin system. 
Planning for the unified development of a given water resources project consists of the collection of a data base followed by a series of decisions. The planning and operational stages are the ones in which hydrological information, both measured and estimated, is required.

One of the fundamental aspects in the practical application of hydrology is the estimation of basic elements of the hydrological regime. Such elements include the characteristics of streamflow, precipitation, evapotranspiration, dynamics of water masses, sediment transport and water quality, all of which are essential, directly or indirectly, for designing, constructing and operating water projects and hydraulic structures. The most relevant information for projects related to water quantity is, however, usually required in the form of possible future streamflow sequences. Unfortunately, reliable determination of streamflow estimates is complicated because of the need to account for the inherent uncertainty in the streamflow process itself. The streamflow process is an integral expression of sets of complex physiographic and climatic characteristics of the hydrological cycle, which are subject to random variations. The treatment of these random variations with respect to streamflow prediction forms the subject matter of this dissertation. In particular, the research leading to this thesis concentrated on aspects related to the problem of incorporating the stochastic behavior of the water existing in the unsaturated zone of the soil into existing models of overall catchment response to inputs of rainfall and evapotranspiration. 
THE POSITION OF THE CATCHMENT SYSTEM IN THE HYDROLOGICAL CYCLE

The basic elements of the hydrological cycle are best represented in the block diagram shown in Fig. 2 where the rectangles denote the various forms in which water is temporarily stored and the arrows denote the various hydrological processes responsible for the transfer of water from one form of storage to another. On a global scale, the hydrological cycle is a closed system in which the transfer processes are driven by the excess of incoming radiation $\left(R_{j}\right)$ and outgoing radiation $\left(R_{b}\right)$. The cycle may be viewed as a suprasystem consisting of such systems as the atmosphere, the drainage basins (catchment systems), the oceans and the 1 ithosphere (Dooge, 1973).

Any precipitable water $\left(W_{0}\right)$ in the atmosphere may be transformed by precipitation (P) to water stored in the catchment. This water is apportioned by the infiltration process (U) to the soil water system and overland flow $\left(Q_{0}\right)$. The infiltrated water replenishes the soil moisture storage from wich plants draw their water requirements, Upon satisfaction of this soil moisture deficit, there is either recharge (R) to the groundwater system or interflow $\left(Q_{j}\right)$ directly into the channel network system or both. The groundwater system may lose some of the water permanently to the lithosphere, but much of the storage is depleted by baseflow $\left(Q_{b}\right)$ to the channel network. Baseflow supplies most of the streamflow during dry periods. The overland flow, the lateral inflow, and the baseflow are all combined and routed in the channel network to form the runoff (R.O.) from the catchment system. Major losses from the catchment consist of either direct evaporation (E) 


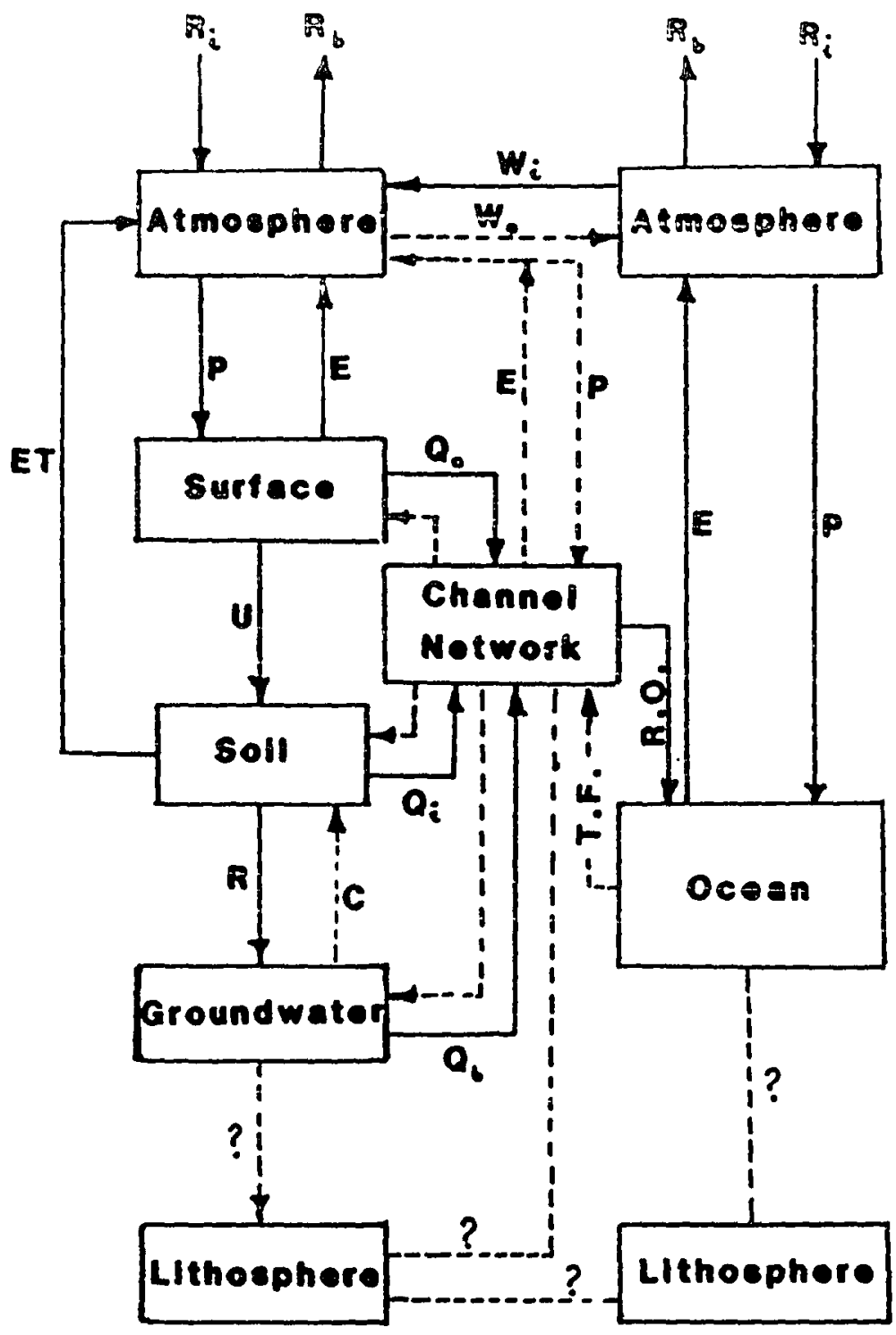

for legend, see Fig. 3 .

Figure 2. Block diagram of the hydrological cycle. 
from the surface regime or evapotranspiration (ET) from the unsaturated zone of the soil or both. These various hydrological processes form the subject matter of physical hydrology whose current rapid development, together with parametric and stochastic hydrologies, promises to play a very significant role in the methods of hydrologic system investigations.

In order to simplify analysis, objective methods of hydrologic system investigations do not involve the whole hydrological cycle as depicted in Fig. 2; rather, they are confined to one of the subsystems, namely the catchment, as shown in Fig. 3. Even this representation of the catchment is further simplified, for the practical purpose of runoff analysis, to that shown in Fig. 4. This simplified model, thus, divides the precipitation input $(P)$ into precipitation excess $\left(\mathrm{P}_{\mathrm{e}}\right)$ and infiltration $(U)$. The precipitation excess produces direct storm runoff $\left(Q_{s}\right)$, whereas the infiltration drives the processes of evapotranspiration (ET) and recharge (R). The recharge is routed through the groundwater system to emerge as baseflow $\left(Q_{b}\right)$ in the channel network. Finally, $Q_{s}$ and $Q_{b}$ are routed through the channel network to emerge as channel streamflow $\left(Q_{c}\right)$ at the outlet of the catchment.

The nature of the above hydrologic processes make the catchment a non-linear system. Both the surface processes that transform the precipitation excess into surface runoff and the subsurface processes that operate on the infiltrated water to ultimately produce baseflow are non-linear. Non-linearity in the subsurface processes is further enhanced by the presence of a threshold in the soil water storage 


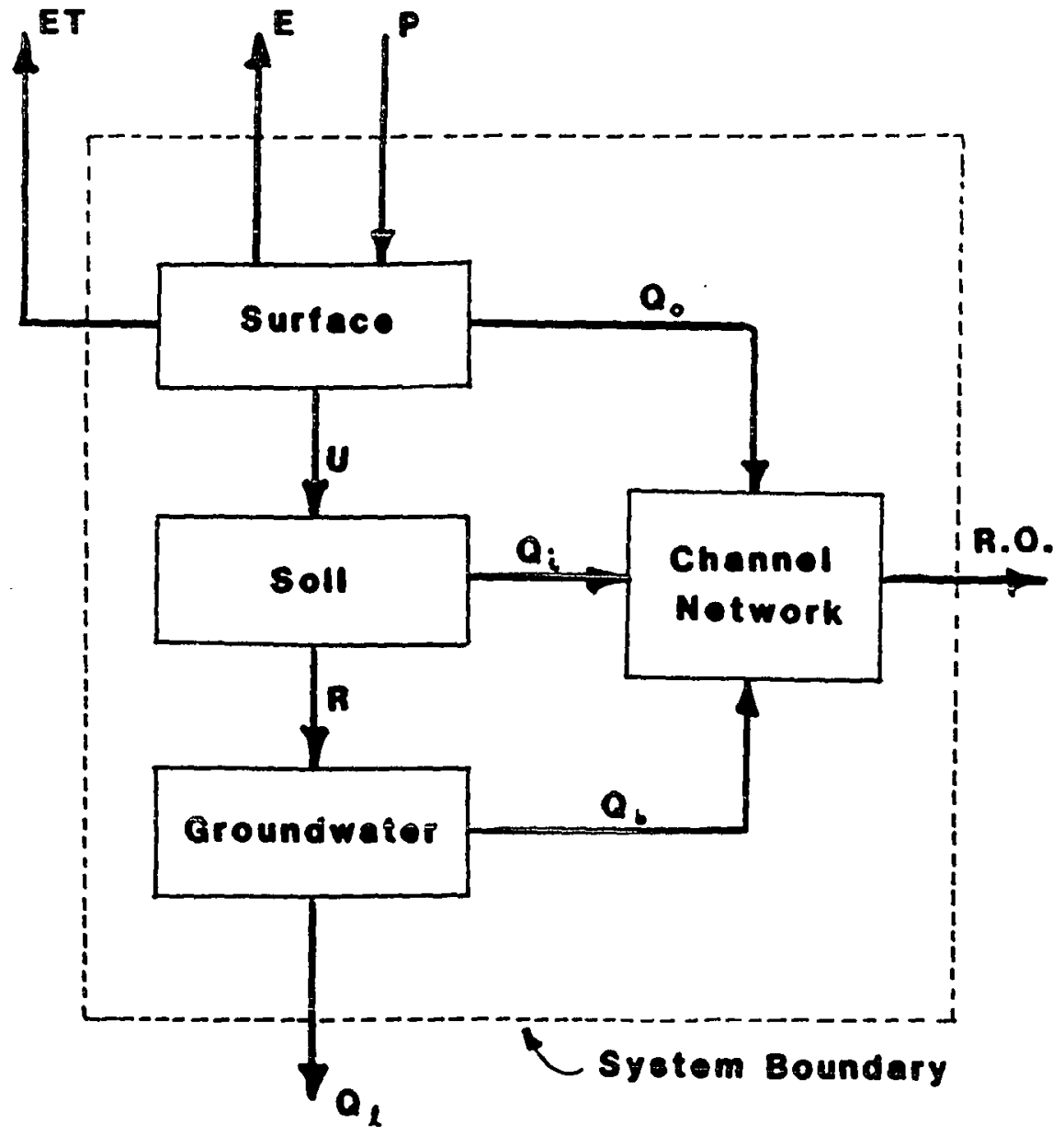

Legend: $\begin{array}{ll}P-\text { Precipitation } & a_{0}-\text { Overland flow } \\ T-\text { Transpiration } & a_{i}-\text { Interflow } \\ E-\text { Evaporation } & a_{b}-\text { Baseflow } \\ U-\text { Infiltration } & a_{i}-\text { Unaccounted flow } \\ \text { R }- \text { Recharge } & \text { R.0. - Runoff }\end{array}$

Figure 3. The catchment as a system. 


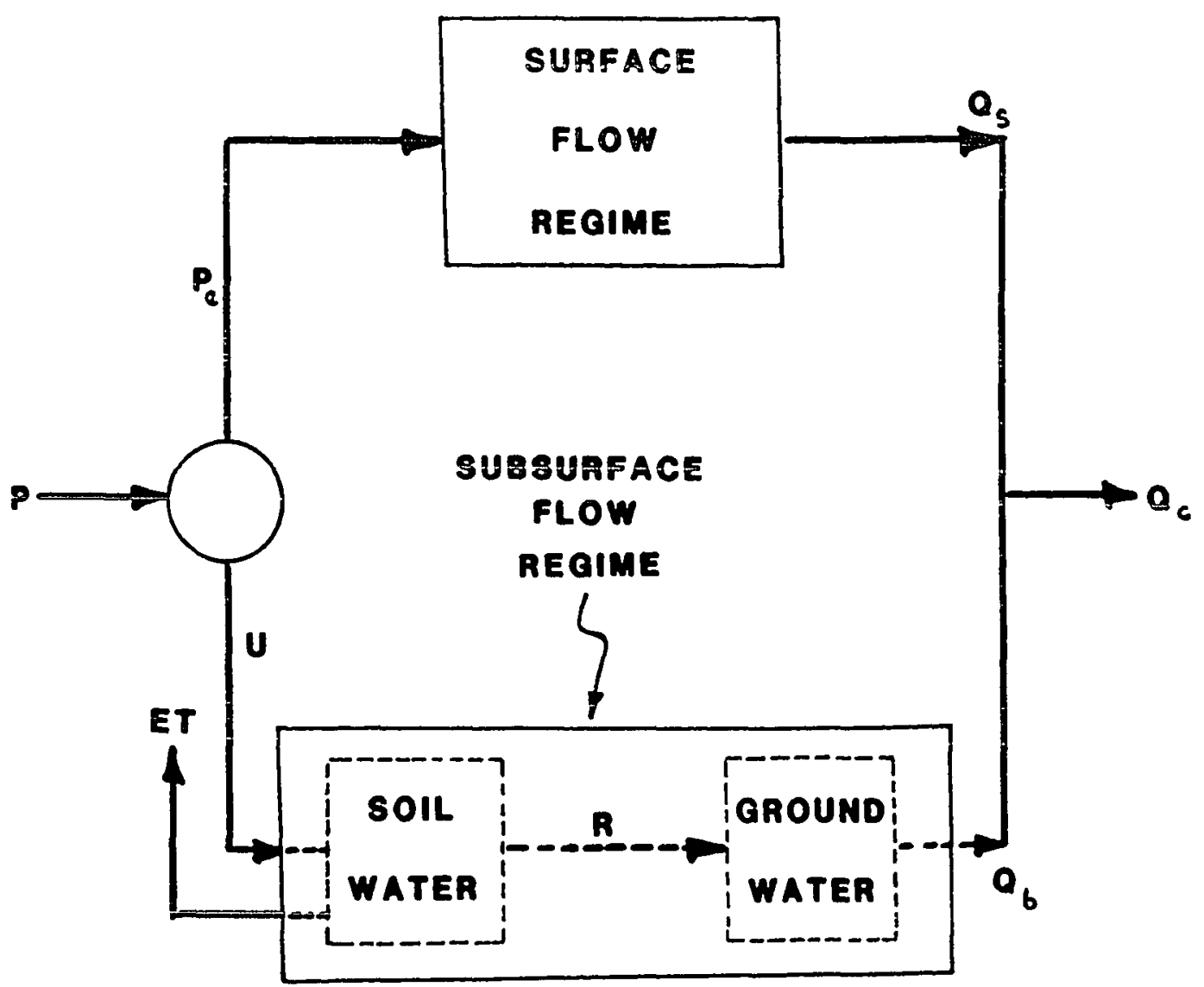

Figure 4. The simplified catchment system. 
phase. Despite the non-linearity which is inherent in the catchment system, significant insights into the behavior of the system have been gained by modeling certain of its components as if they were linear subsystems (Dooge, 1973). Nevertheless, past modeling approaches have had certain weaknesses which will be addressed in the present thesis. The major flaw in previous catchment response models has been in the assumption that the amount of water that infiltrates during any given precipitation event is some constant fraction of the incident rainfall. Such an assumption is obviously poor in 1 ight of the knowledge that has so far been gained in the physics of infiltration and soil water movement (e.g. Philip, 1969). Another aspect of the catchment which requires careful consideration is in the routing mechanisms in both the surface and subsurface flow systems. In particular, methods that attempt to determine the routing parameters without resorting to statistical manipulation of recorded data need to be improved. However, the scope in this dissertation will be limited to the stochastic behavior of soil moisture and the impact of this behavior on hydrologic processes, such as infiltration and surface runoff.

\section{RESEARCH OBJECTIVES AND SCOPE}

The principal objective of this research is to study how the stochastic behavior of the soil water system might be incorporated into existing stochastic models of catchment response to the inputs of rainfall and evapotranspiration.

The basic analysis involved in working towards the principal objective of this research includes the following procedural steps: 
1. Describe the basic inputs to the watershed (catchment), namely precipitation (rainfall) and evapotranspiration as stochastic processes; 2. Based on these stochastic inputs and the hydraulic properties of watershed soil, derive the stochastic properties of soil moisture, including sample functions, stochastic expectations (or moments) and a probability density function (POF);

3. Investigate the relationship between soil moisture in general and initial soil moisture, which is defined as the soil water content prior to a rainfall event;

4. On the basis of the PDF of initial soil moisture, "uncondition" the moments of those hydrologic processes that represent streamflow components which have been previously derived "conditionally" or by assuming some "fixed" value of initial soil moisture.

5. Evaluate the resulting models by using parameters of three types of soil in two study areas of widely different climatic characteristics.

The ultimate goal, the scope of which would exceed the requirements of a single dissertation, would be to derive a conceptual response model of the system in a manner which reflects the spatial variability of the hydraulic parameters of the soil and the randomness of the input processes. The use of such a model in the overall modeling of the catchment response would lead to a significant improvement in the estimation of the amount of water that runs off as part of rainfall excess and that which infiltrates into the soil. Given the amount of infiltration, the model would be used to track the movement of water through the processes of redistribution and drainage that ultimately lead to the recharge of the groundwater system. Such an 
approach would effectively eliminate the major flaw in previous models, namely the assumption that some constant "infiltration index" is responsible for apportioning the rainfall input between the surface flow and subsurface flow systems. Furthermore, prediction of the behavior of the soil water system would require that predictive relationships describing the hydraulic parameter distributions be incorporated into the soil dynamic response model. One would require that such parameter distributions be dependent on a priori estimation of the physical properties of the soil, such as texture, structure and particle size distribution, if the behavior prediction is to be carried out in the absence of measured hydraulic parameters. 
CHAPTER II

HISTORICAL BACKGROUND

\section{INTRODUCTION}

Traditionally, the study of the hydrological cycle has followed two major and seemingly independent directions. On the one hand, various research activities in the physical sciences have dealt with phenomena related directly or indirectly to the hydrological cycle without explicit recognition of their potential application in engineering design. On the other hand, hydrologic systems have been investigated for the more explicit purpose of establishing quantitative relationships between precipitation and runoff with the ultimate objectives of reconstructing historic sequences or predicting future ones. The former line of study is called "physical hydrology" whereas the latter may appropriately be termed "hydrologic system investigations" (Amorocho and Hart, 1964). The research described herein essentially falls under the latter category although it may be said to transcend the traditional disciplinary boundaries in that it requires the incurporation of physical hydrology concepts.

The methods of hydrologic system investigations have, in turn, historically been divided into two principal categories based on their approach. On the one hand, parametric (or deterministic) methods have sought to develop mathematical relationships among the various physical 
parameters involved in hydrologic events and to use these relationships to generate hydrologic (e.g. streamflow) sequences. On the other hand, stochastic methods have emphasized the use of the statistical characteristics of hydrologic variables such as streamflow, to generate stochastically similar occurrences of hydrologic sequences. Stochastic hydrology was developed essentially as a result of the recognition of the fact that future occurrences of hydrologic processes are uncertain and are, therefore, best described only in the probabilistic sense.

\section{PARAMETRIC METHODS}

The level of conceptualization in the deterministic methods of hydrologic system investigation has varied from a fairly sophisticated hydrodynamic level to a simple primitive level consisting of correlations between the hydrologic variables. Klemes (1978) and Amorocho and Hart (1964) give summaries of such methods. At the hydrodynamical level, the movement of water through the basin is governed by the St. Venant equations in the overland and channel flow regimes and by Darcy's law in the subsurface flow regime. The total outflow from the basin is then obtained by coupling these two flow regimes. An example of such a rigorously derived model is that presented by Freeze (1974), whereas Eagleson (1971, 1972) describes simplified forms of such a model.

At a slightly lower level of sophistication fall the systems-synthesis type of methods which involve the postulation of a somewhat less complex model consisting of a network of relatively few interacting hydrologic elements. The behavior of each element is 
described mathematically, and the whole model is calibrated by routing some recorded input and comparing the resulting output with recorded output. A typical model at this level is the Stanford Watershed Model originally proposed by Linsley and Crawford (1960). At the third level, one finds the unit hydrograph methods. The development of the concept of the unit hydrograph was one of the highlights of the golden age of classical hydrology (1930 to 1945) which concentrated on methods of predicting the runoff hydrograph. This hydrograph approach forms the basis of most conceptual lumped hydrological models which usually represent the basin as a single linear storage reservoir or as a cascade of such reservoirs (e.g. Nash, 1957). The concept has also been recently extended to a so-called geomorphologic unit hydrograph which relates the response of a basin to its geomorphologic structure (e.g. Rodriguez and Valdes, 1979). The unit hydrograph approach still remains as one of the most powerful tools of applied hydrology.

\section{STOCHASTIC METHODS}

Stochastic methods of hydrologic system investigations owe their development to the need for generation of so-called synthetic streamflow records for use in simulation studies of potential hydrologic designs. Early techniques (e.g. Rippl's mass curve analysis) used only the historical sequence of flows for a particular stream in their analyses. When hydrologists later realized that such sequences were neither likely to recur nor to include the worst flood or drought event, other techniques were sought, leading eventually to the development of linear stochastic hydrologic models. 
The basic idea behind this stochastic approach was to develop, by fitting procedures, models wich preserved the important statistical properties of one or more streamflow sequences such as trend, seasonality, mean, variance, skewness, serial correlation, crosscorrelation, and (rescaled) range. The resulting models would then be used to generate sets of equally likely occurrences of streamflow for use in an??ysis and design.

In general, the stochastic models were developed on the basis of linear Gaussian processes and without attempting to explain the underlying physical mechanisms. In addition, the models were mainly intended for the generation of monthiy and annual streamflow records.

DeCoursey (1982) classifies the discrete models as short-memory or long-memory models, the latter class being distinguished from the former in that they attempt to reproduce long-term characteristics. Short-memory models that have been proposed in recent times include moving average (MA) and autoregressive (AR) processes (e.g. Thomas and Fiering, 1962; Yevjevich, 1963), mixed auto-regressive moving average (ARMA) processes (Hipel et al, 1977; Salas et al, 1980) and autoregressive integrated moving average (ARIMA) processes (Salas et al, 1980). Long-memory models, include the fractional Gaussian-noise models (Mandelbrot and Wallis, 1968), broken-line processes (Mejia et al, 1972), certain forms of ARMA and ARIMA models (0'Connel1, 1974, 1977), and shifting-level models (Boes and Salas, 1978). Each of the above models is sequential and may be either univariate or multivariate. A non-sequential model which may have either short or long memory and be either univariate or multivariate is the disaggregation model proposed 
by Valencia and Schaake (1973).

The major weakness of all such stochastic models is their dependency on recorded hydrologic data with which to "fit" and test the models. This weakness is easily appreciated in light of the great lack of currently available data, on the one hand, and in light of the even grimmer prospect that the foreseeable future will still not provide enough data to accurately test these models, on the other hand.

\section{PHYSICALLY BASED STOCHASTIC MODELS}

Early studies in physically based stochastic models were merely attempts at justification of the application of the linear stochastic models such as the AR, MA, and ARMA processes. The studies were carried out on the basis of simple conceptualizations of the hydrologic processes with the aim of deducing the stochastic structure of monthly and annual streamflows. For instance, Yevjevich (1963) showed that annual streamflow could be modeled by a first-order Markov process. Salas and Smith (1981) demonstrated that annual streamflow sequences could be represented by ARMA processes through a mass balance description of the hydrologic system proposed by Fiering (1967). Quimpo (1973) showed that the unit hydrograph model leads to an autoregressive time series, thus establishing the relation between Markovian models and unit hydrograph parameters. Following Quimpo's lead, 0'Connor (1976) then demonstrated the correspondence between the various discrete linear conceptual hydrologic models and the linear transfer function models of Box and Jenkins (1976). Taking a different approach, and based on the observation that streamflow records are characterized by rapid rises and 
slow recessions, Weiss (1973) proposed the use of shot noise models to represent daily flow as a stochastic process. The shot noise model is a continuous-time model belonging to the class of models referred to as filtered Poisson processes described by Parzen (1962). It is, perhaps, a more realistic model than any of the others in its assumption that a streamflow model should be developed on the basis of the Poisson process rather than the Gaussian process.

A somewhat more physical approach to stochastic modeling was that undertaken by Eagleson $(1971,1972)$ who used a simplified form of the more rigorous hydrodymamical catchment model (Freeze, 1974) to analytically derive the probability distribution of peak streamflows. Input into the catchment model was represented by a simple joint distribution of areally averaged rainfall excess intensity and duration, the transformation from areal average rainfall being accomplished by a constant infiltration "loss" function. The kinematic wave was used to route the surface flow processes whose output was then combined with an empirically estimated constant ayerage yalue of baseflow in order to arrive at the total peak discharge. Building on Eagleson's approach, Wood (1976) introduced uncertainty into Eagleson's "loss" function by assuming, in turn, a uniform, an exponential and a gamma distribution, resulting in some improvement of the original model. Eagleson $(1978 a, b, c, d, e, f, g)$ later extended the approach to other hydrologic processes such as annual precipitation, infiltration, annual evapotranspiration and annual water yield. Freeze (1980), limiting his analysis to the hillslope scale and using Monte Carlo simulation techniques, investigated the influence of spatial stochastic structures 
of the hillslope parameters on the stochastic properties of the resulting runoff events and found this influence to be quite significant. More recently, Koch $(1982,1985)$ derived the distribution functions and stochastic properties of excess precipitation, infiltration and groundwater recharge for a single storm using simple probability distributions of precipitation characteristics and simple analytical expressions for the processes of infiltration, evapotranspiration, recharge and transfer mechanisms in a two-component model. The influence of initial soil moisture was, in this case, found to be significant. This finding is further investigated in the present study.

\section{REVIEW OF PROGRESS}

The above discussion of the methods of hydrologic system investigations has distinguished a number of levels of conceptualization in hydrologic modeling. The methods range in sophistication from the primitive level of empirical models to the deep hydrodynamical level of the classical mechanistic models. Implicit in the development of these methods has been the idea of conceptualizing a model which not only provides hydrological data sequences that facilitate design analysis, but also serves to explain the underlying physical processes, hence providing a link between physical hydrology and the parametric and stochastic hydrologies.

Despite their complexity, such physically based models as those presented by Eagleson (1978a,b,c,d,e,f,g) and Koch (1982, 1985) provide this important linkage as they serve to demonstrate the dependence of 
the stochastic properties of streamflow on the climatic and physiographic characteristics of the hydrologic regime. Furthermore, they allow for at least a qualitative evaluation of the effect of basin and climatic variables on the streamflow properties.

There is, however, a significant factor, one that may limit the predictive capability of the physically based models such as those cited above. The factor relates to the dependence of hydrologic response, primarily rainfall excess and recharge from storm events, on the initial soil moisture conditions at the beginning of astorm. This dependence affects the distributions and moments of the hydrologic processes. Eagleson $(1978 a, b, c, d, e, f, g)$ recognized the dymamic behavior of soil moisture but derived the annual water balance distribution and moments on the basis of an effective value of soil moisture that represented a space-time average.

The sensitivity to soil moisture at the beginning of the storm event has been corroborated in various other studies as well. For example, Diaz-Granados et al (1984), in deriving the distribution of flood peaks, demonstrated a marked sensitivity of the distribution function to soil moisture. In the stochastic models of cumulative infiltration, excess precipitation, recharge and streamflow derived by Koch (1982, 1985), the initial soil moisture was treated as a parameter, and a strong dependence between the initial conditions and the moments of streamflow was demonstrated. Using a slightly different rainfall model than Eagleson (1978b), Cordova and Rodriquez-Iturbe (1985) presented the mean and variance of surface runoff, as well as the probability of no runoff from a storm event, as a function of the 
initial soil water content.

All these efforts at physically based stochastic modeling have clearly been conditioned on the initial soil moisture. Therefore, the unconditional distributions (or moments) of the hydrologic processes can be obtained on the basis of the POF of initial soil moisture as:

$$
f(Q)=\int_{\theta} f\left(Q \theta_{i}\right) g\left(\theta_{i}\right) d \theta_{i}
$$

where

Q represents the hydrologic process;

$\theta_{j}$ is initial soil moisture;

$f(Q)$ is the unconditional function (pdf or expectation) of the hydrologic process $Q$;

$f\left(Q \mid \theta_{i}\right)$ is the conditional function of the hydrologic process given initial soil moisture; and

$g\left(\theta_{j}\right)$ is the pdf of initial soil moisture.

In addition to its importance in runoff prediction, there are other situations where the time evolution of soil moisture is required. For agricultural water management and irrigation scheduling in particular, soil moisture is the primary variable of interest.

Other than the work by Eagleson (1982) seeking to establish a space-time averaged value of soil moisture using ecological optimality arguments, there has been only limited work to uncover the stochastic nature of soil moisture. Cordova and Bras (1981), in order to establish optimal irrigation scheduling, developed a stochastic model of soil 
moisture based on Markov chains. Even though the transition probabilities of the Markov chains were computed via a physically based simulation model, the resulting distribution and moments were not presented in a manner that allows for the study of the interaction of climate, soil and vegetation systems in an explicit manner.

Given the importance of soil moisture in establishing initial conditions for hydrologic response, a study of the behavior of soil water content seems appropriate and, therefore, forms the principal objective in this dissertation. 


\section{CHAPTER III}

THEORETICAL AND EMPIRICAL FRAMEWORK

\section{OBJECTIVES}

This dissertation involves a study of the stochastic behavior of soil moisture which might be incorporated in a stochastic conceptual response model. The latter model may be used to generate streamflow sequences for any catchment given a few physically significant parameters. The parameters must be correlated with both the catchment characteristics and the stochastic properties of the inputs. The principal inputs are rainfall and evapotranspiration and the principal output consists of streamflow.

The development of such a model would, generally, be founded on the conceptualization of the catchment transfer mechanism as a linear storage reservoir consisting of two major components: the surface flow regime and the subsurface flow regime, as illustrated in Fig. 4 (Chapter I) or, schematically, in Fig. 5. The emphasis in this research is on the important vertical linkage between these flow regimes which are essentially horizontal systems. This vertical linkage is represented by the processes that take place in the unsaturated soil zone. The importance of this linkage is derived from the fact that the soil processes determine the amount and time distribution of precipitation excess that is available for surface storage and runoff and, hence, the 


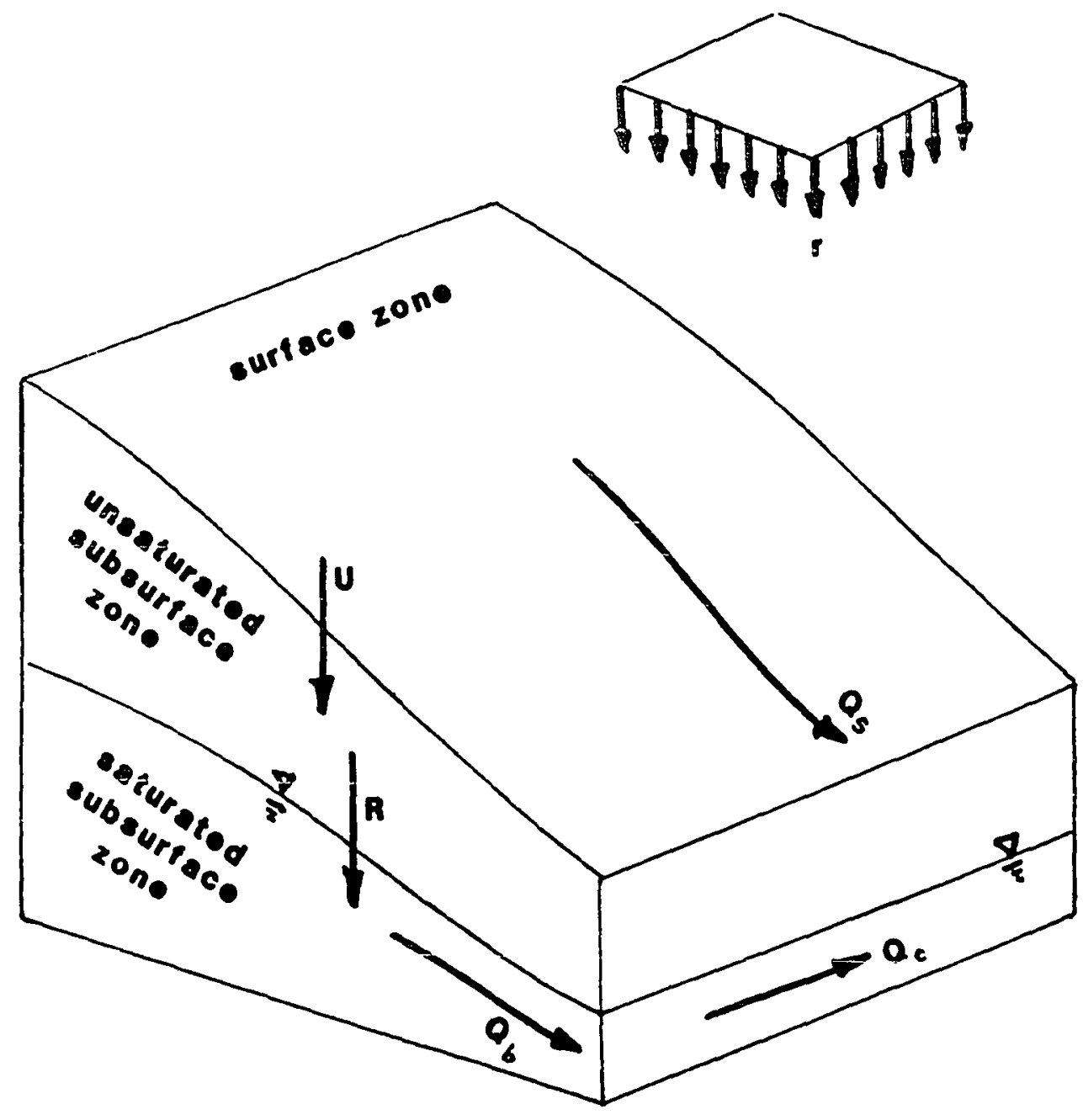

Figure 5. Schematic representation of an idealized catchment. 
amount and time distribution of infiltrated water that is available for subsurface storage, evapotranspiration, and baseflow. Consideration of this phase of the catchment has, for the most part, involved rather gross simplificatons such as the use of a constant lumped "loss" function to represent the amount of infiltration (e.g. Eagleson, 1972; Bodo and Unny, 1985). A rigorous hydrodynamic description of this important vertical linkage may be summarized as follows.

THE SUBSURFACE FLOW REGIME

The subsurface flow processes linking the surface flow system and the groundwater system include infiltration, redistribution and recharge. In a hydrodynamical model, these processes involve partial differential equations whose solutions are subject to appropriate boundary and initial conditions.

The basic equations governing subsurface flow processes in the soil water system are derived by combining Darcy's law for threedimensional flow in homogeneous isotropic media and the continuity equation. The two equations are written, respectively, as:

$$
\begin{gathered}
\mathbf{q}=-K(\theta) \nabla \Psi \\
\partial(\rho \theta) / \partial t=-\nabla \cdot \rho \mathbf{q}
\end{gathered}
$$

where $\rho$ is the density of water, $\theta$ is the volumetric soil water content, $K(\theta)$ is the moisture-dependent hydraulic conductivity of the soil, $\Psi$ is the total potential, $\mathbf{q}$ is the moisture flux vector, $t$ is the time and 
$\boldsymbol{\nabla}$ is the vector differential operator. The resulting form of the general equation for flow in the soil zone then becomes:

$$
\partial(\rho \theta) / \partial t=\nabla \cdot[\rho K(\theta) \nabla \Psi]
$$

The main components of the total potential are the capillary (hydrostatic pressure) potential and the gravitational potential. When expressed in terms of energy per unit weight, the total potential may be written as:

$$
\Psi=\psi(\theta)+Z
$$

in which $\psi(\theta)$ is the capillary potential and $Z$ is the gravitational potential.

Considering a one-dimensional system, in which flow takes place only in the vertical Z-direction (oriented downwards), and assuming $\rho$ is constant (which it is for most practical purposes), and using Eq. 3.4, Eq. 3.3 is reduced to the so-called Richards' equation:

$$
\partial \theta / \partial t=\partial / \partial Z[D(\theta) \partial \theta / \partial Z]+\partial K(\theta) / \partial Z
$$

where $D(\theta)=K(\theta) d \psi / d \theta$ is the soil moisture diffusivity. Eq. 3.5 represents one form of the Richards' equation used for modeling flow in the unsaturated soil zone. The solution of this equation, be it analytical or numerical, requires some evaluation of the highly non-linear soil properties, $K(\theta)$ and $D(\theta)$, which are, therefore, usually 
given in the form of empirical soil moisture characteristic curves. In place of these curves, however, empirical relationships have been suggested by various investigators. For instance, Dagan and Bresler (1983) suggested the following set of constitutive relations:

$$
\begin{gathered}
K(\psi) / K_{s}=\left(\psi_{w} / \psi\right)^{n} \\
\theta^{*}=\left(\theta-\theta_{r}\right) /\left(\theta_{s}-\theta_{r}\right)=\left(\psi_{w} / \psi\right)^{\beta}
\end{gathered}
$$

where $K_{S}$ is the hydraulic conductivity at natural saturation, $\psi_{W}$ is the air-entry value ("bubbling" pressure), $\theta_{S}$ is the saturation water content, $\theta_{r}$ is the residual water content for which $K$ tends to zero, and $\eta$ and $\beta$ are empirical coefficients. From Eqs 3.6 and 3.7, one can deduce the following relationship:

$$
K(\theta)=K_{S}\left(\theta^{*}\right)^{n}
$$

where $n=n / \beta$. The form given by Eq. 3.8 was al so suggested by Corey (1977).

The Richards' equation (3.5) forms the basis for describing the processes of infiltration and soil moisture redistribution. The output from the latter process is the recharge to the groundwater system. 
THE HORIZONTAL FLOW REGIMES

The first horizontal flow system consists of surface flow phenomena, namely, the overland flow $\left(Q_{S}\right)$ and the stream channel flow $\left(Q_{C}\right)$. Such surface flow dynamics can be approximated by the one-dimensional kinematic wave model whose description consists of the continuity equation and a simplified representation of the dynamic equation in the form of a single-valued stage-discharge relation. The kinematic wave is, of course, an approximation of the complete dynamic wave (St. Venant equations) which is governed by the continuity equation, describing the conservation of mass, and the dynamic equation, which expresses the conservation of momentum.

The second horizontal flow regime consists of the groundwater flow systems occurring below the water table. A rigorous description of these flow phenomena is given by the Laplace equation for saturated flow. This equation is obtained by combining Darcy's law with the continuity equation under the assumptions that the soil medium is homogeneous and isotropic and the water is incompressible.

\section{MODELING THE SOIL WATER SYSTEM}

Rather than use the rigorous characterization of the soil water dynamics through the Richards' equation (3.5), approximate models obtained by simplifying the Richards' equation (e.g. Dagan and Bresler, 1983), or by simple formulations based on Darcy's law (e.g. Mein and Larson, 1973; Morel-Seytoux and Khanji, 1974), or by derivations based on mass balance (e.g. Koch, 1982) are used in the development of the 
model described herein. On the one hand, the Darcy-type equations or the simplified forms of the Richards' equation are utilized to characterize the process of infiltration in terms of soil properties and boundary conditions. On the other hand, the mass balance-type of equations are used to describe the soil moisture redistribution process.

One approach to developing an approximate moisture-flow model involves solving the Richards' equation (3.5) subject to the following boundary and initial conditions:

$$
\begin{aligned}
& \theta=\theta_{i}, \quad t=0, \quad z \geq 0 \\
& q=q_{0}, \quad 0 \leq t \leq t_{f}, \quad z=0, \quad \theta(0, t)<\theta_{s} \\
& q=0, \quad t>t_{f}, \quad z=0
\end{aligned}
$$

where $\theta_{i} \geqslant \theta_{r}$ is an assumed uniform initial soil water content, $q_{0}$ is a given constant water flux at the soil surface, $t_{f}$ is the total infiltration time and the other variables are as defined previously. Eq. 3.10 is replaced by:

$$
\theta=\theta_{s}, \quad t_{p} \leq t \leq t_{f}, \quad z=0
$$

if $\theta(0, t)$ reaches the saturated value, $\theta_{S}$, before infiltration ceases. Solving Eq. 3.5 under these conditions typically yields, for 
any time $t$, the soil water distribution shown in Fig. 6(a), where the wetting front (which is the limit of water penetration into the soil) shows a gradual decrease in soil water content with depth. However, a fairly accurate solution may also be obtained by assuming a sharply defined wetting front, as illustrated in Fig. 6(b). Under this assumption, Dagan and Bresler (1983) developed an approximate moisture flow model given by the following ODE:

$$
\mathrm{dU} / \mathrm{dt}=\mathrm{K}_{0}\left(\theta_{0}^{*}\right)+\left[\left(\psi_{\mathrm{w}} /(\eta-1)\right]\left[\mathrm{K}_{0}\left(\theta_{0}^{*}\right)^{-1 / \beta}-K_{i}\left(\theta_{i}^{*}\right)^{1-1 / \beta}\right] / z_{0}\right.
$$

where $Z_{0}=U /\left(\theta_{0}-\theta_{j}\right), K_{j}=q\left(Z_{f}, t\right)$ is the gravitational drainage at the wetting front (at depth $Z_{f}$ ), $U$ is the cumulative infiltration and $K_{0}$ and $\theta_{0}$ are the averaged $K$ and $\theta$ values over $0 \leqslant Z_{0} \leqslant Z_{f}$.

Eq, 3.13 is valid for any time, $t$, as long as $q_{0}<k_{s}$. For $q_{0} \geqslant K_{S}$, the equation is applicable only in the range $0<t<t_{p}$, where $t_{p}$ is the ponding time, defined as the time for which $\theta^{*}$ reaches unity and $K$ reaches $K_{S}$. The appropriate ODE, after certain simplifying assumptions, that is applicable for $t \geqslant t_{p}$ is:

$$
d U / d t=K_{S}+\left[K_{s} \psi_{w}\left(\theta_{s}-\theta_{j}\right)\right] /[(n-1) U]
$$

and $t_{p}$ is given by:

$$
t_{p}=\psi_{w}\left(\theta_{s}-\theta_{i}\right) k_{s} /\left[(n-1)\left(q_{0}-k_{s}\right) q_{0}\right]
$$




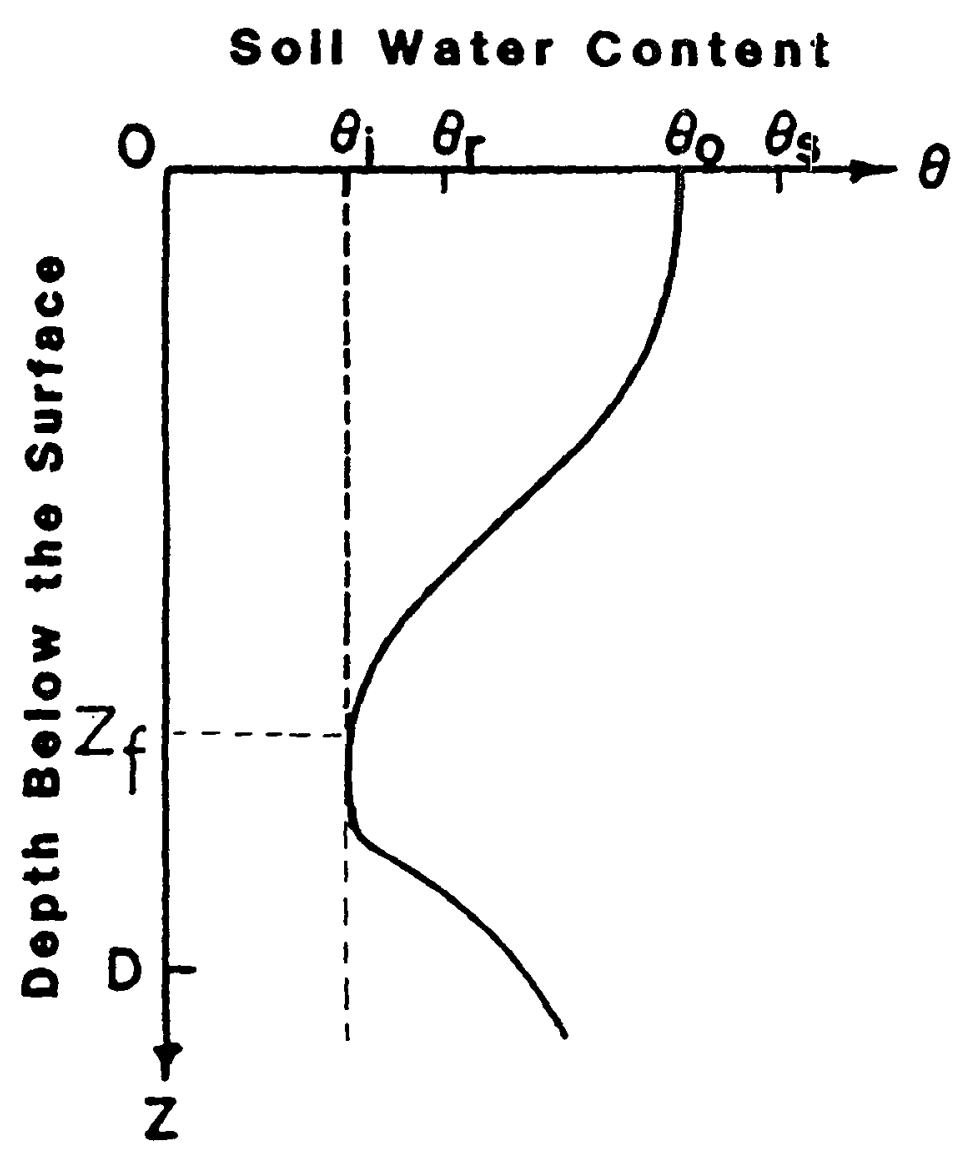

(a)

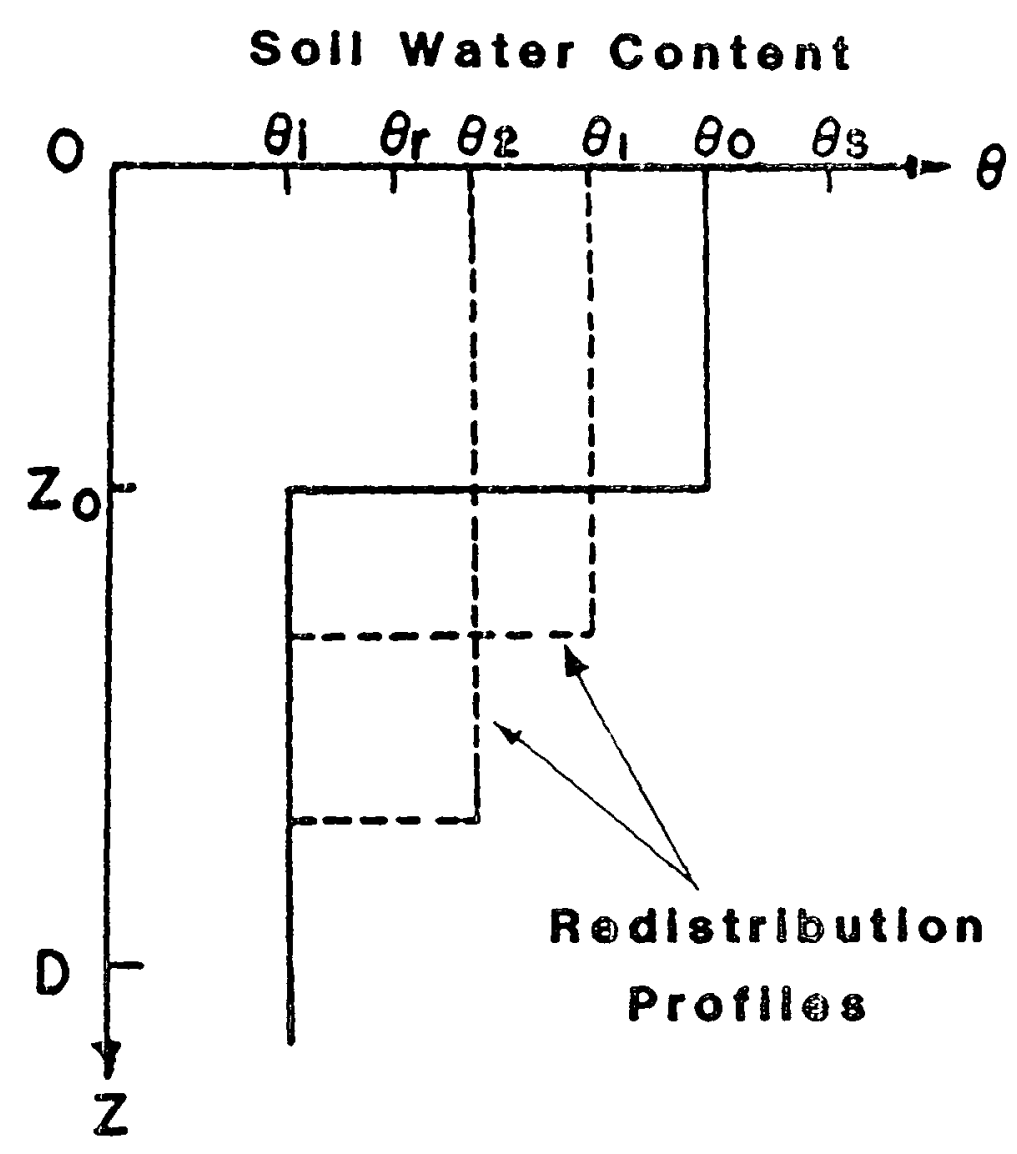

(b)

Figure 6. Schematic representation of soil water content profile:

(a) actual; (b) sharp wetting front approximation. 
Eqs 3.13 and $3.14 \mathrm{can}$, therefore, be used to determine the amount of infiltration into the unsaturated zone of the soil profile under constant flux conditions. Alternatively, the Mein and Larson (1973) approximate method may be used for this purpose. The Mein-Larson method represents a modification of a semi-empirical infiltration model developed by Green and Ampt (1911). The Green-Ampt model was developed by direct application of Darcy's law to the problem of infiltration from a ponded surface into a deep homogeneous soil with a uniform initial soil water content. The model may be represented by the equation:

$$
d U / d t=K_{s}\left(H_{0}+S_{f}+Z_{f}\right) / Z_{f}
$$

in which $\mathrm{H}_{0}$ is the depth of water ponded on the surface and $S_{f}$ is the effective suction at a sharply defined wetting front. Mein and Larson (1973) applied the Green-Ampt model under rainfall conditions by determining the cumulative infiltration $\left(U_{p}\right)$ at the time of surface ponding $\left(t_{p}\right)$ from Eq. 3.16 to obtain:

$$
d U / d t=\left\{\begin{array}{l}
r, \quad t<t_{p} \\
k_{s}+k_{s}\left(\theta_{s}-\theta_{i}\right) s / U, \quad t \geq t_{p}
\end{array}\right.
$$

where the ponding time is given by: 


$$
t_{p}=s^{2} /\left[2 r\left(r-k_{s}\right)\right]
$$

and where $r$ is a constant rainfall rate and $S$ is the soil sorptivity at the sharp wetting front. The sorptivity of the soil is a measure of the capillary flow characteristics of the initial soil moisture and is given by:

$$
S=\left[2 K_{s}\left(\theta_{s}-\theta_{i}\right) H_{c}\right]^{0.5}
$$

In this equation, $\mathrm{H}_{C}$ is the "capillary drive" which represents the effects of capillary forces on the unsaturated flow and is also a function of initial soil moisture.

For the purposes of this research, however, the infiltration model presented by Morel-Seytoux (1980), which is a modification of a relation proposed by Philip (1969), is used. The model, which takes into account the effects of air in the soil, is given by:

$$
d U / d t=\left\{\begin{array}{l}
r, \quad t<t_{p} \\
k_{s} / B+s /\left[2\left(t-t_{p}+t_{c}\right)^{0.5}\right], \quad t \geq t_{p}
\end{array}\right.
$$


accounting for air resistance in the soil and $t_{c}$ is a correction factor that accounts for ponding infiltration and depends on the ponding-time formula used. The factor is obtained such that at ponding time, the infiltration rate should equal the rainfall rate whereas the cumulative infiltration should equal the total rainfall depth, i.e. $U_{p}=r t_{p}$. Furthermore, the infiltration rate should approach $K_{S}$ as time increases. Based on these considerations and $\mathrm{Eq}$. 3.18, the correction factor is given by;

$$
t_{c}=s^{2} /\left[4\left(r-k_{s}\right)\right]^{2}
$$

All the various ways of describing the infiltration process discussed above (Eqs. 3.13 to 3.21 ) lead to practically the same behavior of soil moisture. Therefore, the choice of which model to use is based on convenience in analysis.

In order to describe the soil-moisture redistribution and drainage processes, another approach involving a simplified form of the Richards' equation can also be used. The approach utilizes a continuity equation derived on the basis of a direct mass balance of soil water, and is given by (Koch, 1982):

$$
Z_{f} d \theta / d t=f-q-E T
$$

where $f=d U / d t$ is the infiltration rate and $Z_{f}=U /\left(\theta-\theta_{j}\right)$ is the depth of the wetting front. Recharge to the groundwater system begins 
when the wetting front reaches the base of the unsaturated soil layer at depth D, approximately, and hence is a function of the redistribution process. The redistribution process is illustrated in Fig. $6(\mathrm{~b})$. Now that the framework for the theory and empiricism that forms the basis for this research has been established, the next thing to do is to focus on the stochastic behavior of soil moisture, in general, and the stochastic behavior of initial soil moisture, in particular, and how such behavior is related to the overall problem of predicting the response of catchments, such as surface runoff, to the climatic inputs of rainfall and evapotranspiration. However, before proceeding along this line of investigation, a review of stochastic differential equations (SDE's) and their associated stochastic calculus is presented. This important tool of SDE's is subsequently used to formulate and obtain solutions for the stochastic soil moisture dymamics model. 
CHAPTER IV

MEAN SOUARE CALCULUS AND STOCHASTIC DIFFERENTIAL EQUATIONS

\section{INTRODUCTION}

Stochastic differential equations arise when the elements which give rise to the representations of continuous dynamical systems as ordinary differential equations are considered subject to environmental fluctuations or noise. The expanding volume of literature pertaining to SDE's and their associated stochastic calculus attests to its well developed theory and its wide application in most branches of science and engineering. Unfortunately, most of the literature seems to place so much emphasis on rigor and completeness that it is useful to present a brief review of the subject. The scope is naturally limited to an explanation of the solutions to the type of equations formulated in the following chapter in connection with soil moisture dynamics. Furthermore, all the conditions under which the presented formulae and results are valid are not mentioned for the sake of brevity.

Inasmuch as the physical laws governing unsaturated flow are well known, the moisture process can be described in terms of a differential equation for the time rate of change of water content, $\theta(t)$. However, given the hydraulic properties of the soil, the time evolution of soil moisture is due principally to environmentally controlled sources of moisture, cumulative infiltration and energy, the last being 
reflected in the loss of moisture due to evapotranspiration, ET. As such, much of the forcing must be considered stochastic, leading to the description of soil water content as a stochastic differential equation (SDE). In spite of this realization, most physically based stochastic models, heretofore, have been developed using the derived distribution approach to determine either the distribution or moments of the output. This approach has severe limitations when dealing with variables that continuously evolve in time and are thus described as stochastic processes. This dissertation deals with a simplified differential equation for soil moisture, presenting the solution process and moments directly. This approach provides a generalized framework for the solution of many hydrologic problems where the basic process is described in terms of fluid mechanics or thermodynamics but with random environmental influences.

As indicated above, a differential equation, and thus its solution, becomes stochastic when it involves random elements. Following the categorization of Soong (1973), the sources of stochasticity can be traced to the initial conditions, the forcing function, the coefficients, or some combination of these factors. In the particular problem discussed here, the differential equations exhibit randomness in all of these elements.

Given the SDE, a number of properties of the solution can be determined. First, the most complete description is the stochastic process, or the transition probability density, which is obtained from the solution of the Fokker-Planck equation (e.g. Soong, 1973), such that the probability distribution of the solution is determined at all 
subsequent times. The Fokker-Planck equation is, however, generally very difficult to solve and may not be practically useful (e.g. Bodo et al, 1985). An alternative is the direct solution of the SDE to obtain the functional relationship between the solution variable and the stochastic components of the process. The result permits the determination of a sample function of the stochastic process which may be used in simulation. Finally, practical interest may rest with the first few moments of the solution process which may be obtained directly from the sample function or via the Ito differential rule (e.g. Soong, 1973).

As general as the SDE approach may appear, there are limitations on the types of processes which can be used to describe the random elements. In general, solutions are only known for independent increment processes. The most practically useful are the Brownian motion as a continuous process and the Poisson process as random point process (Gihman and Skorohod, 1972). However, the solutions are known for a fairly broad class of differential equations, including those involving vector-valued solution variables. A concise review of the stochastic calculus and solution procedures for SDE's is presented by Bodo et al (1985). Further, Bodo and Unny (1985) have demonstrated the application of these procedures to a conceptualized catchment model.

THE GENERAL STOCHASTIC DIFFERENTIAL EQUATION

The stochastic model of soil moisture to be developed subsequently takes the form of the following formal representation of the general stochastic differential equation: 
$d X_{i}=a(X, t) d t+b(X, t) d W_{i}+\int_{U} c(x, t, u) M(d t, d u)$

where $X_{t}$ is the stochastic process representing a solution of the SDE, $W_{t}$ and $M(d t, d u)$ are, respectively, the Wiener process and the compound Poisson process to be defined momentarily and a, b, c are coefficients linear in $X$ and $u$. This SDE should be understood as an abbreviation of the following integral equation:

$x_{t}=x_{0}+\int_{t_{0}}^{t} a(x, \tau) d \tau+\int_{t_{0}}^{t} b(x, \tau) d \psi+\int_{t_{0}}^{t} \int_{U} c(x, \tau, u) M(d \tau, d u)$

The reason for this interpretation is that stochastic calculus is essentially an integral calculus, with no independently defined derivative.

The solution process $x_{t}$ of the general SDE may be defined only insofar as the integrals appearing in Eq. 4.2 can be given some "meaning". Since integrals are generally defined in terms of "limiting sums", the concept of "limit" itself in stochastic calculus needs to be defined.

\section{MEAN SQUARE CALCULUS}

The relevant calculus that has evolved for SDE's is the calculus in mean square or m.s. calculus. Mean square calculus is rooted in the concept of m.s. or second-order convergence and is primarily concerned with linear operations on second-order random variables (r.v.'s) and stochastic processes. Second-order r.v.'s are real r.v.'s $X_{1}, X_{2}, \ldots$ 
whose second moments $E\left[X_{1}^{2}\right], E\left[X_{2}^{2}\right], \ldots$ are finite.

In order to extend the methods of analysis of deterministic functions to random functions, some space (called the Hilbert space) is defined in which linear operations on second-order $r . v . ' s$ are carried out. In deterministic calculus, functions are defined on the real line $R$. A little reflection shows that in limit processes, one uses the fact that, on the space $R$, there is available a distance function, $d$, which associates a distance:

$$
d(x, y)=|x-y|
$$

with every pair of points $x, y$ on $R$. The function $d(x, y)$ is, thus, the metric defined on space $R$.

In the Hilbert space, the metric is given by:

$$
d\left(x_{1}, x_{2}\right)=\left\{E\left[\left(x_{1}-x_{2}\right)^{2}\right]\right\}^{0.5}
$$

Furthermore, a norm and a scalar product are also appropriately defined. The appropriate mode of convergence for sequences of second-order r.v.'s is second-order or m.s. convergence defined in terms of the appropriate metric as follows: the sequence $\left\{x_{n}, n=1,2, \ldots\right\}$ of r.v.'s converges to a r.v. $X$ in m.s. if

$$
\lim _{n \rightarrow \infty} E\left[x-x_{n}\right]^{2}=0
$$

and if $E\left[X_{n}\right]$ is finite for all $n$. 
Once m.s. convergence is defined, m.s. continuity, m.s. differentiability, and m.s. integrability follow in the same manner as in the deterministic calculus.

\section{INDEPENDENT INCREMENT PROCESSES}

As indicated in the introduction to this chapter, solutions to SDE's are, generally, known for a special type of processes called the independent increment processes. The best known of these processes are the Wiener process and the Poisson process, both of which play a fundamental role as noise models in SDE-theory.

The Wiener Process

The Wiener process $\{W(t), t \geqslant 0\}$, al so called the Wiener-Levy process or the Brownian motion process, is defined by the following properties:

(i) $\quad W(0)=0$ with probability one (w.p. 1);

(ii) $E[W(t)]=0$ and $E[d W(t)]=0$;

(iii) $E\left[W^{2}(t)\right]=\sigma^{2} t$ and $E\left[\left(d W_{t}\right)^{2}\right]=\sigma^{2} d t$;

(iv) $E[W(t)-W(s)]^{2}=\sigma^{2}(t-s), t>s>0$;

(v) $\quad E[W(t) W(s)]=\sigma^{2} \min (t, s)$;

(vi) $\{W(t), t>$ 아 has independent increments;

where $d W(t)=W(t+d t)-W(t)$. In words, $W(t)$ is normally distributed with mean zero and variance $\sigma^{2} t, \sigma^{2}>0, t>0$, and written:

$$
W(t) \sim N\left(0, \sigma^{2} t\right)
$$


Frequently, the normalized Wiener process with $\sigma^{2}=1$ is used. For the normalized Wiener process, $\left\{W^{\prime}(t), t \geqslant 0\right\}$ :

$$
\begin{aligned}
& W^{\prime}(t)-N(0, t) \text {; and } \\
& {\left[W^{\prime}(t)-W^{\prime}(s)\right] \sim N[0,(t-s)], t>s>0 .}
\end{aligned}
$$

Other properties of the Wiener process are as follows:

(vii) $W(t)$ is continuous and also m.s. continuous almost everywhere but nowhere differentiable;

(viii) $W(t)$ is of unbounded variation, that is:

$$
\max _{n} \sum_{j=1}^{n}\left|w\left(t_{j+1}\right)-w\left(t_{j}\right)\right|
$$

is not finite for any sequence of partitions $s=t_{1}<t_{2}<\ldots<t_{n+1}=t$.

The Wiener process provides a fairly adequate model for the noise process called Gaussian white noise, $\{\xi(t), t \geqslant 0\}$. Symbolically, the modei is written:

$$
\xi(t)=\frac{d W(t)}{d t}
$$

This formal derivative must be interpreted as:

$$
W(t)=\int_{0}^{t} \xi(\tau) d \tau
$$

where $\xi(t)$ is a random function of zero mean and zero correlation at 
times $t \neq s$. The function $\xi(t)$ provides a useful approximation to the so-called wide-band noises, that is, noises with very small correlation times.

The Compound Poisson Process

The compound Poisson process is defined in terms of the simple integer-valued Poisson process $\{N(t), t \geqslant 0\}$ that counts the incidence of point events on $[0, \infty)$. The simple Poisson process may be specified by either the counting specification or the intensity specification. Using the former, the process is defined by the following properties:

(i) $\quad N(0)=0 \quad$ w.p. 1 ;

(ii) the increment $[N(t)-N(s)], 0 \leqslant s<t$, is Poisson-distributed with parameter $v$, i.e. the probability of $k$ events is given by:

$$
P\{N(t)-N(s)=k\}=\left[v(t-s)^{k}\right] \exp [-v(t-s)] / k !
$$

for $k=0,1,2, \ldots$

(iii) $\{N(t), t \geqslant 0\}$ has independent increments.

For a compound Poisson process, each incidence or occurrence point is associated with a non-zero variable or mark $u$, drawn independently of the process $\{N(t), t \geqslant 0\}$ from the mark space $U$ according to the cumulative distribution function (CDF), $F_{U}(u)$, with mean

$E[U]=\lambda$. The compound Poisson process $X(t)$ can then be represented as: 


$$
x(t)=\int_{t_{0}}^{t} \int_{U} u M(d \tau, d u)
$$

where, given that $T \subset[0, \infty)$ and $B \subset U$, the quantity $M(T, B)$ represents the number of points occurring in volume TXB (see Snyder, 1975 or Bodo et a), 1985).

A wide variety of phenomena encountered in physics and engineering $c$ an be mathematically represented by the so-called Poisson-driven Markov process which satisfies an integral equation of the form:

$$
x(t)=x_{0}+\int_{t_{0}}^{t} a(x, \tau)+\int_{t_{0}}^{t} \int_{U} c(x, \tau, u) M(d \tau, d u)
$$

As developed in Snyder(1975), the last integral in (4.9) has the evaluation given by:

$$
\int_{t_{0}}^{t} \int_{U} c(x, \tau, u) M(d \tau, d u)= \begin{cases}0, N(t)=0 & (4.10) \\ \sum_{j=1}^{N(t)} c\left[x\left(\tau_{j}\right), \tau_{j}, u_{j}\right], N(t)>1 & \end{cases}
$$

where $N(t)$ is the number of incident points during $\left[t_{0}, t\right), \tau_{j}$ and $u_{j}$ are the time of occurrence and mark, respectively, of the $j$ th point. 


\section{STOCHASTIC INTEGRATION}

As indicated before, the solution of the general SOE (Eq. 4.1 or 4.2) has some "meaning" only insofar as the integrals appearing in Eq. 4.2 can be appropriately defined. Depending on the form of the integral, stochastic integration can be defined either in the Riemann sense or the Riemann-Stieltjes sense. Given the integral:

$$
Y(t)=\int_{t_{0}}^{t} f(\tau) d X(\tau)
$$

where $f(t)$ is a deterministic function and $x(t)$ is a second-order random function on $\left[t_{0}, t\right)$, one would use the Riemann-Stieltjes definition. This definition involves taking the partition $t_{0}=t_{1}<t_{2}<\ldots<t_{k+1}=t$ and forming the approximating sum:

$$
Y_{k}=\sum_{j=1}^{k} f\left(\tau_{j}\right)\left[x\left(t_{j+1}\right)-x\left(t_{j}\right)\right], \quad t_{j}<\tau_{j}<t_{j+1}
$$

If the m.s. limit or 1 imit in the mean $(1 . i, m$.$) given by:$

$$
\prod_{\substack{k \rightarrow \infty \\ \rho \rightarrow 0}} \underbrace{}_{k} \cdot Y_{k}=Y_{t}
$$

(where $\rho=\max _{j} \Delta t_{j}, \Delta t_{j}=t_{j+1}-t_{j}$ )

exists independently of the choice of $\tau_{j}$ and the partition, then the 
random function $Y_{t}$ is called the m.s. Riemann-Stieltjes integral of $f(t)$ with respect to $x(t)$ on $\left[t_{0}, t\right]$. However, if both $f(t)$ and $x(t)$ are random functions, defined on the same probability space, an attempt to define a unique stochastic integral in the above manner fails.

In Eq. 4.2, the first integral can be defined as a m.s. Riemann integral, but the second one cannot be defined as a Riemann-Stieltjes integral for the reason stated above. The value of the second integral depends on the choice of the location of $\tau_{j}$ at which the random function $b(X, \tau)$ is evaluated in the approximating sums on each interval $\left[t_{j}, t_{j+1}\right]$ of the partition. Consequently, Ito (1951), defined integrals of the form:

$$
Y_{t}=\int_{t_{0}}^{t} G(s) d W(s)
$$

for a broad class of so-called non-anticipating functionals $G(s)$ of the Wiener process $W(t)$. A function $G(s)$ which is independent of the increments $[W(t+s)-H(t)]$ for all $s>0$ is called a non-anticipating functional.

The so-called Ito integral is, thus, defined by specifying the integrand at the beginning of the interval; that is, by choosing $\tau_{j}$ $=t_{j}$. The integrand $G(s)$, therefore, becomes a non-anticipating functional of $W(t)$, being statistically dependent on $W(u)$ for $u<t$; that is, on the "past" only. The Ito interpretation leads to correct results only if the integrand can be considered to be independent of the Wiener increments.

By choosing $\tau_{j}=\Delta t_{j} / 2$, that is, by specifying the integrand 
at the mid-point of each interval $\left[t_{j}, t_{j+1}\right]$ of the partition, Stratonovich (1966) defined the so-called symmetricized stochastic integral. Thus the Stratonovich interpretation leads to correct results only if the integrand is regarded as being correlated to the Wiener increment.

The distinction between the Ito interpretation of the stochastic integral and that of Stratonovich is more clearly made with the aid of Fig. 7. In evaluating the stochastic integral as an Ito integral, the integrand $G(s)$ is evaluated at time $t_{k-1}$ for the partition $\left[t_{k-1}, t_{k}\right]$. Inasmuch as the integrand and the integrator [in this case, the Wiener increment $\mathrm{dW}(\mathrm{s})]$ are regarded as independent in such an evaluation, the expected value of the integral becomes zero, that is, because of independence:

$E\left[\int_{t_{0}}^{t} G(s) d W(s)\right]=\int_{t_{0}}^{t} E[G(s)] E[d W(s)]$

But since $E[d W(s)]=0$, by definition of the Hiener process, one gets:

$E\left[\int_{t_{0}}^{t} G(s) d W(s)\right]=0$

One can also, therefore, show (Hoel et al, 1972) that, for $a \leqslant b \leqslant c$ :

$$
E\left[\int_{a}^{b} f(t) d W(t) \int_{a}^{c} g(t) d W(t)\right]=\sigma^{2} \int_{a}^{b} f(t) g(t) d t
$$

where $\sigma^{2}$ is the variance of the Wiener process.

In the Stratonovich integral, the integrand is evalauted at the symmetric mid-point of the partition $\left[t_{k-1}, t_{k}\right]$, resulting in the 


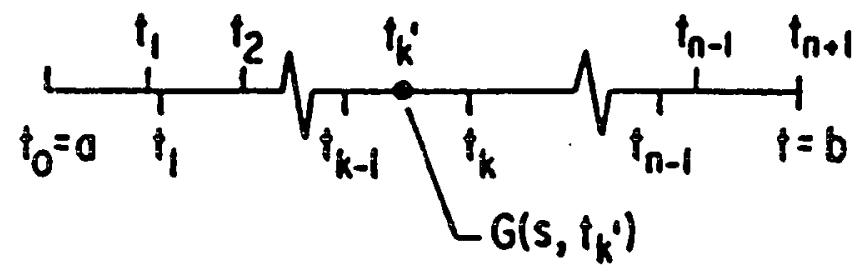

-Ito

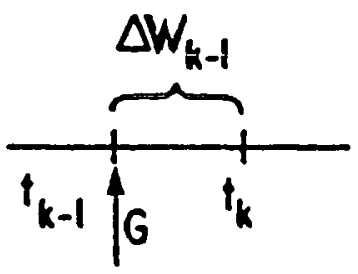

- Stratonovich

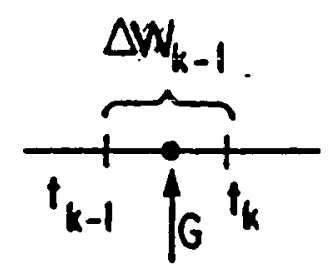

Figure 7. Distinction between the Ito and Stratonovich evaluations of the stochastic integral. 
implicit inclusion of the correlation characteristics between the integrand and the wiener increment (integrator).

The third integral in Eq. 4.2 is called the jump integral and may be defined in the Ito sense as a summation or counting integral (Snyder, 1975). If $N(t)$ incident points with marks $u_{j}$ have occurred on $\left[t_{0}, t\right)$ at instants $\tau_{j}$, the integral is given by the evaluation in Eq. 4.10 so that the integral actually represents a step function continuous everywhere except at points $\tau_{j}$.

Most of the concepts that will subsequently be utilized in this thesis have now been introduced. Other required ideas will be defined as they are needed in the subsequent derivations.

NUMERICAL METHODS FOR STOCHASTIC DIFFERENTIAL EQUATIONS

Analytical or closed-form solutions of the general SDE (Eq. 4.1 or 4.2) are not possible to obtain even if the jump term (involving the compound Poisson process) is absent. Numerical methods are, therefore, almost invariably sought to study SDE's. Even in this case, the available numerical approximations are only for the so-called Ito equations.

Performing a numerical approximation to an Ito integral involves two considerations. Firstly, it must be noted from property (iii) of the Wiener process that $E\left\{[d W(t)]^{2}\right\}=0(d t)$, so that $d W(t)=0(d t \cdot 5)$. Therefore, an approximation to $O\left(\Delta t^{n}\right)$ requires retaining terms to $O\left(d w^{2 n}\right)$. Secondiy, the approximation must preserve the nonanticipating property of the coefficients $a(X)$ and $b(X)$ in Eq. 4.2. For Ito equations, in which the jump term of $\mathrm{Eq} .4 .2$ is absent, consistent 
finite-difference approximations have been derived by Rao et al (1974) and Mil'shtein (1974). Other references pertaining to the subject of numerical solutions to Ito SDE's include Wright (1974), Durbin (1983) and Unny (1984). However, there does not appear to have been any study involving the numerical approximation of the general SDE which has the jump term included. Consequently, for the purpose of studying the sample paths of the SDE's derived in this thesis, a different approach, very approximate in nature, is adopted.

The numerical approximations here involve the use of an approximating sum to a stochastic integral as outlined in the preceding section (i.e., using an equation of the form given by Eq. 4.12). For instance, for an integral of the form:

$$
y_{t}=\int_{t_{0}}^{t} f(\tau) d W(\tau)
$$

in which $f(\tau)$ is a non-anticipating functional independent of the Wiener increment $d w(\tau)$, the interyal $\left[t_{0}, t\right]$ is divided into $k$ very small sub-intervals and $Y_{t}$ is approximated by the sum:

$$
Y_{k}=\sum_{i=1}^{k} f\left(\tau_{i}\right) \Delta W_{i}, \quad i=1,2, \ldots, k
$$

where $\tau_{j}=t_{0}+i h, h=\left(t-t_{0}\right) / k$ and $\Delta W_{j}=W\left(\tau_{j+1}\right)-W\left(\tau_{j}\right)$. $\Delta W_{i}$ is, of course, the Wiener increment, which can therefore be simulated by a normal random variable of mean zero and variance $B \Delta \tau i$, $\Delta \tau_{j}=\tau_{i+1}-\tau_{j} . \quad \beta^{2}$ is the variance parameter of the wiener process. In light of one of the considerations cited above, Eq. 4.19 
represents only an approximation to $0\left(\Delta \tau^{0.5}\right)$. The approximation is probably sufficient to reveal the characteristic behavior of the sample paths representing the solutions of the SDE's to be considered in this thesis.

The jump term is already given as a summation in Eq. 4.10. For sample paths involving this term, two other simulations to generate exponentially distributed interarrival times of the Poisson process and the values of the marks distributed according to $\operatorname{CDF} F U(u)$ would be required in addition to the normally distributed Wiener increments. 
CHAPTER V

\section{A STOCHASTIC DESCRIPTION OF SOIL MOISTURE}

A SIMPLE DYNAMIC SOIL MOISTURE MODEL

The derivation of a stochastic model for soil moisture follows directly from a simple, physically based dynamic soil moisture model presented by Koch et al (1986). The latter model describes the processes of infiltration, redistribution of soil moisture following a rainfall event, drainage of water out of the root zone and evapotranspiration (ET) of available water from the root zone both during and after the drainage process.

The dynamic soil moisture model described by Koch et al (1986) was developed on the basis of the sharp-wetting-front model of Green and Ampt (1911). The sharp-wetting-front model does, in fact, represent a useful practical simplification of the more rigorous hydrodynamic soil moisture model given by the Richards' equation as has been demonstrated by Dagan and Bresler (1983).

If one considers the soil moisture dymamics following a rainfall event, the initial situation is as depicted in Fig. 6(b) [Chapter III]. The distribution of soil water content is assumed to be a constant value, $\theta_{0}$, above the wetting front which is located at depth $Z_{0}$ below the ground surface, and a uniform initial value, $\theta_{i}$, below the front. Considering this initial "block" of soil moisture as a control 
volume, the following continuity equation, already given by Eq. 3.22 and repeated here for convenience, can be derived:

$$
Z_{0} \frac{d \theta}{d t}=-q(\theta)-E T(\theta)+f(t)
$$

where $f(t)$ is the rate of infiltration into the soil;

$\theta$ is the volumetric water content (moisture) per unit volume above the wetting front;

$q(\theta)$ is the rate of drainage across the control surface;

ET $(\theta)$ is the ET rate out of the control volume; and

$Z_{0}$ is the extent of the control volume below the surface at time $t=0$.

Eq. 5.1 describes the dynamics of soil moisture both during the time that there is water flow at the surface (i.e., during the rainfall event) and after it has ceased. In other words, $f(t)$ represents the flux term in the equation and leads to a complex soil moisture repletion process (or "wetting") at the surface.

The depth $z_{0}$ can be written in terms of the cumulative infiltration, $U$, resulting from the rainfall event as:

$$
Z_{0}=U /\left(\theta_{0}-\theta_{j}\right)
$$

where $\theta_{0}$ is the constant limiting value of moisture that obtains at the end of the rainfall event.

Following the rainfall event, the soil moisture "block" drains downward, due to capillary and gravitational forces, in a redistribution 
process that takes the form of a "stretching" of the initial profile, as illustrated in Fig. 6(b) [Chapter III]. Assuming, however, that gravity is the only significant force and that drainage can only occur when the soil water content is in excess of the residual value, $\theta_{r}$, the drainage rate can be expressed as:

$$
q(\theta)=K(\theta)
$$

in which $K(\theta)$ is the unsaturated hydraulic conductivity of the soil. Following experimental observations, Corey (1977) found that $K(\theta)$ could be approximated as:

$$
k(\theta)=\left\{\begin{array}{cl}
0, \quad \theta<\theta_{r} \\
k_{s}\left(\frac{\theta-\theta_{r}}{\theta_{s}-\theta_{r}}\right)^{n}, & \theta_{r} \leq \theta \leq \theta_{s}
\end{array}\right.
$$

where

$K_{S}$ is the hydraulic conductivity of the soil at natural saturation;

$\theta_{S}$ is the moisture at natural saturation; and

$n$ is an experimentally derived exponent.

The evapotranspiration (ET) process, which occurs simultaneously with the drainage process for $\theta \geqslant \theta_{r}$, may be represented by a simple expression proposed by Yaron et al (1973) and written as: 
$E T(\theta)= \begin{cases}K_{c}{ }_{c T}\left(\frac{\theta-\theta_{w p}}{\theta_{r}-\theta_{w p}}\right), & \theta_{w p} \leq \theta \leq \theta_{r} \\ K_{c} E T_{p}, & \theta_{r} \leq \theta \leq \theta_{s}\end{cases}$

in which $K_{c}$ is the crop consumptive use coefficient, $E T_{p}$ is the potential ET rate and $\theta_{w p}$ is the soil water content at the permanent wilting point, which is defined as the moisture below which plants can no longer extract water from the soil. The $\operatorname{ET}(\theta)$ function is depicted in Fig. 8. It must be noted here that ETp is an environmentally controlled random process.

Finally, the infiltration volume, $U$, expressed as a depth, represents the input to the model and is a function of both soil and climatic (in particular, rainfall) characteristics. The cumulative infiltration depends on the intensity and duration of the storm and may be obtained by integrating Eq. 3.20 over the storm duration giving:

$U=\left\{\begin{array}{l}r t_{d}, \quad t_{d}<t_{p} \\ r t_{p}+k_{s}\left(t_{d}-t_{p}\right)+S\left[\left(t_{d}-t_{p}+t_{c}\right)^{0.5}-\left(t_{c}\right)^{0.5}\right], t_{d} \geq t_{p}\end{array}\right.$

where $\beta$ has been taken as a factor equal to unity. Introducing the expressions for $q(\theta)$ from Eqs. 5.3 and 5.4, into Eq. 5.1, one obtains the following non-linear ordinary differential equation (ODE): 


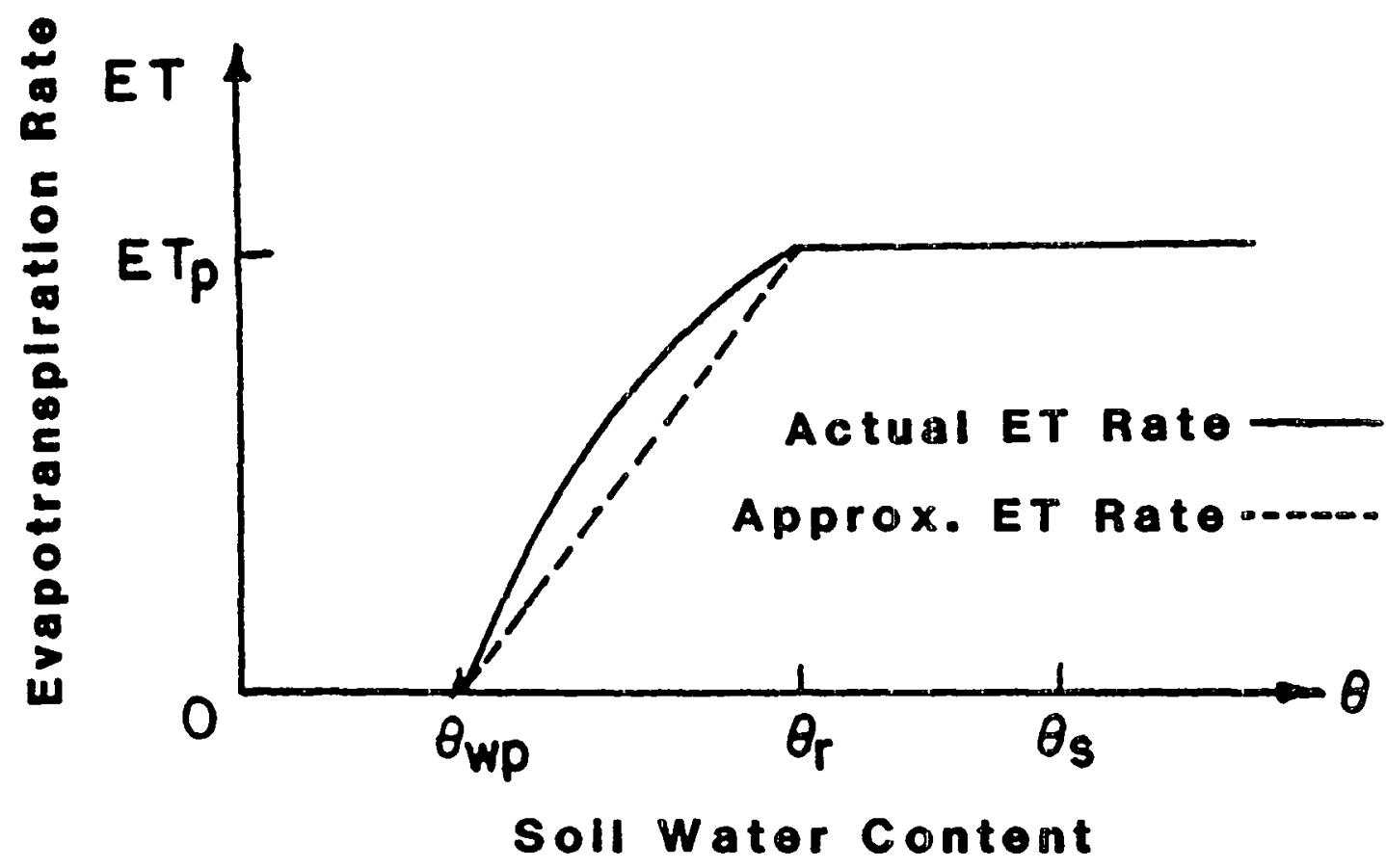

Figure 8. A simple evapotranspiration model. 


$$
Z_{0} \frac{d \theta}{d \dot{r}}=-K_{S}\left(\theta^{*}\right)^{n}-E T(\theta)+f(t)
$$

As observed in Koch et al (1986), this ODE does not have a closed form solution for $\theta(t)$ beyond the root zone depth $D$ for arbitrary values of the exponent $n$ in the drainage term. An analytical solution may, however, be easily obtained by linearizing this term. The linearization is accomplished by selecting the linear function in such a way that the areas under the actual and approximate functions are equal, thus preserving the drainage volume. This procedure is illustrated in Fig. 9 from which the "effective" value of the saturated hydraulic conductivity is given by:

$$
K_{e}=\frac{2 K_{s}}{n+1}
$$

The suitability of this approximation is demonstrated in Koch et al (1986). The linearized ODE, therefore, becomes:

$$
Z_{0} \frac{d \theta}{d t}=-K_{e}\left(\theta^{*}\right)-E T(\theta)+f(t)
$$

One additional important property to consider is the seasonal nature of the processes resulting in the depletion of soil moisture. Based on computer simulations, Koch and Mtundu (1986b) observed that, under a broad range of conditions, soil moisture tends to persist in either a "surplus" state such that there is drainage from the root zone, or in a "deficit" state where there is not sufficient soil moisture for drainage and thus ET is the only process depleting soil moisture. After Eagleson $(1978 a, b, c, d, e, f, g)$, these states might be classified as wet 


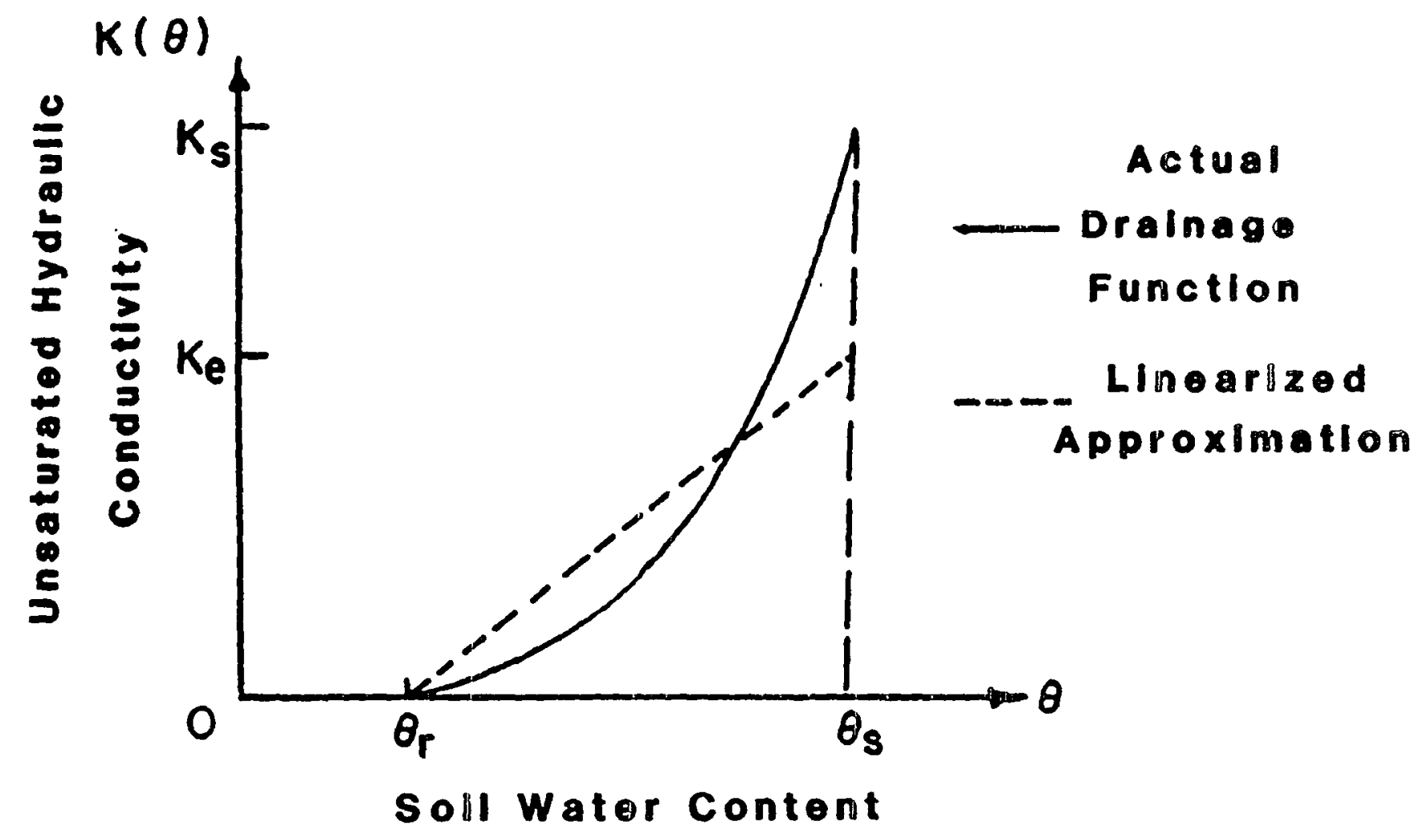

Figure 9. The drainage function and its linearized approximation. 
and dry seasons, respectively. Wet seasons tend to be characterized by frequent rainfall events and low ET rates, while the dry season exhibits relatively infrequent storms and high ET rates. There are, of course, transitional periods and not all hydrologic environments experience such well defined cases. However, this observation can be used to define limiting cases of soil moisture behavior in different environments.

Thus, in solving the linearized ODE, one needs to consider two cases. In the first case, one assumes that there is sufficient infiltration volume to produce drainage in the root zone; mathematically, $U>D\left(\theta_{r}-\theta_{j}\right)$ where $D$ is the total depth of the root zone. In the alternative case, the assumption is that $U<D\left(\theta_{r}\right.$ - $\left.\theta_{j}\right)$. The resulting ODE's are examined in the following section in which the stochastic formulation of the soil moisture dynamics problem is considered. However, before proceeding with the stochastic formulation, a summary of the deterministic model presented by Koch et at (1986) needs to be outlined since results of the simulations carried out using this model form the basis for comparison with the corresponding results obtained from the stochastic model.

The "Complete" Soil Moisture Simulation Model

The "complete" soil moisture dynamics model consists in accounting for all the processes occurring over finite periods of time in the root zone. The processes are "wetting" of the soil surface during a rainfall event, redistribution of the soil moisture "block" that is created during the "wetting" phase, and, finally, drainage and evapotranspiration (ET) that immediately follow the redistribution phase. 
The "Wetting" Phase. The "wetting" phase is responsible for initially raising the moisture level at the ground surface to the constant limiting value, $\theta_{0}$, which depends on the initial soil water content and the amount of infiltration that has occurred during a rainstorm.

The limiting value of $\theta_{0}$ is derived from a slightly modified Green-Ampt equation (Skaggs, 1982) written as:

$$
f=K\left(\theta_{0}\right)+\frac{K\left(\theta_{0}\right)\left[\theta_{0}-\theta_{i}\right] H_{c}}{U}
$$

where $K\left(\theta_{0}\right)$ is the hydraulic conductivity of soil at the limiting value, $\theta_{0}$, and $H_{C}$ is the capillary drive already defined in Chapter III (see Eq. 3.19). The rate of infiltration, $f=d U / d t$, before ponding time can be equated to the rainfall intensity, $r$, which is assumed to be constant. Upon applying the relation in Eq. 5.4 and re-arranging, Eq. 5.10 becomes:

$$
\theta_{0}=\theta_{r}+\left(\theta_{s}-\theta_{r}\right)\left\{\frac{r}{K_{s}\left[1+\left(\theta_{0}-\theta_{j}\right) H_{c} / U\right.}\right\}^{1 / n}
$$

Inasmuch as the second term on the right hand side of Eq. 5.11 contains a term with $\theta_{0}$, numerical methods must be sought to obtain the limiting value, $\theta_{0}$.

Redistribution, Drainage and Evapotranspiration Phases. The occurrence of the three phases, namely redistribution, drainage and ET, 
depends on whether or not there is sufficient infiltration volume to produce drainage out of the root zone. Assuming that there is, that is, $U>D\left(\theta_{r}-\theta_{j}\right)$, Eq. 5.7 is solved piecewise for $\theta$ in the redistribution, drainage and ET phases. At this point in time, the "wetting" phase is assumed to be over and that $f=0$. On further assuming that ET is negligible during the redistribution phase, the equation describing the moisture evolution for a wetting front at depth $Z<D$ obtained on the basis of Eq. 5.7 is:

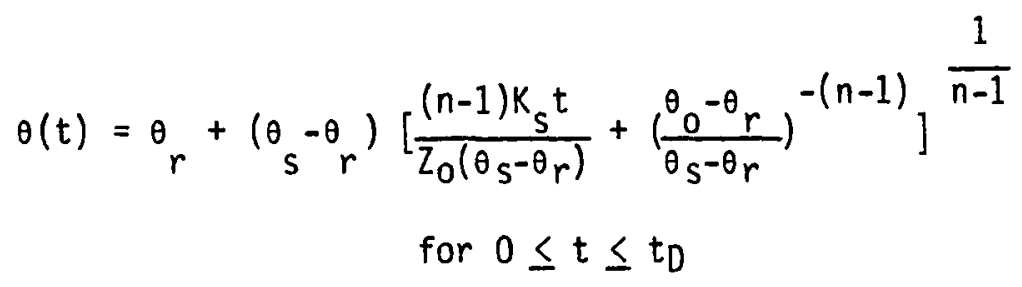

where $t_{D}$ is the time, measured from the beginning of the redistribution phase, required for the wetting front to reach the base of the root zone at depth $Z=D$. The time, $t_{D}$, is obtained by letting $\theta(t)=U / D+\theta_{j}$ in Eq. 5.12 and solving for $t=t_{D}$.

Once the wetting front has reached the depth $Z=0$, the soil moisture is uniform within the root zone and drainage occurs until $\theta=$ $\theta_{r}$. As indicated earlier, an analytical solution of Eq. 5.7 beyond the root zone depth is obtained by linearizing the drainage term, resulting in Eq. 5.9. The solution of Eq. 5.9 (with $f=0$ ) is:

$$
\begin{gathered}
\theta(t)=\theta_{r}+\left[\left(\theta_{D}-\theta_{r}\right)+\left(\theta_{S}-\theta_{r}\right) \frac{K_{C} E T_{p}}{K_{e}}\right] \exp \left[\frac{-K_{e}\left(t-t_{D}\right)}{D\left(\theta_{S}-\theta_{r}\right)}\right] \\
-\left(\theta_{S}-\theta_{r}\right) \frac{K_{C} E T_{p}}{K_{e}}, \quad t_{D} \leq t \leq t_{r}
\end{gathered}
$$


where $t_{r}$ is the time, measured from the start of the redistribution phase, when the moisture reaches the residual value, $\theta_{r}$ and $\theta_{D}$ is the moisture at time $t=t_{D}$.

Once the moisture is reduced to its residual value, drainage effectively stops but the ET process continues albeit at a depressed rate. The solution of Eq. 5.7 for this phase is:

$\theta(t)=\theta_{w p}+\left(\theta_{r}-\theta_{w p}\right) \exp \left[\frac{-K_{c} E T_{p}\left(t-t_{r}\right)}{D\left(\theta_{r}-\theta_{w p}\right)}\right], \quad t_{r} \leq t \leq t_{b}$

where $t_{b}$ is the arrival time of the following rainfall event (the time between storms).

For the other case in which $U<D\left(\theta_{r}-\theta_{j}\right)$, the redistribution phase is immediately followed by the ET phase. If one assumes that the time required for redistribution is negligible, inasmuch as this process occurs almost instantaneously, then the soil water content attains the value of $\theta_{0}=U / D+\theta_{i}$ immediately following the rainfall event. In this case, the evolution of water content occurs on the basis of the following solution of Eq. 5.7:

$\theta(t)=\theta_{w p}+\left(\theta_{0}-\theta_{w p}\right) \exp \left(\frac{-E T p t}{D\left(\theta_{r}-\theta_{w p}\right.}\right)$

The application of Eqs. 5.12 to 5.15 allows the tracking of soil moisture between rainfall events. 
Evaluation of the Simulation Model

Before using the simplified dynamic physical model of soil moisture as a basis for comparison with the stochastic model, one must first establish that the former behaves properly and produces results that closely approximate the observed behavior. A detailed evaluation of the simplified model has been presented by Koch et al (1986) where the performance was compared to laboratory drainage experiments and field moisture data for the ET-only phase collected in conjuction with an irrigation scheduling project. Also as part of the study, estimation of parameters was evaluated by comparing optimized parameter values with a priori estimates based on data obtained from routine soil surveys. A sample of the results using a priori parameter estimates is shown in Figure 10, which compares laboratory drainage rates with those computed using the simplified model. This figure illustrates that the approximate wetting, redistribution and drainage models represent the actual processes reasonably well. Further, Figure 11 presents time-variable soil moisture in a field along with predictions based on the simplified model in the deficit condition. Agreement here is also quite reasonable. Further comparisons are presented in Koch et al (1986). The results show that the simplified physical model provides a suitable tool for comparison with the stochastic model.

FORMULATION OF A STOCHASTIC DIFFERENTIAL EQUATION MODEL

Of important interest in this study are the stochastic properties of soil moisture. In order to investigate these properties, a slightly simplified form of the model described above is obtained as follows. 


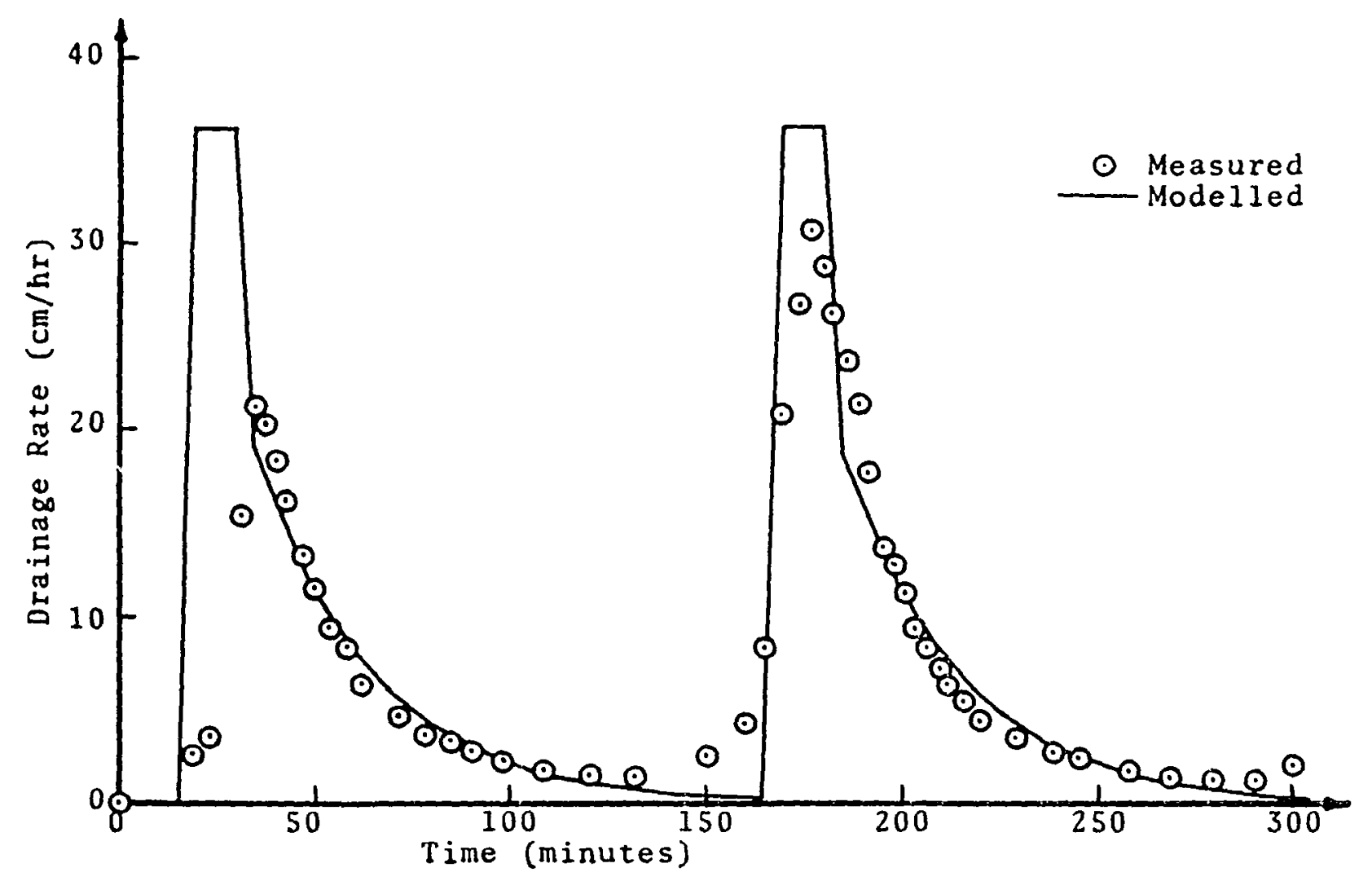

Figure 10. Comparison of simulated and observed sand flume drainage data 


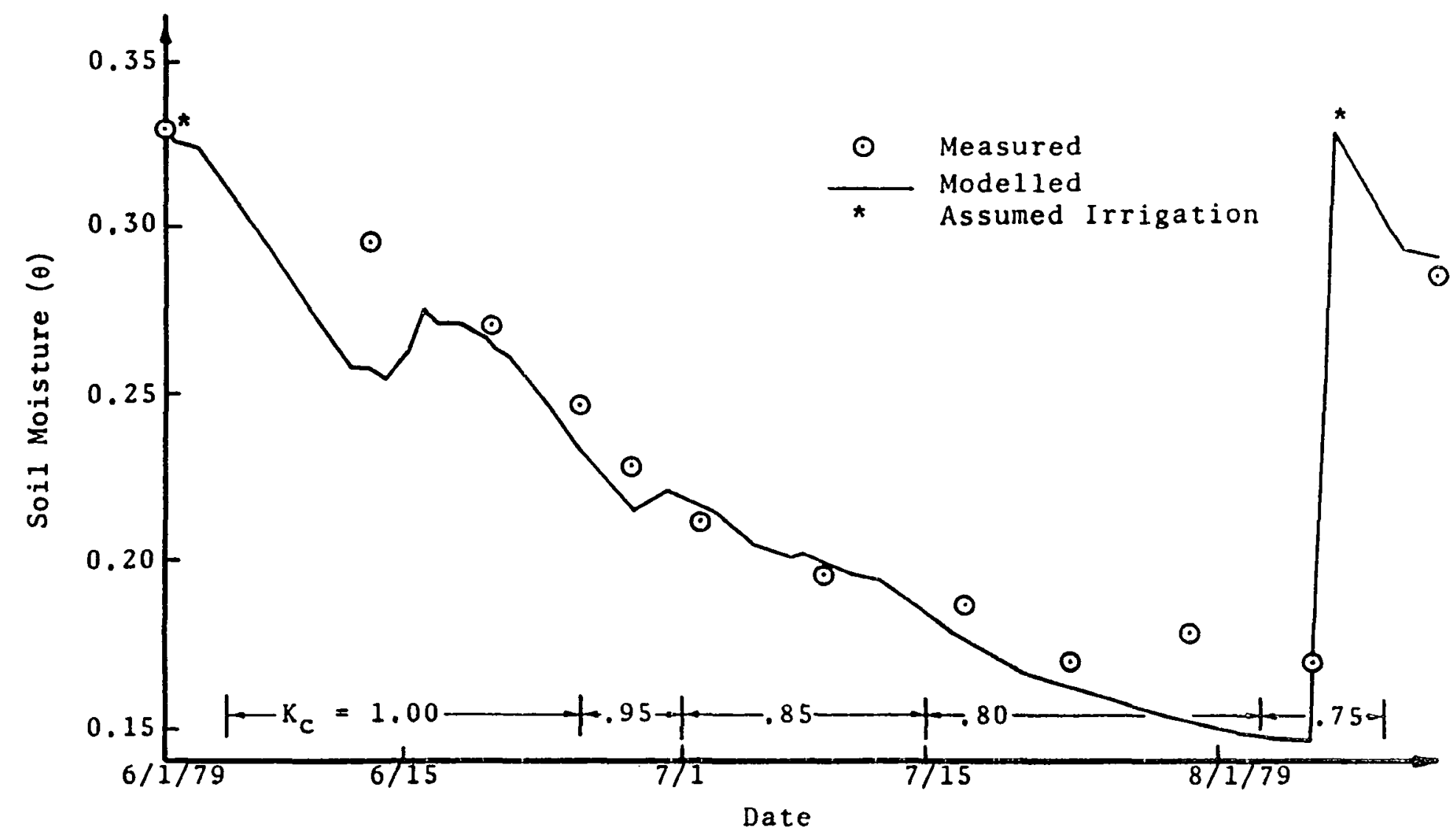

Figure 11. Simulation of soil moisture variation in a field of strawberries (Tualat in Valley Irrigation District, Oregon). crop coefficient varied during season as indicated. 
In the first $\mathrm{place}$, the constant-intensity rainfall events assumed to constitute the input to the model are random in their occurrence. Therefore, the amount of water infiltrated from these storm events constitutes a stochastic input into the soil water system. As such, the depth of infiltration in any given time interval $[0, t)$ will be dictated by the timing of the storm arrivals, the storm characteristics and the dynamics of the soil moisture depletion process. The occurrence of infiltration events will, therefore, be dependent on the same random process that dictates the occurrence characteristics of the storm events as long as the duration of the soil moisture repletion process is relatively short combared to the average time between the rainfall events.

A reasonable simplifying assumption with regard to the foregoing observation is that the wetting and redistribution processes in the soil dynamic model described above are considered to be instantaneous. As a first approximation, this is a fairly valid assumption as the redistribution process is generally a rapid one, taking on the order of a few hours compared to about a half-day or more for the other soil dynamic processes of drainage and evapotranspiration. The durations of these processes can also be compared to the interstorm periods which are, on average, much longer. The soil moisture is thus assumed to attain the instantaneous value of $\theta_{0}=\theta_{D}=\theta_{i}+U / D$ immediately following an infiltration event.

Considering the case in which any given infiltration event generates sufficient soil moisture to allow for drainage out of the root zone, the linearized ODE for the soil moisture evolution process takes 
the form:

$$
D \frac{d \theta}{d t}=-K_{e}\left(\frac{\theta-\theta_{r}}{\theta_{s}-\theta_{r}}\right)-K_{c} E T_{p}+f(t), \quad \theta_{r} \leq \theta \leq \theta_{s}
$$

in which all the variables and constants are as defined previously. This is an SDE with constant coefficients but random forcing due to a combination of ET and cumulative infiltration. Similarly, for the alternative case in which any given infiltration event does not generate sufficient volume to allow for drainage out of the root zone, the corresponding $O D E$ takes the form:

$$
D \frac{d \theta}{d t}=-K_{c} E T_{p}\left(\frac{\theta-\theta_{w p}}{\theta_{r}-\theta_{w p}}\right)+f(t), \quad \theta_{w p} \leq \theta \leq \theta_{r}
$$

where, again, the variables and coefficients are as defined previously. Here, we note that there is a random coefficient (ETp) and random forcing due to cummulative infiltration.

Eq. 5.16 will, henceforth, be used to represent the so-called "surplus" case by virtue of the fact that $U>D\left(\theta_{j}-\theta_{r}\right)$ for all infiltration events in a given time period $[0, t)$. In practice, this situation might be represented by a predominantly "wet" season which may, therefore, be loosely defined as one in which the storm characteristics are such that all infiltration events, on average, 
generate sufficient depth to allow for the drainage process to occur through the root zone and, therefore, also keep the moisture in the range $\left[\theta_{r}, \theta_{s}\right] . \quad E q .5 .17$, on the other hand, will be used to represent the "deficit" case which is likely to occur in a predominately "dry" season in which all the infiltration events, on average, generate insufficient depth to allow for the drainage process to occur through the root zone. That either the "surplus" or "deficit" scenario can occur has been corroborated (Koch and Mtundu, 1986a) by simulation runs on the soil moisture dynamic model presented in the last section.

The stochastic nature of the soil moisture dynamics problem arises from, among other things, the stochastic driving inputs to the dynamical systems as posed in Eqs. 5.16 and 5.17. As indicated earlier, the occurrence of the infiltration events corresponds to the random occurrence of rainfall events. Moreover, under the assumptions of independence and stationarity, the number of rainfall events in a given time interval $[0, t)$ may be modeled as a Poisson counting process (c.p.) $\{N(t), 0<t<\infty\}$ [see, for example, Eagleson 1978b]. In other words, the probability of having $n$ events in $[0, t)$ is given by:

$$
P[N(t)=n]=\frac{(\nu t)^{n} e^{-v t}}{n !}, n=1,2, \ldots
$$

where $v$ is the rate of the Poisson c.p.

The above assumption made of the rainfall process together with the assumption of "instantaneous" redistribution permits one to assert that the infiltration events will also follow a Poisson c.p. with the 
constant intensity of $\nu$. Inasmuch as each of the occurrence points of this c.p. carries the auxiliary random variable $U$ which represents the depth of infiltration generated by the rainfall-infiltration process, the obvious model for this input process is the compound Poisson process which can be formally represented by (see Snyder, 1975):

$$
f(t) d t=\int_{U} u M(d t, d u)
$$

where $M(d t, d u)$ is a compound Poisson measure of arrival rate $v$ and $u$ is the infiltration depth with distribution function $G_{y}(u)$ on space $U=[0, \infty)$ independent of the arrival process. A number of investigators have studied the nature of the distribution of $U$ (e.g. Cordova and Bras, 1981; Koch, 1982). For the purpose of this study, it is assumed that the infiltration depth can be reasonably represented by the distribution presented by Koch (1982). The distribution of infiltration depth, $U$, which is conditional on initial soil moisture, $\theta_{j}$, was formulated on the basis of a rainstorm of constant intensity, $r$, whose occurrence followed a Poisson process of rate $v$ and whose intensity and duration were both exponentially distributed with parameters $\beta$ and $\lambda$, respectively. The form of the distribution is:

$$
F_{U}(u)=1-\int_{0}^{r^{*}} \beta \exp \left(-\beta r-\frac{\lambda u}{r}\right) d r-\beta \exp \left(-\frac{\lambda s^{2}}{2 K_{s}^{2}}-\frac{\lambda u}{K_{s}}\right) \int_{r^{\star}}^{\infty} \exp [g(r, u)] d r
$$

where $g(r, u)=-B r-\frac{\lambda s^{2}}{2 r\left(r-k_{s}\right)}+\frac{\lambda S}{K_{s}}\left[\frac{s^{2}}{4 K_{s}^{2}}+\frac{s^{2}}{4\left(r-k_{s}\right)^{2}}+\frac{u}{K_{s}}\right]^{0.5}$

and $r^{*}=k_{s}+\frac{s^{2}}{2 u}$ 
All the other variables and coefficients are as defined previously.

The first and second moments of the distribution function (5.20) were determined, respectively, as:

$$
\begin{aligned}
E[U] & =\frac{1}{\lambda \beta}-\int_{K_{S}}^{\infty} B\left[\frac{r-K_{S}}{\lambda}-\frac{\pi}{\lambda} \frac{S}{2} \exp \left(A^{2}\right) \operatorname{erfc}(A)\right] \exp (-B r-B) d r \\
E\left[U^{2}\right] & =\frac{4}{(\lambda \beta)^{2}}-\beta \int_{K_{S}}^{\infty}\left[\frac{2\left(r^{2}-K_{S}^{2}\right)}{\lambda^{2}}-\frac{K_{S} S^{2}}{2 \lambda\left(r-K_{S}\right)}\right. \\
& \left.\left.-K_{S} \frac{\pi}{\lambda} \cdot \frac{\beta S}{2 \lambda}-\frac{s^{3}}{4\left(r-K_{S}\right)^{2}}\right) \operatorname{erfc}(A) \exp (\xi)\right] \exp (-\beta r-B) d r
\end{aligned}
$$

in which

$$
A=\frac{S \sqrt{ } \lambda}{2\left(r-K_{s}\right)}, \quad B=\frac{\lambda S^{2}}{2 r\left(r-K_{s}\right)}
$$

The other system driving process is evapotranspiration (ET). Unfortunately, the stochastic nature of ET is not adequately understood and, therefore, there are no universally acceptable stochastic models to describe its behavior. However, it has been suggested recently (Bodo and Unny, 1985) that ET could be modeled as a wide-band noise with stationary zero-mean white Gaussian fluctuations superposed on a constant mean rate, that is: 
$\varepsilon=\bar{\varepsilon}+\varepsilon^{\prime}$

where $\bar{\varepsilon}$ is the mean rate of ET and $\varepsilon^{\prime}$ is the fluctuating part of ET. Hence, the ET process may be presented by:

$$
\varepsilon d t=\bar{\varepsilon} d t+\beta d W_{t}
$$

where the Gaussian white noise $\varepsilon^{\prime}$ is perceived as the symbolic derivative of the normalized Wiener process $\left\{W_{t}, t>0\right\}$, that is:

$$
\epsilon^{\prime}=\frac{{ }^{B d W} t}{d t}
$$

which by formal manipulation yields:

$$
d W(t)=\left(\varepsilon^{\prime} / \beta\right) d t
$$

$\beta^{2}$ is the variance parameter of the white Gaussian noise. Upon substitution of the stochastic driving inputs, as given in Eqs. 5.19 and 5.24, into Eqs. 5.16 and 5.17, one obtains for the "surplus" case:

$$
d \theta=\left(\alpha \theta_{r}-\alpha \theta-\psi \bar{\varepsilon}\right) d t-\psi B d W_{t}+\int_{U} \frac{u}{D} M(d t, d u)
$$

in which $\alpha=\frac{K_{e}}{D\left(\theta_{S}-\theta_{r}\right)}$ and $\psi=\frac{K_{c}}{D}$ 
For the "deficit" case:

$$
\begin{array}{r}
d \theta=\left(\delta \theta_{w p}-\delta \theta\right) d t+\left(\gamma \beta \theta_{w p}-\gamma \beta \theta\right) d w_{t}+\int_{U} \frac{u}{D} M(d t, d u) \\
\text { in which } \delta=\frac{K_{c} \bar{\varepsilon}}{D\left(\theta_{r}-\theta_{w p}\right)} \text { and } \gamma=\frac{K_{C}}{D\left(\theta_{r}-\theta_{w p}\right)}
\end{array}
$$




\section{CHAPTER VI}

SAMPLE FUNCTIONS AND MOMENTS OF SOIL MOISTURE

$$
\text { "SURPLUS" CASE }
$$

For convenience, Eq. 5.27 is re-written as:

$$
d \theta_{t}=a_{0} d t+a_{1} \theta_{t} d t+b_{0} d w_{t}+\int_{U} c_{0} u M(d t, d u)
$$

where:

$$
\begin{array}{ll}
a_{0}=\alpha \theta_{r}-\psi \vec{\varepsilon}, & a_{1}=-\alpha, \\
b_{0}=-\psi \beta, & c_{0}=1 / 0
\end{array}
$$

Eq. 6.1 is a special case of a more general stochastic differential equation (4.1) which may be written as:

$$
d \theta_{t}=a(\theta, t) d_{t}+b(\theta, t) d W_{t}+\int_{U} c(\theta, t, u) M(d t, d u,)
$$

in which:

$$
a=a_{0}+a_{1} \theta, b=b_{0}+b_{1} \theta \text { and } c=\left(c_{0}+c_{1} \theta\right) u \text {. }
$$

For Eq. 6.2, Gihman and Skorohod (1972) derived a sample function representation which is given by: 


$$
\begin{gathered}
\theta_{t}=\theta_{0} \xi\left(t, t_{0}\right)+\int_{t_{0}}^{t} \xi(t, \tau)\left(a_{0}-b_{0} b_{1}\right) d \tau+\int_{t_{0}}^{t} \xi(t, \tau) b_{0} d w \\
+\int_{t_{0}}^{t} \int_{\tau} \xi(t, \tau) \frac{c_{0} u}{1+c_{1} u} M(d \tau, d u)
\end{gathered}
$$

This solution process is obtained on the assumption that $c_{1} u>-1$ and by defining the kernel function as:

$$
\xi(t, \tau)=\exp \left\{\int_{\tau}^{t}\left(a_{1}-0.5 b_{1}^{2}\right) d \sigma+\int_{\tau}^{t} b_{1} d w_{\sigma}+\int_{\tau}^{t} \int_{U} \ln \left(1+c_{1} u\right) M(d \sigma, d u)\right\}
$$

Noting that $b_{1}=c_{1}=0$ in the special case considered in Eq. 6.1, one obtains the following solution process for the "surplus" situation:

$$
\begin{aligned}
\theta(t)= & \theta_{0} \xi\left(t, t_{0}\right)+\int_{t_{0}}^{t} \xi(t, \tau) a_{0} d \tau+\int_{t_{0}}^{t} \xi(t, \tau) b_{0} d W \\
& +\int_{t_{0}}^{\tau} \int_{u} \xi(t, \tau) c_{0} u M(d \tau, d u)
\end{aligned}
$$

where the kernel function is simply given by:

$$
\xi(t, \tau)=\exp \left\{\int_{\tau}^{t} a_{1} d \sigma\right\}=e^{a_{1}(t-\tau)}=e^{-\alpha(t-\tau)}
$$

With $t_{0}=0$, one obtains: 


$$
\begin{aligned}
\theta(t)= & \theta_{0} e^{-\alpha t}+\frac{a_{0}}{\alpha}\left(1-e^{-\alpha t}\right)+b_{0} \int_{0}^{t} e^{-\alpha(t-\tau)} d W_{\tau} \\
& +\int_{0}^{t} \int_{U} c_{0} u e^{-\alpha(t-\tau)} M(d \tau, d u)
\end{aligned}
$$

The last term in Eq. 6.7 is zero for $N(t)=0$; otherwise it has the evaluation given by (e.g. Snyder, 1975):

$$
\int_{0}^{t} \int_{0} c_{0} u e^{-\alpha(t-\tau)} M(d \tau, d u)=\sum_{j=1}^{N(t)} c_{0} u_{j} e^{-\alpha\left(t-\tau_{j}\right)}, N(t) \geq 1
$$

where $N(t)$ is the number of Poissonian impulses occurring during $[0, t)$. On substituting for $a_{0}, b_{0}$ and $c_{0}$, one obtains, finaliy, the following sample function representation of the evolution process of soil moisture:

$$
\begin{aligned}
& \theta(t)=\theta_{r}+\left(\theta_{0}-\theta_{r}\right) e^{-\alpha t}-n\left(1-e^{-\alpha t}\right) \\
& -\frac{K_{c} B}{D} \int_{0}^{t} e^{-\alpha(t-\sigma)} d W_{\sigma}+\sum_{j=1}^{N(t)} \frac{u_{j}}{D} e^{-\alpha\left(t-\tau_{j}\right)}
\end{aligned}
$$

in which $\alpha=\frac{K_{e}}{D\left(\theta_{s}{ }^{\left.-\theta_{r}\right)}\right.}$ and $\eta=\frac{K_{c}}{K_{e}}\left(\theta_{s}-\theta_{r}\right) \bar{\varepsilon}$ 
Except for the integral involving the Wiener process, Eq. 6.9 is the same solution process obtained by Koch and Mtundu (1986a) using a different approach. This approach is briefly described later in Chapter VII.

For a complete description of the sample function, it is necessary that the Ito stochastic intergral in Eq. 6.9 be numerically evaluated.

\section{"DEFICIT" CASE}

In order to compare with the more general SDE given by Eq. 6.2, Eq. 5.28 is re-written in the form:

$$
d \theta_{t}=a_{0} d t+a_{1} \theta_{t} d t+b_{0} d W_{t}+b_{1} \theta_{t} d W_{t}+\int_{U} c_{0} u M(d t, d u)
$$

where:

$$
\begin{aligned}
& a_{0}=\delta \theta_{w p}, \quad b_{0}=\gamma \beta \theta_{w p}, \quad c_{0}=\frac{1}{D} \\
& a_{1}=-\delta, \quad b_{1}=-\gamma \beta
\end{aligned}
$$

In this case, only $c_{0}=0$ so that the kernel function $(6.4)$ becomes

$$
\xi(t, \tau)=\exp \left\{\int_{\tau}^{t}\left(a_{1}-0.5 b_{1}^{2}\right) d \sigma+\int_{\tau}^{t} b_{1} d W_{\sigma}\right\}
$$

Thus, according to Eq. 6.3, the solution process for the "deficit" situation is: 


$$
\begin{aligned}
\theta(t)=\theta_{0} \xi(t, \tau) & +\int_{0}^{t} \xi(t, \tau)\left(a_{0}+\dot{b}_{0} \dot{b}_{1}\right) d \tau+\int_{0}^{t} \xi(\dot{\tau}, \tau) \dot{b}_{0} d w_{\tau} \\
& +\int_{0}^{t} \int_{U} \xi(t, \tau) c_{0} u M(d \tau, d u)
\end{aligned}
$$

where $\xi(t, \tau)$ is given by Eq. 6.11. Substituting for $a_{0}, b_{0}, b_{1}, c_{0}$, one obtains:

$$
\begin{aligned}
& \theta(t)=\theta_{0} \exp \left[-\left(\delta+\frac{\gamma^{2} \beta^{2}}{2}\right) t-\gamma \beta \int_{0}^{t} d W W_{\sigma}\right\} \\
& +\int_{0}^{t} \theta_{W p}\left(\delta-\gamma^{2} \beta^{2}\right) \exp \left\{-\left(\delta+\frac{\gamma^{2} \beta^{2}}{2}\right)(t-\tau)-\gamma \beta \int_{\tau}^{t} d W\right\} d \tau \\
& +\int_{0}^{t} \gamma \beta \theta_{W p} \exp \left\{-\left(\delta+\frac{\gamma^{2} \beta^{2}}{2}\right)(t-\tau)-\gamma \beta \int_{\tau}^{t} d W W_{\sigma}\right\} d W \\
& +\int_{0}^{t} \int_{U} \frac{u}{D} \exp \left\{-\left(\delta+\frac{\gamma^{2} \beta^{2}}{2}\right)(t-\tau)-\gamma \beta \int_{\tau}^{t_{\tau}} d W_{\sigma}\right\} M(d \tau, d u)
\end{aligned}
$$

Again, numerical procedures must be sought in order to determine the sample function representing the soil moisture evolution process.

\section{ILLUSTRATION OF SOLUTION BEHAVIOR}

In order to evaluate the stochastic dynamic model of soil moisture derived in the preceding section, the solutions given in Eqs 6.9 and 6.13 were numerically integrated for a clay loam soil and two different climatic conditions. Blacksburg, Virginia, located in the humid, east central USA was selected to represent a climate in which the "surplus" case would prevail whereas Denver, Colorado, located in the 
semi-arid western USA, was used as an example to simulate the "deficit" case. The characteristics of these two climatic conditions are presented in Table I, whereas typical values representing subsurface zone characteristics of the selected soil are given in Table II.

As indicated earlier, the relationship between evapotranspiration and the Brownian motion model is not adequately understood to allow for a parametrization of the stochastic model selected herein to describe its behavior. Difficulty in parametrization arises in connection with a proper choice of the variance parameter, $\beta^{2}$, of the white Gaussian noise used to model the fluctuating component of ET (see Eq. 5.24). For the purposes of this illustration, two values of $\beta^{2}$, an order of magnitude apart, were selected for each of the "surplus" and "deficit" cases.

The procedure adopted for the numerical integrations carried out in this study is as outlined in Chapter IV.

"Surplus" Case

With a value of $0.1 \mathrm{~cm}^{2} /$ day selected for the variance parameter, $\beta^{2}$, a portion of the sample function of the moisture evolution process for the "surplus" case was obtained and plotted as shown in Fig. 12. This sample path may be recognized to be that of a filtered Poisson process. The Poissonian "jumps" are clearly evident from this figure, occurring at the times of the impulsive inputs of infiltration, as are some fluctuations in exponential decay of the process between the "jumps". Fig. 13 gives an amplified version of one section of the sample function to more closely illustrate random 
TABLE I

MAEN INTENSITY $(1 / \beta)$, MEAN DURATION $(1 / \lambda)$, MEAN INTER-STORM PERTODS $(1 / \nu)$ OF RAINFALL EVENTS AND MEAN POTENTIAL

EVAPOTRANSPIRATION RATES FOR

TWO SELECTED CLIMATES

\begin{tabular}{|c|c|c|c|c|}
\hline Location & $\begin{array}{c}1 / \beta \\
(\mathrm{cm} / \text { day })\end{array}$ & $\begin{array}{c}1 / \lambda \\
\text { (day) }\end{array}$ & $\begin{array}{l}1 / v \\
\text { (day) }\end{array}$ & $\begin{array}{c}\text { ETp } \\
(\mathrm{cm} / \text { day })\end{array}$ \\
\hline Bl acksburg, $\mathrm{Va}^{1}$ & 5.08 & 0.300 & 3.48 & 0.0624 \\
\hline $\begin{array}{l}\text { Denver, } \mathrm{Co}^{2} \\
\text { ("Wet" Season) }\end{array}$ & 3.75 & 0.240 & 3.34 & 0.310 \\
\hline $\begin{array}{l}\text { Denver, } \mathrm{Co}^{2} \\
\text { ("Dry" Season) }\end{array}$ & 3.75 & 0.100 & 3.83 & 0.630 \\
\hline
\end{tabular}


TABLE II

TYPICAL VALUES REPRESENTING SUBSUFACE ZONE CHARACTERISTICS

\begin{tabular}{lcccccc}
\hline Soil Texture & $\theta_{\text {wp }}$ & $\theta_{r}$ & ${ }^{\theta_{s}}$ & $\begin{array}{c}K_{s} \\
(\mathrm{~cm} / \text { day })\end{array}$ & $n$ & $\begin{array}{c}\mathrm{H}_{\mathrm{C}} \\
(\mathrm{cm})\end{array}$ \\
\hline Clay Loam & 0.21 & 0.37 & 0.49 & 2.45 & 10 & 19.0 \\
Loam & 0.15 & 0.31 & 0.45 & 7.92 & 8 & 21.8 \\
Sandy Loam & 0.05 & 0.21 & 0.40 & 26.2 & 6 & 64.0 \\
\hline
\end{tabular}




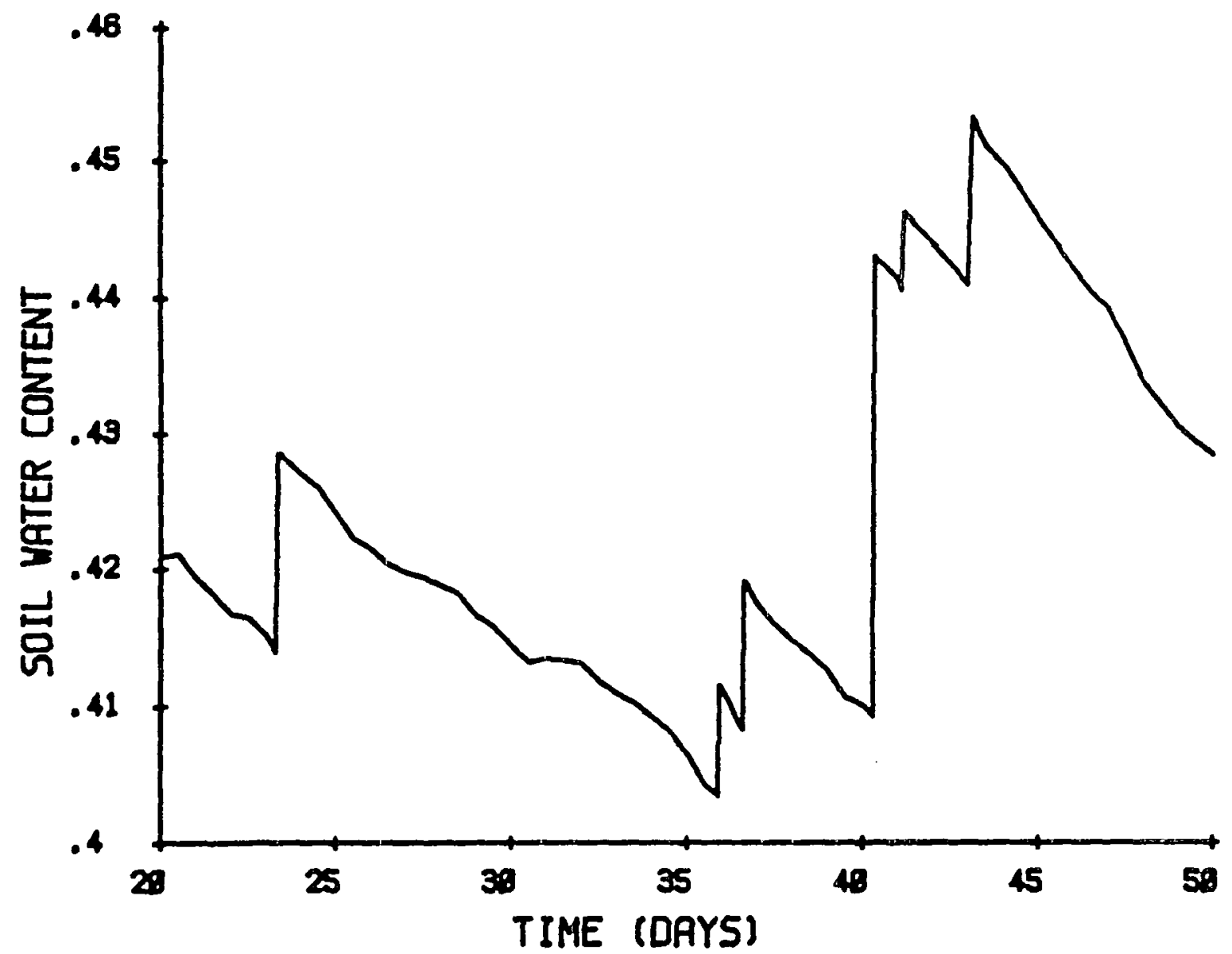

Figure 12. A section of the sample function of soil moisture evolution process as given by Eq. 6.9 with $B^{2}=0.1 \mathrm{~cm}^{2} /$ day. 


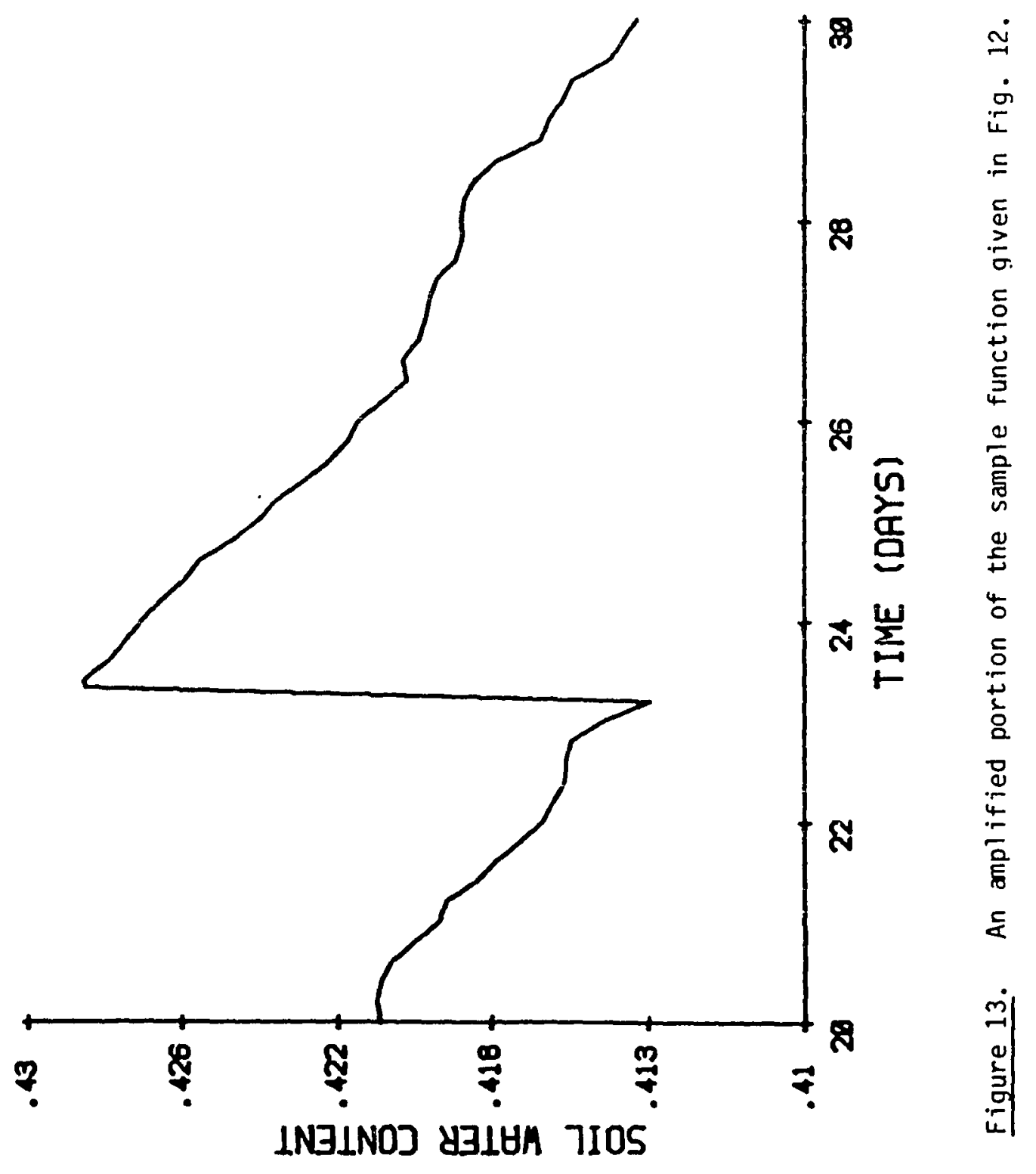


fluctuations in the "decay" periods which result from depletions of soil moisture due to the random ET process.

When a value of $B^{2}=1.0 \mathrm{~cm}^{2} /$ day was selected for the variance parameter, the sample function generated using Eq. 6.9 had the appearance given in Fig. 14. Clearly, the random fluctuations due to ET were amplified to the extent that they effectively masked the Poissonian "jumps"; therefore the resulting realization of the process is not physically realistic since there can be no increase in $\theta(t)$ due to $E T$. This observation points to the necessity of appropriately selecting a value of $\beta^{2}$ or, what is better, establishing a physically based method of determining its value.

"Deficit" Case

In this case, the value of parameter $\beta^{2}$ which resulted in a physically meaningful process was $1.0 \mathrm{~cm}^{2} /$ day. A portion of the resulting sample function (also in the form of a filtered Poisson process) obtained by integrating Eq. 6.13 using the appropriate parameter values is shown in Fig. 15. The amplified version is given in Fig. 16. Both figures clearly show where the Poissonian "jumps" occur as well as the ET-controlled random exponential decay periods between the "jumps". When the value of $\beta^{2}$ is an order of magnitude lower, the random fluctuations are no longer evident and the mean rate of ET controls depletion as shown in Fig. 17, which was obtained with $\beta^{2}=$ $0.1 \mathrm{~cm}^{2} /$ day. 


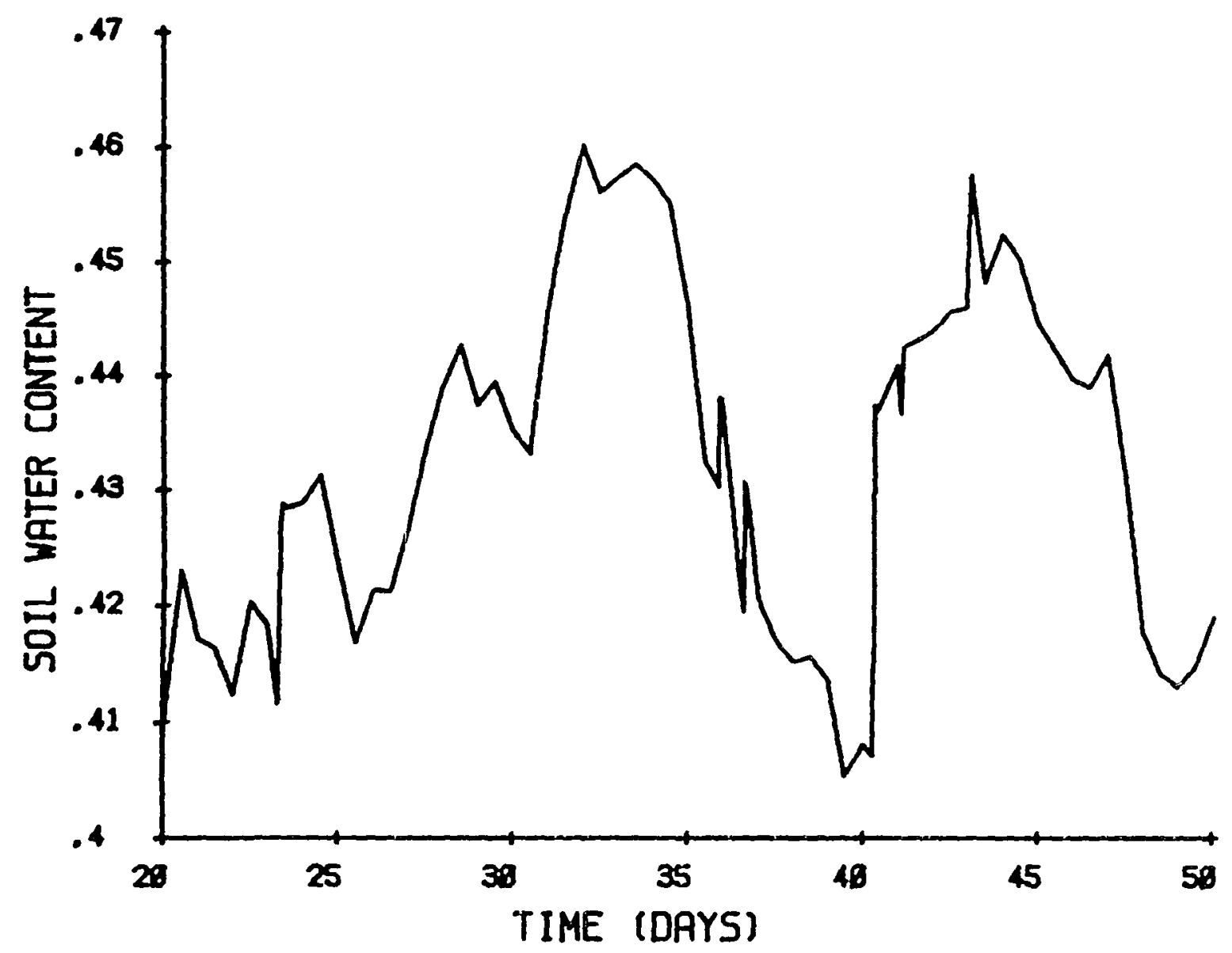

Figure 14. A section of the sample function of soil moisture evolution process as given by Eq. 6.9 with $\beta^{2}=1.0 \mathrm{~cm}^{2} /$ day. 


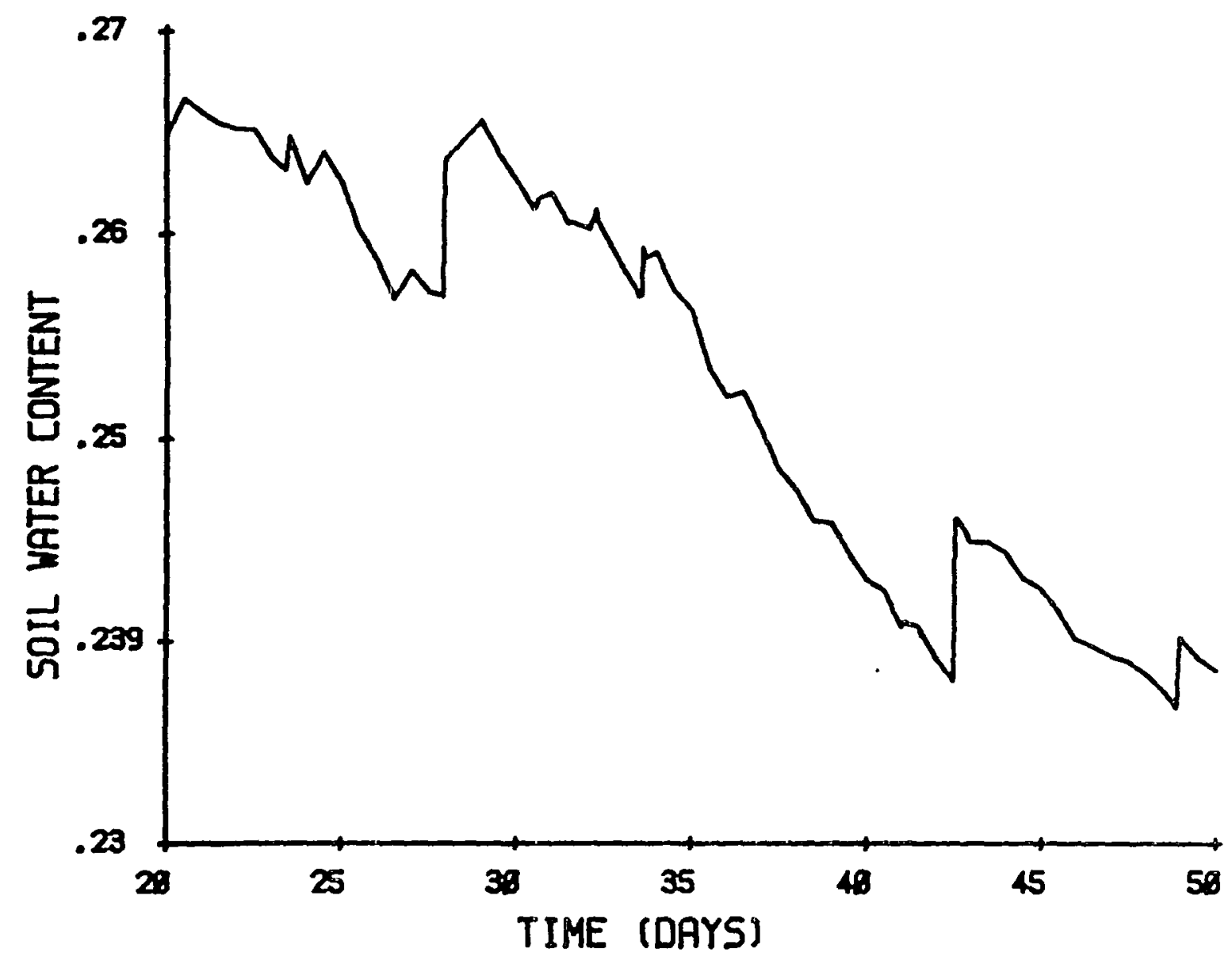

Figure 15. A section of the sample function of soil moisture evolution process as given by Eq. 6.13 with $\beta^{2}=1.0 \mathrm{~cm}^{2} /$ day. 


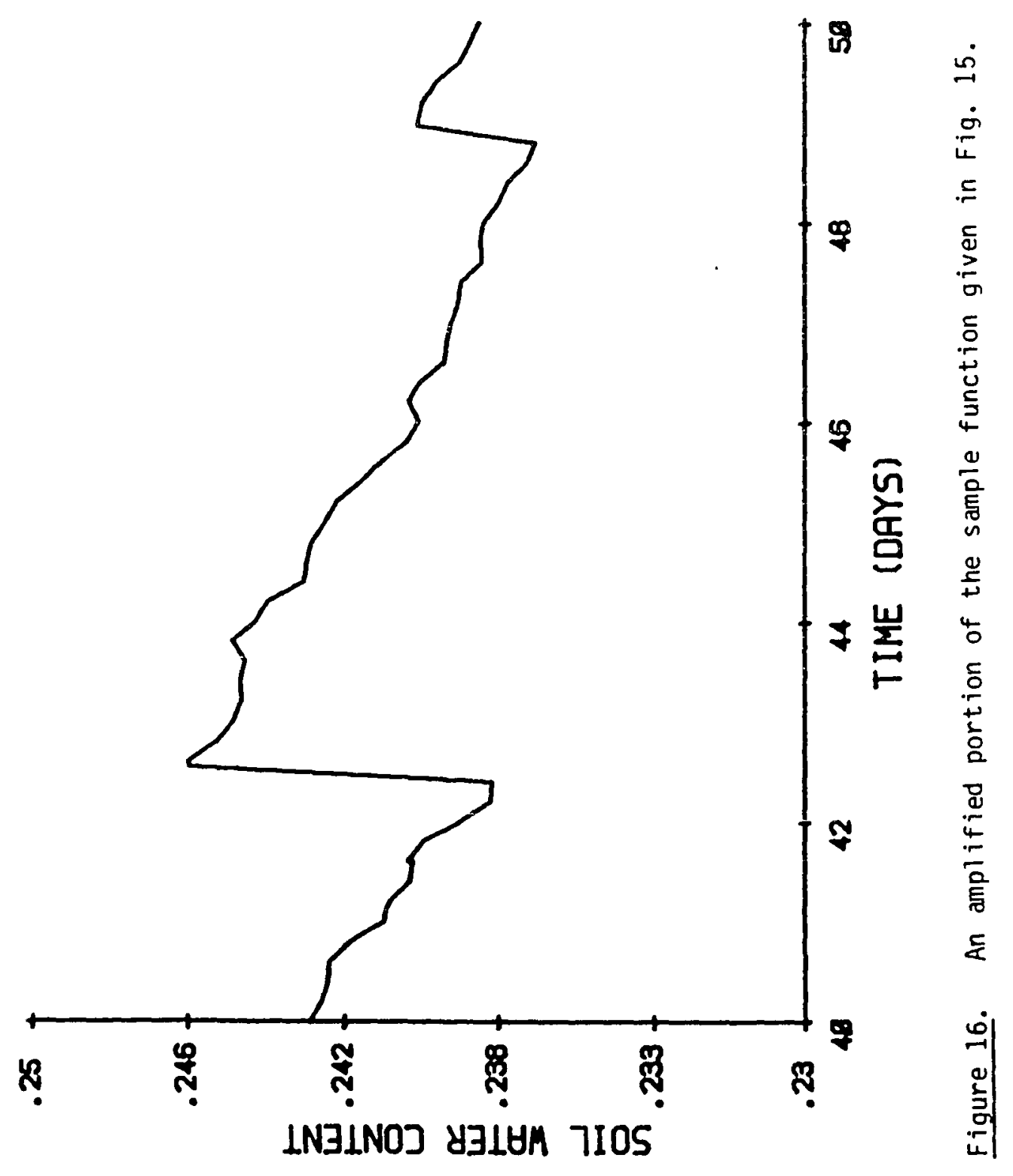




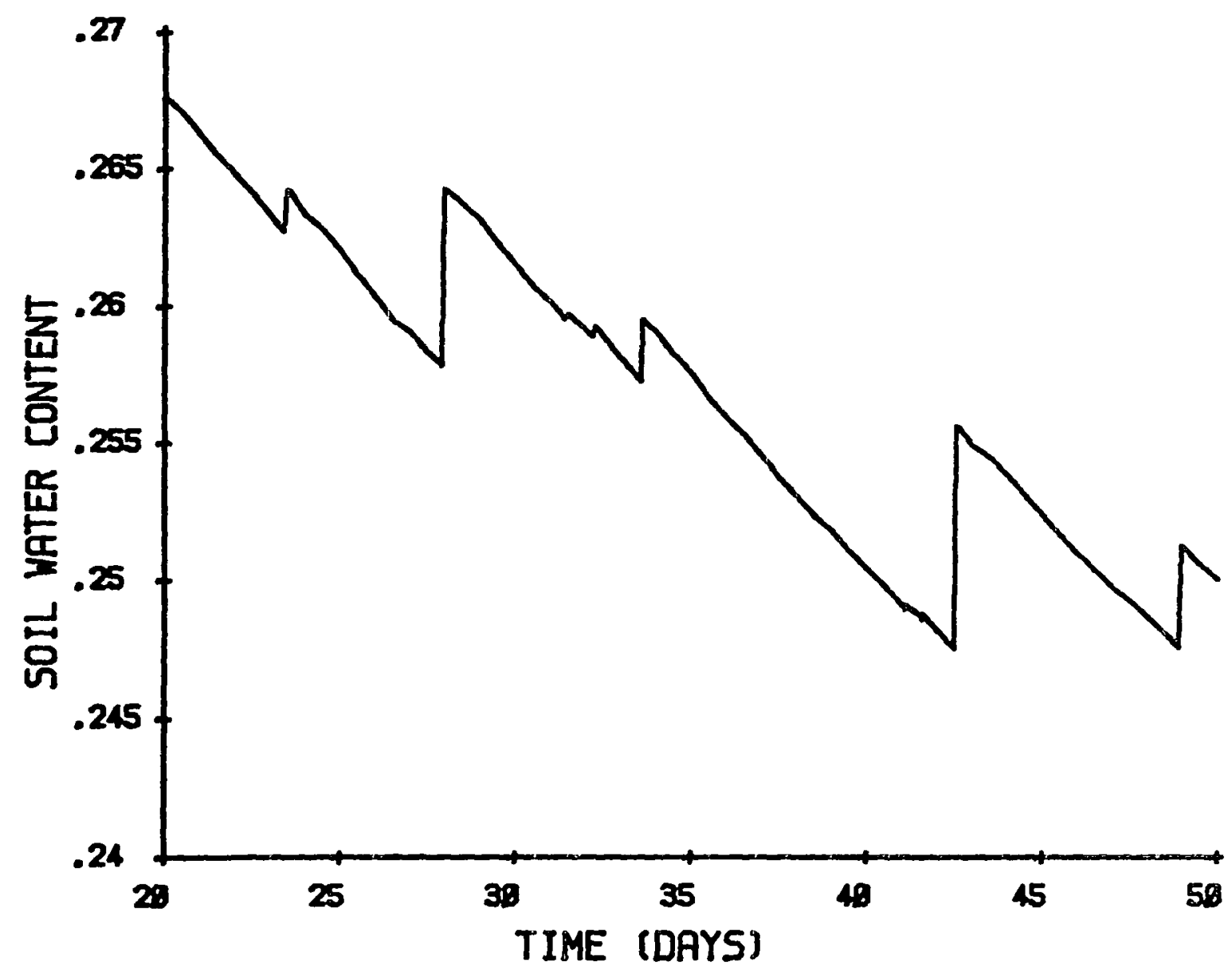

Figure 17. A section of the sample function of soll moisture evolution process as given by Eq. 6.13 with $\beta^{2}=0.1 \mathrm{~cm}^{2} /$ day. 


\section{MOMENT PROPERTIES}

In many cases, it may be sufficient to obtain the first few moments of the stochastic process representing the solutions of the SDE's. Here, expressions for the mean, variance and correlation structure of soil moisture in both the "surplus" and "deficit" cases are obtained. For the simplified models, analytical results relating the stochastic behavior of soil moisture to parameters describing the climatic and physical system are possible. From the moment equations, it is possible to identify the significance of the governing physical and climatic processes in the stochastic behavior of soil moisture.

"Surplus" Case

The first moment (or mean) of the solution process (6.9) is obtained as:

$$
\begin{aligned}
E[\theta(t)] & =E\left[\theta_{r}\right]+E\left[\left(\theta_{0}-\theta_{r}\right) e^{-\alpha t}\right]-E\left[n\left(1-e^{-\alpha t}\right)\right] \\
& \left.-E\left[\frac{K_{C} \beta}{D} \int_{0}^{t} e^{-\alpha(t-\sigma)_{d W_{0}}}\right]+E\left[\sum_{j=1}^{N(t)} \frac{U_{j}}{D} e^{-\alpha(t-\tau}\right)_{j}\right]
\end{aligned}
$$

The first three terms are determined in a straight forward manner. The fourth term is zero because of the independence of the integrand and integrator (the Wiener increment, $d W_{\sigma}$ ) when the stochastic integral is interpreted in the Ito sense. The last term can be evaluated by observing that the expectation operand is a filtered Poisson process for which the first moment equation is presented by Parzen (1962, Section 4.5). The resulting expression for the mean process is: 


$$
E[\theta(t)]=\theta_{r}+\left(E\left[\theta_{0}\right]-\theta_{r}\right) e^{-\alpha t}-n\left(1-e^{-c t}\right)+\frac{\nu E[U]}{\alpha D}\left(1-e^{-c t}\right)
$$

The mean for the stationary process, in which the effects of the initial conditions have effectively diminished, is obtained by letting $t$ tend to infinity. Upon substitution of $\alpha$ and $\eta$, the result is:

$$
E[\theta(t)]=\theta_{r}-\frac{K_{C}}{K_{e}}\left(\theta_{S}-\theta_{r}\right) \bar{\varepsilon}+\frac{\nu E[U]\left(\theta_{S}-\theta_{r}\right)}{K_{e}}
$$

With the distribution of $U$ selected earlier, the expectation $E[U]$ is as given by Eq. 5.21 .

The autocovariance of the solution process for two time instants $\tau$ apart can be derived directly from the definition:

$$
\Gamma(t, t+\tau)=\operatorname{Cov}[\theta(t), \theta(t+\tau)]
$$

A11 the terms in Eq. 6.9 are independent of one another; therefore, a11 pairwise covariances are zero as are those of the constants, leaving:

$$
\begin{aligned}
& \Gamma(t, t+\tau)=\operatorname{Cov}\left[\left(\theta_{0}-\theta_{r}\right) e^{-\alpha t},\left(\theta_{0}-\theta_{r}\right) e^{-\alpha(t+\tau)}\right] \\
& +\operatorname{Cov}\left[\frac{K_{C} \beta}{D} \int_{0}^{t} e^{-\alpha(t-\sigma)} d W, \frac{K_{C} \beta}{D} \int_{0}^{t+\tau} e^{-\alpha(t+\tau-\sigma)} d W\right] \\
& \left.+\operatorname{Cov}\left[\sum_{j=1}^{N(t)} \frac{U_{j}}{D} e^{-\alpha\left(t-\tau_{j}\right)}, \sum_{j=1}^{N(t+\tau)} \frac{U_{j}}{D} e^{-\alpha(t+\tau-\tau}\right)_{j}\right]
\end{aligned}
$$


The first term reduces to:

$$
\operatorname{Cov}\left[\left(\theta_{0}-\theta_{r}\right) e^{-\alpha t},\left(\theta_{0}-\theta_{r}\right) e^{-\alpha(t+\tau)}\right]=e^{-\alpha(2 t+\tau)} \operatorname{Var}\left(\theta_{0}-\theta_{r}\right)
$$

The second term may be written :

$$
\begin{aligned}
\operatorname{Cov}\left[\frac{K_{C} \beta}{D} I(t), \frac{K_{C} \beta}{D} I(t, t+\tau)\right] & =E\left[\frac{K_{C}^{2} \beta^{2}}{D^{2}} I(t) I(t+\tau)\right] \\
& -E\left[\frac{K_{C} \beta}{D} I(t)\right] E\left[\frac{K_{C} \beta}{D} I(t+\tau)\right]
\end{aligned}
$$

where $I(t)=\int_{0}^{t} e^{-\alpha(t-\sigma)} d W_{\sigma}$

The expectation of each of the terms in the product on the right-hand side of the equation is zero because of the independence of the integrand and integrator when the stochastic integrals are interpreted in the Ito sense. Using Eq. 4.17 and noting that $W_{t}$ has been taken to be the normalized Wiener process (with unit variance), this second term thus reduces to:

$$
\begin{aligned}
\operatorname{Cov}\left[\frac{K_{c} \beta}{D} I(t), \frac{K_{c} \beta}{D} I(t+\tau)\right] & =\frac{K_{C}^{2} \beta^{2}}{D^{2}} \int_{0}^{t} e^{-\alpha(t-\sigma)} e^{-\alpha(t+\tau-\sigma)} d \sigma \\
& =\frac{K_{c}^{2} \beta^{2}}{D^{2}}\left(1-e^{-2 \alpha t}\right) \frac{e^{-\alpha \tau}}{2 \alpha}
\end{aligned}
$$

The third term in Eq. 6.18 is evaluated by the direct application of the covariance equation for filtered Poisson processes given by Parzen 
(1962, Section 4.5). Combining all the terms, the final expression for the autocovariance function of solution process $(6.9)$ becomes:

$\Gamma(t, t+\tau)=e^{-\alpha(2 t+\tau)} \operatorname{Var}\left(\theta_{0}-\theta_{r}\right)$

$$
+\frac{k_{c^{2} \beta^{2} e^{-\alpha \tau}}}{2 \alpha D^{2}}\left(1-e^{-2 \alpha t}\right)+\frac{\nu E\left[U^{2}\right] e^{-\alpha \tau}}{2 \alpha D^{2}}\left(1-e^{-2 \alpha t}\right)
$$

where the expectation, $E\left[U^{2}\right]$, is given by $\mathrm{Eq} \cdot 5.22$.

The variance of the process is obtained from Eq. 6.20 by setting $\tau=0$, resulting in

$$
\begin{aligned}
& \operatorname{Var}[\theta(t)]=\Gamma(t, t)=e^{-2 \alpha t} \operatorname{Var}\left[\theta_{0}-\theta_{r}\right]+\frac{K_{c}{ }^{2} \beta^{2}}{2 \alpha D^{2}}\left(1-e^{-2 \alpha t}\right) \\
& +\frac{\nu E\left[U^{2}\right]}{2 \alpha D^{2}}\left(1-e^{-2 \alpha t}\right)
\end{aligned}
$$

The autocorrelation function is obtained from Eqs. 6.20 and 6.21 as

$$
\operatorname{Corr}(t, t+\tau)=\frac{\Gamma(t, t+\tau)}{\Gamma(t, t)}=e^{-\alpha \tau}
$$

The corresponding expressions for the stationary case $(t+\infty)$ are, upon substituting for $\alpha$ :

$$
\begin{aligned}
& \operatorname{Var}[\theta(t)]=\frac{K_{c}^{2} \beta^{2}}{20 K_{e}}\left(\theta_{s}-\theta_{r}\right)+\frac{\nu E\left[U^{2}\right]}{20 K_{e}}\left(\theta_{s}-\theta_{r}\right) \\
& \operatorname{Cov}[\theta(t), \theta(t+\tau)]=\operatorname{Var}[\theta(t)] e^{-\alpha \tau}
\end{aligned}
$$




$$
\operatorname{Corr}[\theta(t), \theta(t+\tau)]=e^{-\tilde{u} \tau}
$$

\section{"Deficit" Case}

Given the more complex nature of the sample function (6.13), the moments of the process are better derived via differential equations satisfied by these moments; such equations can be derived with the help of the so-called Ito lemma or Ito differential rule which represents the chain rule of stochastic calculus. The rule is established by showing that if the scalar function $\phi=\phi(\theta, t)$ is bounded for finite $t$ and possesses continuous first partial derivatives with respect to $t$ and continuous second partial derivatives with respect to $\theta$, then, with probability one, the process $\left\{\phi(\theta, t), t>t_{0}\right\}$ satisfies the equation:

$$
\begin{aligned}
& d \phi=\left[\frac{\partial \phi}{\partial t}+a \frac{\partial \phi}{\partial \theta}+\frac{1}{2} b^{2} \frac{\partial^{2} \phi}{\partial \theta^{2}}\right] d t+\frac{b^{\partial \phi}}{\partial \theta} d W_{t} \\
& +\int_{U}[\phi(\theta+c, t)-\phi(\theta, t)] M(d t, d u)
\end{aligned}
$$

where the process $\theta_{t}$ is defined by Eq. 6.2 (see e.g. Synder, 1975). By taking expectations of both sides of (6.26), one obtains:

$$
\left.\frac{d E[\phi]}{d t}=E\left[\frac{\partial \phi}{\partial t}+\frac{\partial \phi}{\partial \theta}+0.5 b^{2} \frac{\partial^{2} \phi}{\partial \theta^{2}}\right]+\gamma E(\Delta \phi)\right]
$$

where $\Delta \phi=\phi(\theta+c, t)-\phi(\theta, t)$.

To obtain moment equations for $E\left[\theta^{k}\right]$, one chooses $\phi(\theta, t)={ }_{\theta} k, k=1,2, \ldots$ so that Eq. (48) finally becomes: 
The mean or first moment $(k=1)$ is, thus, given by the moment equation:

$$
\frac{d \bar{\theta}}{d t}=E[a]+v E[c]
$$

where $a$ and $c$ are as given in Eq. 6.2 and in which we have written $E\left[\theta_{t}\right]=\vec{\theta}$ for convenience. Upon substitution of $a$ and $c$, one gets:

$$
\frac{d \bar{\theta}}{d t}=a_{0}+a_{1} \bar{\theta}+v c_{0} E[U]
$$

or, with $\mathrm{a}_{1}=-\delta$, one obtains:

$$
\frac{d \bar{\theta}}{d t}+\delta \bar{\theta}=a_{0}+v C_{0} E[U]
$$

Eq. 6.31 is a first-order ordinary differential equation whose solution is given by:

$$
\bar{\theta}_{t}=\theta_{0} e^{-\delta t}+\frac{Q_{1}}{\delta}\left(1-e^{-\delta t}\right)
$$

where $Q_{1}=a_{0}+v C_{0} E[U]$, with $E[U]$ given by $E q$. 5.21. Upon substitution of $a_{0}$ and $c_{0},(6.32)$ becomes: 


$$
E\left[\theta_{t}\right]=\theta_{w p}+\left(E\left[\theta_{0}\right]-\theta_{w p}\right) e^{-\delta t}+\frac{\nu E[U]}{\delta D}\left(1-e^{-\delta t}\right)
$$

The variance of the process $(6.13)$ is determined from the definition:

$$
\operatorname{Var}\left[\theta_{t}\right]=E\left[\theta_{t}^{2}\right]-\left(E\left[\theta_{t}\right]\right)^{2}
$$

which, therefore, requires that we establish the equation for the second moment $(k=2)$. With $k=2$ in Eq. 6.28 , one gets:

$$
\frac{d \bar{\theta}^{2}}{d t}=E\left[2 a \theta+b^{2}\right]+\nu E\left[2 c \theta+c^{2}\right]
$$

where $\vec{\theta}^{2}=E\left[\theta_{t}^{2}\right]$, and $a, b, c$ are as given in Eq. 6.2.

After substituting for $a$ and $c$ and a little algebraic manipulation, one obtains:

$$
\frac{d \theta^{-2}}{d t}=\left(2 a_{1}+b_{1}^{2}\right) \bar{\theta}^{2}+2\left(a_{0}+\nu c_{0} E[U]\right) \bar{\theta}+2 b_{0} b_{1} \bar{\theta}+v c_{0}^{2} E\left[u^{2}\right]
$$

By replacing the second right-hand-side bracketed term with the left-hand-side term of Eq. 6.31 and rearranging, one obtains, with $a_{1}=-\delta:$ 


$$
\frac{d \bar{\theta}^{2}}{d t}-2 \bar{\theta} \frac{d \bar{\theta}}{d t}=-2 \delta \bar{\theta}^{2}+b_{1}^{2} \bar{\theta}^{2}+2 \delta(\bar{\theta})^{2}+2 b_{0} b_{1} \bar{\theta}+v c_{0}^{2} E\left[u^{2}\right]
$$

With the definition (6.34), this equation $c$ an be re-written as:

$$
\frac{d}{d t} \operatorname{Var}\left[\theta_{t}\right]+\left(2 \delta-b_{1}^{2}\right) \operatorname{Var}\left(\theta_{t}\right)=\left(b_{1}{ }^{2}+2 b_{0} b_{1}\right) E\left[\theta_{t}\right]+v c_{0}^{2} E\left[u^{2}\right]
$$

The solution of Eq. 6.38 gives the variance of the process:

$$
\operatorname{Var}\left[\theta_{t}\right]=\operatorname{Var}\left[\theta_{0}\right] e^{-\left(2 \delta-b_{1}^{2}\right) t}+\frac{Q_{2}}{2 \delta-b_{1}{ }^{2}}\left[1-\exp \left(-2 \delta t+b_{1}{ }^{2} t\right)\right]
$$

where $a_{2}=\left(b_{1}^{2}+2 b_{0} b_{1}\right) E\left[\theta_{t}\right]+v c_{0}^{2} E\left[U^{2}\right]$

in which the expectation $E\left[U^{2}\right]$ is given by Eq. 5.22. Substituting for $b_{0}, b_{1}$ and $c_{0}$, one gets:

$$
\operatorname{Var}\left[\theta_{t}\right]=\left(\operatorname{Var}\left[\theta_{0}\right] e^{-\gamma^{2} \beta^{2} t}\right) e^{-2 \delta t}+\frac{Q_{2}}{2 \delta-\gamma^{2} B^{2}}\left[1-\exp \left(-2 \delta t+\gamma^{2} \beta^{2} t\right)\right]
$$

where $Q_{2}=\gamma^{2} \beta^{2}\left(1-2 \theta_{w p}\right)\left\{\theta_{w p}+\left[E\left(\theta_{0}-\theta_{w p}\right)\right] e^{-\delta t}+\frac{\nu E[U]}{\delta D}\left(1-e^{-\delta t)}\right\}+v c_{0} E\left[U^{2}\right]\right.$

The autocovariance function of the process (6.13) is also determined from the Ito differential rule in conjunction with the definition: 
$\operatorname{Cov}[\theta(u), \theta(t)]=E\{[\hat{\theta}(u)-\bar{\partial}(u)][\theta(t)-\bar{\theta}(t)]\}$

where $0<u<t=u+\tau$ and $u$ is some fixed time. In order to use Ito's lemma, one chooses, in this case, $\phi=\left(\theta_{u}-\bar{\theta}_{u}\right)\left(\theta_{t}-\bar{\theta}_{t}\right)$ so that Eq. 6.27 becomes:

$$
\frac{d}{d t} E\left[\left(\theta_{u}-\bar{\theta}_{u}\right)\left(\theta_{t}-\bar{\theta}_{t}\right)\right]=E\left[a\left(\theta_{u}-\bar{\theta}_{u}\right)\right]+v E\left[c_{0} U\left(\theta_{u}-\bar{\theta}_{u}\right)\right]
$$

Upon substitution of a and after some lengthy algebraic manipulation, Eq. 6.42 may be written as:

$$
\frac{d}{d t} \operatorname{Cov}\left[\theta_{u}, \theta_{t}\right]=-\delta \operatorname{Cov}\left[\theta_{u}, \theta_{t}\right]
$$

The solution of this equation is:

$$
\operatorname{Cov}\left[\theta_{u}, \theta_{t}\right]=\operatorname{Var}\left[\theta_{u}\right] e^{-\delta(t-u)}
$$

which may also be written as:

$$
\operatorname{Cov}\left[\theta_{u}, \theta_{u+\tau}\right]=\operatorname{Var}\left[\theta_{u}\right] \mathrm{e}^{-\delta \tau}
$$

where $\operatorname{Var}\left[\theta_{u}\right]$ is given by Eq. 6.40 .

Once again, the autocorrelation function is simply determined as the quotient of the autocovariance and variance functions, that is: 


$$
\operatorname{Corr}[\theta(u), \theta(u+\tau)]=e^{-\delta(t-u)}=e^{-\delta \tau}
$$

For the stationary case, the mean, variance, autovariance and autocorrelation functions are, after substitution for $\delta$, respectively given by:

$$
\begin{aligned}
& E[\theta(t)]=\theta_{w p}+\frac{\nu E[U]\left(\theta_{r}-\theta_{w p}\right)}{K_{C} \bar{\varepsilon}} \\
& \operatorname{Var}[\theta(t)]=\frac{K_{C}{ }^{2} \beta^{2}\left(1-2 \theta_{w p}\right)}{2 D\left(\theta_{r}-\theta_{w p}\right) K_{C} \bar{\varepsilon}-K_{C}{ }^{2}{ }^{2}}\left\{\theta_{w p}+\frac{\nu E[U]\left(\theta_{r}-\theta_{w p}\right)}{K_{C} \bar{\varepsilon}}\right\} \\
& +\frac{\nu E\left[U^{2}\right]\left(\theta_{r}-\theta_{w p}\right)^{2}}{2 D\left(\theta_{r}-\theta_{w p}\right) K_{C} \bar{\varepsilon}-K_{C}{ }^{2}{ }^{2}} \\
& \operatorname{Cov}[\theta(u), \theta(u+\tau)]=\operatorname{Var}[\theta(u)] e^{-\delta \tau} \\
& \operatorname{Corr}[\theta(u), \theta(u+\tau)]=e^{-\delta \tau}
\end{aligned}
$$


CHAPTER VII

EVALUATION OF THE STOCHASTIC MODEL OF SOIL MOISTURE

INTRODUCTION

A stochastic model of soil moisture dynamics has been developed in the form of a pair of stochastic differential equations (SDE's) of the Ito type. The sources of stochasticity in moisture dynamics have been linked to the stochastic driving inputs of infiltration (directly related to a Poisson-process description of rainfall events) and the energy-related evapotranspiration (ET).

The nature of the SDE's are such that they lead to sample functions involving Ito integral terms which can only be computed by numerical integration. However, analytical expressions have been obtained for the moments, in particular the mean, variance and covariance.

Unfortunately, the models cannot presently be adequately tested against field conditions with the ET stochastic model adopted in this study because of the lack of knowledge of the parameter $\beta^{2}$. By making the assumption that ET is a deterministic process, however, theresulting models become amenable to field-test conditions.

Referring to Eqs. 6.16, 6.23, 6.24 and 6.25, which all represent the stationary moments for the "surplus" case, then the assumption that ET is deterministic (which implies that $\beta^{2}=0$ ), the substitution for $\alpha$, 
and writing $\varepsilon=E T p$, all lead to the following expressions for mean, covariance, variance and correlation, respectively:

$$
\begin{aligned}
& E[\theta(t)]=\theta_{r}-\frac{E\left[K_{c} E T_{p}\right]}{K_{e}}\left(\theta_{S}-\theta_{r}\right)+\frac{\nu E[U]\left(\theta_{S}-\theta_{r}\right)}{K_{e}} \\
& \operatorname{Cov}[\theta(t),(t+\tau)]=\operatorname{Var}[\theta(t)] \exp \left[\frac{-K_{e}}{D\left(\theta_{S}-\theta_{r}\right)} \tau\right] \\
& \operatorname{Var}[\theta(t)]=\frac{\nu E\left[U^{2}\right]\left(\theta_{S}-\theta_{r}\right)}{2 D K_{e}} \\
& \rho_{\theta(t)}(\tau)=\operatorname{Corr}[\theta(t), \theta(t+\tau)]=\exp \left[\frac{-K_{e}}{D\left(\theta_{s}{ }^{-\theta_{r}}\right)} \tau\right]
\end{aligned}
$$

Corresponding equations for the "deficit" case, after substituting for $\delta$, become:

$$
\begin{aligned}
& E[\theta(t)]=\theta_{w p}+\frac{\nu E[U]\left(\theta_{r}-\theta_{w p}\right)}{K_{C} E T_{p}} \\
& \operatorname{Cov}[\theta(t), \theta(t+\tau)]=\operatorname{Var}[\theta(t)] \exp \left[-\frac{K_{c} E T_{p}}{D\left(\theta_{r}-\theta_{w p}\right)} \tau\right] \\
& \operatorname{Var}[\theta(t)]=\frac{\nu E\left[U^{2}\right]\left(\theta_{r}-\theta_{w p}\right)}{2 D K_{C} E T_{p}}
\end{aligned}
$$




$$
\rho_{\theta(t)}(\tau)=\operatorname{Corr}[\theta(t), \theta(t+\tau)]=\exp \left[-\frac{K_{C} E T_{p}}{D\left(\theta_{r}-\theta_{w p}\right)} \tau\right]
$$

Koch and Mtundu (1986a), proceeding from similar assumptions about the ET process, derived exactly the same moment expressions. A different approach was used in arriving at the sample functions from which the moments were derived. The method involved first solving the linearized ODE (Eq. 5.9) for both the "surplus" and "deficit" cases under the assumption that the redistribution process occurred instantaneously. The solutions were obtained in the form of moisture depletion processes occurring between the arrival times of the infiltration events. By recursively advancing these solutions through a random series of infiltration events, which were assumed to follow a Poisson counting process, the filtered Poisson process resulted for each of the "surplus" and "deficit" cases.

Furthermore, an evaluation of the stochastic model of soil moisture (Koch and Mtundu, 1986b) was possible under the assumption of a deterministic process wich led to a re-examination of the assumptions made in the model evaluation. These assumptions are now evaluated in the following section.

\section{MAJOR ASSUMPTIONS}

A complete model of soil moisture dynamics within the root zone must deal with the processes of wetting, redistribution, drainage and ET as spatially and temporally variable phenomena. Typical approaches 
apply either Richards' equation (e.g. Philip, 1969) describing unsaturated flow or a two-phase approach which accounts for the effects of air (e.g. Morel-Seytoux, 1976). The soil water dynamic model used herein represents a simplification of these approaches. In particular, the approximation of a sharp wetting front is applied along with simplified representations of drainage and ET. Thus, the first assumption to be addressed is the suitability of this simplified model to represent the actual processes. The suitability has already been demonstrated by Koch et al (1986) and is summarized in Figs. 10 and 11. In order to achieve analytical expressions for the stochastic model, further approximations were required. First, a major assumption used in deriving the stochastic model is the independence of cumulative infiltration from a given storm on the initial soil water content at the beginning of the storm. In addition, the assumption is made that an effective value of initial soil moisture, such as the mean, can be selected for evaluation of the moments of cumulative infiltration. A further assumption is that soil moisture reacts instantaneously to an infiltration event producing a uniform soil water content in the root zone. As a result, both the wetting and redistribution phases are ignored in the stochastic model. The implication here, then, is that these phases are of very short durations relative to the interstorm depletion periods during which drainage and/or ET occur.

Another assumption that must be evaluated is the actual occurrence of the wet (or "surplus") and dry (or "deficit") states within the soil. Since the stochastic model is only developed for the two limiting cases, its applicability is restricted to situations where 
either the "surplus" or "deficit" cases persist. Simulation results for two climates and three soil types will show how reasonable the assumption of limiting behavior is and in what hydrologic environments one might expect to observe such behavior. Following an evaluation of the stochastic properties of cumulative infiltration, a systematic examination of all the above assumptions is presented to assess the conditions under which the stochastic model is applicable.

\section{BEHAVIOR OF THE INFILTRATION PROCESS}

Cumulative infiltration represents the stochastic input into the unsaturated soil moisture system and primarily dictates the occurrence of excess precipitation leading to the process of surface runoff. Given any storm event, the amount of water that infiltrates through the soil surface is a function of the properties of the soil as well as the characteristics of the storm such as its intensity and duration. In a given interval $[0, t)$, the cumulative infiltration also depends on the interarrival times of storms inasmuch as the dynamics of the soil moisture depletion process, driven by the forces of gravity, capillarity and evapotranspiration, dictate the initial soil water content at the beginning of each storm event. A model of infiltration and moments of cumulative infiltration have already been presented in Chapter $V$.

With the analytical form of the moment equation for cumulative infiltration, it is possible to numerically evaluate the behavior of the infilration process and, in particular, the dependence of the moments on initial soil moisture. For mathematical tractability, the dependence was ignored in the stochastic model of soil moisture; this effect can 
now be evaluated.

The evaluation of the infiltration model was performed on the basis of the same three soil types and two different climatic regimes considered in Chapter VI. Properties of the soils used in the evaluation are given in Table II and represent a broad range of soil types whereas the storm parameters are presented in Table I.

Sensitivity of the first two moments of cumulative infiltration to various soil-types and antecedent moisture conditions is illustrated for the Blacksburg climate in Figures 18 and 19. The abscissa in these figures is the initial moisture normalized with respect to the residual moisture and defined as $\theta_{i}{ }^{*}=\left(\theta_{j}-\theta_{r}\right) /\left(\theta_{s}-\theta_{r}\right)$. Based on the trend of the lines, little variation is expected for drier conditions (that is, for $\theta<\theta_{r}$ ). The moments demonstrate a marked dependence on soil type, as would be expected, with higher infiltration for more permeable soils. For both the first and second moment of cumulative infiltration, sandy loam shows almost no dependence on $\theta_{j}$ while clay loam is quite variable, particularly for $\theta_{j}$ yalues near saturation. From these results, one might expect that if the probability mass of $\theta_{i}$ is concentrated near the "dry" end of the range, the moments of cumulative infiltration and thus soil water content would show little dependence on $\theta_{i}$, in general. Figure 20 compares the ratio of mean cumulative infiltration to mean storm depth $(1 / \lambda \beta)$ between the two different climatic regimes for the same soil. Evidently, this ratio varies with climate and also depends, to a significant extent, on the initial moisture conditions. However, the general behavior appears to be similar for these particular climate types. The only difference in 


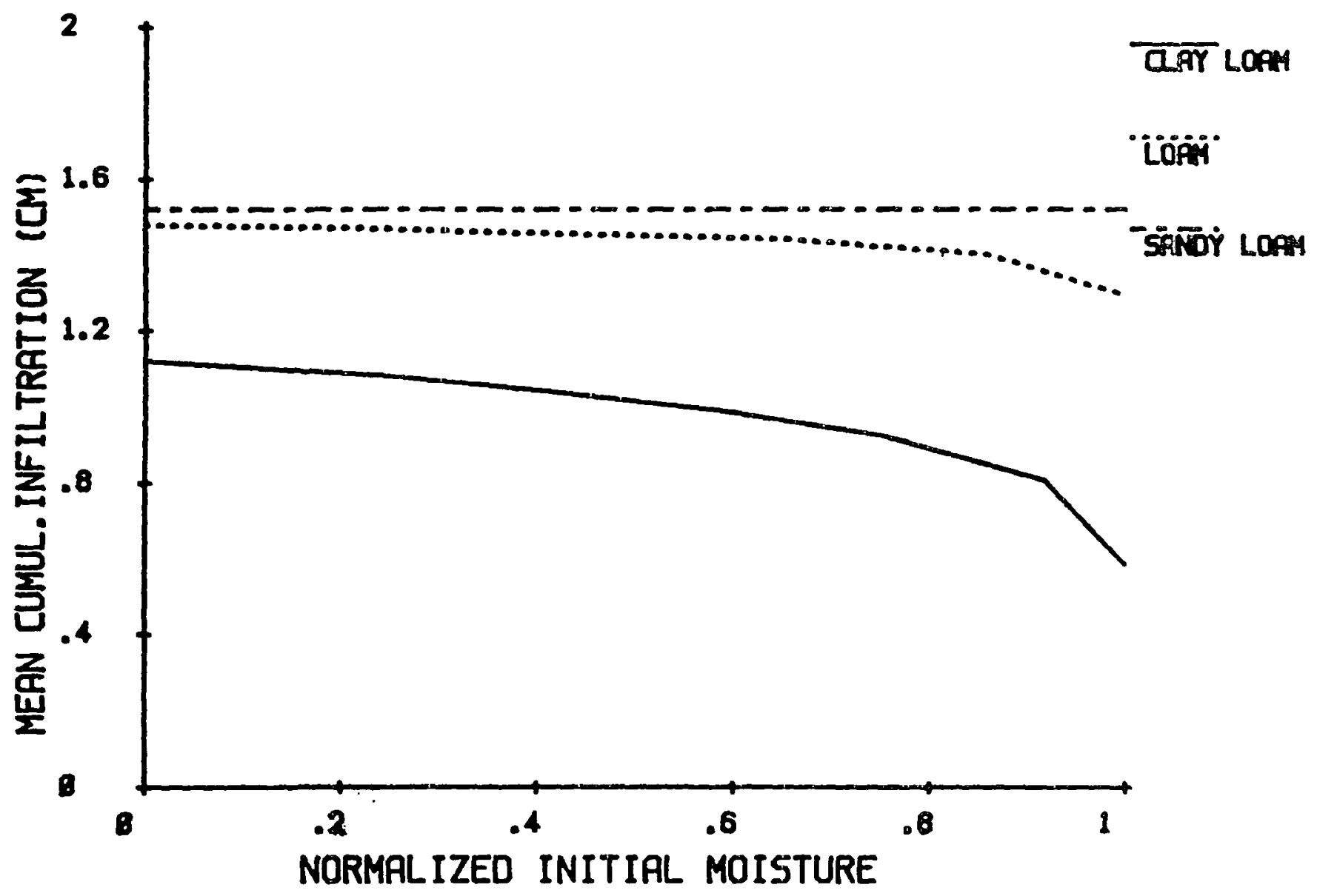

Figure 18. Mean of cumulative infiltration for different soil types; BTacksburg, Virginia climate. 


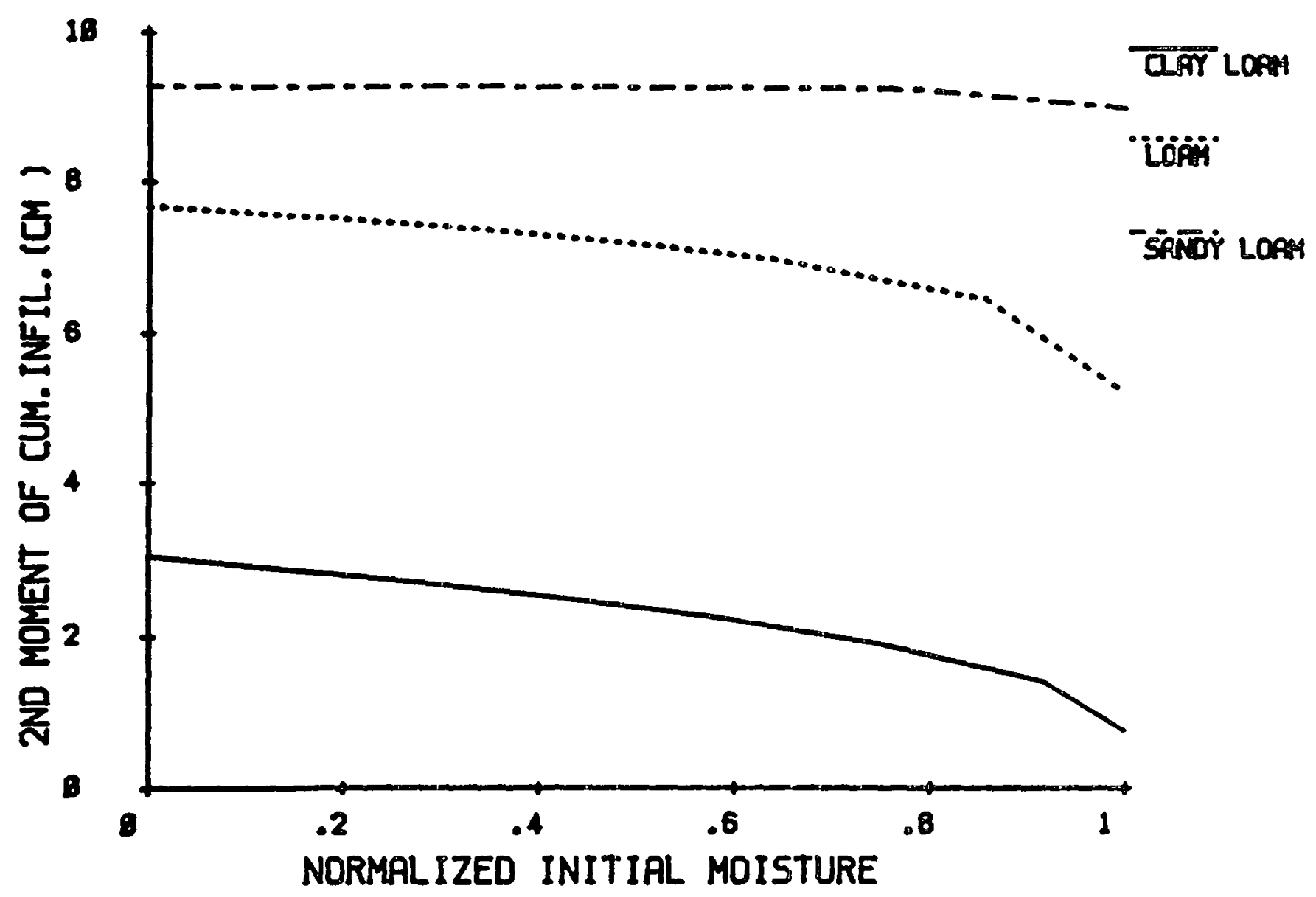

Figure 19. Second moment of cumulative infiltration for different soil types; Blacksburg, Virginia climate. 


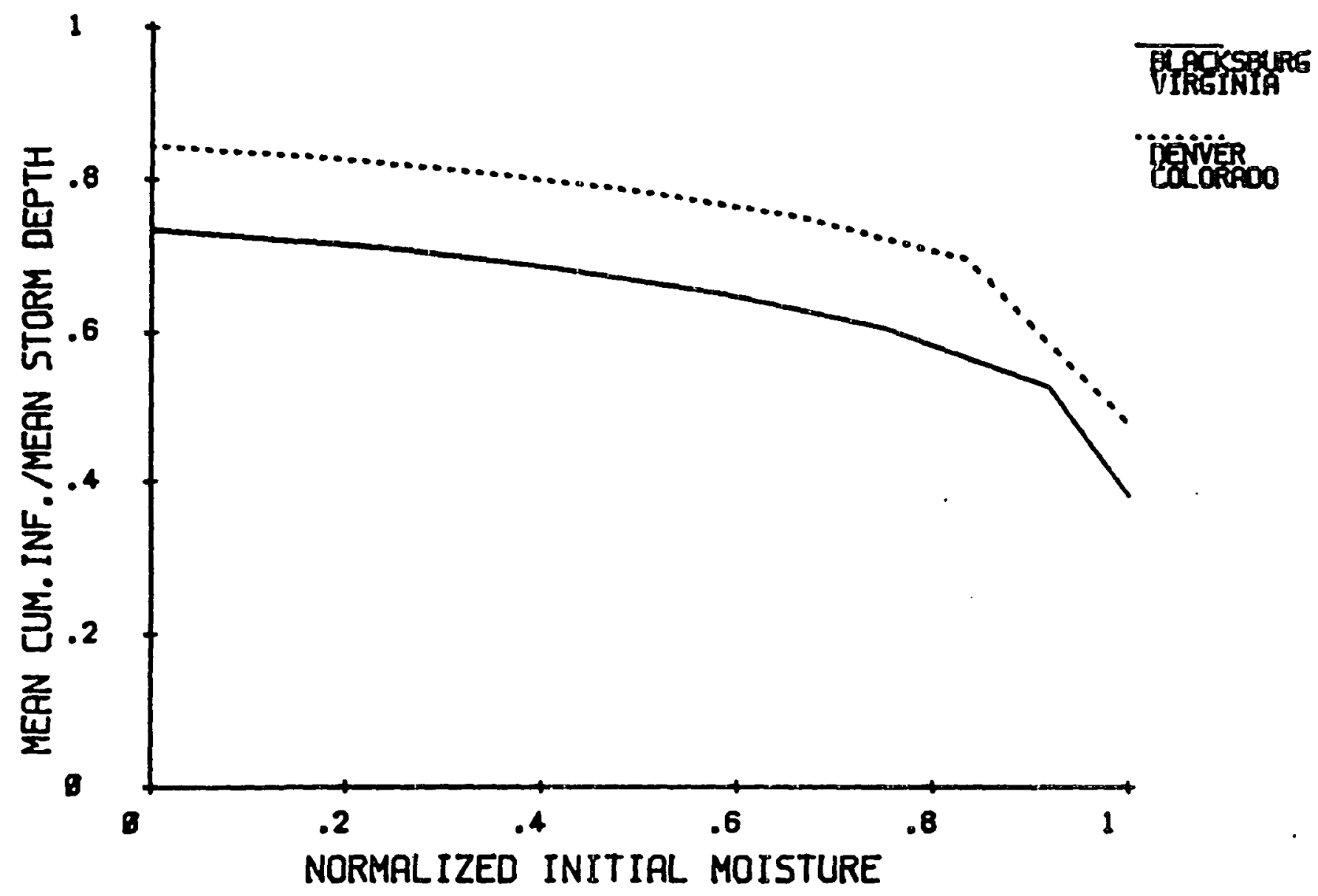

Figure 20. Mean cumulative infiltration relative to mean storm depth for different $\mathrm{climates;} \mathrm{clay}$ loam soil 
behavior is in the absolute magnitude of the ratio.

\section{OCCURRENCE OF LIMITING BEHAVIOR}

Recalling that the stochastic models have been developed only for cases where the soil moisture is in either a "surplus" or "deficit" condition, the actual occurrence of this limiting behavior is of interest. Simulations were performed for the three climatic conditions presented in Table I (Chapter VI) and for each of three soil types: clay loam, loam and sandy loam. The Blacksburg climatic data were selected to represent a "surplus" soil moisture situation as was the Denver wet season data where the storm duration is longer and ET rate lower. The Denver dry season data were selected to represent the "deficit" condition. Upon performing the simulations, it was discovered that, in certain situations, it was not possible to maintain a single condition in the soil moisture but that both the "surplus" and "deficit" states were observed. In particular, for the more permeable soils like loam, and more often sandy loam, the "surplus" situation was not maintained throughout the random interstorm period in many cases. Thus, the soil moisture occasionally passed over to the "deficit" state as a result of rapid drainage rates. This phenomenon occurred for both the Blacksburg and Denver "surplus" simulations. For the Denver dry season case, the soil moisture did persist in the "deficit" condition throughout the simulation period indicating that this limiting case is feasible for all soil types under the climatic conditions tested.

From these results, one observes that, under certain combinations of soil and climate, the "surplus" case cannot be maintained and thus 
the performance of the stochastic model might be excepted to deviate from simulation results, even if all other assumptions had no effect. For the "deficit" case and for the clay loam soils, both limiting cases appear to be feasible realizations of the natural processes under the conditions investigated.

\section{SENSITIYITY TO INITIAL SOIL MOISTURE}

A fundamental assumption made in the derivation of the stochastic model of soil moisture was to neglect the dependence of infiltration and thus soil moisture on the initial value of soil water content at the beginning of a storm event. However, it has been shown that there is, indeed, some dependence of the moments of cumulative infiltration on the initial soil moisture when $\theta_{i}$ is close to saturation and in particular for less permeable soils. Since the moments of soil moisture, as derived from the simplified stochastic model are directly dependent on the moments of cumulative infiltration, a similar dependence on $\theta_{i}$ would be expected. To evaluate this sensitivity, the mean and standard deviation of soil moisture were calculated using the analytical expressions given by Eqs. 7.1 and 7.3, parameterized by initial soil water content which appears in the moment equations for cumulative infiltration. The same three climatic conditions corresponding to the Blacksburg "surplus", Denver "surplus" and Denver "deficit" cases and three soil types were used for this demonstration.

The results of this analysis are presented in Figures 21 through 24 showing the dependence of the mean and standard deviation of soil moisture on the initial water content of the soil. For the Blacksburg 
"surplus" case, Figure 21 shows little dependence of the mean on initial moisture content (shown normalized as $\left(\theta_{j}-\theta_{r}\right) /\left(\theta_{s}-\theta_{r}\right)$, where $\theta_{r}$ is the residual water content and $\theta_{s}$ the soil moisture at natural saturation) across all three soil types, although some variation is noted as the clay loam soil reaches saturation. On the other hand, the standard deviation, Figure 22, shows considerably more sensitivity, particularly for clay loam soil where there is marked dependence throughout the range of initial water contents investigated. Considerably less variation is apparent for 10 am and there is almost no trend for the sandy loam soil. Similar behavior is noted for the Denver "surplus" case which is not shown. These results follow closely the behavior of the first and second moments of cumulative infiltration in relation to initial water content as might be expected. In the Denver "deficit" case, the mean soil moisture shows little dependence on $\theta_{j}$ as would be expected since cumulative infiltration exhibited this same behavior (see Figure 23). (In this case the initial water content is normalized as $\left(\theta_{i}-\theta_{\text {wp }}\right) /\left(\theta_{r}-\theta_{w p}\right)$, where $\theta_{\text {wp }}$ is the permanent wilting point). Again, some small variation is noted in the standard deviation for clay loam (Figure 24); otherwise, $\theta$ is apparently insensitive to $\theta_{j}$.

In general, then, the assumption of independence between $\theta$ and $\theta_{\mathbf{j}}$ is reasonable in many situations, particularly for more permeable soils in the "surplus" case. Further, the mean is less dependent on $\theta_{j}$ than is the standard deviation. In the "deficit" case, there is almost no interrelationship between moments of $\theta$ and the initial water content of the soil. The assumption of independence between hydrologic processes 


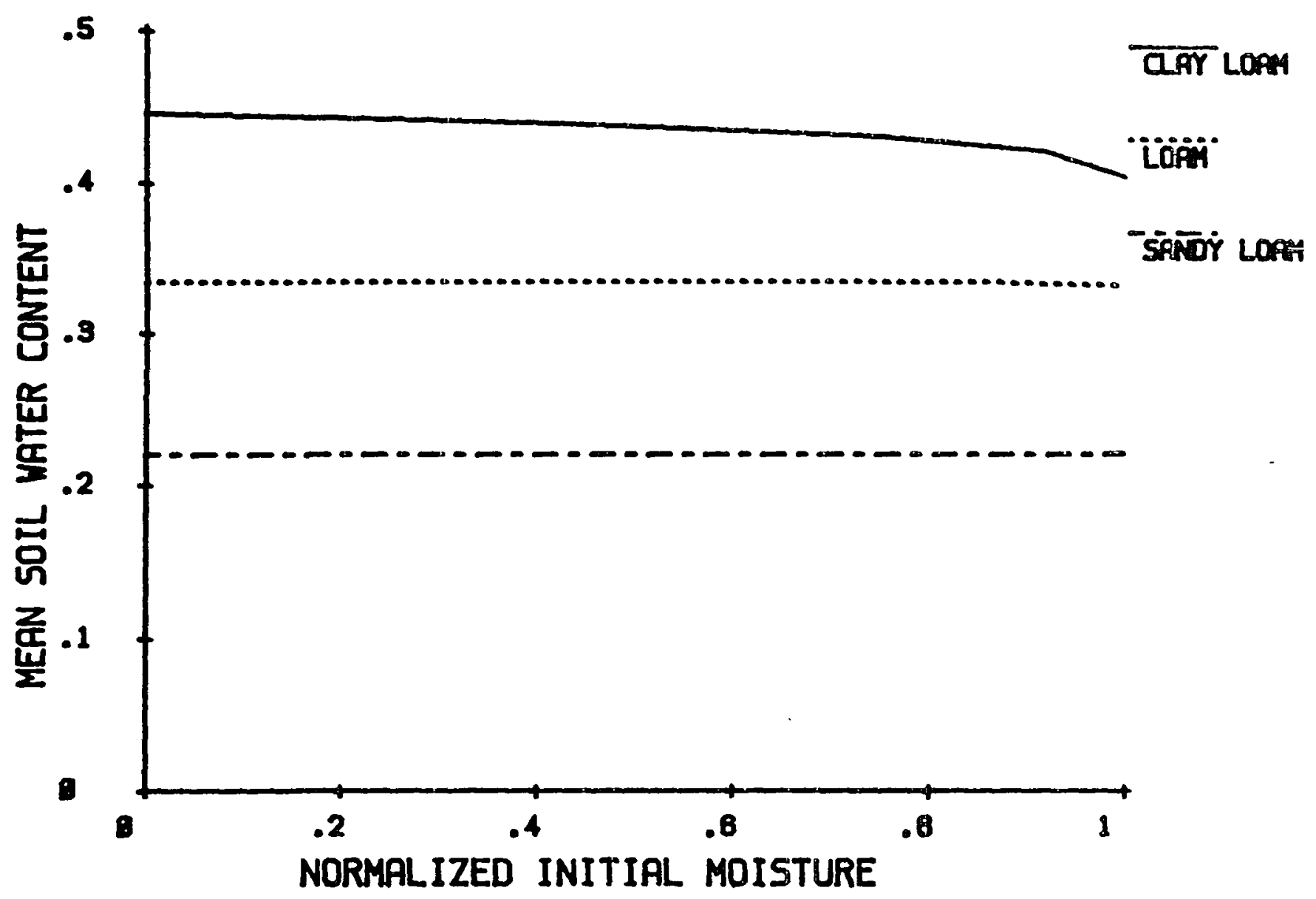

Figure 21. Expected value of soil water content for different soil types; BTacksburg, Virginia climate ("surplus" case). 


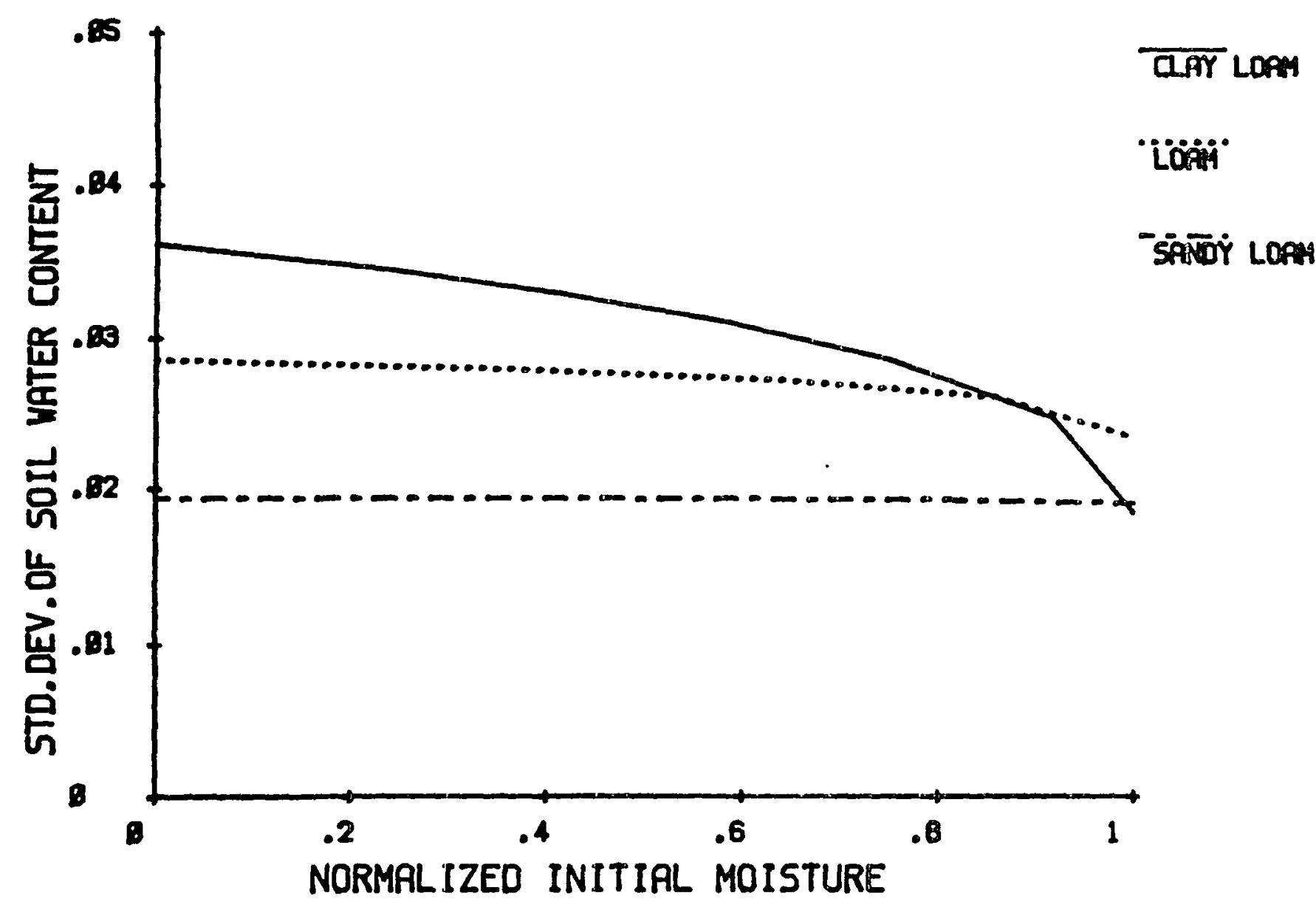

Figure 22. Standard deviation of soil water content for different soil types; Blacksburg, Virginia climate ("surplus" case). 


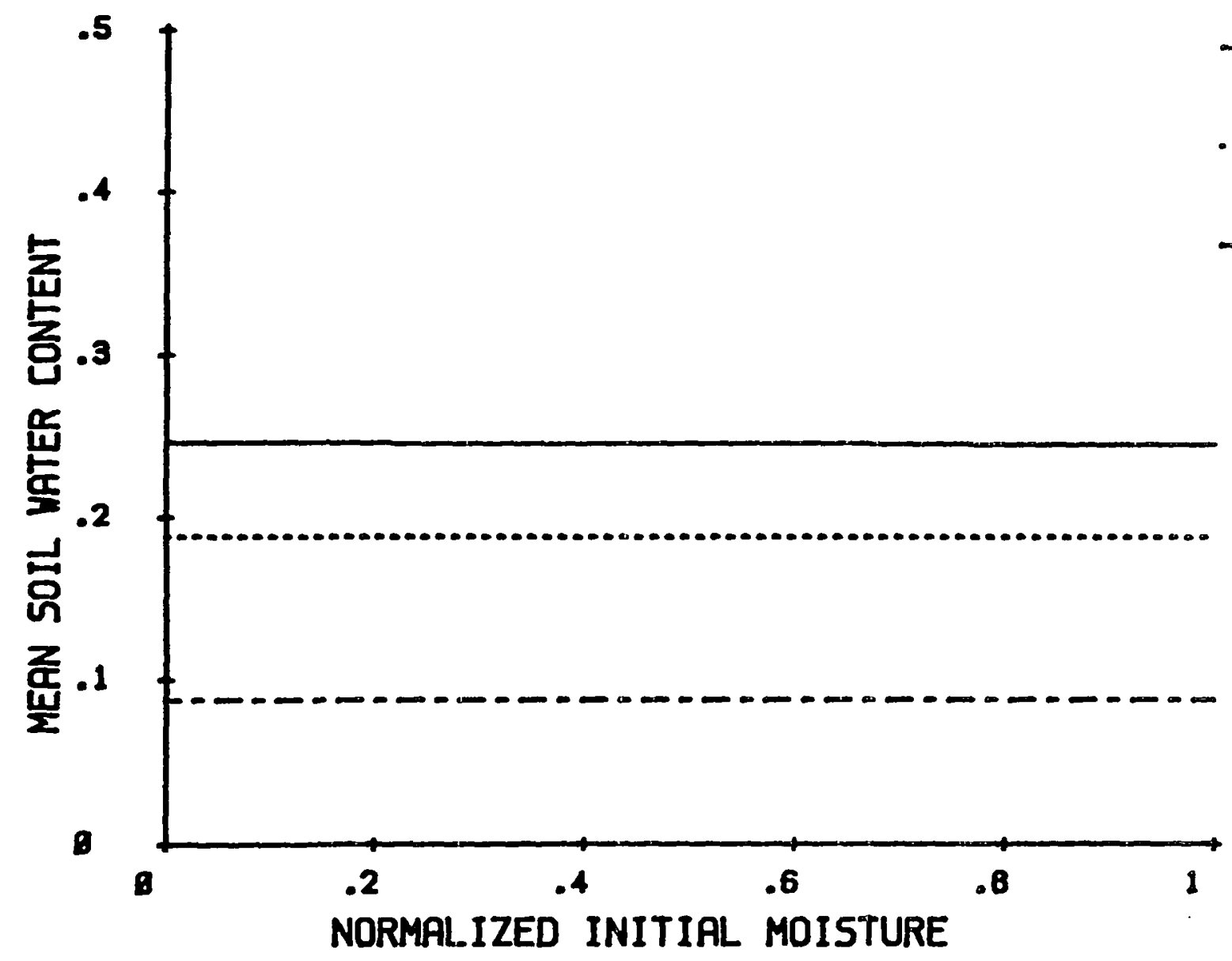

Figure 23. Expected value of soil water content for different soil types; Denver, Colorado climate ("deficit" case).

CLAT LOPA4

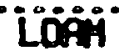

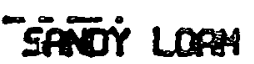




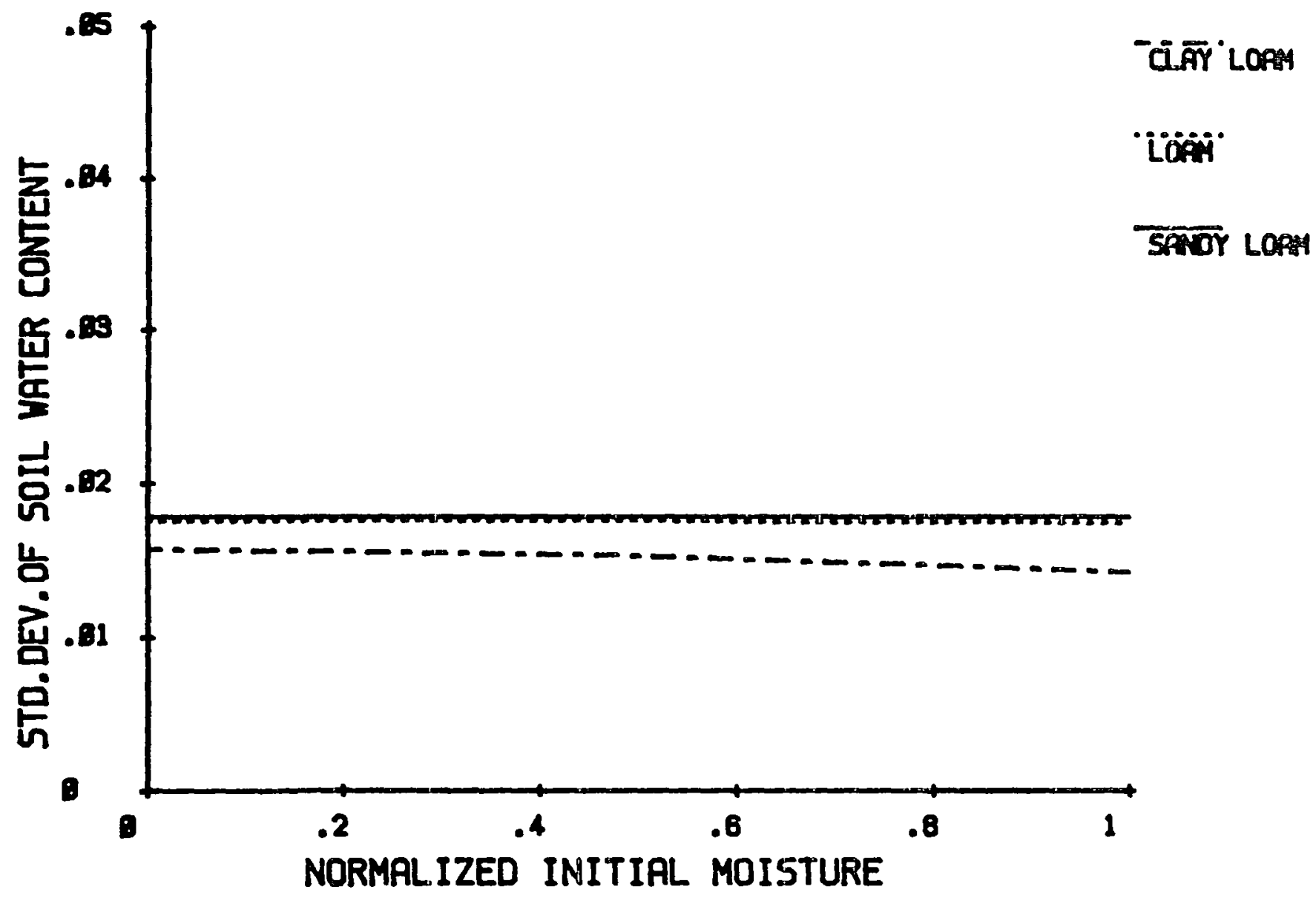

Figure 24. Standard deviation of soil water content for different soil types; Denver, Colorado cl imate ("deficit" case). 
and initial soil moisture will be examined in the following chapter in which the probability distribution of initial moisture is investigated.

\section{EFFECTS OF OTHER SIMPLIFYING ASSUMPTIONS}

Having established that the limiting cases are reasonable in certain situations and that there is some encouragement for the decoupling of $\theta$ from its dependence on $\theta_{i}$, the impacts of the remaining assumptions underlying the stochastic model are evaluated. The purpose of this part of the evaluation is to establish bounds within which the simplified stochastic model is indeed a reasonable representation of the actual process and can be eventually used in prediction. Specifically, the effect of ignoring the wetting and redistribution phases and selection of the mean of $\theta_{j}$ as the effective value for evaluation of the moments of cumulative infiltration are assessed. The same three climatic conditions and soil types are employed in the evaluation. Results of a Monte Carlo simulation of the determinstic soil moisture model (Eqs, 5.11 to 5.15) are used as the basis for comparison with the analytical moment expressions developed in the first section of this chapter. However, since the simulation model is "complete" in the number of processes it includes, two other simulation models were developed by slightly modifying the "complete" one for use in the comparison. One model assumed "instantaneous wetting" to evaluate the assumption in relation to the "complete" and simplified stochastic model. The other model assumed "instantaneous wetting and redistribution" and represents exactly the stochastic model with the exception that the initial soil moisture is variable in the 
simulation model and assumed to be constant at the mean value in the stochastic model. First, attention is focused on the mean and standard deviation. Then, an assessment of the effects of the assumptions on the prediction of time dependence in soil moisture is made.

The results of these simulations for the mean and standard deviation, in comparison to the predictions using the stochastic model, are presented in Table III. However, to illustrate the effect of the assumptions more clearly, Figures 25 through 28 have been prepared. On these figures the ordinate is the numerical value of either the mean or standard deviation of soil moisture. The abscissa represents soil type ranging from clay loam on the left to sandy loam on the right. No numerical significance should be attached to this axis and the equal spacing between soil types is selected for convenience. There are four lines on each of these figures representing results of the three simulation models ("complete", "without wetting" and "without wetting and redistribution") and the computed moment from the simplified stochastic model. In applying the analytical moment equations for soil moisture, the average value of $\theta_{\boldsymbol{j}}$ obtained from the "complete" simulation model was taken as the effective initial water content in calculating the first and second moments of cumulative infiltration. The relationship of these lines displays graphically the effect of the various assumptions.

In Figures 25 and 26, results for the Denver "surplus" case are presented. The Blacksburg "surplus" case exhibited similar behavior so the results are not shown. Referring to Fig. 25, the mean value of $\theta$, is observed to decrease as soil permeability increases and the effect of 
TABLE $X$

COMPARISON OF PHYSICALLY BASED MOMENTS OF NORMALIZED INITIAL SOIL MOISTURE WITH THOSE 8ASED ON MONTE CARLO SIMULATIONS

\begin{tabular}{|c|c|c|c|c|c|c|c|c|c|c|}
\hline \multirow[t]{2}{*}{ Location } & \multirow[t]{2}{*}{ Type of Soll } & \multicolumn{3}{|c|}{ "Complete"Model } & \multicolumn{3}{|c|}{$\begin{array}{l}\text { "Instantaneous Hetting } \\
\text { and Redistribution" Model" }\end{array}$} & \multicolumn{3}{|c|}{ Stochastic Nodel } \\
\hline & & $\mu$ & $\sigma^{2}$ & $\rho_{1}$ & $\mu$ & $\sigma^{2}$ & $\rho_{1}$ & p & $\sigma^{2}$ & $\rho_{1}$ \\
\hline $\begin{array}{l}\text { Bl acksburg } \\
\text { "Surplus" } \\
\text { Case }\end{array}$ & $\begin{array}{l}\text { Clay Loam } \\
\text { Loam } \\
\text { Sandy Loam }\end{array}$ & $\begin{array}{l}0.722 \\
0.474 \\
0.295\end{array}$ & $\begin{array}{l}0.0196 \\
0.0327 \\
0.0261\end{array}$ & $\begin{array}{r}0.444 \\
0.049 \\
-0.138\end{array}$ & $\begin{array}{l}0.422 \\
0.124 \\
0.0333\end{array}$ & $\begin{array}{l}0.0346 \\
0.0159 \\
0.00322\end{array}$ & $\begin{array}{l}0.838 \\
0.546 \\
0.266\end{array}$ & $\begin{array}{l}0.455 \\
0.141 \\
0.0378\end{array}$ & $\begin{array}{l}0.0569 \\
0.0317 \\
0.00743\end{array}$ & $\begin{array}{c}\cdots \\
0.543 \\
0.319\end{array}$ \\
\hline $\begin{array}{l}\text { Denver } \\
\text { "Deficit" } \\
\text { Case }\end{array}$ & $\begin{array}{l}\text { Clay Loam } \\
\text { Loan } \\
\text { Sandy Loam }\end{array}$ & $\begin{array}{l}0.133 \\
0.139 \\
0.140\end{array}$ & $\begin{array}{l}0.0046 \\
0.0058 \\
0.0060\end{array}$ & $\begin{array}{l}0.847 \\
0.845 \\
0.848\end{array}$ & $\begin{array}{l}0.129 \\
0.135 \\
0.135\end{array}$ & $\begin{array}{l}0.00433 \\
0.00543 \\
0.00565\end{array}$ & $\begin{array}{l}0.844 \\
0.842 \\
0.845\end{array}$ & $\begin{array}{l}0.228 \\
0.238 \\
0.239\end{array}$ & $\begin{array}{l}0.00944 \\
0.0121 \\
0.0124\end{array}$ & $\begin{array}{l}0.902 \\
0.902 \\
0.902\end{array}$ \\
\hline
\end{tabular}

$\mu \equiv$ mean, $\sigma^{2} \equiv$ variance, $\rho_{1} \equiv 1 a g-1$ autocorrelation coefficient 


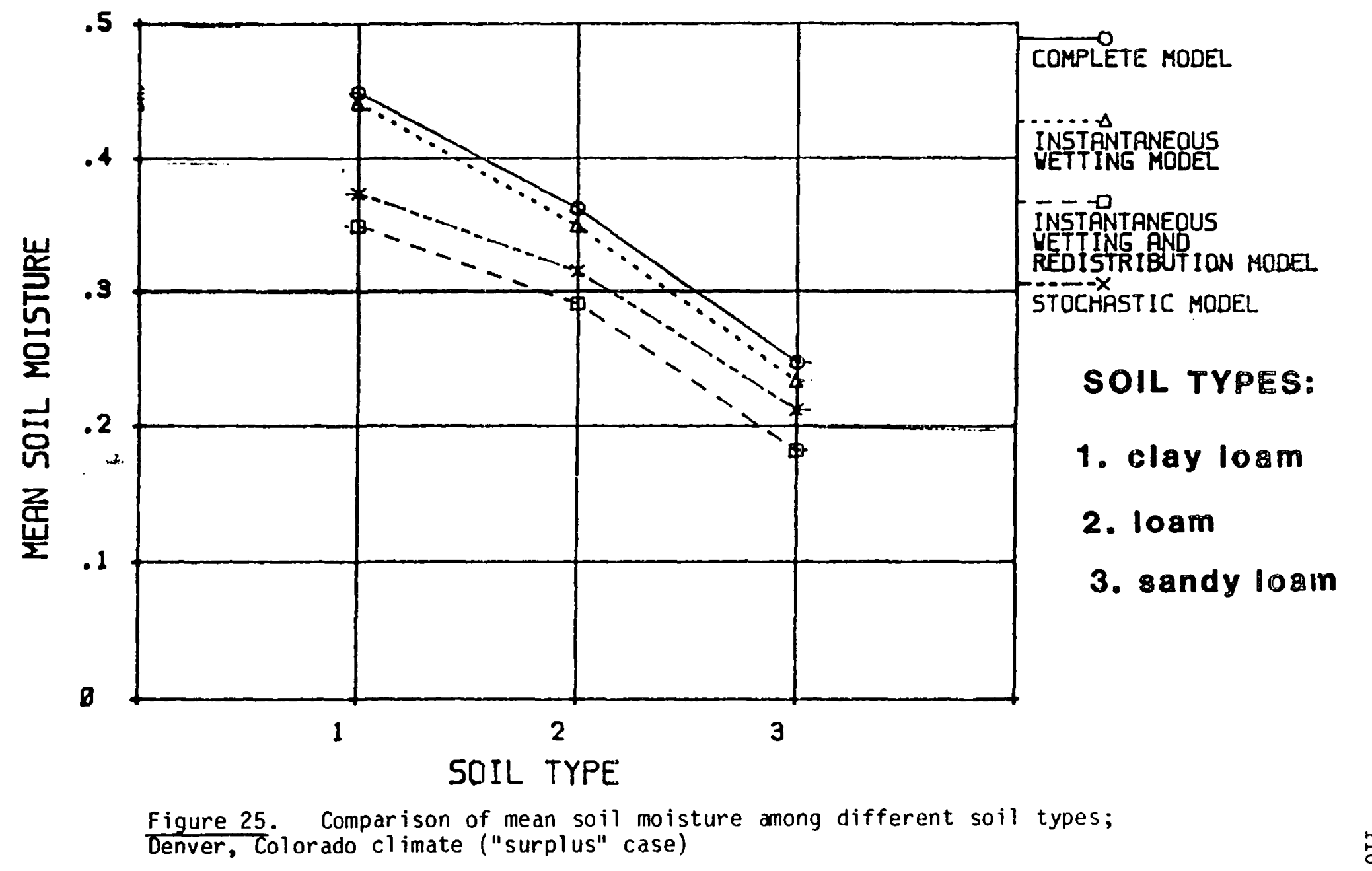


the assumptions are consistent across the soil types. The assumption of instantaneous wetting biases the estimated mean downward for all soil types. Further, the assumptions of both instantaneous wetting and redistribution has a similar but more pronounced effect providing a greater bias in the mean.

Since the simulation model with instantaneous wetting and redistribution duplicates the simplified stochastic model except that a variable $\theta_{j}$ is used in the simulation, the former model serves as a basis for evaluating the use of the mean value of initial water content as the effective value in the stochastic model. As can be seen in Figure 25 , a variable $\theta_{j}$ produces a lower mean value than a constant average $\theta_{i}$; however, the stochastic model is still biased low when compared to the complete model.

Comparison of the behavior of the simulation models with the stochastic model in terms of the standard deviation is shown in Figure 26 for the Denver "surplus" case. Evidently, the complete simulation model as well as the approximate model with instantaneous wetting behaved quite differently from the stochastic model. This behavior is primarily due to the transitory nature of the soil moisture states (passing from the "surplus" into the "deficit" condition), particularly for the more permeable soil types. Thus, the resulting range of values of $\theta$ was significantly increased with a corresponding increase in the standard deviation. Thus, for this particular climatic condition, the stochastic model does not appear appropriate for more permeable soil types. Results from the approximate simulation model with both instantaneous wetting and redistribution show a trend similar to the 


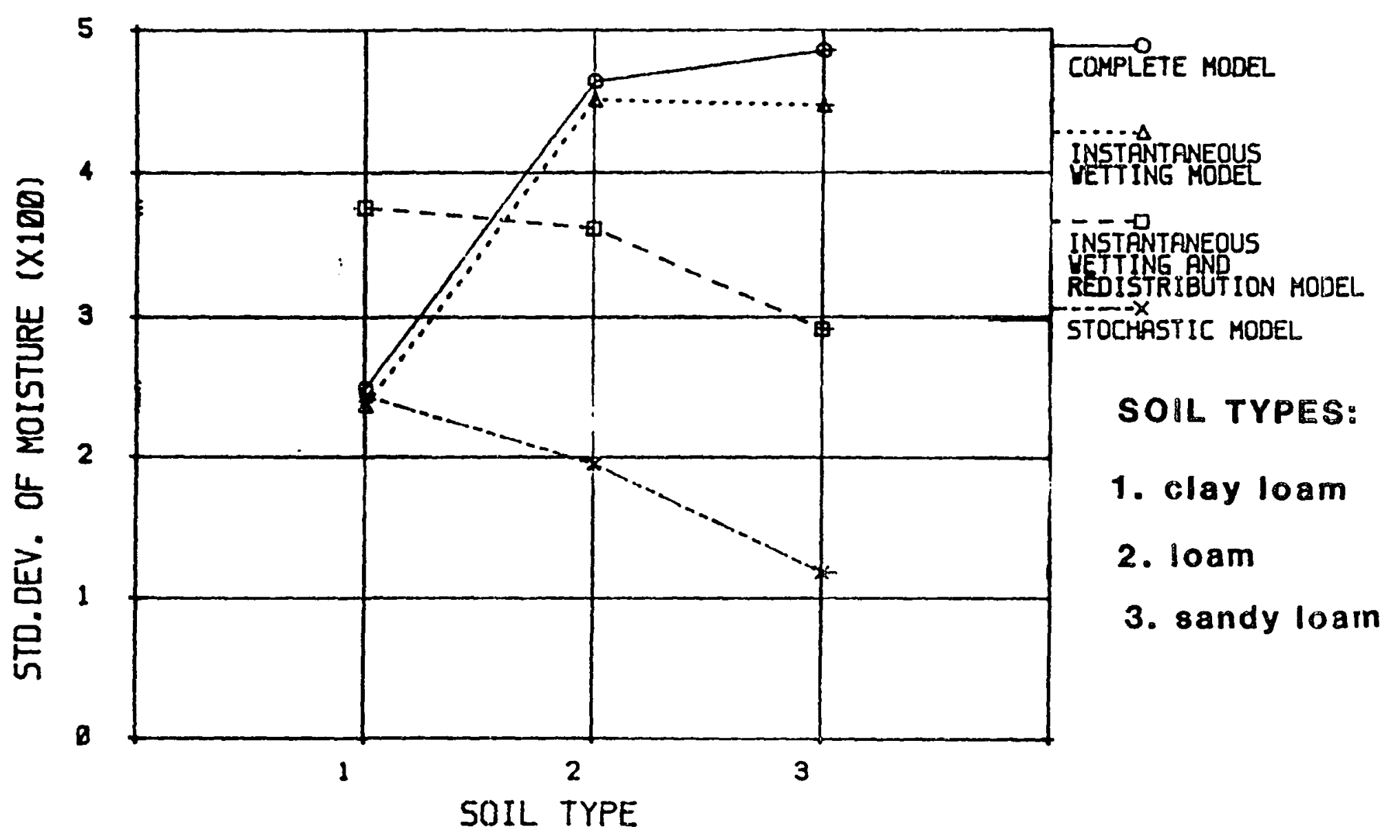

Figure 26. Comparison of soil moisture variability among different soil types; Denver, Colorado cl imate ("surplus" case) 
stochastic model but with a significantly higher standard deviation; this results from using the mean value of $\theta ;$ as the effective value in computing $E[U]$ and $E\left[U^{2}\right]$ in the stochastic model. The stochastic model significantly underestimates the variability of soil moisture for permeable soil types in this situation due both to the effect of the simplifying assumption in the soil moisture dynamics and the fact that the "surplus" case is not continuously maintained.

Turning to the Denver "deficit" case, the results are considerably more encouraging. As is apparent from figure 27, the assumptions of instantaneous wetting and redistribution have essentially no effect on the simulated mean value. However, the use of the constant mean $\theta_{j}$ in the stochastic model produces a slight but consistent over-estimation of the mean across all soil types. The standard deviation computed from the simulations behaves similarly to that of the stochastic model as shown in Figure 28. In this case, the assumption of instantaneous wetting has the effect of decreasing the estimated variability of soil moisture. However, the assumption of a constant, effective value of $\theta_{j}$, as opposed to the variable initial water content approach of the simulation models, has the most significant effect. These assumptions combine to bias the predicted standard deviation downward.

For a measure of the effects of the assumptions on the correlation structure of soil moisture as predicted by the stochastic model, the correlograms of the three simulation models have been computed and compared to the stochastic model. These results are shown in Figures 29 and 30. For the Blacksburg "surplus" case (Fig. 29), the 


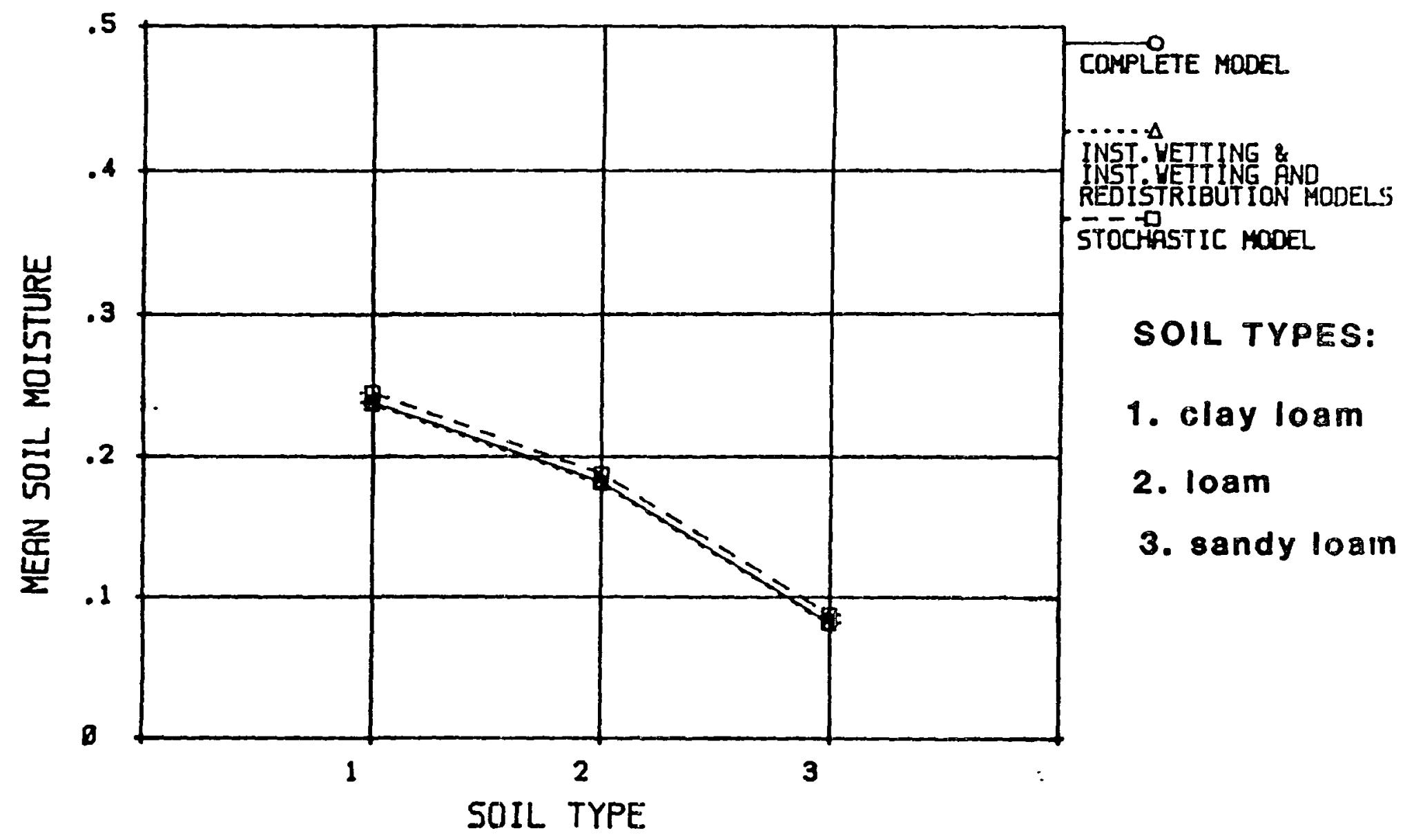

Figure 27. Comparison of mean soil moisture among different soil types; Denver, Colorado climate ("deficit" case) 


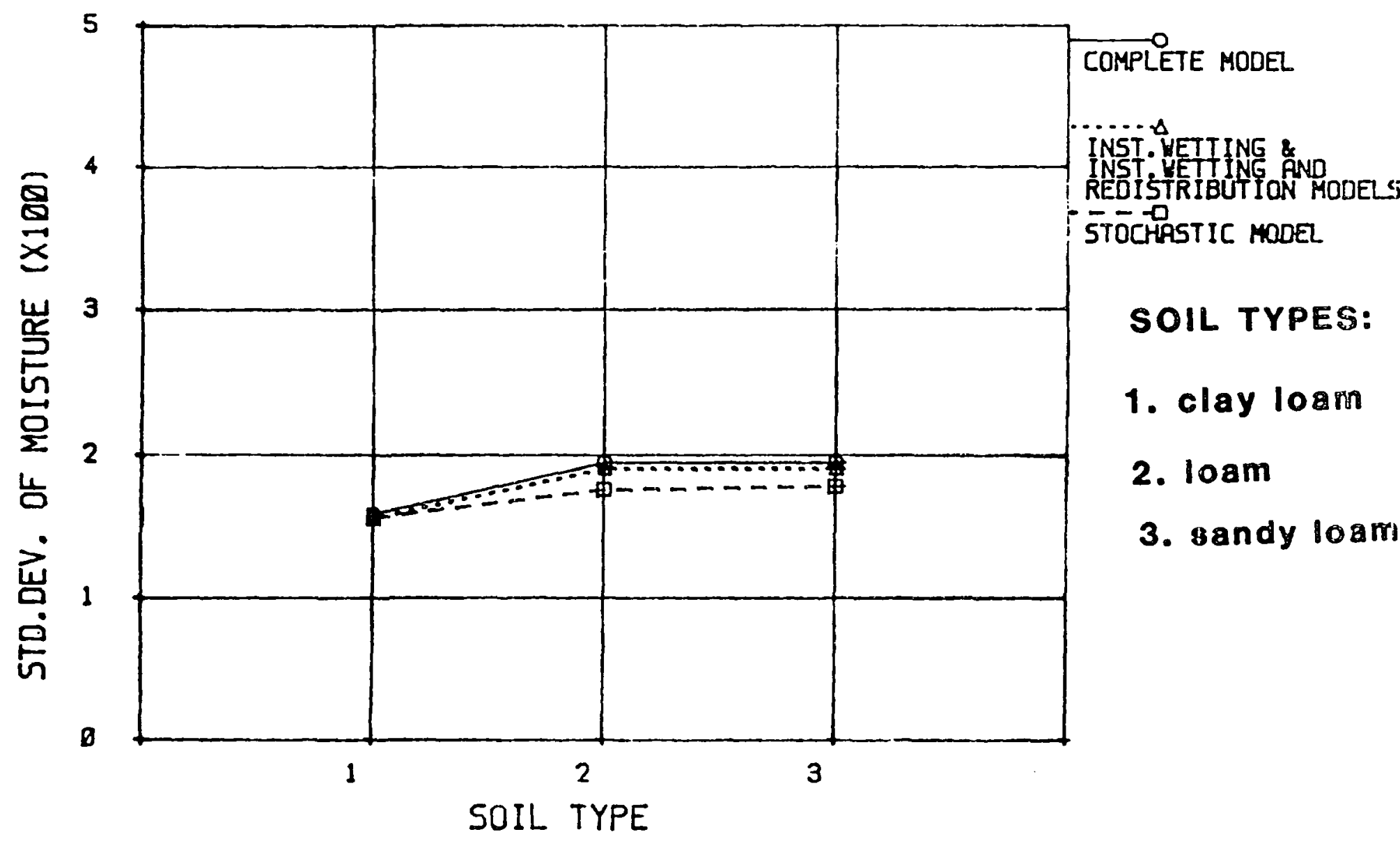

Figure 28. Comparison of soil moisture variability among different soil types; Denver, Colorado cl imate ("deficit" case) 


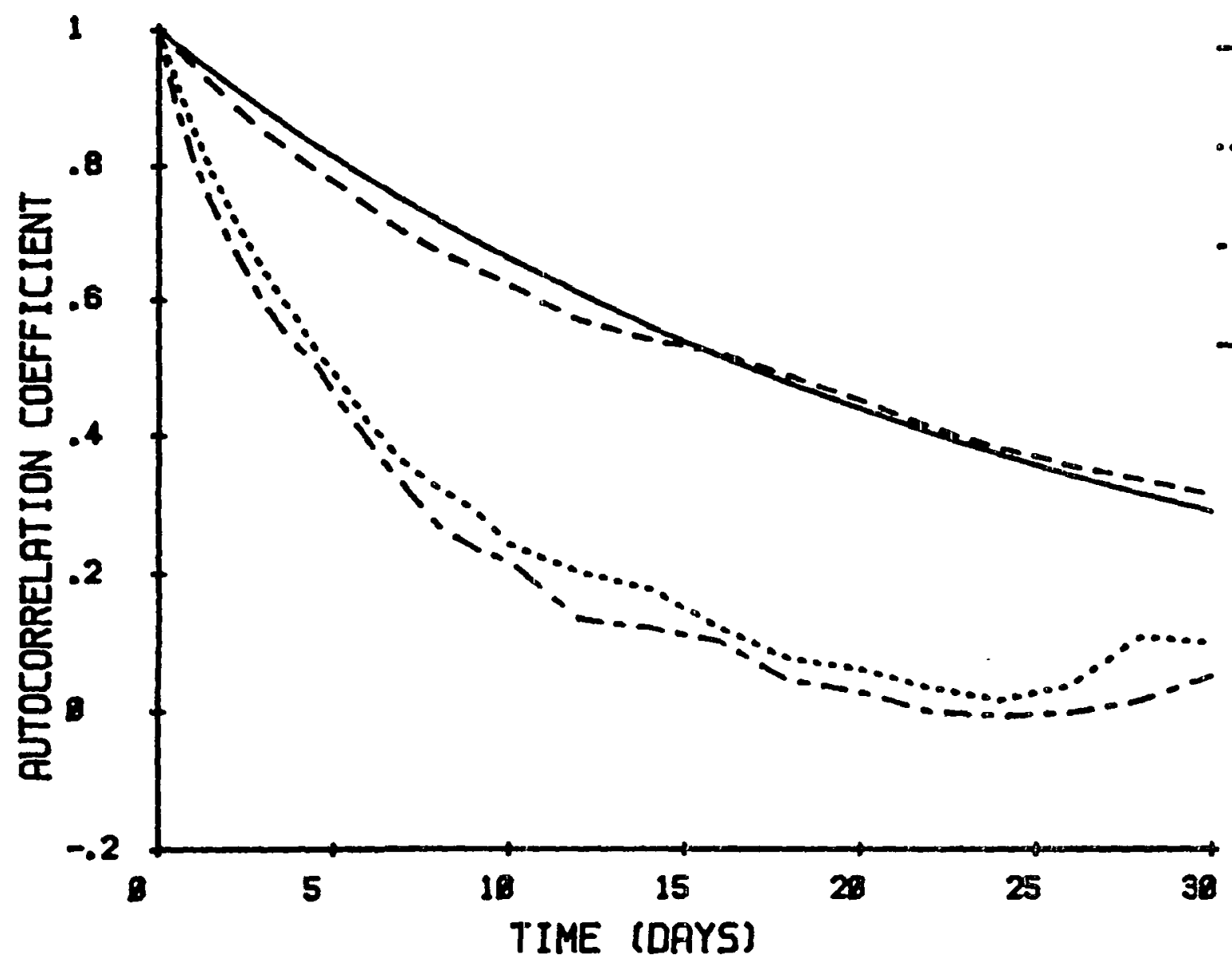

CŌTPLiete mogel

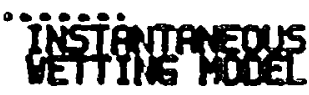

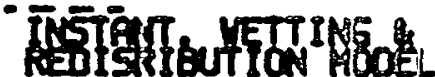

STOCHASTIC MOELL

Figure 29. Comparison of autocorrelation functions among the soil moisture

dynamic and stochastic models for the Blacksburg, Virginia climate 
actual correlation is much lower than that predicted by the stochastic model, principally due to ignoring the wetting and redistribution phases. The assumption of instantaneous wetting increases correlation to some extent but the redistribution accounts for most of the difference between the simulation and simplified stochastic models. Similar results are obtained for the Denver "surplus" case as shown in Figure 30 .

In the Denver "deficit" case (Fig. 31) reasonable agreement is demonstrated between the simulation model and the stochastic model. In this case the assumptions of instantaneous wetting and redistribution did not significantly affect the high time dependence predicted by the simplified stochastic model. 


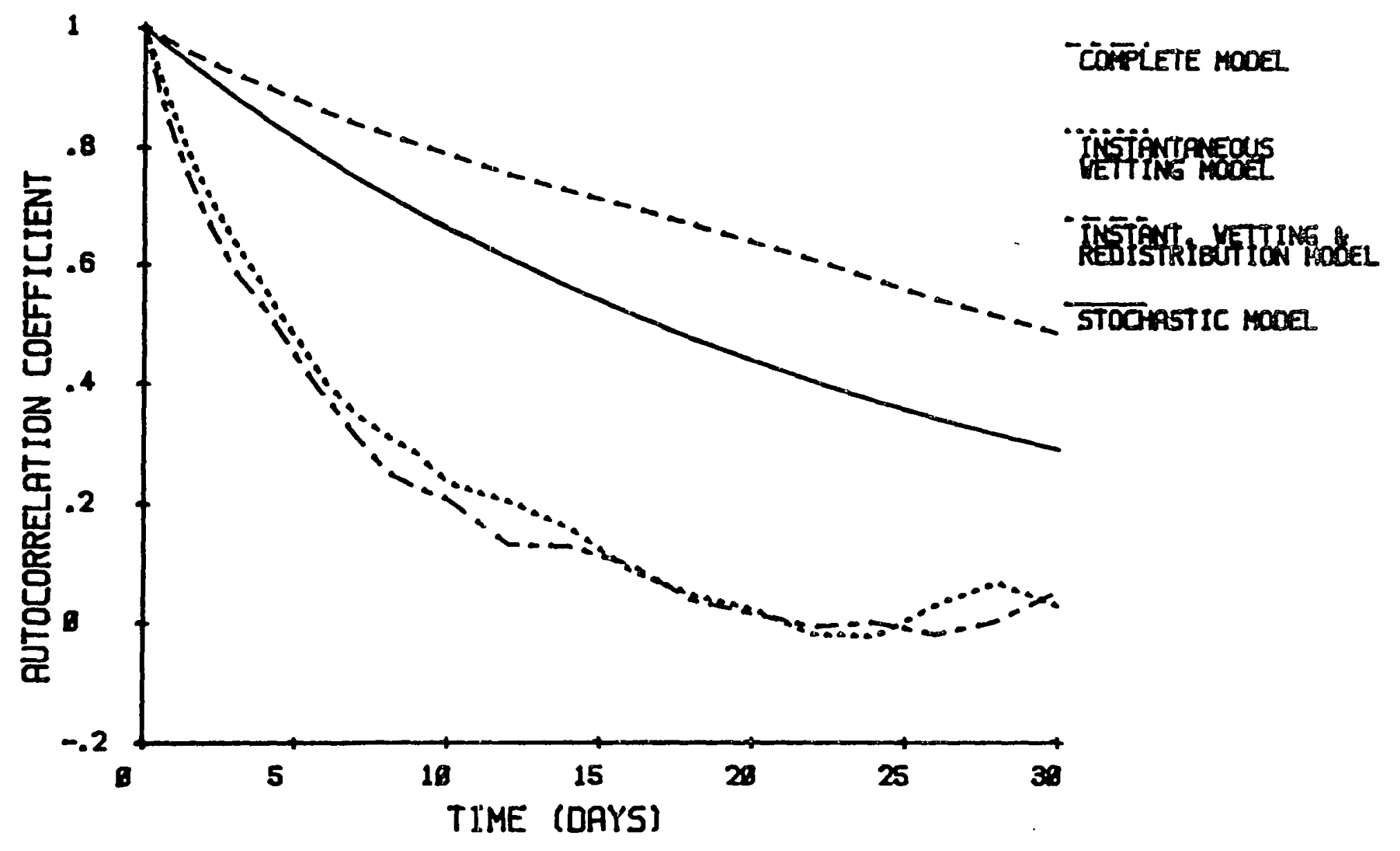

Figure 30. Comparison of autocorrelation functions among the soil moisture dynamic and stochastic models for the Denver, Colorado climate

("surplus" case); clay loam soil. 


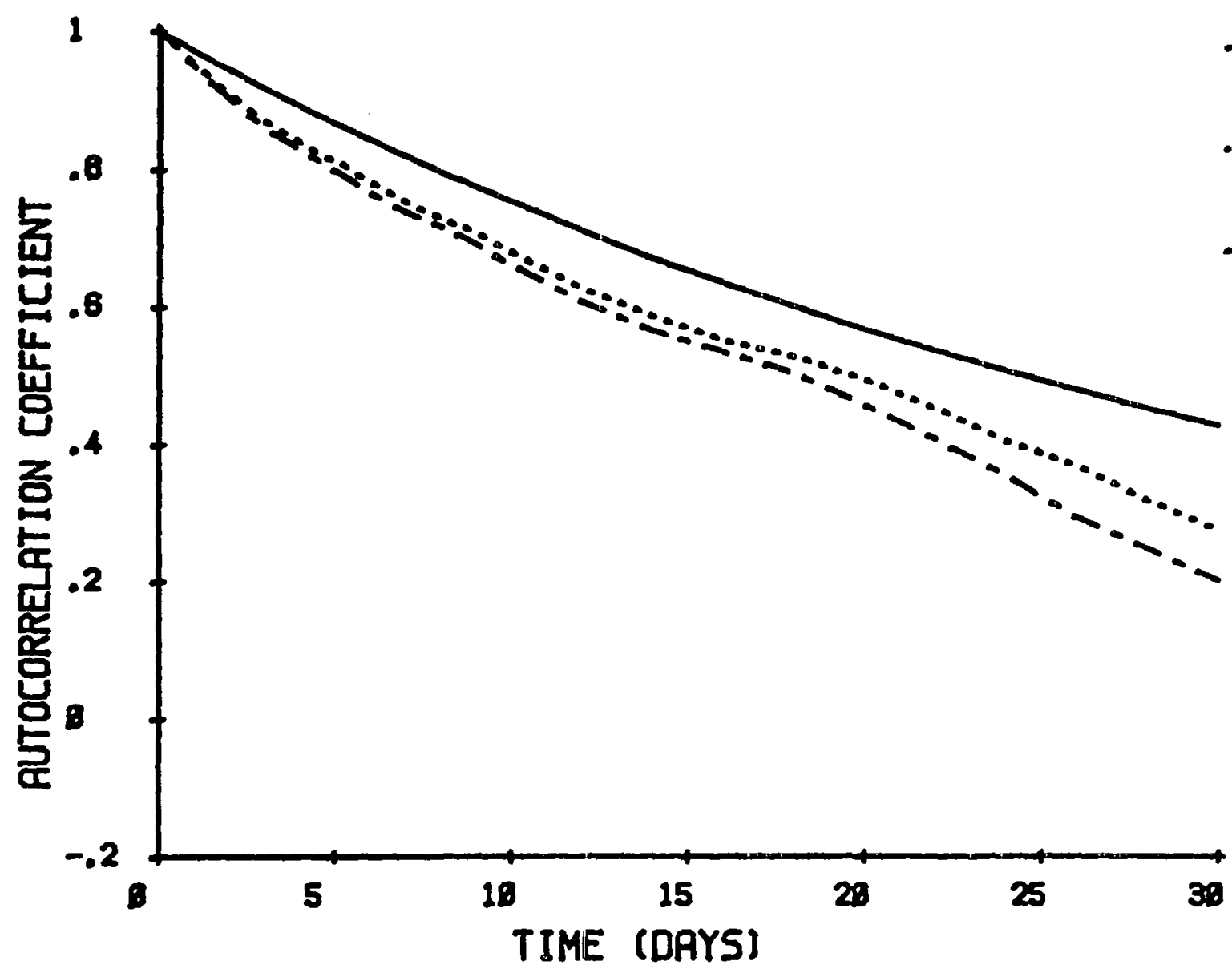

"COAFitete noDel

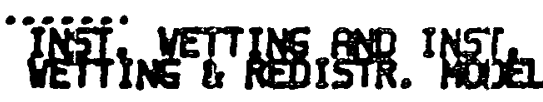

STOOASTIS MOOEL

Figure 31. Comparison of autocorrelation functions among the soil moisture dynamic and stochastic models for the Denver, Colorado climate

("deficit" case); clay loam soil. 
CHAPTER VIII

THE STOCHASTIC BEHAVIOR OF INITIAL SOIL MOISTURE

INTRODUCTION

The problem of establishing a stochastic representation of initial soil moisture can be approached from the general context of the evolutionary behavior of the entire soil moisture process. As indicated in the previous chapters, several fundamental phenomena produce the dynamic behavior of soil moisture. First, the random depth of infiltration during a storm event is responsible for "wetting" the surface layer of the soil. The randomness in the infiltration depth derives from random storm properties such as intensity and duration of which the depth is a function; infiltration is also a function of the hydraulic properties of the soil such as conductivity. Furthermore, the random rate of storm occurrence (or time between storms), the evapotranspiration (ET) rate and the properties of the soil itself are all important factors in producing variation in soil moisture behavior. The interstorm period and the ET rate are particularly important in the moisture depletion process and hence in establishing the initial soil moisture at the beginning of a subsequent storm event.

As a result of the random processes described above, the evolutionary behavior of soil moisture may be represented as shown schematically in Fig. 32. In this figure, $\theta_{0}$ is the moisture value at 


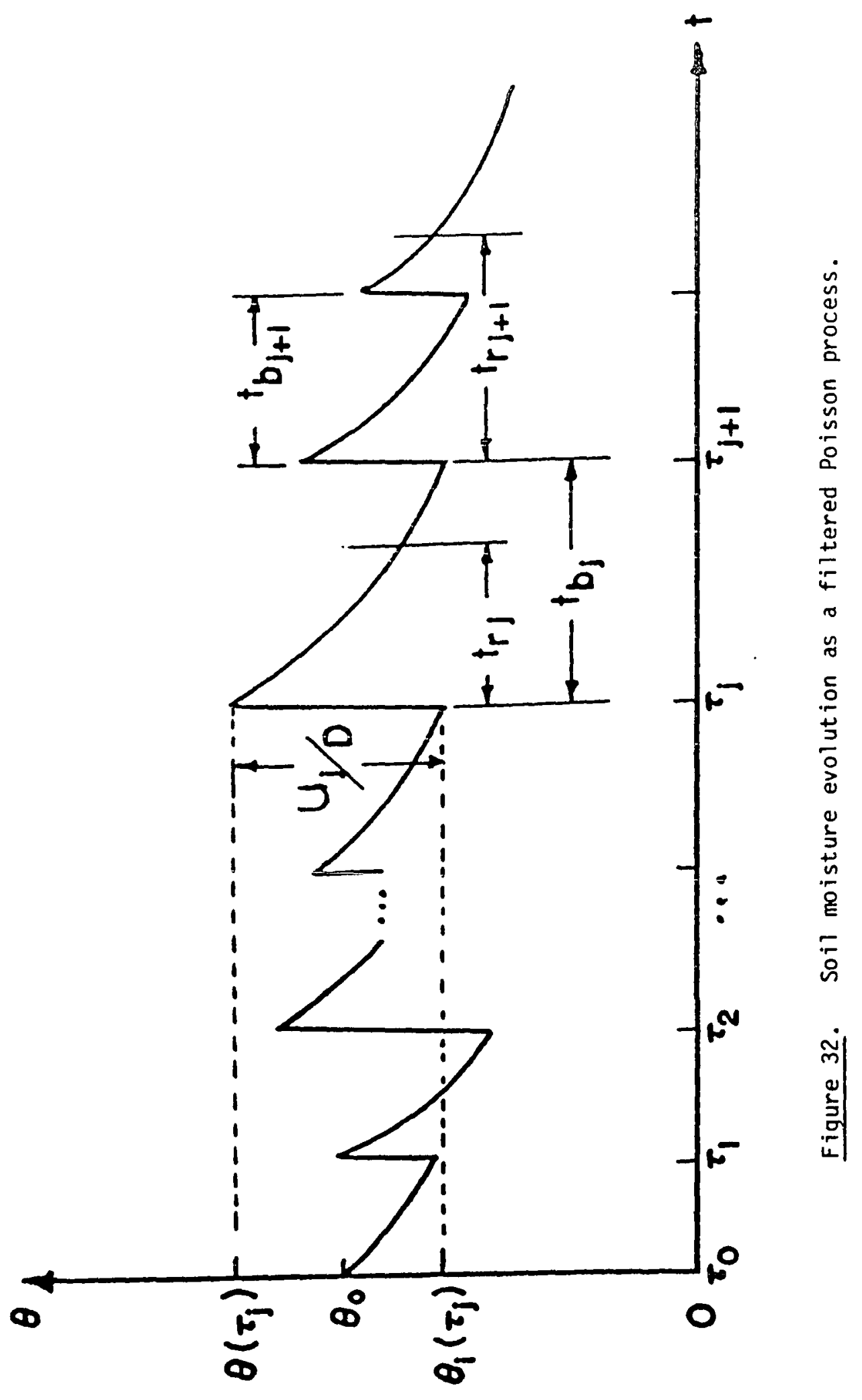


the beginning of an observation period; $\theta_{i}\left(\tau_{j}\right)$ is the $j$ th initial moisture value, that is, the soil water content just prior to the occurrence of the jth storm event; $U_{j}$ is the infiltration depth resulting from the $j$ th storm event, and $t_{b j}$ is the interstorm period. The sample path depicted in this figure has already been recognized to be that of a filtered Poisson process. A simple stochastic model of this form has been derived for the soil moisture process in Chapters $V$ and VI. The model also been presented by Koch and Mtundu (1986a). In the latter reference, two limiting modes of moisture behavior were also described. The first or "surplus", case was taken to represent the situation that would occur in a generally wet environment in which the storm events generate sufficient infiltration depth to induce drainage out of the root zone into the ground water system. In such a case, moisture depletion from the root zone during interstorm periods would result from both drainage and evapotranspiration. The second, or "deficit", case was taken to represent the opposite situation in which drainage out of the root zone does not occur due to the insufficient infiltration depth generated by the storm events. The moisture depletion process would, therefore, be driven by the evapotranspiration process only. Two analytical expressions describing these two limiting conditions were given as follows. For the "surplus" case:

$$
\begin{aligned}
\theta(t)=\theta_{r} & +\left[\theta_{j}\left(\tau_{j}\right)-\theta_{r}\right] e^{-\alpha\left(t-\tau_{j}\right)}-n\left[1-e^{-\alpha\left(t-\tau_{j}\right)}\right] \\
& +\frac{U_{j} e^{-\alpha\left(t-\tau_{j}\right)}}{D}, \quad \tau_{j} \leq t \leq \tau_{j+1}
\end{aligned}
$$


and for the "deficit" case:

$$
\begin{aligned}
\theta(t)=\theta_{w p} & +\left[\theta_{i}\left(\tau_{j}\right)-\theta_{w p}\right] e^{-\delta(t-\tau j)} \\
& +\frac{U_{j}}{D} e^{-\delta(t-\tau j)}, \quad \tau_{j} \leq t \leq \tau_{j+1}
\end{aligned}
$$

where all the variables are as defined previously.

Given Eq. 8.1 or 8.2 , the value of $\theta(t)$ at time $t=\tau_{j+1}$ represents the initial soil water content just prior to the next storm event. For instance, considering Eq. 8.1:

$$
\begin{aligned}
\theta\left(\tau_{j+1}\right)=\theta_{r} & {\left[\theta_{i}\left(\tau_{j}\right)-\theta_{r}\right] e^{\alpha\left(\tau_{j+1}-\tau_{j}\right)} } \\
& -n\left[1-e^{-\alpha\left(\tau_{j}+1-\tau_{j}\right)}\right]+\frac{U_{j} e^{-\alpha\left(\tau_{j+1}-\tau_{j}\right)}}{D}
\end{aligned}
$$

But $\tau_{j+1}-\tau_{j}=t_{b j}$, where $t_{b j}$ is the $j$ th interstorm period. Changing the notation somewhat to reflect the discrete nature of this equation and denoting the interstorm period simply by $T$, one obtains:

$$
\theta_{n+1}=\phi \theta_{n}+\varepsilon_{n+1}
$$

where $\theta_{n+1}$ and $\theta_{n}$ represent two consecutive initial moisture values and the other parameters are defined as follows:

$$
\phi=\phi(T)=e^{-\alpha T}
$$




$$
\varepsilon_{n+1}=\varepsilon_{n+1}(T)=\theta_{r}-n+\left(n-\theta_{r}+\frac{U_{n}}{D}\right) e^{-\alpha T}
$$

The variables $\theta_{n}, \theta_{n+1}, \quad U_{n}$ and $T$ are all random and, in particular, T has an exponential distribution. The distribution of the infiltration depth $U_{n}$ has been derived by a number of investigators, for example, Cordova and $\mathrm{Bras}(1981)$, who developed the distribution in the form of a Markov chain, and Koch (1982; see Eq. 5.20). The probability distribution of $\theta_{n}$, however, remains elusive at the present time and the literature does not reveal any attempts at its derivation. An empirical distribution is, subsequently, presented in this chapter.

In a similar manner, an equation of the same form can be obtained from Eq. 8.2 ("deficit" case). The parameters in this case are defined as follows:

$$
\phi=e^{-\delta T}
$$

and

$$
\varepsilon_{n+1}=\theta_{w p}+\left(\frac{U_{n}}{D}-\theta_{w p}\right) e^{-\delta T}
$$

Eq. 8.4 may be recognized to be a representation of the first-order autoregressive (AR) model. The only difference from the commonly used AR model is that, in this case, both the AR coefficient $\phi$ and the random component $\varepsilon$ are functions of the period ( $T$ ) separating the occurrence times of $\theta_{n}$ and $\theta_{n+1}$.

In the absence of a derived distribution, the autoregressive model given in Eq. 8.4 can be used to study the stochastic behavior of 
initial soil moisture. In particular, the moment properties of the initial moisture process are of interest and attention is next focused on these.

MOMENT PROPERTIES OF INITIAL SOIL MOISTURE

The first few moments, particularly the mean, variance and covariance, will be derived for the purpose of both qualifying and quantifying the dependence of initial soil moisture on parameters describing the physical and climatic systems. The "surplus" and "deficit" cases are considered separately because of the differences in the way they are modeled. In both cases, however, the evapotranspiration (ET), which is a random process, is considered to be fixed so the initial soil moisture must be thought of as being conditioned on ET. Furthermore, the moment derivations are based on the assumptions that the infiltration depth, $U$, has the distribution given by Eq. 5.20, whereas the interstorm period, $T$, is exponentially distributed with parameter $v$ (the reciprocal of $v$ being the mean interstorm period).

"Deficit" Case

Based on Eq. 8.4 with the parameters given by Eqs. 8.7 and 8.8 , the "deficit" case model can be re-written as:

$$
x_{n+1}=x_{n} e^{-\delta T}+\frac{U_{n}}{D} e^{-\delta T}
$$


where:

$$
x_{n+1}=\theta_{i}\left(\tau_{i}\right)-\theta_{w p}
$$

Based on the assumption that the initial soil moisture process is stationary in time, one can write:

$$
\operatorname{Er}\left[x_{n+1}\right]=\operatorname{Er}\left[x_{n}\right]=\operatorname{Er}[x], \quad r=1,2
$$

A similar assumption for the infiltration process gives:

$$
\operatorname{Er}\left[U_{n+1}\right]=\operatorname{Er}\left[U_{n}\right]=\operatorname{Er}[U], \quad r=1,2
$$

Using Eqs. 8.9 and 8.11 and noting that $x$ and $U$ are independent random variables and that both are independent of the exponentially distributed variable $T$, the mean, the variance and covariance can be established as follows:

$$
\begin{aligned}
& E[x]=\frac{\nu E[U]}{D \delta} \\
& \operatorname{Var}[x]=\frac{\nu E\left[U^{2}\right]}{2 D^{2} \delta} \\
& \operatorname{Cov}\left[x_{n}, x_{n+1}\right]=\frac{\nu}{\nu+\delta} \operatorname{Var}[x]
\end{aligned}
$$


The moments of $U$ are given by Eqs. 5.21 and 5.22 . From Eqs. 8.13 and 8.14, the correlation between successive values of $\theta_{i}$ is obtained as:

$$
\left.\operatorname{Corr} x_{n}, x_{n+1}\right]=\frac{v}{v+1}
$$

"Surplus" Case

From Eqs. 8.4, 8.5 and 8.6, one can write the "surplus" model as:

$$
x_{n+1}=x_{n} e^{-\alpha T}-n+n e^{-\alpha T}+\frac{U_{n}}{D} e^{-\alpha T}
$$

in which: $\quad x_{n+1}=\theta_{j}\left(\tau_{j}\right)-\theta_{r}$

Again, assuming stationarity in the processes and noting the independence between the variables $\chi, U$ and $T$, the moments in this case can be established as:

$$
\begin{aligned}
& E[X]=\frac{\nu}{D \alpha} E[U]-n \\
& \operatorname{Var}[x]=\frac{\nu}{2 D^{2} \alpha} E\left[U^{2}\right] \\
& \operatorname{Cov}\left[x_{n}, x_{n+1}\right]=\frac{v}{v+\alpha} \operatorname{Var}[x]+n^{2} \frac{\left[\nu^{2}(\nu-\alpha)\right]}{4 \alpha^{2}(\nu+\alpha)}
\end{aligned}
$$

where the first and second moments of $U$ are given by Eqs. 5.21 and 5.22. The correlation function of $x$ is obtained as the quotient of 
$\operatorname{Cov}[x]$ and $\operatorname{Var}[x]$, resulting in:

$$
\operatorname{Corr}\left[x_{n}, x_{n+1}\right]=\frac{v}{v+\alpha}+\frac{n^{2} v(v-\alpha)}{2 \alpha(v+\alpha) E\left[U^{2} / D^{2}\right]}
$$

In both the "deficit" and "surplus" cases, the mean and variance are seen to be the same as those of the stationary moments for the continuous soil moisture process, that is, Eqs. 8.12 and 8.13 are similar to Eqs. 7.5 and 7.7, respectively; whereas Eqs. 8.17 and 8.18 are similar to Eqs. 7.1 and 7.3, respectively. The covariances, and hence the correlations, are however different from those corresponding to the continuous soil moisture process; of course, this is a reflection of the discrete nature of the initial soil moisture process. It is apparent, therefore, that the average properties of the initial soil moisture are not distinct from those of the primary process. A separate evaluation of the the initial moisture process is, thus, not called for except to proceed to investigate its possible probability distribution function. The purpose of such an investigation has been alluded to in Chapter II in connection with accounting for sensitivity of most hydrologic processes (e.g. infiltration, precipitation excess and hence streamflow) to initial soil moisture conditions in the watershed (Eq. 2.1). Furthermore, the primary soil moisture process itself has been shown in Chapter VII to be somewhat sensitive to the initial water content of the soil. 


\section{DISTRIBUTION OF INITIAL SOIL MOISTURE}

Ultimately, the probability distribution function of initial soil moisture is of interest, as observed in the introductory section. Currently, however, an analytical derivation of the distribution does not appear possible due to the complexity of the moisture model itself and that of infiltration.

Inasmuch as a distribution derived on the basis of physical principles is precluded by the complexity of the initial moisture model, a practical, alternative procedure is to simply fit a known distribution empirically. In this particular case, the form of the distribution was first suggested by virtue of the fact that soil moisture has both an upper and lower bound. The beta distribution has such properties and, hence, was the one selected. This selection was subsequently verified by comparing histograms of initial moisture values (generated by Monte Carlo simulation procedures) with the calibrated empirical distribution. Since two limiting modes of initial moisture behavior have been identified, each limiting mode is associated with its own beta distribution. In other words, the parameters of the distribution corresponding to the "surplus" case are numerically different from those corresponding to the "deficit" case.

The Beta Distribution

The beta distribution is generally defined over the interval 0 to 1 (e.g. Hahn and Shapiro, 1967). In view of this, the probability density functions (PDF's) for the "surplus" (or "excess") and "deficit" 
cases would, respectively, be given by:

$$
\begin{aligned}
& f_{\theta_{e}^{*}}\left(\theta^{*}\right)=\frac{\Gamma\left(\alpha_{e}+\beta_{e}\right)}{\Gamma\left(\alpha_{e}\right) \Gamma\left(\beta_{e}\right)}\left(\theta_{e}^{*}\right)^{\alpha_{e}-1}\left(1-\theta_{e}^{*}\right)^{\beta_{e}-1}, 0 \leq \theta_{e}^{*} \leq 1 \\
& f_{\theta_{d}^{*}}\left(\theta^{*}\right)=\frac{\Gamma\left(\alpha_{d}+\beta_{d}\right)}{\Gamma\left(\alpha_{d}\right) \Gamma\left(\beta_{d}\right)}\left(\theta_{d}^{*}\right)^{\alpha_{d}-1}\left(1-\theta_{d}^{*}\right)^{\beta_{d}-1,}, 0 \leq \theta_{d}^{*} \leq 1
\end{aligned}
$$

in which $\Gamma(\cdot)$ denotes the gamma function and where $\alpha$ and $\beta$ are parameters of the beta distribution which take only positive values, and the subscripts $e$ and d denote the "excess" and "deficit" situations, respectively. The initial moisture in Eqs. 8.21 and 8.22 is given in a normalized form; that is:

$$
\theta^{*}=\frac{{ }^{\theta_{i}}-\theta_{r}}{\theta_{s}-\theta_{r}}
$$

and

$$
\theta_{d}=\frac{\theta_{j}-\theta_{w p}}{\theta_{r}-\theta_{w p}}
$$

The beta PDF's may, of course, be generalized from those restricted to the unit interval $(0,1)$ to those covering any interval $(a, b)$, where $a$ and $\mathrm{b}$ are the appropriate lower and upper bounds, respectively, by using the monotonic transformation (Hahn and Shapiro, 1967): 


$$
\theta_{i}=(b-a) \theta^{*}+a
$$

where $\theta^{*}$ is beta-distributed according to Eq. 8.21 or 8.22 . The resulting pdf's are then given by:

$$
\begin{gathered}
g_{\theta_{i}}\left(\theta_{i}\right)=\frac{1}{\left(\theta_{s}-\theta_{r}\right)^{\alpha} e^{+\beta_{e}-1}} \frac{\Gamma\left(\alpha_{e}+\beta_{e}\right)}{\Gamma\left(\alpha_{e}\right) \Gamma\left(\beta_{e}\right)}\left(\theta_{i}{ }^{-\theta_{r}}\right)^{\alpha_{e}-1}\left(\theta_{s}-\theta_{i}\right)^{\beta_{e}-1,}, \\
\text { for } \quad \theta_{r} \leq \theta_{i} \leq \theta_{s}
\end{gathered}
$$

for the "surpius" case, and:

$$
\begin{gathered}
g_{\theta_{i}}(\theta)=\frac{1}{\left(\theta_{r}-\theta_{w p}\right)^{\alpha_{d}+\beta_{d}-1}} \frac{\Gamma\left(\alpha_{d}+\beta_{d}\right)}{\Gamma\left(\alpha_{d}\right) \Gamma\left(\beta_{d}\right)}\left(\theta_{i}{ }^{-\theta_{w p}}\right)^{\alpha_{d}-1}\left(\theta_{r}-\theta_{i}\right)^{\beta_{d}-1}, \\
\text { for } \quad \theta_{w p} \leq \theta_{i} \leq \theta_{r}
\end{gathered}
$$

for the "deficit" case. Hahn and Shapiro (1967), among others, give the basic form of the beta pdf.

Given that the parameters $\alpha$ and $\beta$ are known, the mean and variance of the initial moisture would then be given, respectively, by:

$$
\begin{aligned}
& E\left[\theta^{*}\right]=\frac{\alpha}{\alpha+\beta} \\
& \operatorname{Var}\left[\theta^{\star}\right]=\frac{\alpha \beta}{(\alpha+\beta+1)(\alpha+\beta)^{2}}
\end{aligned}
$$


Estimation of the Beta Distribution Parameters

The mean and variance equations ( 8.28 and 8.29 ) may be used to obtain the moment estimators for the parameters $\alpha$ and $\beta$ provided initial moisture data (observed or simulated) are available. This approach is subsequently illustrated in this chapter where the data were generated via Monte Carlo simulations. Alternatively, $\alpha$ and $\beta$ can be estimated a priori from the hydraulic properties of the soil and the characteristics of the climate. Such physically based parameters may be derived from the stochastic model of initial soil moisture presented earlier.

Using the latter approach, Eqs. 8.12 and 8.13 for the "deficit" case and Eqs. 8.17 and 8.18 for the "surplus" case are used directly to obtain the mean and variance as follows. For the "deficit" case, the mean and variance are:

$$
\begin{aligned}
E\left[\theta^{*}\right] & =\frac{\nu E[U]}{D \delta\left(\theta_{r}-\theta_{W p}\right)} \\
\operatorname{Var}\left[\theta^{*}\right] & =\frac{\nu E\left[U^{2}\right]}{20^{2} \delta\left(\theta_{r}-\theta_{w p}\right)^{2}}
\end{aligned}
$$

For the "surplus" case, the corresponding equations are:

$$
\begin{aligned}
E\left[\theta^{*}\right] & =\frac{\nu E[U]}{D a\left(\theta_{s}-\theta_{r}\right)} \\
\operatorname{Var}\left[\theta^{*}\right] & =\frac{\nu E\left[U^{2}\right]}{2 D^{2} \alpha\left(\theta_{s}-\theta_{r}\right)^{2}}
\end{aligned}
$$


Upon substitution of Eqs. 8.30 and 8.31 into Eqs. 8.28 and 8.29 and solving the resulting simultaneous equations, one obtains:

$$
\begin{aligned}
& \beta_{d}=\frac{(1-\mu)\left[\mu(1-\mu)-\sigma^{2}\right]}{\sigma^{2}} \\
& \alpha_{d}=\frac{B \mu}{1-\mu}
\end{aligned}
$$

where $\mu$ and $\sigma^{2}$ are, respectively, the mean and variance of the standardized initial moisture as given by Eqs. 8.30 and 8.31 . The parameters $\alpha_{e}$ and $\beta_{e}$ for the "surplus" case are obtained in a similar fashion and their formulas have the same form as Eqs. 8.34 and 8.35 except that $\mu$ and $\sigma^{2}$ are as given by Eqs. 8.32 and 8.33.

Verification of the Beta Distribution Via Monte Carlo Simulations and Goodness-of-Fit Tests

The same climatic environments were selected for the purpose of carrying out Monte Carlo simulations to generate sequences of initial moisture that were to be subjected to statistical analyses. The dynamic soil moisture model presented by Koch et al (1986) and outlined in Chapter $V$ was used to generate the random initial moisture values.

Different soil conditions were represented by selecting three soil types, namely, clay loam, loam and sandy $10 \mathrm{am}$, to be tested under the same two different climatic regimes considered before. The parameters of the climate and the soil properties used in this respect are, again, those given in Tables I and II (Chapter VI), respectively.

The model verification was performed on the basis of normalized 
initial moisture values (Eq̣s. 8.23 and 8.24) and both parametric and non-parametric tests were undertaken. In either case, the hypothesis that the beta distribution adequately described the sets of simulated observations was tested against the alternative hypothesis that the pdf of initial moisture was not of the beta-type. The parameters of the beta distribution, $\alpha$ and $\beta$, were estimated from the generated initial moisture data using the method of moments. In this case, equations of the form (8.34) and (8.35) are used except that $\mu$ and $\sigma^{2}$ are replaced by the corresponding sample mean and variance, respectively.

As mentioned previously, the beta distribution as a model that would adequately describe the behavior of initial moisture was also suggested by the near agreement between the cumulative relative frequency curve and the theoretical cumulative distribution function obtained using the estimated parameters. The results of such visual-judgement tests are given in Figs. 33 to 41 for the various cases, including different climatic environments ( $B$ lacksburg versus Denver), different soil types (clay loam, $10 \mathrm{am}$ and sandy 10am) and the "surplus" and "deficit" cases.

The non-parametric Kolmogorov-Smirnov (K-S) test was next performed on the generated data to verify the beta distribution. The results of this test are summarized in Tables IV to VI, which also give typical values of the moment estimators of the parameters $a$ and $\beta$. The $K-S$ statistic is denoted by $d_{1}$ whereas the critical values are given as $c_{n, \alpha}$ where $n$ is the sample size and $a$ is the significance level. Comparison of the K-S statistic and critical values at several values of $\alpha(0.10,0.05,0.01)$ shows that the hypothesis of a beta distribution 


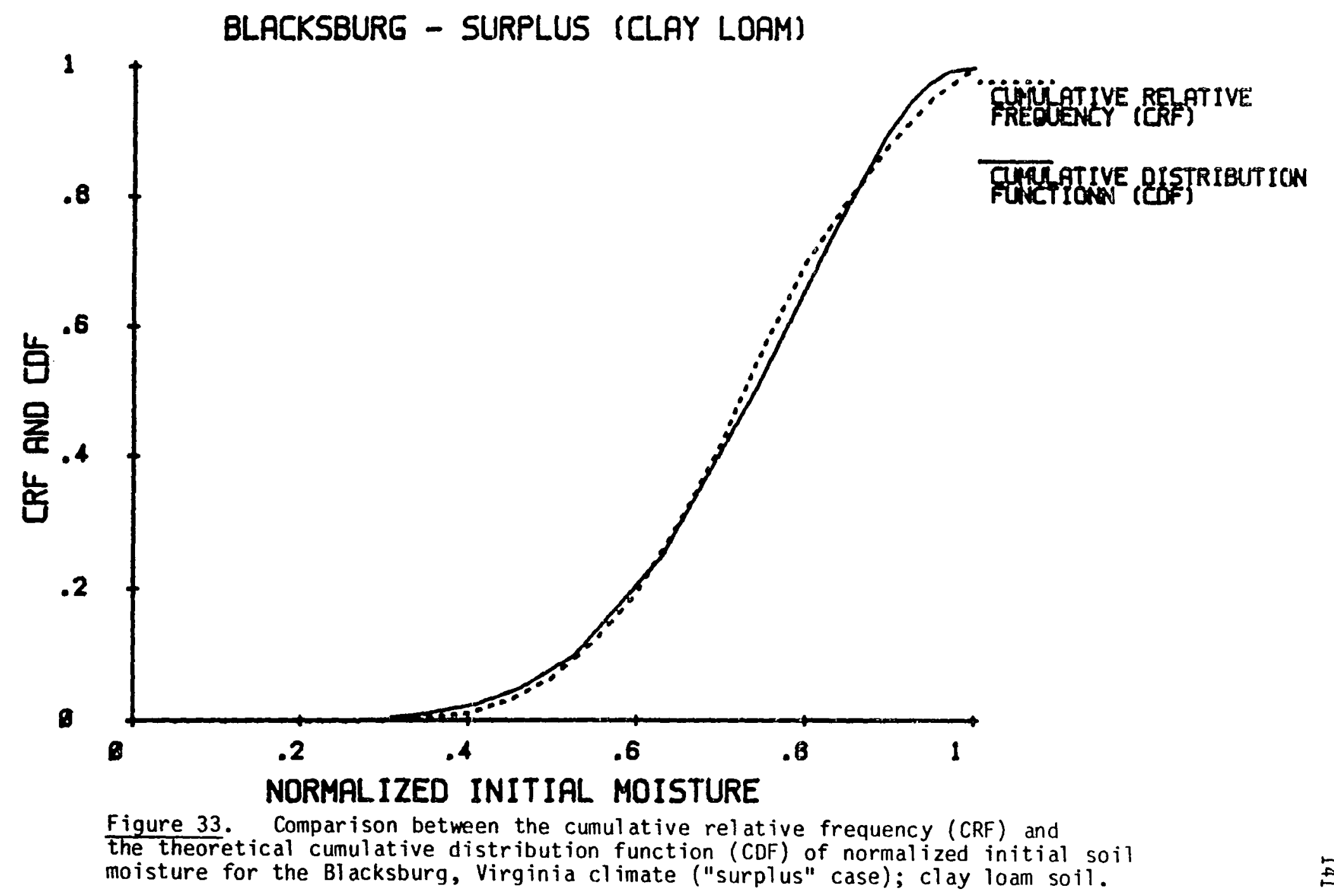




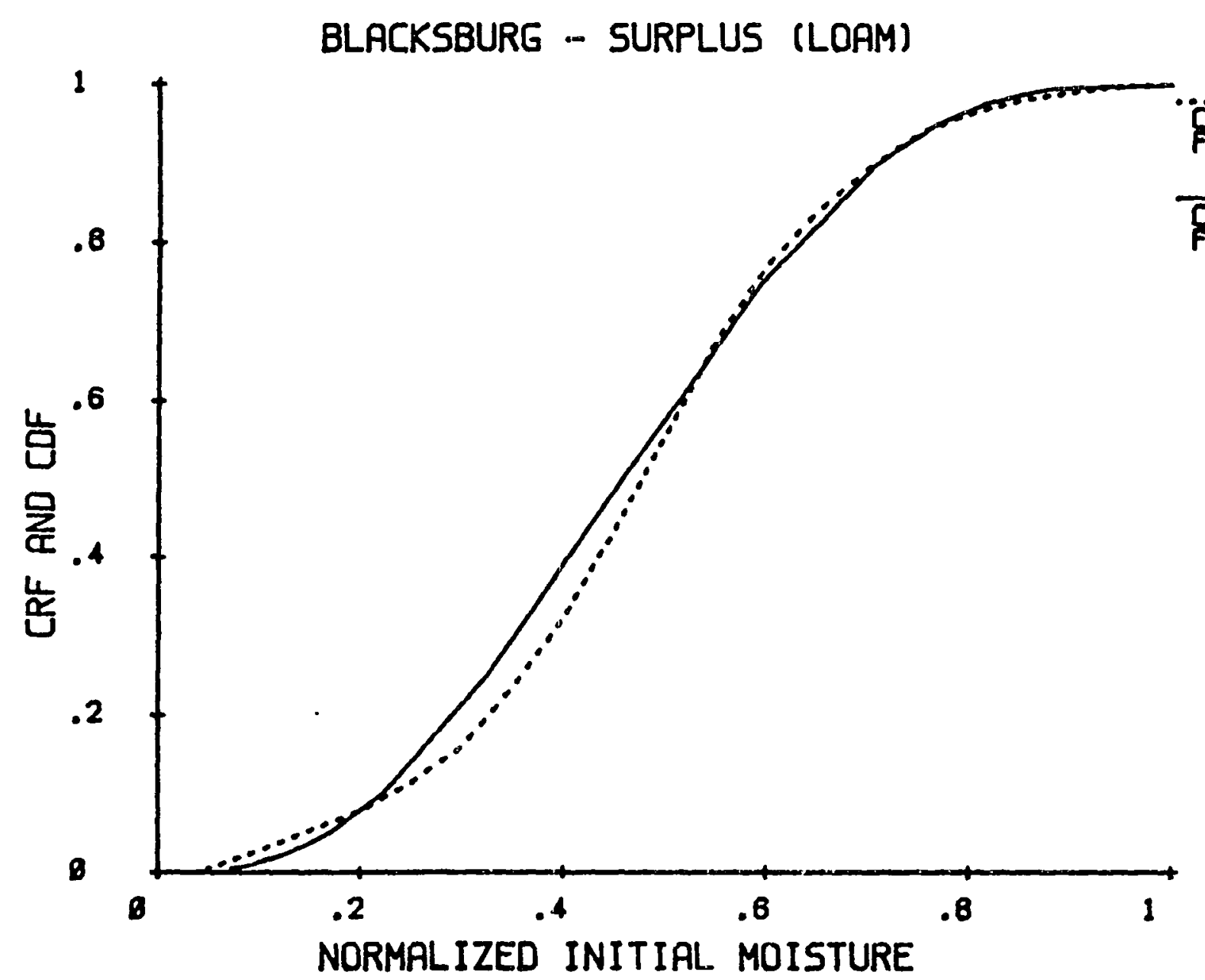

Figure 34. Comparison between the cumulative relative frequency (CRF) and the theoretical cumulative distribution function (CDF) of normalized initial soil moisture for the Blacksburg, Virginia climate ("surplus" case); loam soil. 


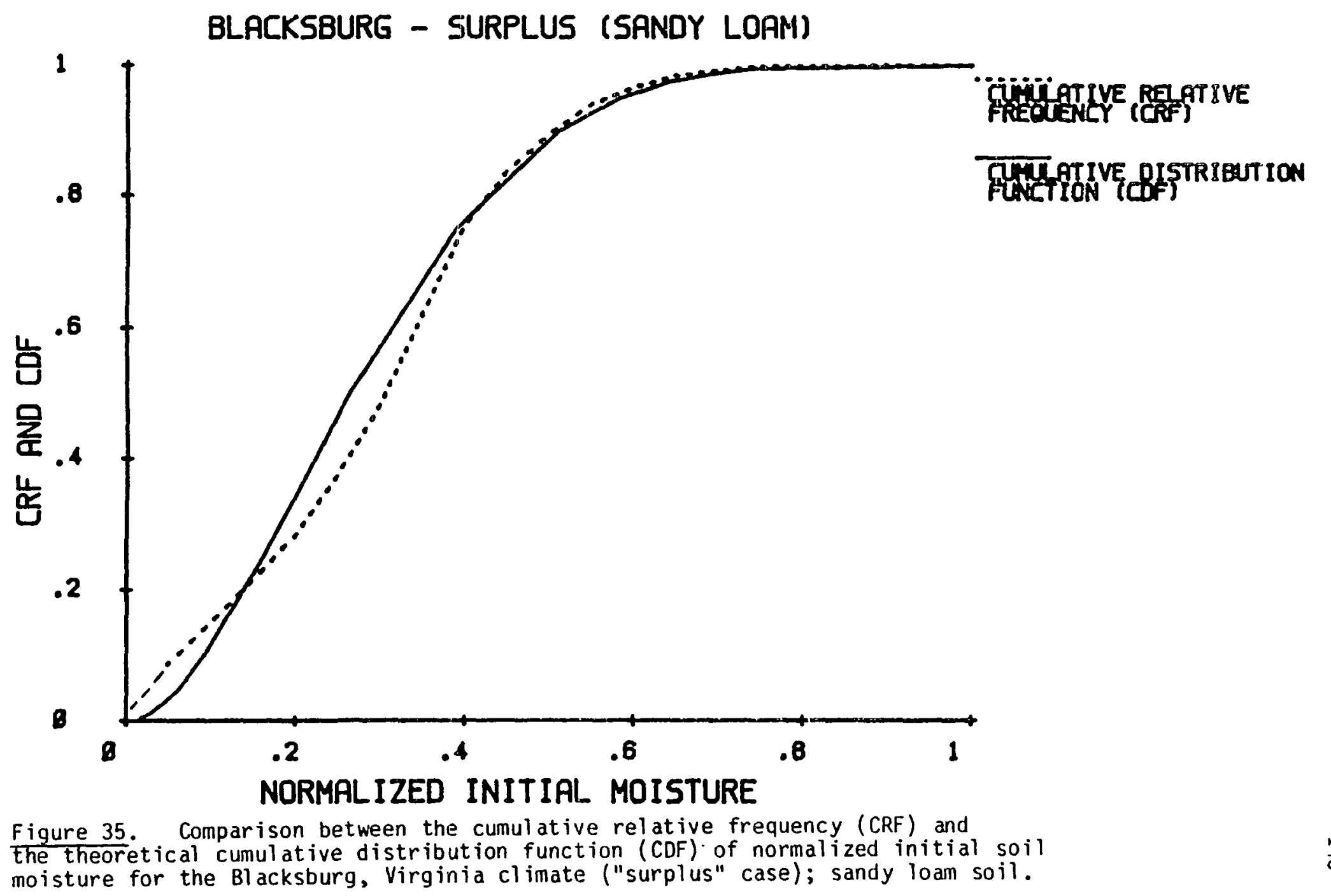




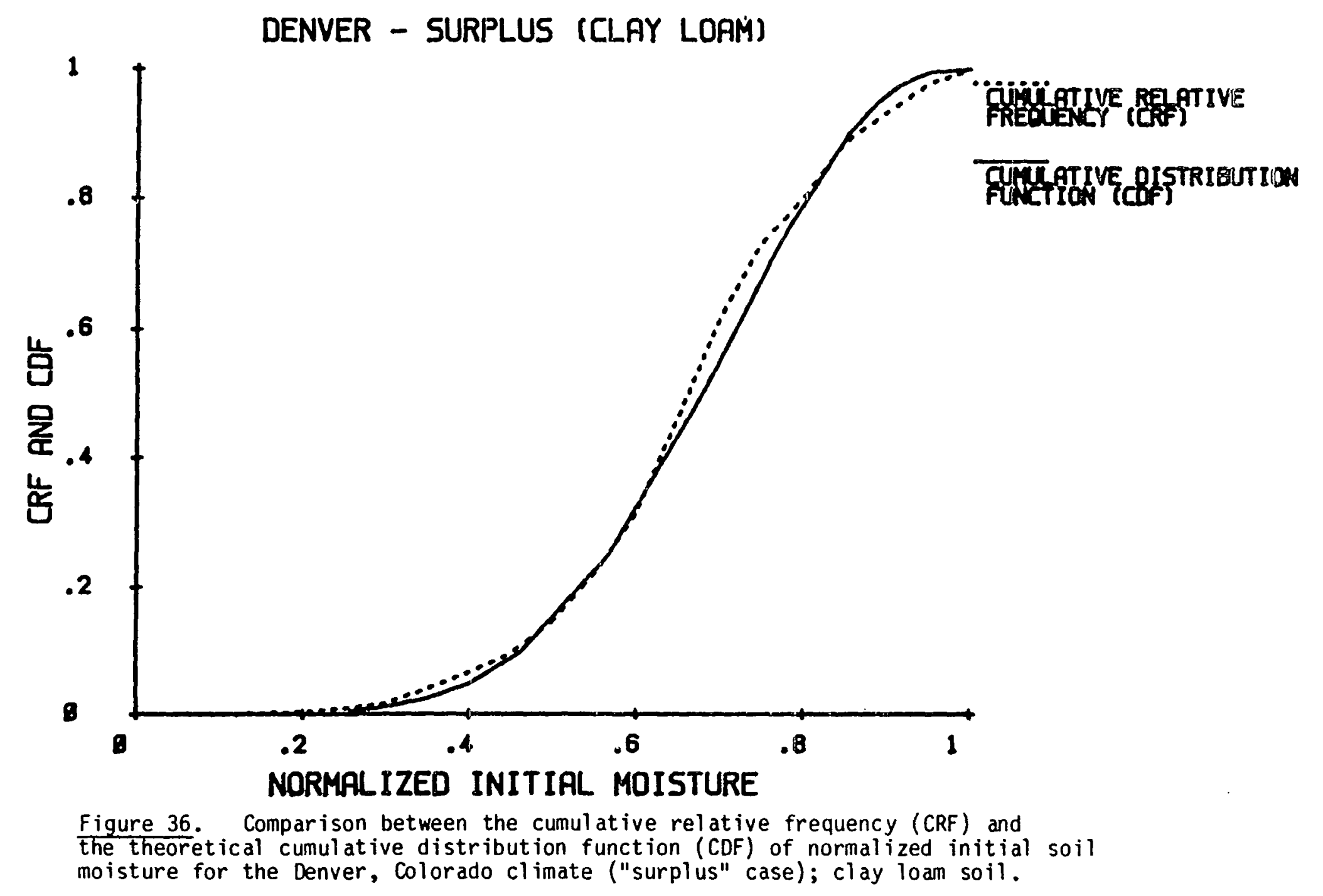




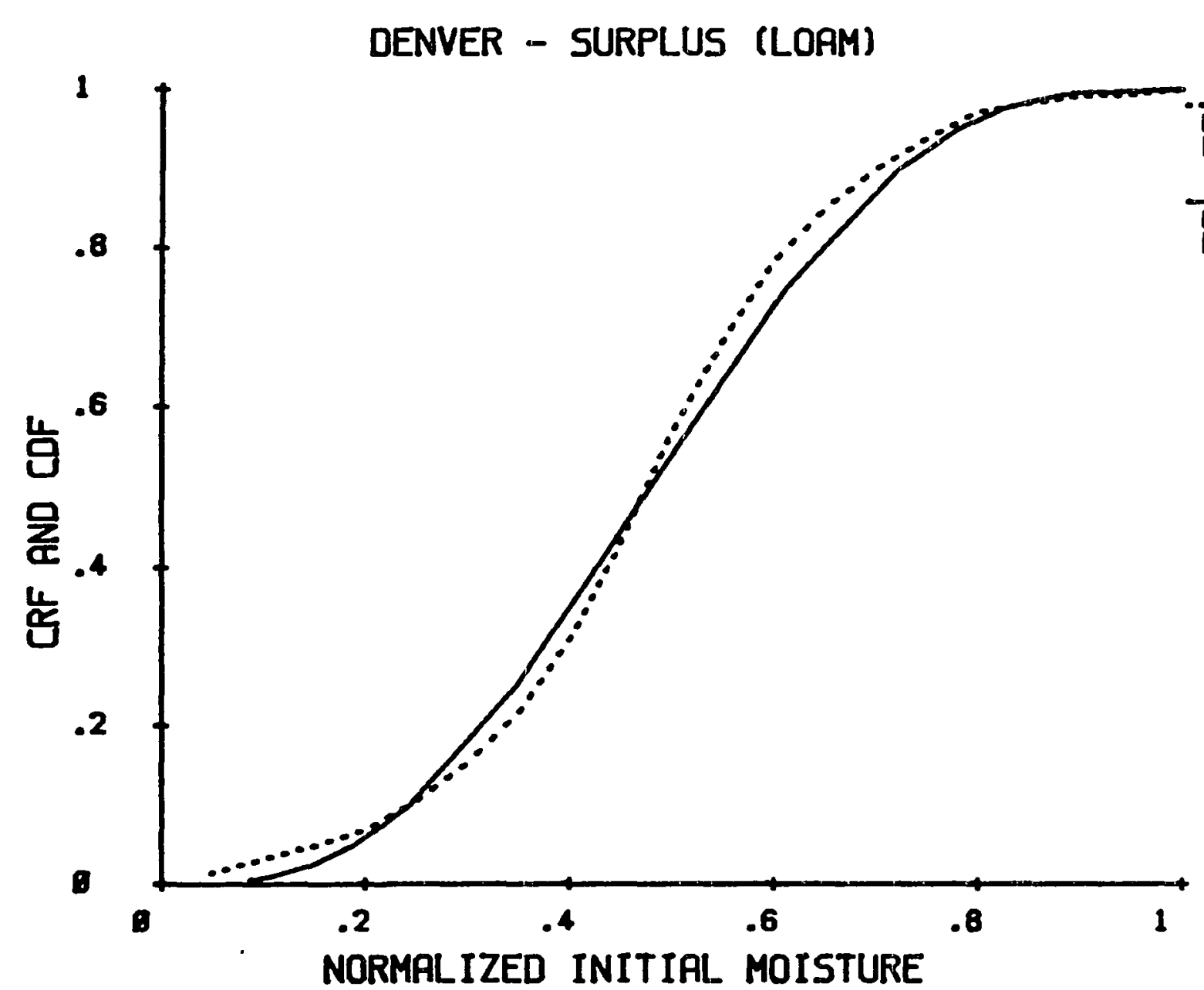

Figure 37. Comparison between the cumulative relative frequency (CRF) and the theoretical cumulative distribution function (CDF) of normalized initial soil moisture for the Denver, Colorado climate ("surplus" case); loam soil.

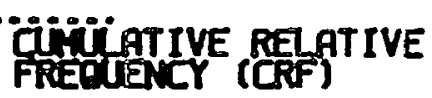

GTH RTIVE DISTRIBUTION 


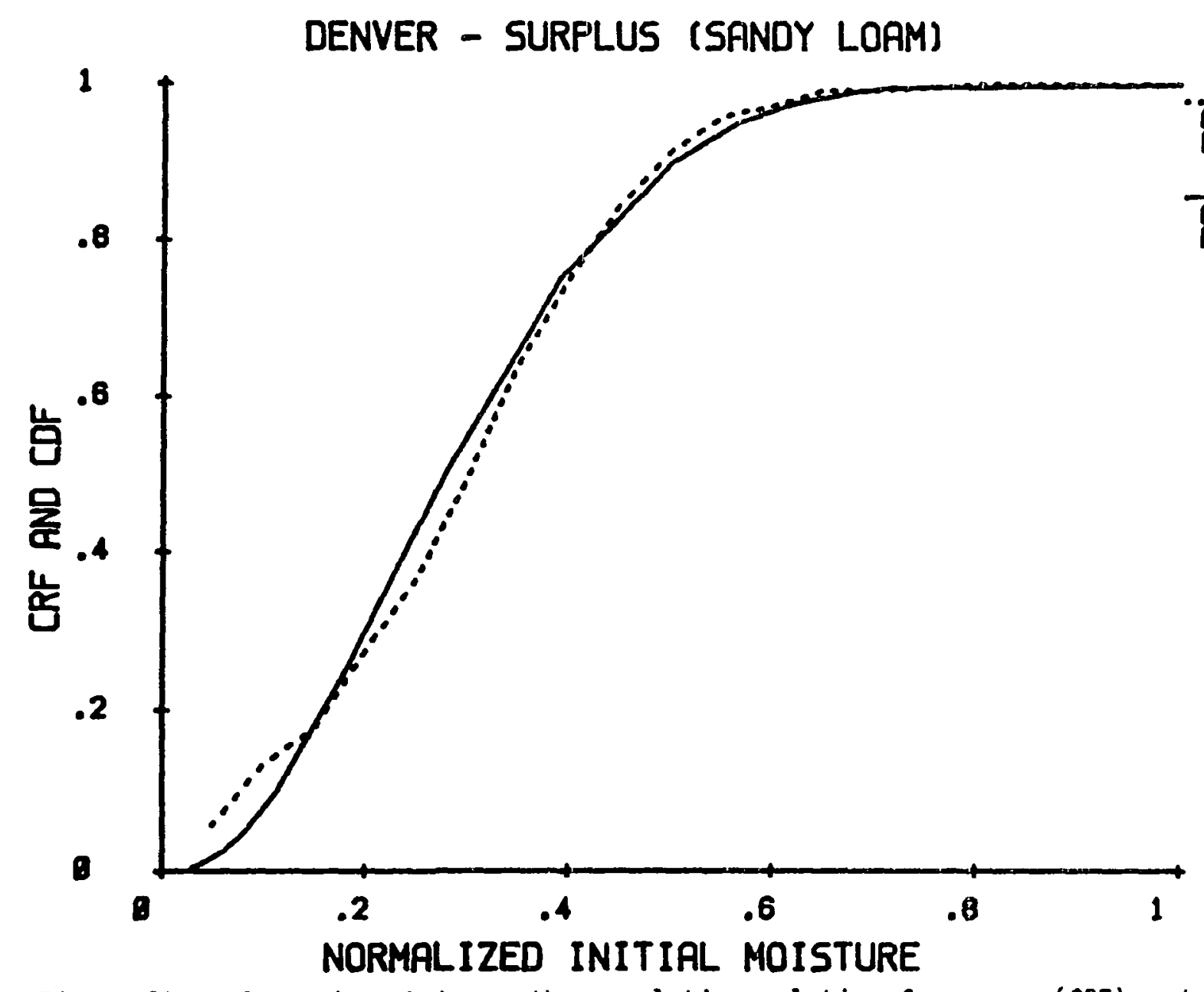

Figure 38. Comparison between the cumulative relative frequency (CRF) and the theoretical cumulative distribution function (CDF) of normalized initial soil moisture for the Denver, Colorado climate ("surplus" case); sandy loam soil. 


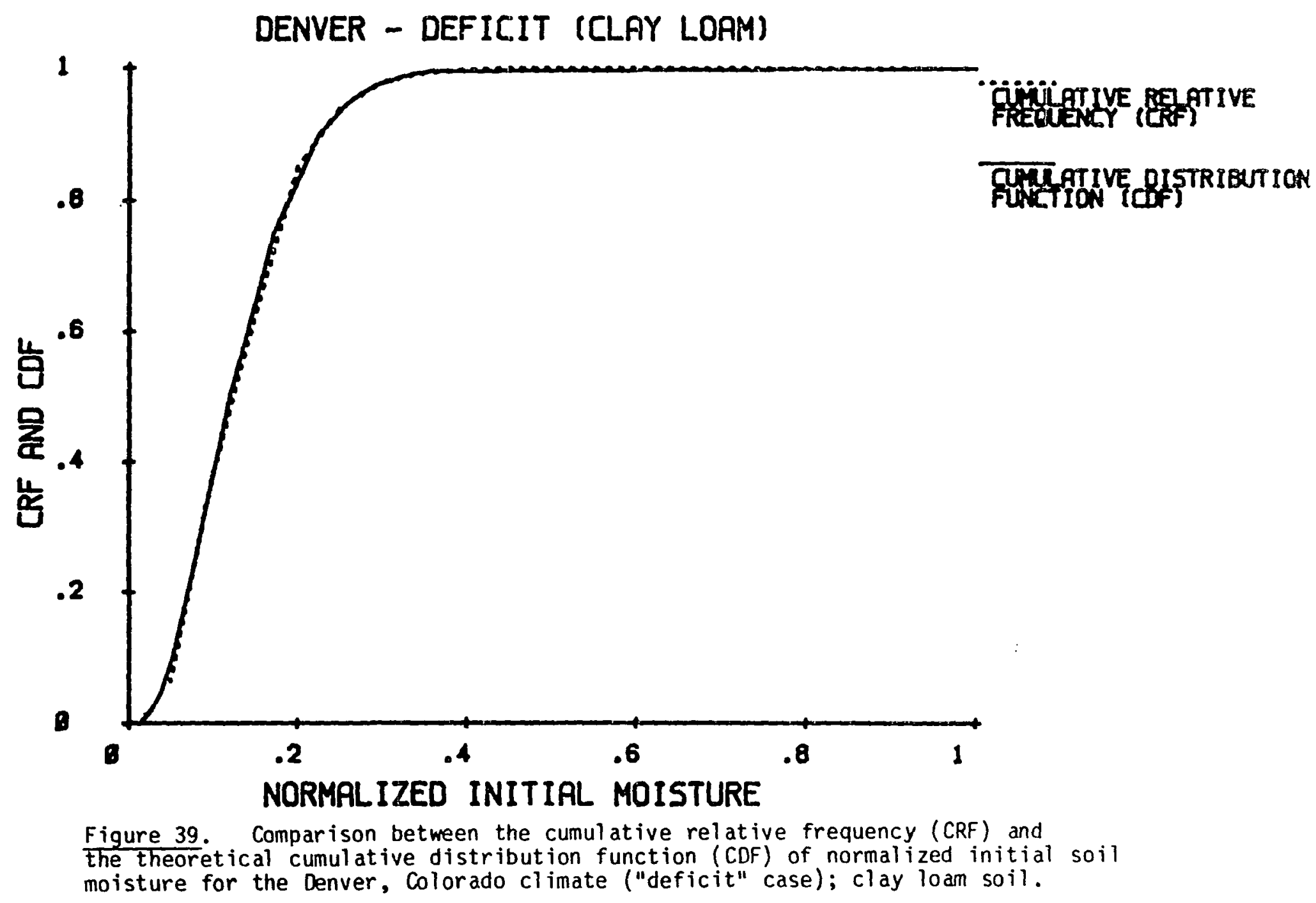




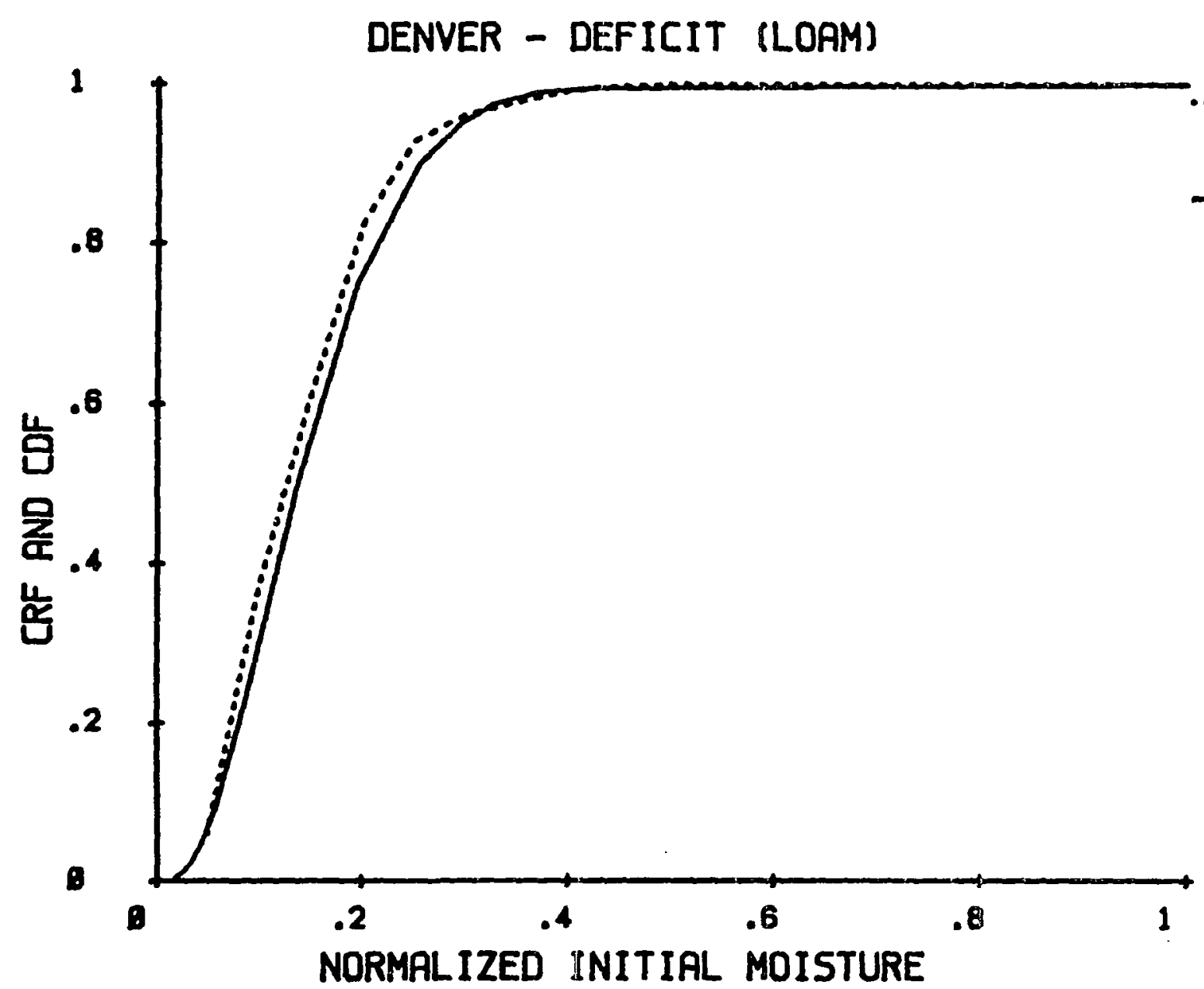

Quịi:nTIVE RELTIVE

\section{FUNCATIVE RISTRIBUTION}

the theoretical cumulative distribution function (CDF) of normalized initial soil

moisture for the Denver, Colorado climate ("deficit" case); loam soil. 


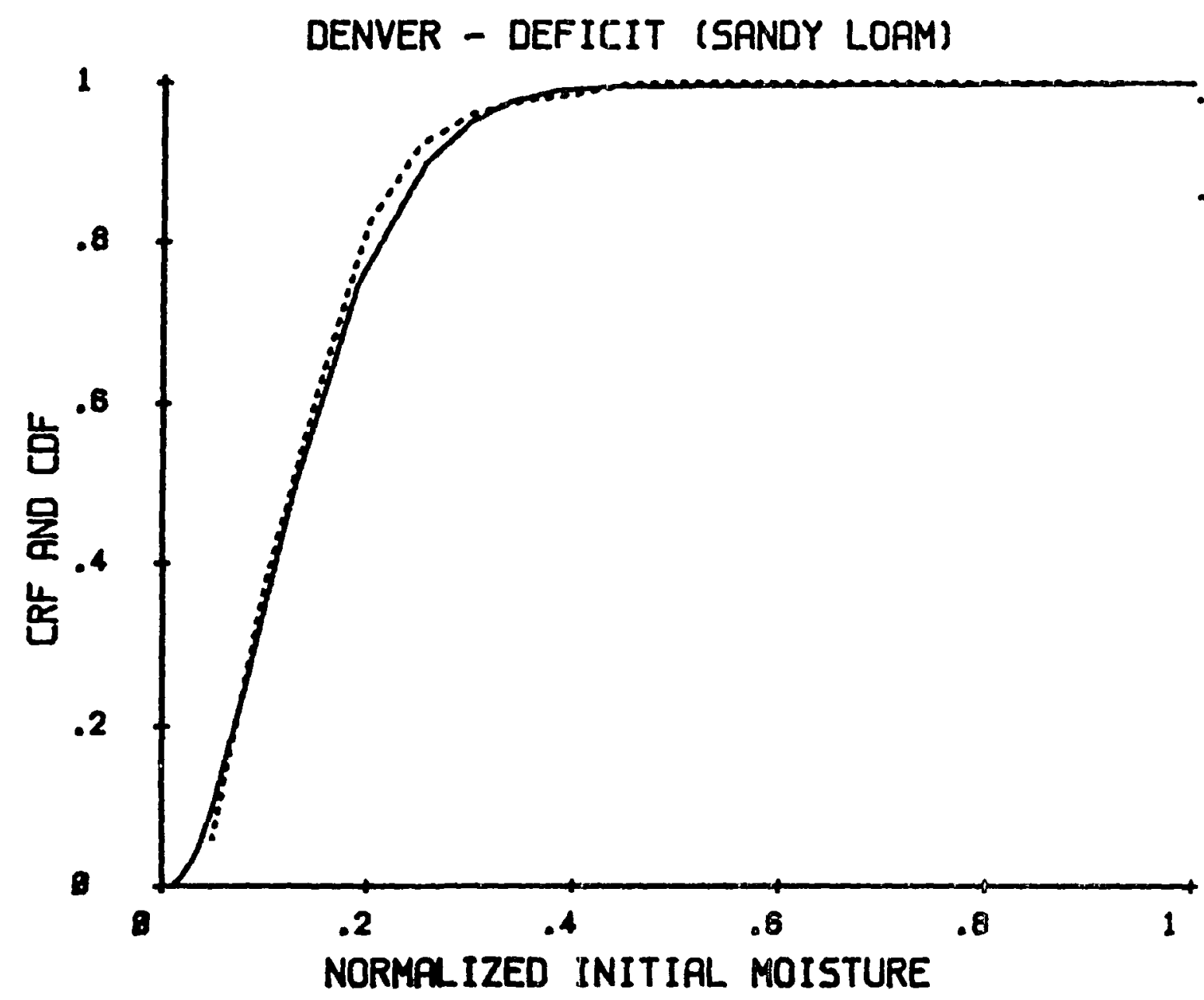

Figure 41. Comparison between the cumul ative relative frequency (CRF) and the theoretical cumulative distribution function (CDF) of normalized initial soil moisture for the Denver, Colorado climate ("deficit" case); sandy loam soil. civiüntrive Ren ative FUTARIVE DISTRIBUTION 
TABLE IV

KOLMOGOROV-SMIRNOV TEST RESULTS FOR THE BLACKSBURG, VA. CLIMATE: "SURPLUS" CASE

\begin{tabular}{|c|c|c|c|c|c|c|c|}
\hline \multirow{2}{*}{ Type of soil } & \multicolumn{2}{|c|}{$\begin{array}{l}\text { Beta-pdf } \\
\text { Parameters }\end{array}$} & \multirow{2}{*}{$\begin{array}{c}\text { Sample } \\
\text { size } \\
n\end{array}$} & \multirow{2}{*}{$\begin{array}{c}\mathrm{k}-\mathrm{S} \\
\text { Statistic } \\
\mathrm{d}_{1}\end{array}$} & \multicolumn{3}{|c|}{ Critical Values } \\
\hline & $a_{e}$ & $\mathrm{Be}$ & & & $c_{n}, 0.10$ & $c_{n}, 0.05$ & $c_{n}, 0.01$ \\
\hline $\mathrm{Cl}$ ay loam & 5.93 & 2.24 & 76 & 0.0746 & 0.140 & 0.156 & 0.187 \\
\hline Loam & 2.56 & 2.75 & 76 & 0.0746 & 0.140 & 0.156 & 0.187 \\
\hline Sandy loam & 1.84 & 4.13 & 74 & 0.0894 & 0.142 & 0.158 & 0.189 \\
\hline
\end{tabular}


TABLE V

KOLMOGOROV-SMIRNOV TEST RESULTS FOR THE DENVER, CO. CLIMATE: "SURPLUS" CASE

\begin{tabular}{|c|c|c|c|c|c|c|c|}
\hline \multirow{2}{*}{ Type of soil } & \multicolumn{2}{|c|}{$\begin{array}{l}\text { Beta-pdf } \\
\text { Parameters }\end{array}$} & \multirow{2}{*}{$\begin{array}{c}\text { Sample } \\
\text { size } \\
n\end{array}$} & \multirow{2}{*}{$\begin{array}{c}K-S \\
\text { Statistic } \\
d_{1}\end{array}$} & \multicolumn{3}{|c|}{ Critical Values } \\
\hline & $\alpha_{e}$ & $\mathrm{Be}_{\mathrm{e}}$ & & & $c_{n}, 0.10$ & $c_{n}, 0.05$ & $c_{n}, 0.01$ \\
\hline Clay loam & 5.24 & 2.72 & 76 & 0.0457 & 0.140 & 0.156 & 0.187 \\
\hline Loam & 3.06 & 3.46 & 75 & 0.0767 & 0.141 & 0.157 & 0.188 \\
\hline Sandy loam & 2.20 & 5.19 & 64 & 0.0716 & 0.153 & 0.170 & 0.204 \\
\hline
\end{tabular}


TABLE VI

KOLMOGOROV-SMIRNOV TEST RESULTS FOR THE DENVER, CO. CLIMATE:

"DEF ICIT" CASE

\begin{tabular}{|c|c|c|c|c|c|c|c|}
\hline \multirow{2}{*}{ Type of Soil } & \multicolumn{2}{|c|}{$\begin{array}{l}\text { Beta-pdf } \\
\text { Par ameters }\end{array}$} & \multirow{2}{*}{$\begin{array}{c}\text { Sample } \\
\text { size } \\
n\end{array}$} & \multirow{2}{*}{$\begin{array}{c}K-S \\
\text { Statistic } \\
d_{1}\end{array}$} & \multicolumn{3}{|c|}{ Critical Values } \\
\hline & $\alpha_{e}$ & $\mathrm{Be}_{\mathrm{e}}$ & & & $c_{n}, 0.10$ & $c_{n}, 0.05$ & $c_{n}, 0.01$ \\
\hline Clay loam & 3.72 & 22.82 & 277 & 0.0585 & 0.0733 & 0.0817 & 0.0979 \\
\hline Loam & 3.00 & 17.20 & 277 & 0.0526 & 0.0733 & 0.0817 & 0.0979 \\
\hline Sandy loam & 2.73 & 15.41 & 277 & 0.0621 & 0.0733 & 0.0817 & 0.0979 \\
\hline
\end{tabular}


would be accepted at the $10 \%$ significance level even after taking into account the fact that the parameters were estimated from the data.

In view of the conservative nature of the K-S test when the pdf parameters are estimated, the parametric chi-square $\left(x^{2}\right)$ test was also performed on the generated data in an attempt to increase confidence in the hypothesized distribution. The test results are summarized in Table VII. As can be seen from this table, the hypothesis of a beta distribution would be accepted at the $5 \%$ significance level.

Comparison of Physically Based Beta Distribution Parameters with Moment Estimators

As previously observed, the parameters $\alpha$ and $\beta$ of the beta distribution could be estimated a priori from the properties of the climate and soils as indicated in Eqs. 8.34 and 8.35. Using these equations, the physically based parameters were computed on the basis of mean and variance values calculated from Eqs. 8.30 and 8.31 for the "deficit" case and Eqs. 8.32 and 8.33 for the "surplus" case in order to compare them with those based on simulated mean and variance values. The comparisons are presented in Table VIII for the Blacksburg, Va. cl imate ("surplus" case) and the Denver, Co. climate ("deficit" case).

The results in Table VIII indicate that the physically based $a$-values are in fairly close agreement with the simulated ones for the deficit case whereas, for the "surplus" case, it is the $\beta$-values which are in reasonable agreement. Lack of agreement betwen simulated and physically based values can be explained by a number of factors. The first of these is the fact that the stochastic model used to predict the 
TABLE VII

CHI-SQUARE TEST RESULTS FOR INITIAL SOIL MOISTURE

\begin{tabular}{|c|c|c|c|}
\hline Location & Type of Soil & $\underset{n}{\text { Sample size }}$ & $\begin{array}{l}\text { Chi-square } \\
\text { Statistic }\end{array}$ \\
\hline $\begin{array}{l}\text { Bl acksburg, Va. } \\
\text { "Surplus" case }\end{array}$ & $\begin{array}{l}\text { Clay Loam } \\
\text { Loam } \\
\text { Sandy Loam }\end{array}$ & $\begin{array}{l}76 \\
76 \\
74\end{array}$ & $\begin{array}{r}10.8 \\
5.31 \\
7.08\end{array}$ \\
\hline $\begin{array}{l}\text { Denver, Co. } \\
\text { "Surplus" case }\end{array}$ & $\begin{array}{l}\text { Clay Loam } \\
\text { Loam } \\
\text { Sandy Loam }\end{array}$ & $\begin{array}{l}76 \\
75 \\
64\end{array}$ & $\begin{array}{l}9.79 \\
11.0 \\
10.1\end{array}$ \\
\hline $\begin{array}{l}\text { Denver, Co. } \\
\text { "Deficit" case }\end{array}$ & $\begin{array}{l}\text { Clay Loam } \\
\text { Loam } \\
\text { Sandy Loam }\end{array}$ & $\begin{array}{l}81 \\
81 \\
81\end{array}$ & $\begin{array}{l}10.6 \\
10.7 \\
10.7\end{array}$ \\
\hline \multicolumn{4}{|c|}{ Chi-square critical values $\begin{aligned} x_{0.05,7}^{2} & =14.1 \\
x_{0.01,7}^{2} & =18.5\end{aligned}$} \\
\hline
\end{tabular}


TABLE VIII

COMPARISON OF PHYSICALLY BASED BETA DISTRIBUTION PARAMETERS WITH THOSE OBTA INED ON THE BASIS OF MONTE-CARLO SIMULATIONS OF THE "COMPLETE" MOISTURE MODEL (KOCH AND MTUNDU, 1986a)

\begin{tabular}{|c|c|c|c|c|c|}
\hline \multirow{3}{*}{ Location } & \multirow{3}{*}{ Type of Soil } & \multicolumn{4}{|c|}{ Beta-pdf Parameters } \\
\hline & & \multicolumn{2}{|c|}{$\alpha$} & \multicolumn{2}{|c|}{$\beta$} \\
\hline & & $\begin{array}{l}\text { Physically } \\
\text { Based }\end{array}$ & Simul ated & $\begin{array}{l}\text { Physically } \\
\text { Based }\end{array}$ & Simul ated \\
\hline $\begin{array}{l}\text { Bl acksburg: } \\
\text { "Surplus" case }\end{array}$ & $\begin{array}{l}\text { Clay Loam } \\
\text { Loam } \\
\text { Sandy Loam }\end{array}$ & $\begin{array}{l}1.70 \\
0.467 \\
0.148\end{array}$ & $\begin{array}{l}5.93 \\
2.56 \\
1.84\end{array}$ & $\begin{array}{l}2.37 \\
2.35 \\
3.75\end{array}$ & $\begin{array}{l}2.24 \\
2.75 \\
4.13\end{array}$ \\
\hline $\begin{array}{l}\text { Denver: } \\
\text { "Deficit" case }\end{array}$ & $\begin{array}{l}\text { Clay Loam } \\
\text { Loam } \\
\text { Sandy Loam }\end{array}$ & $\begin{array}{l}4.03 \\
3.33 \\
3.25\end{array}$ & $\begin{array}{l}3.72 \\
3.00 \\
2.73\end{array}$ & $\begin{array}{l}13.63 \\
10.65 \\
10.38\end{array}$ & $\begin{array}{l}22.82 \\
17.20 \\
15.41\end{array}$ \\
\hline
\end{tabular}


moments of the moisture process was derived on the basis of simplified assumptions aimed at maintaining the analytical expressions mathematically tractable. Thus, in the stochastic model, it was assumed that the ground surface becomes wet instantaneously and that the moisture redistribution process that follows immediately after a rainfall and infiltration event also occurs instantaneously. The so-called "complete" model used to simulate the moisture process, of course, accounts for the finite durations of these "wetting" and "redistribution" processes.

In order to demonstrate the impact of these assumptions, Koch and Mtundu (1986b) also tested a simulation model that made the same "instantaneous wetting and redistribution" assumption. Using the mean and variance values obtained on the basis of this model, the beta-pdf parameters are compared with the physically based values in Table IX. These results indicate that, whereas some improvement is gained in some of the values, it is not in others. Overall, though, there is better agreement in this case.

The second cause for the discrepancy between physically based and simulated parameter values may be statistical error. This was evidenced by fluctuating parameter values as the sample size used to calculate the mean and variance was steadily increased from 100 to 1000 , al though such fluctuations are normally expected to die out with larger sample sizes.

One also notes at this juncture that the moments of cumulative infiltration (E[U] and $E\left[U^{2}\right]$ ) which appear in the stochastic model (see Eqs. 8.30 to 8.33 ) are functions of a constant averaged value of initial moisture. This observation alone would appear to immediately render the 
COMPARISON OF PHYSICALLY BASED BETA DISTRIBUTION PARAMETERS WITH THOSE

OBTAINED ON THE BASIS OF MONTE-CARLO SIMIILATIONS OF THE

"INSTANTANEOUS WETTING AND REDISTRIBUTION" MODEL

(KOCH AND MTUNDU, 1986b)

\begin{tabular}{|l|l|c|c|c|c|}
\hline \multirow{2}{*}{ Location } & \multirow{2}{*}{ Type of Soil } & \multicolumn{4}{|c|}{ Beta-pdf parameters } \\
\cline { 3 - 6 } & & $\begin{array}{l}\text { Physicallyy } \\
\text { Based }\end{array}$ & Simulated & $\begin{array}{l}\text { Physically } \\
\text { Based }\end{array}$ & Simul ated \\
\cline { 3 - 6 } & & 1.53 & 3.27 & 1.83 & 3.39 \\
\hline $\begin{array}{l}\text { Bl acksburg: } \\
\text { "Surplus" case }\end{array}$ & Clay Loam & 0.396 & 0.799 & 2.41 & 4.06 \\
& Loam & 0.147 & 1.333 & 3.75 & 6.40 \\
\hline \multirow{2}{*}{$\begin{array}{l}\text { Denver: } \\
\text { "Deficit" case }\end{array}$} & Clay Loam & 4.02 & 3.39 & 13.6 & 18.8 \\
& Loam & 3.33 & 3.03 & 10.7 & 15.8 \\
& Sandy Loam & 3.25 & 2.99 & 10.4 & 15.6 \\
\hline
\end{tabular}


model invalid. However, as observed in Chapter VII, these moments are practically invariant over the whole range of possible initial moisture values except for the region near saturation. Once they are obtained, the moments may well be taken to be independent of initial soil moisture except in humid regions where the soil is persistently saturated over long periods of time. Although the results are not presented here, there was practically no difference between the mean and variance values obtained via a simulation model in which the infiltration submodel was based on a variable initial moisture and those in which the infiltration was calculated on the basis of an invariant initial soil moisture.

For completeness, the simulated moment properties are compared with those based on the stochastic model (Eqs 8.12, 8.13, and 8.14 for the "deficit" case and Eqs. 8.17, 8.18 and 8.20 for the "surplus" case) in Table $x$. As can be observed in this table, the stochastic model values closely agree with those based on simulations of the "instantaneous wetting and redistribution" model; this agreement is due to the similarity in the assumptions inherent in these models. The stochastic model failed to predict the autocorrelation coefficient of initial moisture for clay loam in the "surplus" case due to the rather slow draining property of this soil as reflected in its low saturated hydraulic conductivity; the tendency to be near saturation most of the time, therefore, renders the model invalid in this case. 
TABLE 111.

COMPARISON OF THE RESULTS FROM THE SIMULATION OF THE PHYSICAL SOIL MDISTURE OYNAMIC MODEL WITH THOSE FROM THE STOCHASTIC SOIL MOISTURE MODEL

\begin{tabular}{|c|c|c|c|c|c|c|c|c|}
\hline \multirow{2}{*}{$\begin{array}{l}\text { Location and } \\
\text { Case }\end{array}$} & \multirow{2}{*}{$\begin{array}{l}\text { Type of } \\
\text { Soil }\end{array}$} & \multirow{2}{*}{$\begin{array}{l}\text { Stochastic } \\
\text { Property }\end{array}$} & \multicolumn{2}{|c|}{ Complete Model } & \multicolumn{2}{|c|}{$\begin{array}{l}\text { Inst ant aneous } \\
\text { "vett ing" model }\end{array}$} & \multicolumn{2}{|c|}{$\begin{array}{l}\text { instantaneous "wett ing" and } \\
\text { "redistribution" model }\end{array}$} \\
\hline & & & Physical & stocnastic & Physical & Stochastic & Physical & Stochastic \\
\hline Bl acksburg, Va & Clay loam & $E[\theta]$ & 0.459 & 0.431 & 0.452 & 0.432 & 0.435 & 0.437 \\
\hline \multirow[t]{5}{*}{ "Surpl us" } & & Std. Dev. $\{0\}$ & 0.0202 & 0.0291 & 0.0193 & 0.0295 & 0.0268 & 0.0317 \\
\hline & Loam & $E[\theta]$ & 0.377 & 0.335 & 0.368 & 0.335 & 0.337 & 0.335 \\
\hline & & Std. Dev. $[\theta]$ & 0.0338 & 0.027 & 0.0288 & 0.0277 & 0.0317 & 0.0283 \\
\hline & Sandy loam & $E[\theta]$ & 0.265 & 0.220 & 0.253 & 0.220 & 0.221 & 0.220 \\
\hline & & 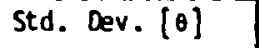 & 0.0412 & 0.0194 & 0.0330 & 0.0194 & 0.0235 & 0.0194 \\
\hline Denver. $C o$. & Clay loam & $E[\theta]$ & 0.449 & 0.370 & 0.442 & 0.371 & 0.349 & 0.378 \\
\hline \multirow[t]{5}{*}{ "Surplus" } & & Std. Dev. [0] & 0.0249 & 0.0228 & 0.0237 & 0.0230 & 0.0375 & 0.0272 \\
\hline & Loan & $E[0]$ & 0.362 & 0.315 & 0.350 & 0.315 & 0.291 & 0.315 \\
\hline & & Std. Dev. $[\theta]$ & 0.0464 & 0.0193 & 0.0451 & 0.0193 & 0.0361 & 0.0196 \\
\hline & Sandy loam & $E[\theta]$ & 0.247 & 0.212 & 0.234 & 0.212 & 0.182 & 0.212 \\
\hline & & Std. Dev. [0] & 0.0486 & 0.0117 & 0.0447 & 0.0117 & 0.0291 & 0.0117 \\
\hline Denver, Co. & $\mathrm{Clay}$ loam & $E[\theta]$ & 0.239 & 0.246 & 0.238 & 0.246 & 0.238 & 0.246 \\
\hline \multirow[t]{5}{*}{ "Deficit" } & & Std. Dev. $[\theta]$ & 0.0158 & 0.0155 & 0.0155 & 0.0156 & 0.0155 & 0.0156 \\
\hline & Loam & $E[\theta]$ & 0.182 & 0.188 & 0.181 & 0.188 & 0.181 & 0.188 \\
\hline & & Std. Dev. $[\theta]$ & 0.0194 & 0.0176 & 0.0190 & 0.0176 & 0.0190 & 0.0176 \\
\hline & Sandy loam & $E[\theta]$ & 0.0821 & 0.0882 & 0.0812 & 0.0882 & 0.0812 & 0.0882 \\
\hline & & Std. Dev. $\{\theta\}$ & 0.0194 & 0.0178 & 0.0190 & 0.0178 & 0.0190 & 0.0178 \\
\hline
\end{tabular}


CHAPTER IX

APPLICATION OF THE INITIAL MOISTURE PROBABILITY OENSITY FUNCTION

\section{INTRODUCTION}

As indicated previously, a significant factor in all efforts to develop physically based stochastic models of hydrologic processes is the apparent dependence of such processes on initial soil moisture. Evidence of such sensitivity has been demonstrated in previous chapters as well as by a number of investigators as noted previously. In particular, Koch (1982) derived probability distributions and moments of such hydrologic processes as cumulative infiltration, excess precipitation, recharge to the groundwater system and streamflow based on an initial soil moisture value that was, in effect, treated as fixed parameter. In other words, the derived distributions and moments were, essentially, conditional on initial soil moisture. Given the postulated probability distribution of initial soil moisture (in the preceding chapter), the effect of using an effective value, such as the mean, of initial moisture in calculating the moments of the hydrologic processes can now be investigated. This investigation is carried out by comparing the unconditional moments obtained by integrating the conditional moments over the entire possible range of initial moisture values as indicated by Eq. 2.1, with those obtained based on treating the initial soil moisture as a fixed parameter or effective value. 
MOMENTS OF CUMULATIVE INFILTRATION

The moments of cumulative infiltration, as derived by Koch (1982), have already been given (Eqs. 5.21 and 5.22). Application of Eq. 2.1 to these conditional moments gives the following unconditional moments:

$$
\begin{aligned}
& E[U]=1 /(\lambda \beta)-\beta \int_{\theta_{1}}^{\theta_{U}} \int_{K_{S}}^{\infty} f_{1}(r) g\left(\theta_{i}\right) d r d \theta_{i} \\
& E\left[U^{2}\right]=4 /(\lambda \beta)^{2}-\beta \int_{\theta_{1}}^{\theta_{u}} \int_{K_{S}}^{\infty} f_{2}(r) g\left(\theta_{i}\right) d r d \theta_{i}
\end{aligned}
$$

in which $f_{1}(r)$ is given by the integrand in Eq. 5.21, $f_{2}(r)$ is given by the integrand in Eq. 5.22, and $g\left(\theta_{j}\right)$ is given by Eq. 8.26 for the "surplus" case and by Eq. 8.27 for the "deficit" case. The lower and upper limits, $\theta_{1}$ and $\theta_{u}$, respectively, for the outer integral taken with respect to $\theta_{i}$ correspond to $\theta_{r}$ and $\theta_{s}$, respectively, for the "surplus" case and to $\theta_{w p}$ and $\theta_{r}$, respectively, for the "deficit" case.

The complex expressions given in Eqs. 9.1 and 9.2 cannot be simplified any further and, therefore, can only be evaluated numerically. Based on the characteristics of the two climates described in Chapter VI and given in Table I and on the properties of the three soils given in Table II, the moments of cumulative infiltration based on the mean value of initial soil moisture and those "weighted" by the 
probability density function of initial moisture were computed. The results are displayed in Table XI. As can be observed from this table, for each of the two "surplus" cases, the unconditional moments are approximately equal to the moments computed on the basis of the mean value of initial soil moisture for all the soil types except clay loam, for which there are some discernible differences in value. For the "deficit" case, there is practically no difference between the moments obtained by the two methods. These results are not too surprising in view of the apparent insensitivity of cumulative infiltration to initial soil moisture for the more permeable soils (loam and sandy loam) as was observed in Figs. 21 and 22, whereas the dependence of cumulative infiltration on initial soil moisture was observable for the less permeable clay loam soil.

\section{MOMENTS OF PRECIPITATION EXCESS}

The first and second conditional moments of precipitation excess, as derived by Koch (1982), were presented as follows:

$$
\begin{aligned}
E\left[V \mid \theta_{i}\right] & =\int_{K_{S}}^{\infty} B\left[\frac{r-K_{S}}{\lambda}-\sqrt{\frac{\pi}{\lambda}} \frac{S}{2} \exp \left(A^{2}\right) \operatorname{erfc}(A)\right] \exp (-B r-B) d r \\
E\left[V^{2} \mid \theta_{i}\right] & =\int_{K_{S}}^{\infty} B\left[\frac{2\left(r-K_{S}\right)^{2}}{\lambda^{2}}-\frac{S^{2}}{2 \lambda}\right. \\
& \left.-\sqrt{\frac{\pi}{\lambda}}\left(\frac{3 S\left(r-K_{S}\right)}{2 \lambda}-\frac{S^{3}}{8\left(r-K_{S}\right)}\right) \operatorname{erfc}(A) \exp \left(A^{2}\right)\right] \exp (-B r-B) d r
\end{aligned}
$$


TABLE XI

MOMENTS OF CUMULATIVE INFILTRATION

\begin{tabular}{|c|c|c|c|c|c|}
\hline \multirow{2}{*}{$\begin{array}{l}\text { Location } \\
\text { and } \\
\text { Case }\end{array}$} & \multirow{2}{*}{$\begin{array}{l}\text { Type } \\
\text { of } \\
\text { So il }\end{array}$} & \multicolumn{2}{|c|}{$\begin{array}{c}\text { Based on Mean Initial } \\
\text { Soil Moisture }\end{array}$} & \multicolumn{2}{|c|}{$\begin{array}{l}\text { Unconditioned by Beta PDF } \\
\text { of Initial Soil Moisture }\end{array}$} \\
\hline & & $\begin{array}{l}E[U] \\
(\mathrm{cm})\end{array}$ & $\begin{array}{l}\mathrm{E}\left[\mathrm{U}^{2}\right] \\
\left(\mathrm{cm}^{2}\right)\end{array}$ & $\begin{array}{l}E[U] \\
(\mathrm{cm})\end{array}$ & $\begin{array}{l}E\left[\mathrm{U}^{2}\right] \\
\left(\mathrm{cm}^{2}\right)\end{array}$ \\
\hline $\begin{array}{l}\text { Bl acksburg } \\
\text { "surplus" }\end{array}$ & $\begin{array}{l}\text { Clay loam } \\
\text { Loam } \\
\text { Sandy loam }\end{array}$ & $\begin{array}{l}0.935 \\
1.45 \\
1.52\end{array}$ & $\begin{array}{l}1.97 \\
7.20 \\
9.25\end{array}$ & $\begin{array}{l}0.919 \\
1.45 \\
1.52\end{array}$ & $\begin{array}{l}1.92 \\
7.15 \\
9.25\end{array}$ \\
\hline $\begin{array}{l}\text { Denver } \\
\text { "surplus" }\end{array}$ & $\begin{array}{l}\text { Clay loam } \\
\text { Loam } \\
\text { Sandy loam }\end{array}$ & $\begin{array}{l}0.679 \\
0.888 \\
0.899\end{array}$ & $\begin{array}{l}1.17 \\
2.95 \\
3.23\end{array}$ & $\begin{array}{l}0.669 \\
0.884 \\
0.899\end{array}$ & $\begin{array}{l}1.14 \\
2.90 \\
3.23\end{array}$ \\
\hline $\begin{array}{l}\text { Denver } \\
\text { "deficit" }\end{array}$ & $\begin{array}{l}\text { Clay loam } \\
\text { Loam } \\
\text { Sandy loam }\end{array}$ & $\begin{array}{l}0.358 \\
0.374 \\
0.375\end{array}$ & $\begin{array}{l}0.426 \\
0.553 \\
0.561\end{array}$ & $\begin{array}{l}0.358 \\
0.374 \\
0.375\end{array}$ & $\begin{array}{l}0.427 \\
0.553 \\
0.561\end{array}$ \\
\hline
\end{tabular}


in which:

$$
A=\frac{S \gamma \lambda}{2\left(r-K_{S}\right)}, \quad B=\frac{\lambda S^{2}}{2 r\left(r-K_{S}\right)}
$$

and where $V$ is the precipitation excess and all other variables and coefficients are as defined previously. The unconditional moments are obtained as before as follows:

$$
\begin{aligned}
& E[V]=\beta \int_{\theta_{1}}^{\theta_{u}} \int_{K_{S}}^{\infty} h_{1}(r) g\left(\theta_{j}\right) d r d \theta_{i} \\
& E[V]=\beta \int_{\theta_{1}}^{\theta_{1}} \int_{K_{S}}^{\infty} h_{2}(r) g\left(\theta_{i}\right) d r d \theta_{i}
\end{aligned}
$$

in which $h_{1}(r)$ represents the integrand in Eq. 9.3 and $h_{2}(r)$ represents the integrand in Eq. 9.4 and $g\left(\theta_{j}\right)$ together with the lower and upper limits are as described in the previous section. Numerical integration of these expressions resulted in the values summarized in Table XII. Similar to the cumulative infiltration results, the unconditional moments are, again, observed to approximately equal those based on the use of mean initial moisture as the effective value except for those corresponding to clay loam soil. This result is to be expected, of course, inasmuch as the two processes of cumulative infiltration and excess precipitation are complementary; that is: 
TABLE XII

MOMENTS OF EXCESS PRECIPITATION

\begin{tabular}{|c|c|c|c|c|c|}
\hline \multirow{2}{*}{$\begin{array}{l}\text { Location } \\
\text { and } \\
\text { Case }\end{array}$} & \multirow{2}{*}{$\begin{array}{l}\text { Type } \\
\text { of } \\
\text { So il }\end{array}$} & \multicolumn{2}{|c|}{$\begin{array}{l}\text { Based on Initial } \\
\text { Soil Moisture }\end{array}$} & \multicolumn{2}{|c|}{$\begin{array}{l}\text { Unconditioned by Beta PDF } \\
\text { of Initial Soil Moisture }\end{array}$} \\
\hline & & $\begin{array}{l}\mathrm{E}[\mathrm{V}] \\
(\mathrm{cm})\end{array}$ & $\begin{array}{l}E\left[V^{2}\right] \\
\left(\mathrm{cm}^{2}\right)\end{array}$ & $\begin{array}{l}E[V] \\
(\mathrm{cm})\end{array}$ & $\begin{array}{l}\mathrm{E}\left[\mathrm{V}^{2}\right] \\
\left(\mathrm{cm}^{2}\right)\end{array}$ \\
\hline $\begin{array}{l}\text { Bl acksburg } \\
\text { "surplus" }\end{array}$ & $\begin{array}{l}\text { Clay loam } \\
\text { Loam } \\
\text { Sandy loam }\end{array}$ & $\begin{array}{l}0.588 \\
0.0716 \\
0.000290\end{array}$ & $\begin{array}{l}3.90 \\
0.565 \\
0.0100\end{array}$ & $\begin{array}{l}0.604 \\
0.0756 \\
0.000340\end{array}$ & $\begin{array}{l}3.98 \\
0.586 \\
0.0105\end{array}$ \\
\hline $\begin{array}{l}\text { Denver } \\
\text { "surplus" }\end{array}$ & $\begin{array}{l}\text { Clay loam } \\
\text { Loam } \\
\text { Sandy loam }\end{array}$ & $\begin{array}{l}0.220 \\
0.0111 \\
5.96 \times 10^{-6}\end{array}$ & $\begin{array}{l}0.907 \\
0.0711 \\
3.18 \times 10^{-4}\end{array}$ & $\begin{array}{l}0.230 \\
0.0143 \\
9.98 \times 10^{-6}\end{array}$ & $\begin{array}{l}0.939 \\
0.0810 \\
4.09 \times 10^{-4}\end{array}$ \\
\hline $\begin{array}{l}\text { Denver } \\
\text { "deficit" }\end{array}$ & $\begin{array}{l}\text { Clay loam } \\
\text { Loam } \\
\text { Sandy loam }\end{array}$ & $\begin{array}{l}0.0168 \\
0.000432 \\
2.5 \times 10^{-8}\end{array}$ & $\begin{array}{l}0.0478 \\
0.00344 \\
2.0 \times 10^{-6}\end{array}$ & $\begin{array}{l}0.0162 \\
0.000401 \\
2.2 \times 10^{-8}\end{array}$ & $\begin{array}{l}0.0466 \\
0.00329 \\
1.8 \times 10^{-6}\end{array}$ \\
\hline
\end{tabular}




$$
E[V]=1 /(\lambda B)-E[U]
$$

where $1 /(\lambda \beta)$ represents the total rainfall depth. The relation between the second moments would be similar to Eq. 9.7 except for an additional term involving the expectation of the product of $U$ and $V$.

POTENTIAL EFFECT OF THE ASSUMPTION OF CONSTANT-INTENSITY STORMS

One of the expectations of this investigation was that the moments of such hydrologic processes as cumulative infiltration and excess precipitation would be under-or over-estimated when evaluated on the basis of an effective value of initial soil moisture. The results for clay loam soil have indicated this expectation as it has been observed that the moments of excess precipitation are underestimated whereas those of the corresponding cumulative infiltration are overestimated. The results for the more permeable soils, such as loam and sandy loam, have, however, indicated an apparent insensitivity to initial moisture. There is growing evidence that this apparent insensitivity may be attributed, at least in part, to the assumptions of constant-intensity rainstorms used throughout this study. For instance, Koch and Kekhia (1987) have shown that for low-permeability soils, such as clay loam, the constant-intensity assumption is adequate and that it does not lead to biased results in the prediction of excess precipitation; therefore, the effects of initial soil moisture would not be masked by this assumption. On the other hand, for the more permeable soils, such as loam and sandy loam, the same assumption leads to an 
under-prediction of excess precipitation. Therefore, the constantintensity assumption requires an evaluation in order to ascertain its impact on hydrologic response whenever the catchment soils have a high permeability. 
CHAPTER $X$

RELATION OF SOIL MOISTURE DYNAMICS TO THE OVERALL CATCHMENT RESPONSE: A DISCUSSION

\section{INTRODUCTION}

The scope of the present research did not include a study of how one would actually incorporate the stochastic behavior of soil water dynamics into the overall catchment response which, in essence, is represented by the streamflow from the catchment. This chapter treats this particular issue; however, the issue is addressed from a theoretical point of view in order to show that the research reported herein does not stand in apparent isolation. In particular, the purpose of this chapter is to show how events in the soil water zone would contribute to the overall response of the catchment modeled as a series of simple linear stochastic dynamical systems. In essence, the catchment is idealized as a transfer (or routing) model consisting of two linear storage reservoir series, namely, a surface reservoir sequence comprising the rapid-response subsystem and a subsurface reservoir sequence which constitutes the slow-response subsystem.

First, the deterministic model is presented in the form of a Nash-Dooge linear reservoir cascade (e.g. Bodo and Unny, 1985). The stochastic model is then formulated by randomizing the inputs to the determinstic model. The formulation results in a system of stochastic differential equations which represent a linear dynamical system. 
THE DETERMINISTIC CONTINUOUS-TIME RESERVOIR MODEL

A rigorous hydrodynamical model formulation of the hydrologic sytem represents a composite boundary-value problem whose feasibility is often limited by the infinite variety of boundary conditions present in the natural catchment. A level of conceptualization which closely approximates this hydrodynamical level is the systems representation of the catchment by a continuous-time storage reservoir. In this model, the continuity equation in the horizontal flow systems is replaced by a macroscopic or hydrologic version known as the water-balance for water-budget) equation wich represents one of the most fundamental concepts in hydrology. This is written as:

$$
P-E T-Q= \pm \Delta S
$$

where $P$ is the precipitation input to the catchment, ET represents the combined evaporation and transpiration losses, and $Q$ is the output, i.e. streamflow. All these quantities represent totals for some finite time $\Delta t$ for wich $\Delta S$ is the change in the water storage in the catchment. Considering Eq. 10.1 in the 1 imit as $\Delta t$ tends to zero yields the differential form of the continuity equation for the catchment, written as:

$$
\mathrm{ds} / \mathrm{dt}=\mathrm{x}-\mathrm{y}
$$

in which $x=P-E T$ and $y=Q$. In this definition, the inflow to the catchment is represented by the so-called effective precipitation. One 
could equally use the alternative approach where the catchment output is represented by $y=E T+Q$ and the input by $x=P$ (Bodo and Unny, 1985).

The dynamic equation in this systems-approach model is represented by a crude hydrologic version known as the linear storage reservoir defined by the relation:

$$
y=\alpha S, \quad \alpha>0
$$

or, alternatively,

$$
S=(1 / \alpha) y=k y
$$

where $\alpha$ is the reservoir coefficient and $k$ is known as the storage constant; the latter may be interpreted as the mean holding time of the reservoir.

The combination of Eqs 10.2 and 10.3 yields the following linear ordinary differrential equation (ODE) for a single linear reservoir:

$$
d y / d t+\alpha y=\alpha x
$$

The solution of Eq. 10.4 gives the reservoir outflow as a continuous function of time:

$$
y(t)=\int \alpha x(\tau) \exp [-\alpha(t-\tau)] d \tau+y_{0} \exp (-\alpha \tau)
$$

where $y_{0}$ is the outflow at time $t=t_{0}$. The kernel of this integral 
represents the unit impulse response function (irf) of a linear system (in this case the storage reservoir) which, in hydrological parlance, is referred to as the instantaneous unit hydrograph (IUH). The IUH is written as:

$$
h_{1}(t)=\alpha \exp (-\alpha t)=(1 / x) \exp (-t / k)
$$

where $h_{1}(t)$ is the solution of Eq. 10.4 for an instantaneous unit slug of input to a reservoir which is empty at $t$ ime $t=t_{0}$.

Thus far, one observes that the catchment has been represented as one unit, the storage reservoir, and its corresponding IUH is characterized by one parameter, $\kappa$. Obviously, this results in a fairly gross lumping of the governing parameters. Moreover, the threshold that exists in the soil water zone of the subsurface system shown in Fig. 4 (Chapter I), resulting in a delayed response of this system compared to that of the surface, makes it impossible to treat the whole catchment as linear. In order to ayoid these difficulties, the conceptualization suggested above is applied separately to each of the surface and subsurface components.

For the surface system, the input becomes the precipitation excess $V\left(P_{e}\right.$, in Fig. 4) with the direct runoff $\left(Q_{s}\right)$ being the output. Thus, the continuity equation for the surface system becomes:

$$
P-U-Q_{S}=\Delta S_{1}
$$

where $U$ is the infiltration and $\Delta S_{1}$ is the change in storage in the 
surface regime. For the subsurface system, the input is the recharge (R) and the output is the baseflow, $Q_{S}$, and the continuity equation is, therefore, given by:

$$
R-Q_{b}=\Delta S_{2}
$$

where $\Delta S_{2}$ is the change in storage in the subsurface regime. The recharge is governed by the equation:

$$
R=\Phi(U)-E T
$$

in which $\Phi(U)$ represents a function of the soil moisture dynamics which indicates how much of the infiltrated volume reaches the groundwater system, and ET is the evapotranspiration loss. In this formulation, the ET is assumed to all take place from the subsurface system. The dynamic equation for both flow regimes remains the same (Eq. 10.3).

Now, two IUH's are required to describe the response of the whole catchment, one for each of the surface and subsurface components, and each IUH is characterized by its own storage constant, $\kappa$. Unit hydrograph studies have, however, shown that the surface response of the catchment is better simulated not just by a single reservoir, but by a cascade of linear reservoirs, each having the same storage constant, $k$, and in which the additional reservoirs, starting from the second one, accepts inputs only from the reservoir immediately upstream. This concept is illustrated in Fig. 42, which also shows the resulting 


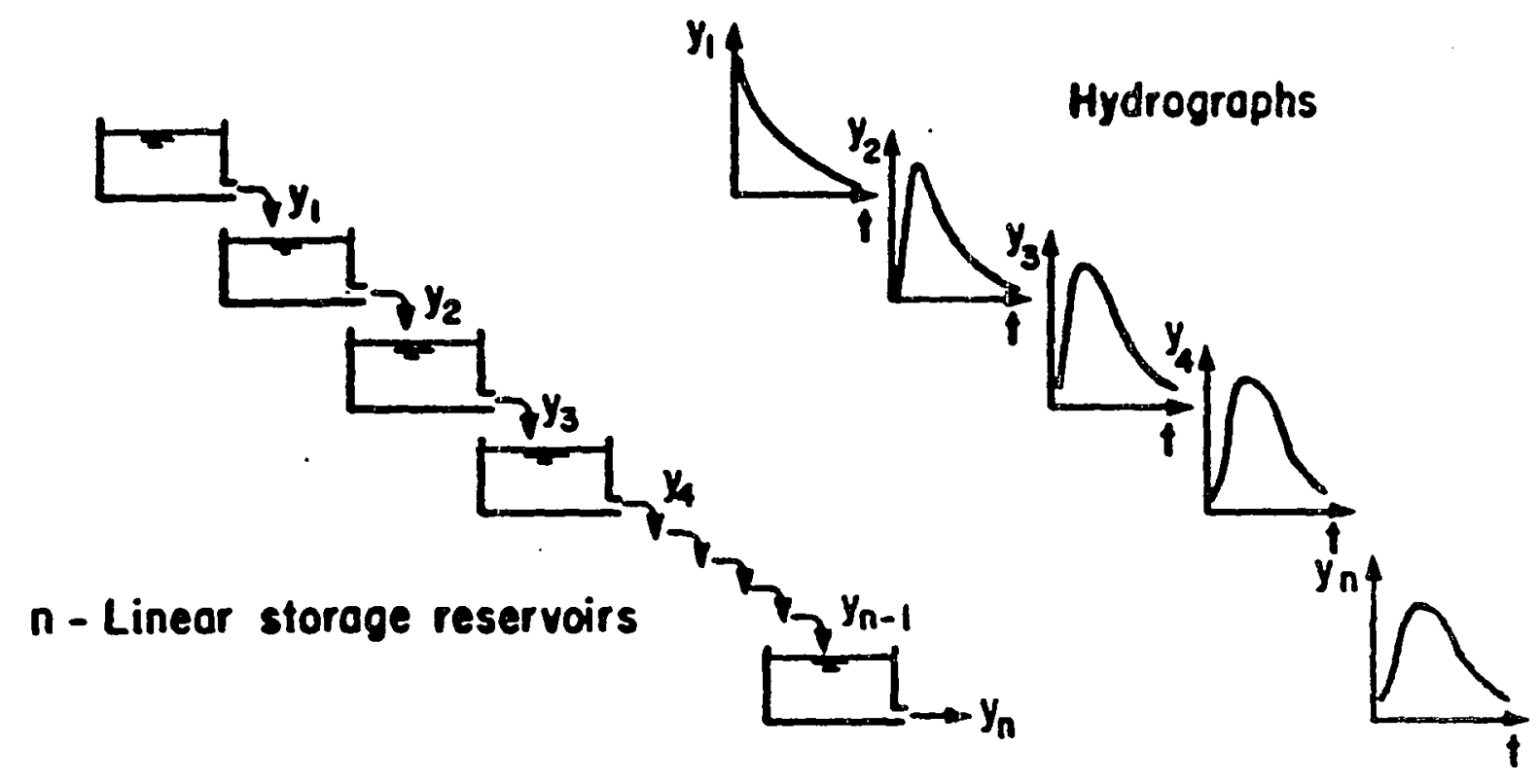

Figure 42. The linear reservoir cascade: (a) linear storage reservoirs; $(b)$ the corresponding hydrographs. 
hydrographs. In generalizing the result for a single reservoir to a cascade of reservoirs, Nash (1957) obtained the IUH of the nth reservoir as:

$$
\begin{aligned}
h_{n}(t) & =\left[\left(\alpha^{n} t^{n-1}\right) / \Gamma(n)\right] \exp (-\alpha t) \\
& =[1 / K \Gamma(n)](t / k)^{n-1} \exp (-t / k)
\end{aligned}
$$

where $\Gamma($.$) denotes the gamma function. Eq. 10.10$ mathematically represents the two-parameter gamma probability density function. With this generalization, the ODE for the single reservoir (Eq. 10.4) is replaced by the system of linear ODE's:

$$
\begin{aligned}
& d y_{1}=\left(\alpha x-\alpha y_{1}\right) d t \\
& d y_{2}=\left(\alpha y_{1}-\alpha y_{2}\right) d t \\
& \ldots \ldots \ldots \ldots \ldots \ldots \\
& \cdots \ldots \ldots \ldots \\
& d y_{n}=\left(\alpha y_{n-1}-\alpha y_{n}\right)
\end{aligned}
$$

where $x$ is the input to the first reservoir and $y_{i}$ is the output from the ith reservoir (for $1 \leqslant i \leqslant n$ ).

The reservoir-cascade concept can be applied to both the surface flow and subsurface flow regimes. Therefore, the parameters $k$ and $n$ appearing in the IUH (Eq. 10.10) have to be determined for each regime and can be related to the characterictics of the catchment. Nash (1957) 
determined these parameters by fitting the IUH to an empirically derived IUH using the method of moments. Other approaches have involved regression of some physical features of gauged catchments with the parameters of the IUH (e.g. Nash, 1960; Wu, 1963). Rosso (1984) proposed a method which parameterizes the IUH in terms of Horton order ratios of a catchment. This method is based on a geomorphologic model of catchment response. Various approaches, such as those cited above, can be assessed with the most appropriate one selected for continuing the analysis.

\section{SUMMARY OF THE DETERMINISTIC RESERVOIR MODEL}

The deterministic continuous-time reservoir model is summarized as follows. For the surface flow regime, the cascade of $n$ linear reservoirs may be represented by the system of ODE's:

$$
\begin{aligned}
& d y_{1}=\left[\alpha_{y}(P-U)-\alpha_{y} y_{1}\right] d t \\
& d y_{2}=\left(\alpha_{y} y_{1}-\alpha_{y} y_{2}\right) d t
\end{aligned}
$$

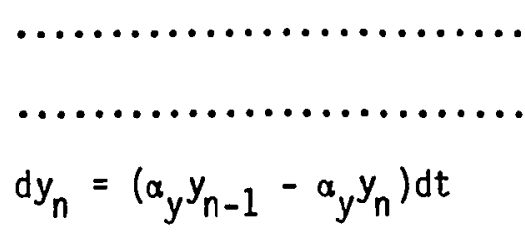

for which the IUH is given by:

$$
h_{n}(t)=\left[\alpha_{y}^{n} / \Gamma(n)\right] t^{n-1} \exp \left(-\alpha_{y} t\right)
$$


For the subsurface flow regime, a cascade of $\mathrm{m}$ linear reservoirs may be represented by the system of $00 E^{\prime} s$ :

$$
\begin{aligned}
& d z_{1}=\left(\alpha_{2} R-\alpha_{z} z_{1}\right) d t \\
& d z_{2}=\left(\alpha_{z^{2}} z_{1}-\alpha_{z^{2}} z_{2}\right) d t \\
& \text {................ } \\
& d z_{m}=\left(\alpha_{z} z_{m-1}-\alpha_{z} z_{m}\right) d t
\end{aligned}
$$

in which $R=\Psi(U)-E T$ and the IUH for the system is given by:

$$
h_{m}(t)=\left[\alpha_{z}^{m} / \Gamma(m)\right] t^{m-1} \exp \left(-\alpha_{z} t\right)
$$

The parameters $\alpha_{y}, \alpha_{z}, n$ and $m$ may be selected in such a way that the delayed response of the subsurface flow regime is incorporated, giving the total outflow from the whole catchment as:

$$
q(t)=y_{n}(t)+z_{m}(t)
$$

THE STOCHASTIC CONTINUOUS-TIME RESERVOIR MODEL

A stochastic conceptual model of the catchment can be obtained in at least three ways: either

(1) the catchment is described as a stochastic system driven by 
deterministic input processes; or

(2) the catchment behavior is considered deterministic and the input processes are randomized; or

(3) both the catchment parameters and the input processes are stochasticized.

There does not seem to be any examples of the first variant, but the second one appears to have been the most frequently used, especially in the study of stochastic outputs from storage reservoirs (e.g. Langbein, 1958; Jeng and Yevjevich, 1966; Klemes, 1973, 1974; Klemes and Boruvka, 1975; O'Connor, 1976; Bodo and Unny, 1985). The approach suggested herein would, to a great extent, involve the third variant which, in the context of reservoir-type of models, does not appear to have been utilized in the derivation of the stochastic structure of the output streamflow.

As noted previously, the main inputs to the catchment are precipitation and evapotranspiration, the latter being considered as a negative input. A stochastic representation of precipitation must characterize the interaction of rainfall with the physical environment through such essential features as the time between storms, the time distribution of storm intensity, and the storm depth. Various models have been proposed to describe precipitation with such characteristics (e.g. Grace and Eagleson, 1966; Gupta, 1973), but the one that has gained the most credence is the compound Poisson process (e.g Todorovic and Yevjevich, 1969; Eagleson, 1978b). This may be represented by an equation of the same form as Eq. 5.19: 


$$
P d t=\int_{0}^{\infty} v M(d t, d v)
$$

where $M(d t, d v)$ is a compound Poisson measure of arrival rate, $\lambda$, which is independent of the storm depth, $v$.

As observed in Chapter $V$, the process of evapotranspiration be modeled as a wide band noise with stationary zero-mean Gaussian fluctuations superposed on the mean rate (Eq. 5.23).

Upon substitution of these random functions (Eqs 10.17 and 5.23) into the two sets of ODE's given by Eqs 10.12 and 10.14, one obtains the stochasticized reservoir cascade model. For the surface system cascade, the first ODE (in Eq. 10.12) becomes:

$$
d y_{1}=\int_{0}^{\infty} \alpha_{y} v M(d t, d v)-\alpha_{y} U d t-\alpha_{y} y_{1} d t
$$

For the subsurface cascade, the first ODE is randomized into:

$$
d z_{1}=\alpha_{z} \Psi(U) d t-\left(\alpha_{z} \varepsilon+\alpha_{z} z_{1}\right) d t-\alpha_{z}^{\beta d W}
$$

In equations 10.18 and 10.19 , the processes $U d t$ and $\Psi(U) d t$, representing stochastic descriptions of the infiltration and redistribution processes, are dependent on the the stochastic properties of the soil hydraulic parameters.

The essential soil hydraulic parameters include $\theta_{r}, \theta_{S}, K_{S}$, 
$\psi_{w,}, B$ and $\eta$, as indicated in the constitutive relations of Eqs 3.6 and 3.7. There is mounting evidence that all these parameters may be described in terms of spatial stochastic structures (random fields) characterized by either normal or log-normal probability distributions (Freeze, 1975; Russo and Bresler, 1981). Introducing these parameters into the appropriate ODE's describing the processes of infiltration and redistribution would similary lead to a stochasticized model of the soil water system, from which the solution processes Udt and $\Psi(U) d t$ can be derived. Furthermore, a knowledge of the distribution of the hydraulic parameters in a given type of soil is required if the soil water dynamics are to be stochastically modeled in the absence of measured measured parameters. Predictive relationships which can be utilized in this regard have been developed by a number of investigators (e.g. Cosby et al, 1984). The relationships describe the hydraulic parameter distributions on the basis of the common descriptors of the physical properties of soil such as texture, structure and particle size distribution.

The above modeling procedure would enable a priori estimation of the soil moisture and streamflow processes together with their properties.

THE OUTPUTS OF THE STOCHASTIC CATCHMENT RESPONSE MODEL

The goal of relating soil moisture dynamics to catchment response is the determination of the statistical properties of the stochastic structure of the streamflow process, in terms of the joint statistical properties of the input processes, the physical characteristics of the 
soil and the catchment and the initial conditions. Achieving this goal means arriving at convenient solution representations of the systems of stochastic differential equations (SDE's), formulated as indicated above, from which properties such as the probability distribution functions as well as the mean, variance and autocovariance functions of the solution processes can be determined.

The solution of the SDE's can be accomplished by using either one of two main approaches, viz, the sample-function theoretic approach or the mean square theory. Because of its simplicity and because of the close parallel to the deterministic theory of ordinary differential equations, the mean square theoretic approach is the one upon which the analysis of stochastic problems in engineering heavily relies (Soong, 1973). The latter approach is the one that was used to analyze the problem of soil moisture in Chapter VI.

Under appropriate conditions, the systems of SDE's can be used to arrive at corresponding Fokker-Planck equations from which conditional (transition) and, hence, marginal probability distributions of the processes can be derived (Soong, 1973). Sample functions, which can be considered as representations of the streamflow process, can also be derived by numerical integration of the corresponding SDE systems. The moments of the processes, from which one obtains the mean, variance and covariance functions, can be derived either directly from the probability distributions or through the Ito transformation rule. 
CHAPTER XI

SUMMARY, CONCLUSIONS AND RECOMMENDATIONS

SUMMARY

Current efforts aimed at investigating models of catchment response, such as streamflow, have as one of their purposes the derivation of a physically based stochastic model of the watershed or catchment system in a manner which reflects the stochastic nature of the parameters of the physical system and the randomness of the climatic input processes. The goal of this line of research is two-fold. First, the cogritive aim involves efforts to understand better the significance of the physical environment on the stochastic nature of hydrologic processes, particularly streamflow. The second aim involves the application of such a model to prediction of catchment response, including the stochastic properties of the response, such as the mean, variance and autocorrelation functions, for the purpose of water resources planning and development.

Recent studies have, however, indicated that a potentially limiting factor in deriving physically based models is the dependence of hydrologic response on initial soil moisture conditions. Initial conditions in these studies were represented by an effective value of initial soil moisture or the initial moisture was treated simply as a parameter. Inasmuch as the dependence on initial moisture affects the 
distributions and moments of the hydrologic processes being investigated, there is a need to investigate the stochastic nature of soil moisture and, in particular, the soil moisture at the beginning of a storm event.

The present study, therefore, has focused on the stochastic behavior of soil moisture dymamics which, in light of the above, represents a very important component in the overall spectrum of processes that constitute the hydrological cycle. The significance of soil mnisture dynamics stems from the fact that the processes associated with soil water, such as infiltration, redistribution, drainage and evapotranspiration (ET), determine the amount and timing of water fluxes at the earth's surface. In particular, surface runoff to stream channels as well as subsurface flow to the groundwater system and eventually to the channel network are determined by soil moisture.

In order to study the soil moisture dynamics, a stochastic model has been developed in the form of a pair of stochastic differential equations (SDE's) of the Ito type. The sources of stochasticity in moisture dynamics have been linked to the stochastic driving inputs of infiltration (directly related to a Poisson-process description of rainfall events) and the energy-related evapotranspiration. One of the SDE's describes the "surplus" case, in which sufficient infiltration always occurs to allow for moisture depletion by the processes of drainage through and ET out of the root zone. The other SDE represents the "deficit" case, in which lack of adequate moisture leads only to an ET-controlled depletion process. The nature of the SDE's are such that they lead to sample functions involving Ito integral terms which can 
only be computed by numerical integration. However, analytical expressions are obtained for the moments, in particular the mean, variance and covariance.

A quantitative evaluation of the stochastic model has been undertaken in order to assess the potential for application as a predictive tool and to identify major limitations. The basis of this evaluation is a comparison of the results obtained from a Monte Carlo simulation using a simplified dynamic soil moisture model with those derived analytically from the stochastic model. The simplified deterministic model was presented by Koch et al (1986). The model simulates the prcesses of wetting, redistribution, drainage and evapotranspiration (Eqs. 5.11 to 5.15) which occur in the root zone of the soil. An evaluation of this model showed that it reasonably approximated observed field behavior at a point (see Figs. 5 and 6 , Chapter V). Therefore, this physical model provided a suitable tool for comparison with the stochastic model developed in this study.

From the general model of soil moisture, a specific model of initial soil moisture has been developed and its stochastic moments have been derived. Of particular interest is the probability distribution of initial soil moisture. Unfortunately, the complexity of the general soil moisture model and that of infiltration presently precludes its analytic derivation. Instead, an empirically fitted distribution has been postulated for the use in integrating the initial-moisturedependent hydrologic processes over the whole possible range of initial moisture. This distribution has permitted the assessment of how initial soil moisture affects the estimation of hydrologic response. 
A graphical summary of the model development and application is given by the block diagram in Fig. 43 and, in little more detail, by the diagrams in Figs. 44 to 47 . Included in the flow charts of Figs. 44 and 45 are the key parameters of the model as well as the key assumptions that were made at each stage of development.

\section{CONCLUSIONS}

Stochastic Model of Soil Moisture Dynamics

Application of the stochastic model of soil moisture developed herein requires the specification of both soil and climate properties. While most of the parameters may be obtained from statistical samples of the appropriate climate process or are physical properties of soil, the parameter $\beta^{2}$ related to the variability of ET presents some problems. The problems arise due to inadequate knowledge regarding the process and, clearly, the parametrization of the ET process requires further study. Alternatively, a different stochastic model of ET than that used in this study may be selected. In any event, with an appropriate

selection of the value of $B^{2}$, the present study has shown that physically realistic sample functions of the moisture evolution processs can be obtained for both the "surplus" and "deficit" cases.

The analytical nature of the moment equations derived from the sample functions shows explicity the interrelationship between the stochastic properties of soil moisture, namely the mean, variance and covariance, and the parameters of the physical and climatic systems. For example, the mean for both the "surplus" and "deficit" cases represents an expression for water balance in the root zone. Further, 


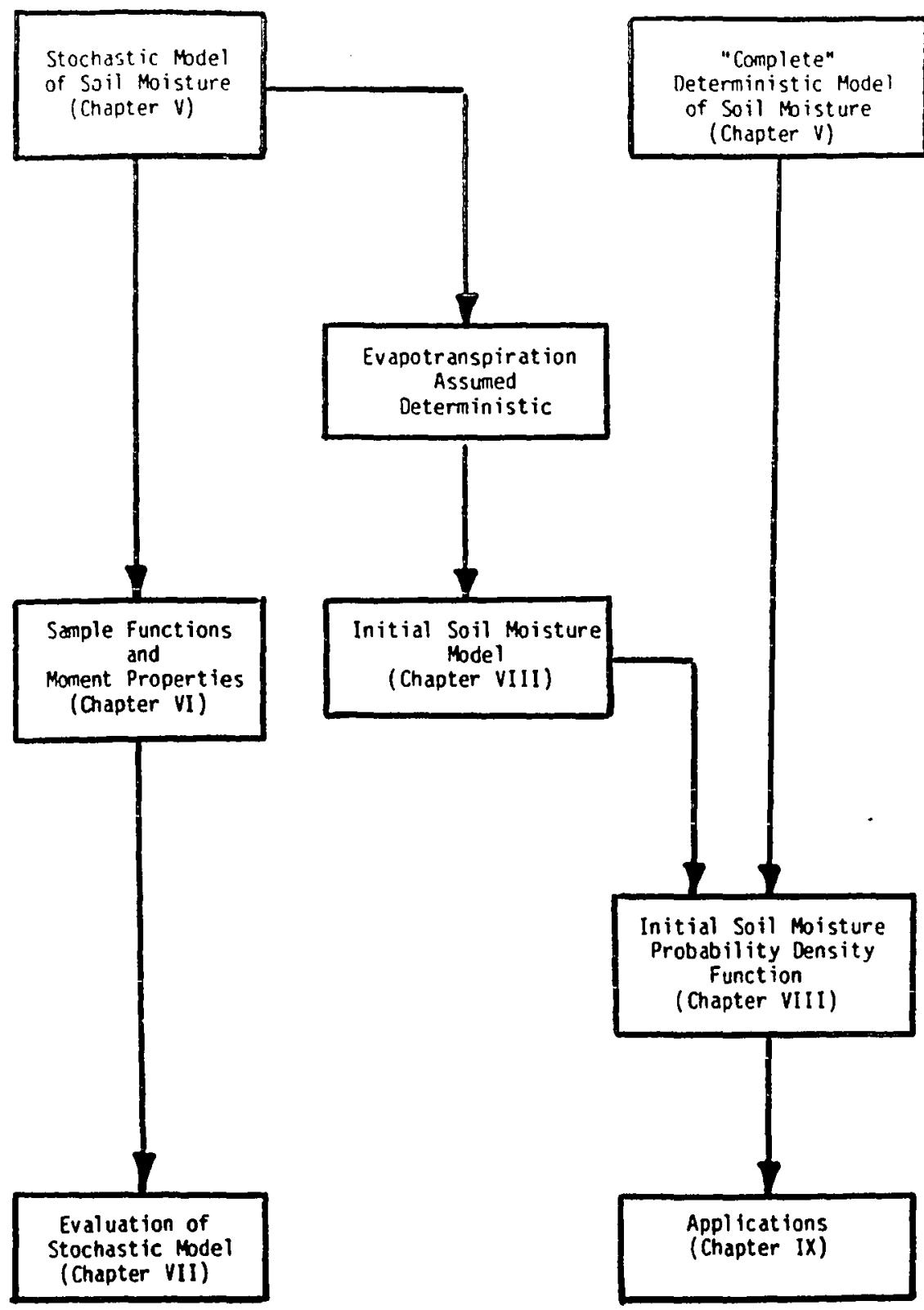

Figure 43. General summary of model development and application 


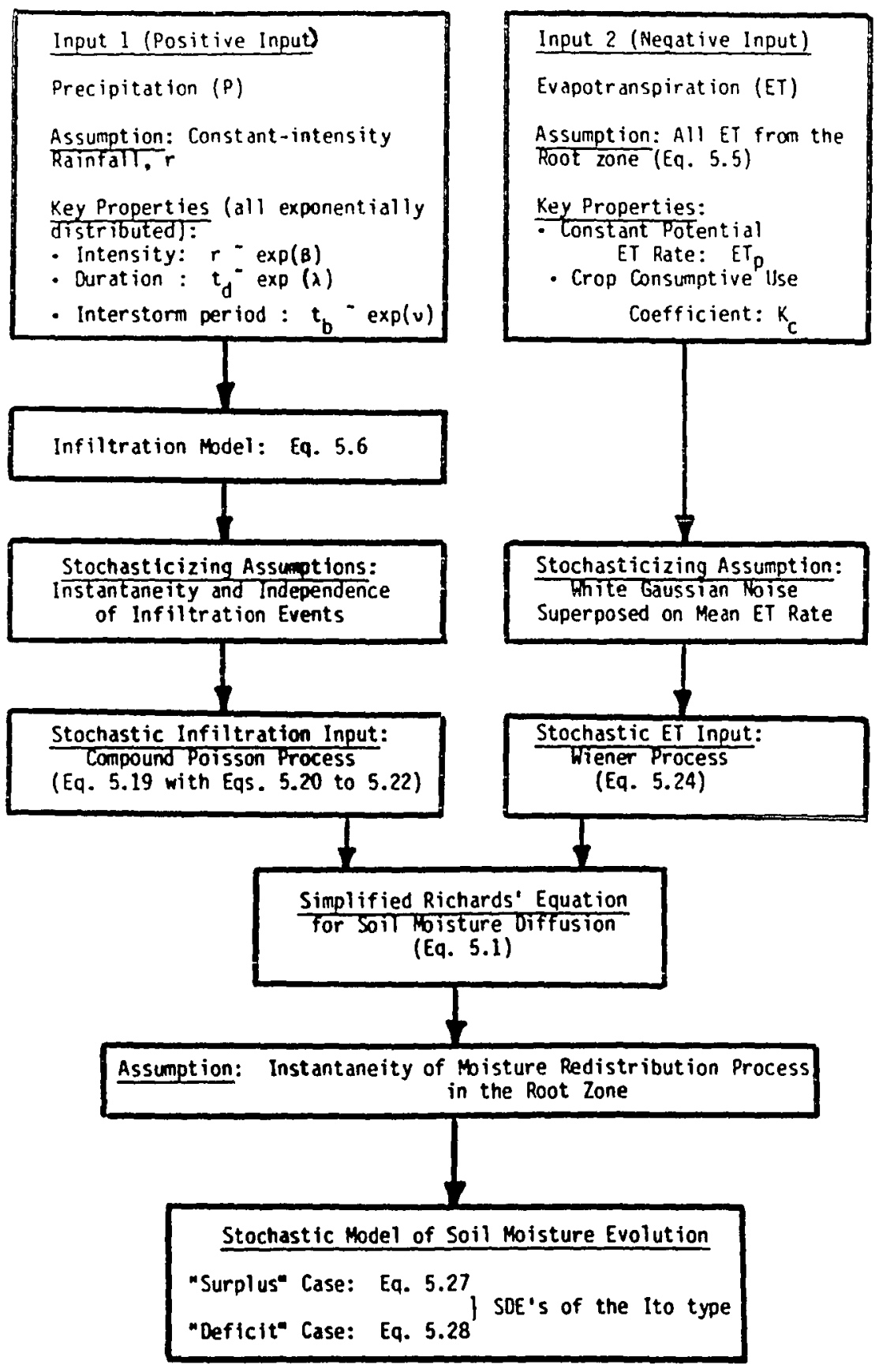

Figure 44. Symthesis of the stochastic model of soil moisture dymamics (Chapter V) 


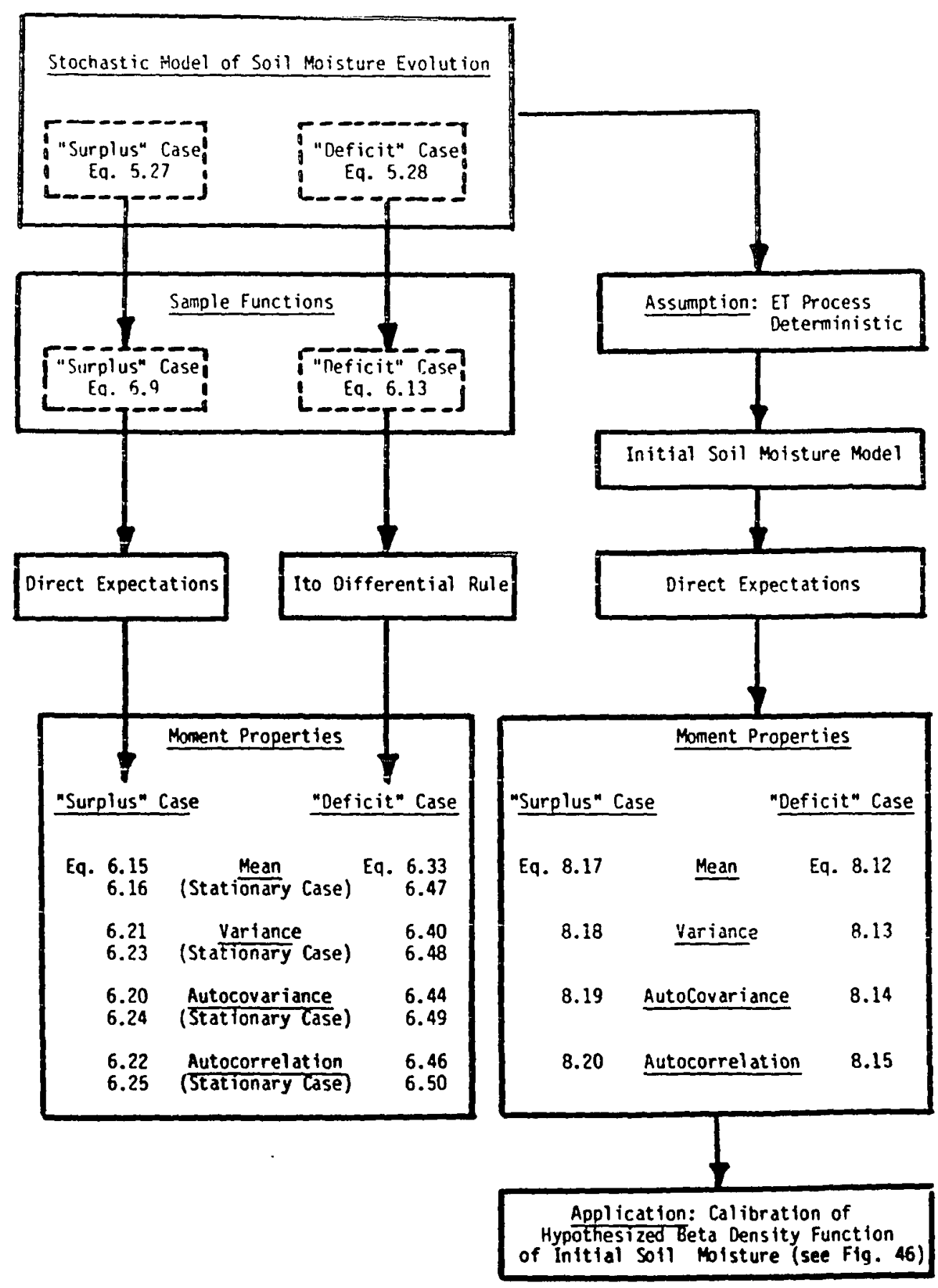

Figure 45. Analysis of the stochastic model of soil moisture TChapters VI and VIII) 


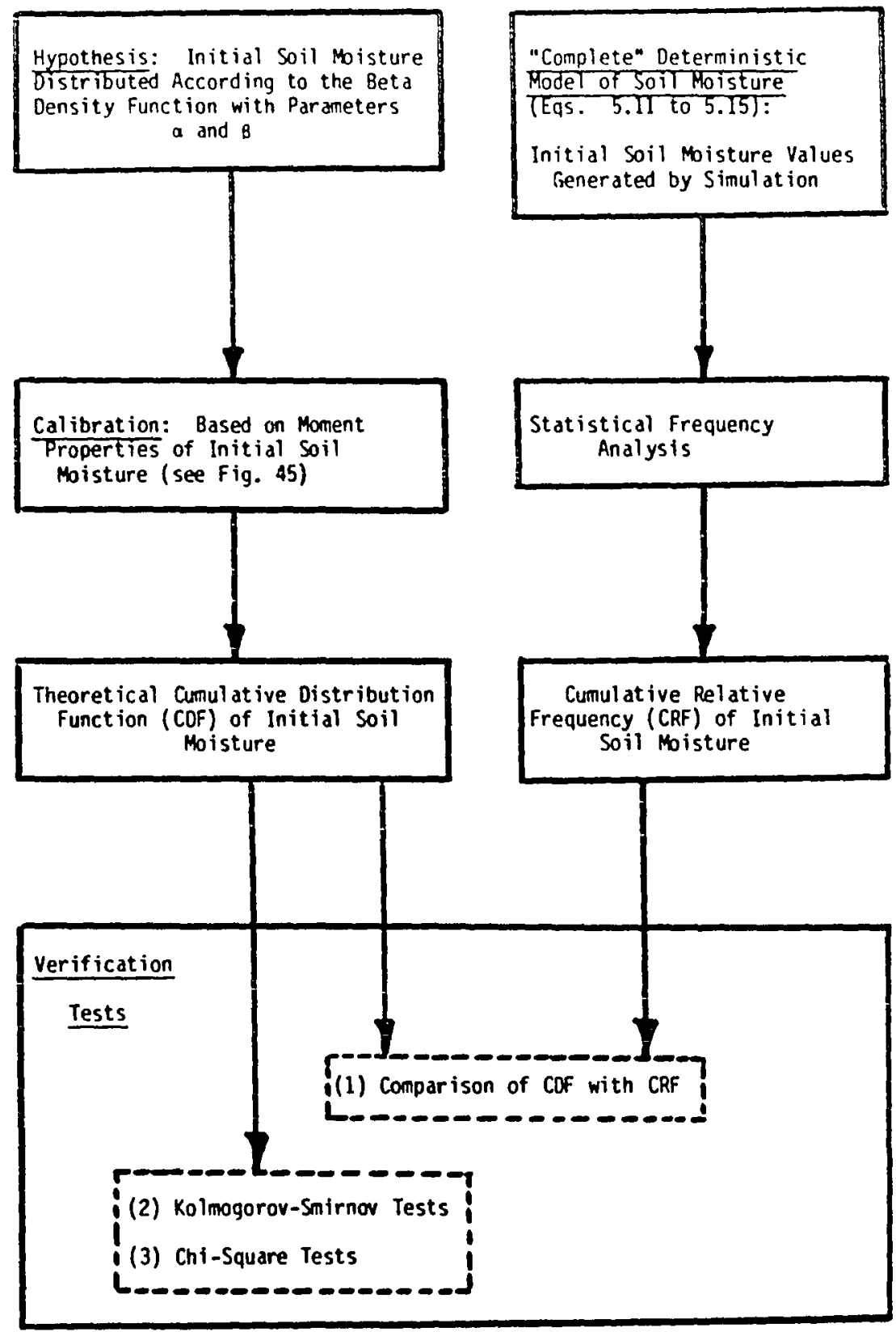

Figure 46. Statistical development of probability distribution of initial soil moisture (Chapter VIII) 


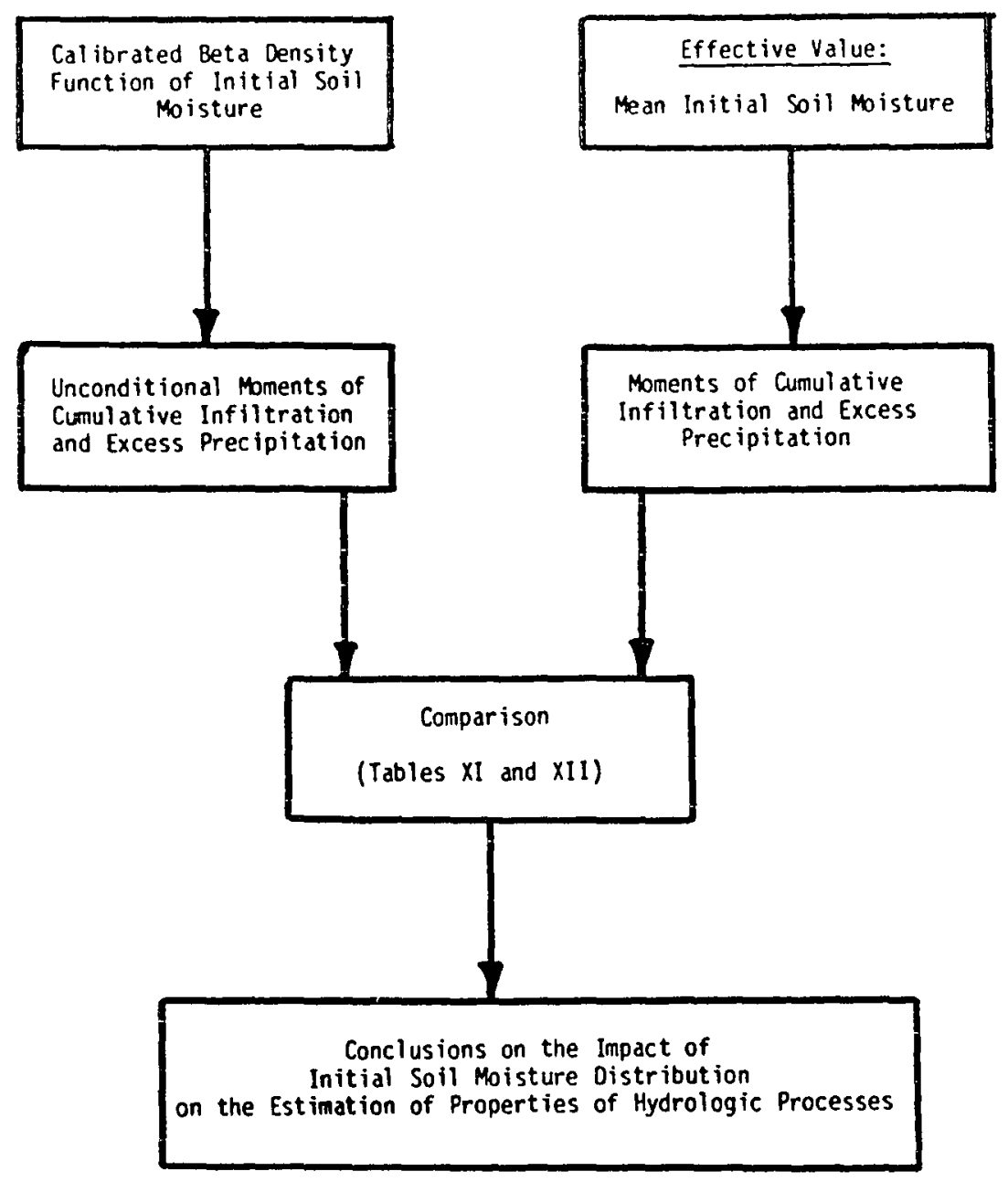

Figure 47. Estimation of moments of hydrologic processes (Chapter IX) 
the rate of decrease in the correlation function for the "surplus" case was controlled by the rate of drainage from the soil while in the "deficit" case, the correlation is directly related to the mean ET rate and available soil water content. Thus, the models have cognitive value and, with further development, may provide a vaiuable predictive tool.

The applicability of the stochastic model is, however, restricted to situations where either the "surplus" or "deficit" soil moisture conditions persist for the duration under consideration. Under the climatic conditions tested in this iudy, only the "deficit" case was found to be feasible for all soil types. The "surplus" state is evidently possible only under very wet climatic conditions, where there is very little ET and for less permeable soils like clay loam. All other climatic and soil conditions would produce a "transitory" behavior in which the soil moisture moves from the "surplus" to the "deficit" state and back.

In order to simplify the analytical derivation of the stochastic model, the processes of wetting and redistribution, which are accounted for in a physical dynamic model of soil moisture, were assumed to occur instantaneously. This assumption leads to a situation where the root zone attains a uniform soil water content immediately after every infiltration event. The major effect of "ignoring" these processes is a downward bias in the mean moisture and an underestimation of the variance especially for the more permeable soils under the "surplus" conditions. In the "deficit" case, lack of consideration of these processes has almost no effect on the mean moisture but produces a 
downward bias on the predicted variability of the moisture. Furthermore, this effect on variability seems to be enhanced by the use of a constant effective value of initial moisture in the stochastic model. Similar but opposite effects are evident in the autocorrelation structure of soil moisture. Whereas the autocorrelation function is biased upward by the simplifications in the "surplus" cases, it is essentially unaffected in the "deficit" cases.

In spite of some limitations in the application of the stochastic model, it evidently possesses sufficiently accurate behavior to justify its use in studying the stochastic behavior of soil moisture in a number of situations. In particular, the model has served as a useful tool in advancing the study to the problem of the random nature of initial moisture at a given point.

The stochastic model has been shown to be sensitive to initial conditions at the beginning of a storm as suggested by the obvious dependence of infiltration, the primary moisture input, on the initial value of moisture. The sensitivity to initial moisture is particulary marked for the less permeable soils, such as clay loam. Whereas the "deficit" case reveals almost no dependence on initial conditions for all soil types tested in this study, the "surplus" case exhibits some dependence, especially for less permeable soils and, further, this dependence is most pronounced when the soil is closer to saturation.

Use of the Stochastic Model to Estimate Hydrologic Response

One of the objectives of this research was to investigate the dependence of hydrologic response on the initial soil moisture, that is, the soil water content at the beginning of a rainstorm. This 
investigation has been carried out by unconditioning the moments of two such processes, namely cumulative infiltration and excess precipitation, based on a postulated distribution of initial soil moisture. The beta density function has been found to be a reasonable empirical probability distribution for initial soil moisture based on statistical tests.

In order to evaluate the impact of the dependence of hydrologic response on the distribution of initial soil moisture, the moments of two hydrologic processes were computed using, on the one hand, an average value of initial soil moisture as the effective value and, on the other hand, the beta distribution of initial moisture as the "weighting" function. The results of such computations showed that the distribution of initial moisture is not important in the estimation of the moments of hydrologic processes in areas where highly permeable soils, such as loam and sandy loam, prevail and, also, in environments where, regardless of the type of soil, the "deficit" state is maintained. In such cases, an effective value of initial soil moisture, in particular the mean, is sufficient in the estimation of hydrologic response.

The distribution of initial soil moisture, however, becomes important whenever the properties of hydrologic response have to be estimated under "surplus" conditions and, especially, if the soil has a low permeability (for example, clay loam). Although an effective value of initial soil moisture could still be used in such cases to obtain approximate values of the response properties, the estimates would be biased somewhat. For example, both the mean and the variance of cumulative infiltration would be overestimated whereas the mean and 
variance of excess precipitation, the complementary process, would be underestimated.

\section{RECOMMENDATIONS FOR FURTHER RESEARCH}

The stochastic model of soil moisture developed in this study has been evaluated on the basis of comparisons of its results with those obtained from a simulation of the deterministic model of soil moisture dynamics presented by Koch et al (1986). The latter model was, itself, empirically tested against field-monitored values of soil moisture as well as results from laboratory drainage experiments and reasonably good agreement was found. However, the model developed in the present study could also be empirically tested in a more direct manner than via simulations of a deterministic model. The tests could be conducted, for instance, at one of the many experimental watersheds under the management of the United States Department of Agriculture (USDA) (e.g. Claypan Experimental Watershed operated by the USDA-Agricultural Research Service and the University of Misssouri-Colombia near Kingdom City, Missouri).

In such experimental watersheds, the properties of soil(s) and the parameters related to the characteristics of the climate can easily be obtained. The rainfall and evapotranspiration charateristics are routinely monitored by well instrumented weather stations. Equipment for monitoring soil moisture in the field is also readily available.

For the purpose of testing the stochastic model developed herein, the soil moisture would be monitored over a period of, say, one month within a season in which the climatic properties are homogeneous. 
Rainfall and evapotranspiration (ET) characteristics would also be measured during this period. The amount of infiltration that occurs during each rainfall event would be estimated based on the measured values of runoff from the watershed. From all these measurements, the sample trajectory of soil moisture evolution could then be plotted and the statistical moments (mean, variance and autocorrelation) computed. Finally, the trajectory and the moments could be compared with the corresponding properties computed from the stochastic model based on the known characteristics of the climate and the properties of the soil(s) in the watershed.

The importance of initial soil moisture conditions at the beginning of a rainfall event in the estimation of hydrologic response in a catchment has been demonstrated in this investigation. Furthermore, as indicated in Chapter IX, there is growing evidence that the sensitivity of hydrologic response to initial soil moisture may be masked by the use of the assumption of constant-intensity rainstorms and that, therefore, the full impact of initial moisture conditions may not be appreciated as a result. Further research on this issue is clearly in order. For instance, the study could involve a re-working of the models presented here using variable-intensity rainstorms or approximating the variable-intensity storms by dividing the storm durations into shorter increments of constant-intensity as suggested by Koch and Kekhia (1987). Inasmuch as the hydrologic response is predicated on initial soil moisture, the recommended line of investigation is expected to reveal greater dependence of the distributions and moments of hydrologic processes on initial soil 
moisture.

Another important line of research that one might pursue involves the parametrization of the stochastic model of soil moisture dynamics derived in this investigation. The model has been, herein, evaluated on the basis of a deterministic evapotranspiration (ET) process because the stochastic model of ET used in this study requires the specification of the paramater $\beta^{2}$ related to the variability of ET. Due to inadequate knowledge of the ET process, an appropriate value of $\beta^{2}$ could not be selected. In view of this problem, either the parametrization of the ET model used here requires further study or a different model of ET needs to be be sought. In any event, the final model chosen would also require further assessment to prove its adequacy. A survey of the literature reveals that, while the ET process has been extensively studied (e.g. Brutsaert, 1982), its stochastic properties have not.

Finally, the presentation herein has included a chapter on how the soil moisture model might be fitted in the "big picture", that is, the catchment system as a whole. The "fitting" process has been carried out in a rather cursory manner and a proper study is required to demonstrate the entire process and, hence, complete the whole modeling procedure of stochastic catchment response based on a priori estimates of all submodel parameters.

The "fitting" process represents the next major aspect of catchment response modeling inasmuch as this leads to the aspect of the transfer mechanisms, that is, the routing of the rainfall excess through the surface flow system and of the the recharge through the subsurface (groundwater) system. Bodo and Unny (1985) have recently undertaken the 
modeling of this aspect within the formalism of stochastic differential equations (SDE's). An improvement in the Bodo-Unny approach in terms of physically based modeling may be accomplished by utilizing routing parameters which can be estimated a priori on the basis of significant characteristics of the catchment rather than resorting to statistical manipulation of available hydrologic data. The final step would then be to integrate the stochastic soil water model and the physically based routing parameters into the SDE-routing approach in order to arrive at an improved stochastic catchment response model. 


\section{BIBLIOGRAPHY}

Amorocho, J. and W.E. Hart. 1964. A Critique of Current Methods in Hydrologic Systems Investigations, Trans., Amer. Geophys. Union, 45(2), 307-321.

Bodo, B.A. and T.E. Unny. 1985. Linear Stochastic Conceptual Response Models for Small Catchments, Civil Engineering Department, Univ. of Waterloo, Waterloo, Canada.

Bodo, B.A., M.E. Thompson and T.E. Unny. 1985. Stochastic Differential Equations: A Primer, Civil Engineering Department, Univ. of Waterloo, Waterloo, Canada.

Boes, D.C. and J.D. Salas. 1978. Nonstationarity in the Mean and the Hurst Phenomenon, Water Resour. Res., 14(1), 135-143.

Box, G.E.P. and G.M. Jenkins. 1976). Time Series Analysis: Forecasting and Control, Holden-Day, $575 \mathrm{p}$.

Brutsaert, W. 1982. Evaporation into the Atmosphere: Theory, History, and Applications, D. Reidel Publishing Co., Dordrecht, Holland.

Cordova, J.R. and R.L. Bras. 1981. Physically Based Probalistic Models of Infiltration, Soil Moisture, and Actual Evapotranspiration, Water Resources Research, 17(1), pp. 93-106.

Cordova, J.R. and I. Rodriguez-Iturbe, 1985. On the Probabilistic Structure of Storm Surface Runoff, Water Resources Research, 21(5), pp. 755-763.

Corey, A.T. 1977. Mechanics of Heterogeneous Flow in Porous Media, Water Resour. Publications, Fort Collins, Colorado.

Cosby, B.J., G.M. Hornberger, R.B. Clapp, and T.R. Ginn. 1984. A Statistical Exploration of the Relationships of Soil Moisture Characteristics to the Physical Properties of Soils, Water Resour. Res., 20(6), 682-690.

Dagan, G. and E. Bresler. 1983. Unsaturated Flow in Spatially Variable Fields, 1. Derivation of Models of Infiltration and Redistribution, Water Resour. Res., 19(2), 413-420.

DeCoursey, D.G. 1982. Stochastic Models in Hydrology. In: Hydrologic Modeling of Small Watersheds, ed. by C.T. Haan, H.P. Johnson and D.L. Brakensiek, ASAE, 17-78. 
Diaz-Granados, M.A., J.B. Valdez and R.L. Bras. 1984. A Physically Based Flood Frequency Distribution, Water Resources Research, 20(7), pp. 995-1002.

Dooge, J.C.I. 1973. Linear Theory of Hydrologic Systems, Tech. Bull. No. 468, Agric. Res. Service, USDA, Washington, D.C.

Durbin, P.A. 1983. Stochastic Differential Equations and Turbulent Dispersion, NASA Reference Public. No. 1103, Washington, D.C.

Eagleson, P.S. 1971. The Stochastic Kinematic Wave. In: Systems Approach to Hydrology, Water Resour. Public., Fort. Collins, Colorado, 202-222.

Eagleson, P.S. 1972. Dynamics of Flood Frequency, Water Resour. Res., $8(4), 878-898$.

Eagleson, P.S. 1978a. Climate, Soil, and Vegetation 1. Introduction to Water Balance Dynamics, Water Resour. Res., 14(5), 705-712.

Eagleson, P.S. 1978b. Climate, Soil and Vegetation, 2. The Distribution of Annual Precipitation Derived from Observed Storm Sequences, Water Resour. Res., 14(5), 713-721.

Eagleson, P.S. 1978c. Climate, Soil and Vegetation, 3. A Simplified Model of Soil Moisture Movement in the Liquid Phase, Water Resour. Res., 14(5), 722-730.

Eag1:son, P.S. 1978d. Climate, Soil and Vegetation, 4. The Expected Value of Annual Evapotranspiration, Water Resour. Res., 14(5), $731-740$.

Eagleson, P.S. 1978e. Climate, Soil and Vegetation, 5. A Derived Distribution of Storm Surface Runoff, Water Resour. Res., 14(5),

Eagleson, P.S. 1978f. Climate, Soil and Vegetation, 6. Dynamics of the Annual Water Balance, Water Resour. Res., 14(5), 749-764.

Eagleson, P.S. 1978g. Climate, Soil and Vegetation, 7. A Derived Distribution of Annual Water Yield, Water Resour. Res., 14(5), 765-776.

Eagleson, P.S. 1982. Ecological Optimality in Water-Limited Natural Soil-Vegetation Systems, 1. Theory and Hypothesis, Water Resources Research, 18 (6), pp. 325-340.

Fiering, M.B. 1967. Streamflow Synthesis, Harvard Univ. Press, Cambridge, Mass., 135 p.

Freeze, R.A. 1974. Streamflow Generation, Rev. of Geophys. and Space Phys., 12(4), 627-647. 
Freeze, R.A. 1975. A Stochastic-Conceptual Analysis of One-Dimensional Flow in Non-uniform Homogeneous Media, Water Resour. Res., Ii(5), 725-741.

Freeze, R.A. 1980. A Stochastic-Conceptual Analysis of Rainfall-Runoff Processes on a Hillslope, Water Resour. Res., 16(2), 391-408.

Gihman, I.I. and A.V. Skorohod. 1972). Stochastic Differential Equations, Springer-Verlag, N.Y.

Grace, R.A. and P.S. Eagleson. 1966. The Synthesis of Short-time Increment Rainfall Sequences, Hydrodyn. Lab. Rep. 91, Dept of Civil Eng., MIT, Cambridge, Mass. (Original not seen; cited in Eagleson, j.978b).

Green W.H. and G. Ampt. 1911. Studies of Soil Physics, Part 1 - The Flow of Air and Water Through Soils, Jour. of Agicultural Sci., 4, 1-24.

Gupta, V.K. 1973. A Stochastic Approach to Space-time Modeling of Rainfal1, Tech. Rep. Natur. Reseur. Syst. 18, Univ. of Arizona, Tuscon, Arizona (Original not seen; cited in Eagleson, 1978b).

Hahn G.J. and S.S. Shapiro. 1967. Statistical Models in Engineering, John Wiley and Sons, Inc., N.Y.

Hipel, K.W., A.I. McLeod and W.C. Lennox. 1977. Advances in Box-Jenkins Modeling, 1. Model Construction, Water Resour. Res., $13(3), 567-575$.

Hoe1, P.G., S.C. Port and C.J. Stone. 1972. Introduction to Stochastic Processes, Houghton Miffl in Co., Boston.

Ito, K. 1951. On Stochastic Differential Equations, Mem. Am. Math. Soc., 4, 1-51.

Jeng, R.I. and V.M. Yevjevich. 1966. Stochastic Properties of Lake Outflows, Hydrol. Pap. 14, Colorado State Univ., Ft.Collins, Co.

Klemes, V. 1973. Watershed as Semi-infinite Storage Reservoir, Jour. Irrig. Orain. Div., Proc., ASCE, 99(R4), 477-491.

Klemes, V. 1974. Probability Distribution of Outflow from a Linear Reservoir, Jour. of Hydrol., 21, 305-314.

KTemes, V. 1978. Physically Based Stochastic Hydrologic Analysis. In: Advances in Hydroscience, Vol 11, ed. by V.T. Chow, Academic Press, 285-356.

Klemes, V. and L. Boruvka. 1975. Output from a Cascade of Discrete Linear Reservoirs with Stochastic Input, Jour. of Hydrol., 27, 1-13. 
Koch, R.W. (1982), A Physical-Probabilistic Approach to Stochastic Hydrology, Ph.D. Dissertation, Coiorado State univ., For $i$ Collins, Colo.

Koch, R.W. 1985. A Stochastic Streamflow Model Based on Physical Principles, Water Resour. Res., 21(4), 545-553.

Koch, R.W., R.L. Allen and N.D. Mtundu. 1986. Evaluation of a Simple, Dynamic So il Moisture Model, accepted for publication in the Journal of Irrigation and Drainage Engineering, ASCE.

Koch, R.W. and N.D. Mtundu. 1986a. On the Stochastic Nature of Soi1 Moisture, 1. Derivation of a Physically Based Model, submitted for review to Water Resources Research.

Koch, R.W. and N.D. Mtundu. 1986b. On the Stochastic Nature of Soil Moisture, 2. Evaluation of the Model Behavior, submitted for review to Water Resources Research.

Koch, R.W. and M.F. Kekhia. 1987. Effect of Rainfall Intensity Distribution on Excess Precipitation, to be Published in Proc. Engineering Hydrology Symposium, ASCE, Williamsburg, Virginia., Aug. 3-7, 1987.

Langbein, W.B. 1958. Queuing Theory and Water Storage, Jour. Hydraul. Div., Proc., ASCE, 84(HY5), 1811-1 to 1811-24.

Linsley, R.K. and N.H. Crawford. 1960. Computation of a Synthetic Streamflow Record on a Digital Computer, Intern. Assoc. Sicent if. Hydrol., Public. 51, 526-538.

Mandelbrot, B.B. and J.R. Wallis. 1968. Noah, Joseph and Operational Hydrology, Water Resour. Res., 4(5), 909-918.

Mein, R.G. and C.L. Larson. 1973. Modeling Infiltration During a Steady Rain, Water Resour. Res., 9(2), 384-394.

Mejia, J.M., I. Rodriguez-Iturbe, and D.R. Dawdy. 1972. Streamflow Simulation, 2. The Broken Line Process as a Potential Model for Hydrologic Simulation, Water Resour. Res., 8(4), 931-941.

Mil'shtein, G.N. 1974. Approximate Integration od Stochastic Differential Equations, Theory of Prob. and Its Applic., 19, 557-562.

Morel-Seytoux, H.J. 1976. Derivation of Equations for Rainfal1 Infiltration, Journal of Hydrology, 31, pp. 203-219.

Morel-Seytoux, H.J. 1980. Methodology for Prediction of Variable Rainfall Infiltration, HYDROWAR Program, Engineering Research Center, Colorado State University, Fort Collins, Colorado, 9p. 
Morel-Seytoux, H.J. and J. Khanji. 1974. Derivation of an Equation of Infiltration, Water Resour. Res., 10(4), 795-800.

Nash, J.E. 1957. The Form of the Instantaneous Unit Hydrograph, Intern. Assoc. Scientif. Hydrol., Public. 45, 114-121.

Nash, J.E. 1960. A Unit Hydrograph Study with Particular Reference to British Catchments, Proc. Inst. Civ. Eng., 17, 249-282.

0'Connel1, P.E. 1974. Stochastic Modelling of Long-term Persistence in Streamflow Sequences, Ph.D. Thesis, Imperial Coll., Univ. of London, 284p. (Original not seen; cited in DeCoursey, 1982).

0'Conne11, P.E. 1977. ARIMA Processes in Synthetic Hydrology. In: Mathematical Models for Surface Hydrology, ed. by T.A. Ciriani, U. Maione, and J.P. Wallis, John Wiley and Sons, 51-70.

0'Connor, K.M. 1976. A Discrete Linear Cascade Model for Hydrology, Jour. of Hydrol., 29, 203-242.

Parzen, E. 1962. Stochastic Processes, Holden-Day, 324 p.

Philip, J.R. 1969). Theory of Infiltration, in: Advances in Hydroscience, Vol. 5, V.T. Chow, Ed., pp. 216-291.

Quimpo, R.G. 1973. Link Between Stochastic and Parametric Hydrology, Jour. Hydraul. Div., Proc., ASCE, 99(HY3), 461-470.

Rao, N.J., J.D. Borwankar and D. Ramkrishna. 1974. Approximate Integration of Stochastic Differential Equations, SIAM Jour. of Control, 12(1), 124-139

Rodriguez-Iturbe, I. and J.B. Valdes. 1979. The Geomorphologic Structure of Hydrologic Response, Water Resour. Res., 15(6), $1409-1420$.

Rosso, R. 1984. Nash Model Relation to Horton Order Ratios, Water Resour. Res., 20(7), 914-920.

Russo, D. and E. Bresler. 1981. So il Hydraulic Properties as Stochastic Processes, 1. An Analysis of Field Spatial Variability, Soil Sci. Amer. Jour., 45, 682-687.

Salas, J.D., J.W. Delleur, V. Yevjevich, and W. Lane. 1980. Applied Modeling of Hydrologic Time Series, Water Resour. Public, 484 p.

Salas, J.D. and R.A. Smith. 1981. Physical Basis of Stochastic Models of Annual Flows, Water Resour. Res., 17(2), 428-430.

Skaggs, R.W. 1982. Infiltration, Chapter 4 in: Hydrologic Modeling of Small Watersheds, Ed. by C.T. Haan, H.P. Johnson and

D.t. Brakensiek, Amer. Soc. of Agric. Engineers, 119-166. 
Smith, R.E. and H.A. Schreiber. 1974. Point Processes of Seasonal Thunderstorm Rainfal1, 2. Rainfall Depth Probabilities, Water Resour. Res., 10(3), 418-423.

Snyder, D.L. 1975. Random Point Processes, John Wiley and Sons.

Soong, T.T. 1973. Random Differential Equations in Science and Engineering, Academic Press, New York.

Stratonovich, R.L. 1966. A New Representation for Stochastic Integrals and Equations, SIAM Jour. of Control, 4, 362-371.

Thomas, H.A. and M.B. Fiering. 1962. Mathematical Synthesis of Streamflow Sequences for the Analysis of River Basins by Simulation, in: Design of Water Resources Systems by A. Mass, M.M. Hufschmidt, R.Dorfman, H.A. Thomas, S.A. Margl in and G.M. Fair, Haryard Univ. Press, Cambridge, Massachussetts (Original not seen; cited in Koch, 1982).

Todorovic, P. and V. Yevjevich. 1969. Stochastic Processes of Precipitation, Hydrology Paper No. 35, Colorado State University, Fort Collins, Colorado, 61p.

Todorovic, P. and D.A. Woolhiser. 1975. A Stochastic Model of n-Day Precipitation, Jour. Appl. Meteor., 14(1), 17-24.

Unny, T.E. 1984. Numerical Integration of Stochastic Differential Equations in Catchment Modeling, Water Resour. Res., 20(3), $360-368$.

Valencia, R.D. and J.C. Schaake, Jr. 1973. Disaggregation Processes in Stochastic Hydrology, Water Resour. Res., 9(3), 580-585.

Weiss, G. 1973. Shot iloise Model for Synthetic Generation of Multisite Streamflow Data, Symp. UNESCO, WMO, Intern. Assoc. Hydrol. Sci., Madrid.

Wood, E.F. 1976. An Analysis of the Effects of Parameter Uncertainty in Deterministic Hydrologic Models, Water Resour. Res., 12(5), 925-932.

Wright, D.J. 1974. The Digital Simulation of Stochastic Differential Equations, IEEE Trans. on Automatic Control, 75-76

Wu, I.P. 1963. Design Hydrographs for Small Watersheds in Indiana, Jour. Hyraul. Div., Proc., ASCE, 89(HY6), 35-66.

Yaron, D., G. Strateener, D. Shimshi and M. Weisbrod. 1973. Wheat Response to Soil Moisture and Optimal Irrigation Policy Under Conditions of Unstable Rainfall, Water Resources Research, 9(5), pp. 1145-1153. 
Yevjevich, V. 1963. Fluctuations of Wet and Dry Years, Part I: Research Data Assembly and Mathematical Models, Hydrol. Pap. 1, Colorado State Iniv., Fort Collins, Colorado. 